SLAC-PUB-2100

March 1978

$(\mathrm{T} / \mathrm{E})$

\title{
CHARM AND BEYOND
}

\author{
Thomas Appelquist*t \\ J. Willard Gibbs Laboratory, Yale University \\ New Haven, Connecticut 06520 \\ R. Michael Barnett** \\ Stanford Linear Accelerator Center, Stanford University \\ Stanford, California 94305 \\ Kenneth Lanetrits \\ Lyman Laboratory of Physics, Harvard University \\ Cambridge, Massachusetts 02138
}

To be published in

Annual Review of Nuclear and Particle Science

Vol. 28

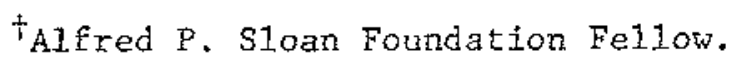

*Research supported in part by the Department of Energy under contract no. EY-76-C-02-3075.

**Research supported in part by the Department of Energy.

***Research supported in part by the National Science Foundation under contract no. PHY77-22864. 


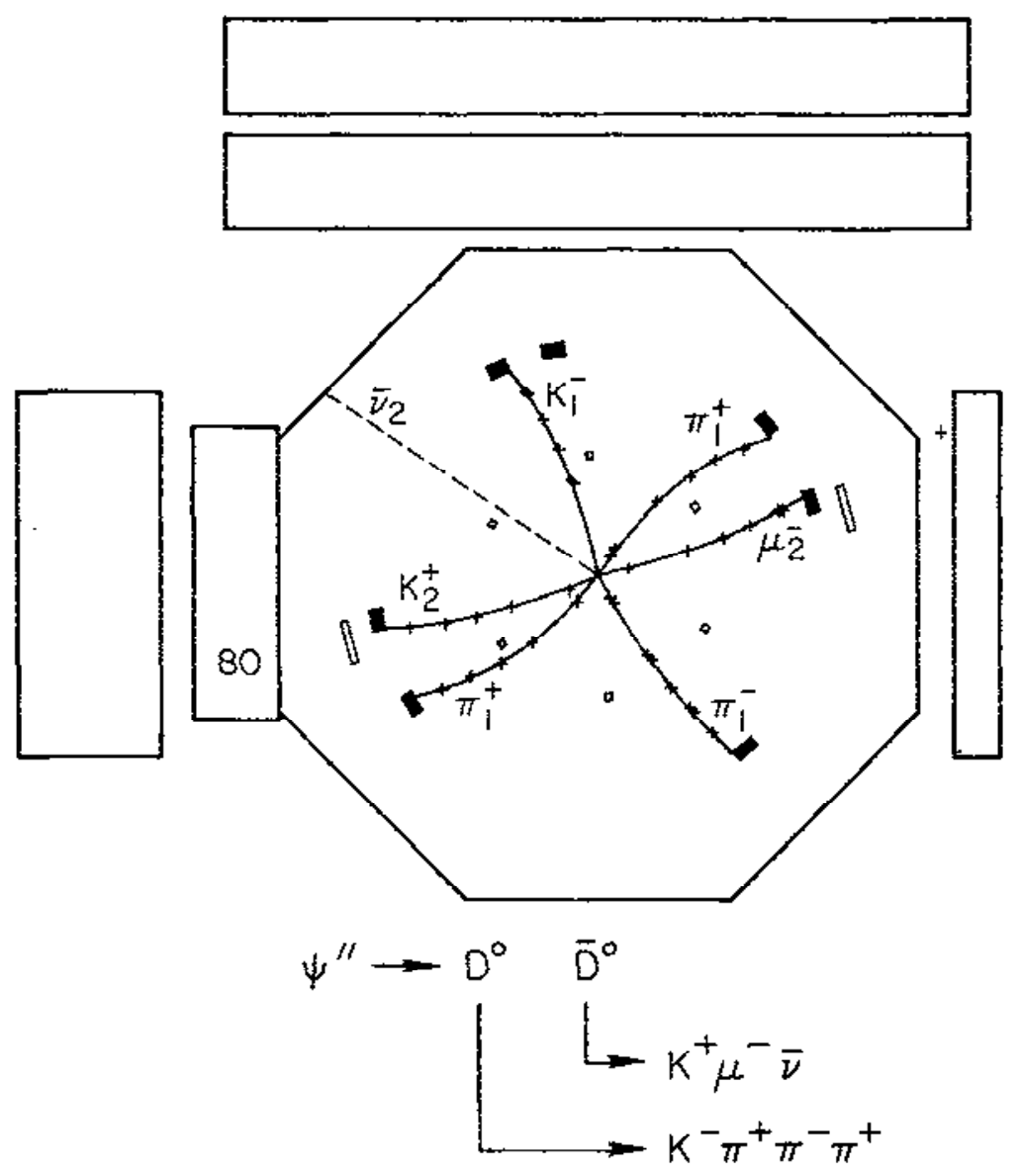

as seen by the SLAC-LBL Mark I detector 


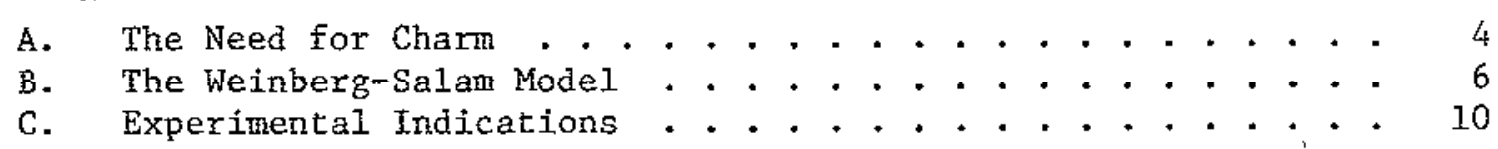

II QUANTUM CHROMODYNAMICS . . . . . . . . . . . . . . . . 13

A. Background. . . . . . . . . . . . . . . . . 13

B. The Hidden Color Hypothesis . . . . . . . . . . . . 13

C. The Color SU(3) Gauge Theory ................ 14

D. Properties of Quantum Chromodynamics. . . . . . . . . 19

III CHARMONIUM AND BEYOND . . . . . . . . . . . . . . . . . . . 24

A. An Experimental Overview ............... 26

B. Foundations of the Chamonium Model . . . . . . . . . 30

C. The Basic Charmonium Model ............... 34

D. Including Spin Dependence . . . . . . . . . . . . . 50

E. Coupling Charmonium to Its Decay Channels . . . . . . . . 57

F. Beyond Charmonium ................ 70

IV CHARMED HADRONS ........................ 78

A. Theory .................... . . 78

B. Experiment ................. . 86

V BEYOND CHARM . . . . . . . . . . . . . . . . . . . . . 94

A. Motivations .................... . . 9 94

B. Extending the Standard Model. . . . . . . . . . . 98

VI PRODUCTION BY NEUTRINOS, HADRONS AND PHOTONS . . . . . . . . 103

A. Production by Neistrinos ................ 103

B. Hadroproduction of Heavy Particles . . . . . . . . . . . 109

c. Photoproduction of $\psi$ and Charm ........... 116

VII NEUTRAI CURRENT INTERACTIONS . . . . . . . . . . . . . . . 119

VIII CONSERVATION LAWS . . . . . . . . . . . . . . . . . . 124

A. CP Violation .................... . . 124

B. Mun-Number Nonconservation .............. . 127

IX ATTEMPTS AT A GRAND SYNTHESIS . . . . . . . . . . . . . 132 


\section{INTRODUCTION}

The physics of elementary particles has changed dramatically during the 1970's, especially during the last three or four years. A new quark carrying a new quantum number called charm has been discovered and there is mounting evidence for the existence of a yet heavier quark. A great deal has been learned about the structure of the weak interactions, and there is considerable optimism that we are beginning to understand strong interaction dynamics at a fundamental level. Indeed, some visionaries are already attempting grand syntheses of the strong, weak and electromagnetic interactions. In this paper, we will describe these theoretical and experimental developments, emphasizing the role of the new heavy quarks. It is largely a review for nonspecialists but specialists will find some new results or at least some new perspectives.

Hadrons, that is mesons and baryons, are made of quarks (1); after the events of the last three years there are no longer any skeptics. Since many of the details of the quark model of hadrons are discussed by 0 . W. Greenberg in a paper appearing in this issue of the Annual Review (2), we only recall some of the basic properties of the old quarks to prepare for our discussion of the new ones. Until 1974, all the known mesons and baryons could be understood as quark-antiquark and three quark bound states respectively where the quarks come in three varieties or "flavors," commoniy called $u, d$ and $s$. The $u$ and $d$ (up and down) quarks form an lsotopic spin sU(2) doublet and are the constituents of the nucleons and mesons of nuclear physics. The heavier $s$ (strange) quark can bind with the others or with itself to produce the socalled strange particles that fill out the SU(3) multiplets of Gell-Mann (I). The relative heaviness of the strange quark means, of course, that this SU(3) symmetry is rather badly broken. All of these quarks have spin $1 / 2$ and carry 
fractional electric charge. The u quark has charge $2 / 3$ and the $d$ and $s$ quarks have charge $-1 / 3$.

\section{A. THE NEED FOR CHARM}

With the success of $S U(3)$ in the early $1960^{\prime}$ s, many people soon considered the possibility that yet heavier quarks might exist (3). They would presumably be constituents of hadrons too heavy and too short lived to have been seen at the time. It was partly a matter of "why not?" and partly motivated by primitive notions of quark-1epton symetry. There were four leptuns -- the electron, the muon and their respective neutrinos--so why shouldn't there be four quarks? Quark-lepton synmetry continues to be an important guiding principle in attempting to unify the fundamental forces of nature, but it seems likely that it will ultimately take a more subtle form than equal numbers of each.

It was a problem with weak interaction phenomenology that led Glashow, Iliopoulos and Maiani (GIM), in their classic paper of 1970 , to provide a genuine raison d'etre for a fourth quark (4). It had been known for decades that the weak interactions, such as neutron $\beta$-decay and $\mu^{-} \rightarrow \mathrm{e}^{-}+\bar{\nu}+\mathrm{e}^{+}{ }_{\mu}$, could all be described by the interaction of two charge-changing currents with an interaction strength $G_{F} \approx 10^{-5} / \mathrm{M}_{\mathrm{p}}^{2}$. It is now known that neutrai currents also play a role in the weak interactions. Processes like $\nu_{\mu}+p \rightarrow \nu_{\mu}+p$ have been observed and require both vector and axial vector neutral current interactions with strength of order $G_{F}$. In 1970, the neutral weak currents had not been seen experimentally but, for the most part, neither had they been ruled out at the level $G_{F}$. It was expected by some people that they would appear at this level and this expectation was given a sound basis with the proof, a year later, of the renormalizability of gauge theories of the weak 
and electromagnetic interactions. More about that shortly.

There was one embarrassing problem with this state of affairs. One class of neutral current interactions, those involving strangeness-changing hadronic weak currents, was known to be tremendously suppressed. The branching ratios (5)

$$
\frac{\Gamma\left(K_{L}^{0}+\mu^{+} \mu^{-}\right)}{\Gamma\left(K_{L}^{0} \rightarrow a 11\right)} \sim 10^{-8} \quad \frac{\Gamma\left(K^{ \pm}+\pi^{ \pm} e^{+} e^{-}\right)}{\Gamma\left(K^{ \pm}+a 11\right)}=(2.6 \pm 0.5) \times 10^{-7}
$$

exhibit the problem. That a strangeness-changing neutral current; coupling with strength $G_{F}$, might have been expected can be seen by examining the structure of the charge-changing hadronic weak current

$$
\begin{aligned}
J_{ \pm}^{\mu} & =\bar{q} \gamma^{\mu} \frac{1}{2}\left(1-\gamma_{5}\right) T_{ \pm} q \\
& \equiv \bar{q}_{L} \gamma^{\mu} T_{ \pm} q_{L} .
\end{aligned}
$$

The quark spinor $q$ contains $4 \times N$ components where $N$ is the number of flavors and $T_{ \pm}$is an $N \times N$ matrix which changes the electric charge by \pm 1 unit. $\mathrm{q}_{\mathrm{L}} \equiv \frac{1}{2}\left(1-\gamma_{5}\right) \mathrm{q}$ is the left-handed part of the quark field ${ }^{1}$. It was expected by some, and unified theories demanded, that there should exist a neutral partner of these two currents

$$
\mathrm{J}_{0}^{\mu}=\bar{q} \gamma^{\mu} \frac{1}{2}\left(1-\gamma_{5}\right) \mathrm{T}_{0} \mathrm{q}
$$

coupling with the same strength $G_{F}$, where

$$
T_{0}=\left[T_{+}, T_{-}\right]
$$

The charged current was known to have the Cabibbo form (6)

$$
J_{+}^{\mu k}=\bar{u} \gamma^{\mu \mu} \frac{1}{2}\left(1-\gamma_{5}\right)\left(\mathrm{d} \cos \theta_{c}+s \sin \theta_{c}\right)
$$


where $\sin \theta_{c}=0.23$. Therefore, the neutral current must contain a piece of the form

$$
J_{0}^{\mu}=\bar{s} \gamma^{\mu} \frac{1}{2}\left(1-\gamma_{5}\right) d \sin \theta_{c} \cos \theta_{c}+\ldots
$$

which gives rise to $\Delta S=1$ neutral interactions of order $G_{F}$.

The GIM solution (4) was to introduce a new, heavy, charge $2 / 3$ quark $c$ with a left-handed weak coupling to the orthogonal Cabibbo combination $s \cos \theta_{c}-d \sin \theta_{c}$. The $c$ quark was postulated to carry a new quantum number charm, conserved by the strong interactions. It can then easily be checked that with

$$
J_{+}^{\mu}=\vec{u} \gamma^{\mu} \frac{I}{2}\left(1-\gamma_{5}\right)\left(d \cos \theta_{c}+s \sin \theta_{c}\right)+\bar{c} \gamma^{\mu} \frac{1}{2}\left(1-\gamma_{5}\right)\left(s \cos \theta_{c}-d \sin \theta_{c}\right),
$$

the $\Delta S=1$ piece of $J_{0}^{\mu} E q .(1.6)$ is cancelled and the neutral current is, in fact, diagonal in all flavors. This current couples to itself and to leptonic neutral currents with strength $G_{F}$ and, to lowest order in this interaction, the reactions Eq. (1.1) are forbidden. Higher order corrections might, of course, induce such reactions and because the branching ratios (1.1) are so small, this must be looked at carefully. It is only possible to do this in a renormalizabie theory so we turn next to a discussion of gauge theories of weak and electromagnetic interactions.

B. THE WEINBERG - SALAM MODEL

Unified gauge theories provide the general framework for most modern work on weak and electromagnetic interactions. The prototype for all such models is based on the group $\operatorname{SU}(2) \times U(1)$ and was first written down in detail by Weinberg in 1967 (7). It remains viable today in the face of a large amount of experimental data and will survive as at least a sub- 
group of the ultimate weak and electromagnetic theory.

Unified gauge theories are constructed by generaljzing what is known about electromagnetic interactions to include the weak interactions. The inclusion of strong interactions in this framework will be described in the next section. The forces are all mediated by the exchange of spin one bosons corresponding to vector gauge fields which are present to maintain the local gauge invariance of the Lagrangian. In electrodynamics, this means invariance under a space-time dependent phase transformation $\psi(x) \rightarrow e^{i Q \theta(x)} \psi(x)$ on each matter field of charge $Q$, along with the transformation $A_{\mu}(x) \rightarrow A_{\mu}(x)$ $+\partial_{\mu} \theta(x)$ on the electromagnetic field. Invariance is insured if $A_{\mu}$ enters the Lagrangian only in the gauge covariant derivative $D_{\mu} \psi=\left(\partial_{\mu}-\right.$ IQA $\left._{\mu}\right) \psi$ and if derivatives of $A_{\mu}$ enter only through the electronagnetic field tensor $\mathrm{F}_{\mu \nu}=\partial_{\mu} \mathrm{A}_{\nu}-\partial_{\nu} \mathrm{A}_{\mu}$. By using these ingredients in a minimal way (excluding nonrenormalizable couplings), one obtains the Maxwe1l-Dirac theory for photons interacting with charged spin $1 / 2$ particles of mass $m$

$$
\mathscr{L}=\bar{\psi}\left[i \gamma^{\mu} \mathrm{D}_{\mu}-\mathrm{m}\right] \psi-\frac{1}{2} \mathrm{~F}_{\mu \nu} \mathrm{E}^{\mu \nu}
$$

The phase transformations of electrodynamics form the group $U(1)$, the group of unitary $1 \times 1$ matrices. Since both neutral and charge changing currents play a role in the weak interactions, the corresponding group must be larger, but the gauge principle can be caxried over by associating a separate gauge field $A_{\mu}^{a}(x)$ with each infinitesimal parameter of the group (8). The minimal possibility is $S U(2)$ and, since this is a three parameter group, there will be three gauge fields forming an isotopic triplet. The Lagrangian will take the form of Eq. (1.8) except that now $F_{\mu v}$ will contain a tern quadratic in $A_{\mu}^{a}$ and a coupling matrix will muliply the charge $Q$ in $D_{\mu} \psi$. Such a theory can be proven to be renormalizable ${ }^{2}(9)$. 
The problem is that the weak bosons must be very massive--they have not yet been seen and the lower limit on the mass is about $25 \mathrm{GeV}$ (10). The addition

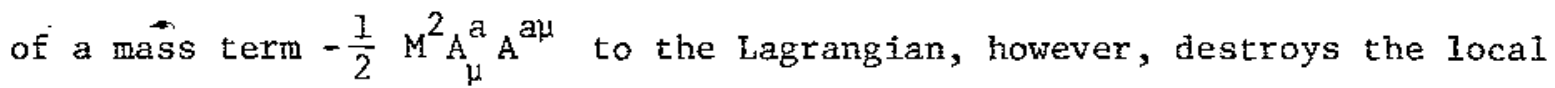
gauge invariance and the renormalizability. The solution is to preserve the local gauge invariance of the Lagrangian, but allow it to be spontaneously broken, that is, not respected by the physical states. There is a general theorem that whenever this happens, massless scalar particles, known as Goldstone bosons, must be present (11). However, in gauge theories these quanta get mixed in with the longitudinal parts of the gauge fields. They allow the gauge bosons to become massive by providing the zero helicity degree of freedom which is forbidden to a massless vector boson. This is called the Higgs mechanism (12). A particularly simple way of realizing this is to introduce into the Lagrangian a rultiplet of elementary spinless fields. After spontaneous symetry breaking, some of these become the Goldstone bosons absorbed by the gauge fields, while the remaining members of the multiplet survive as physical massive scalar particles known as Higgs bosons.

A unified model of this sort must incorporate both a weak gauge group and the electromagnetic gauge group. The SU(2) $\times$ U(1) model involves four gauge bosons. The spontaneous breakdown is arranged to preserve a local U(1) subgroup so that one boson stays massless and is identified with the photon, while the other three $\left(W^{+}\right.$and $Z^{\circ}$ ) become the intermediate bosons of the weak interactions. In the original form of the model (7), the left-handed leptons and quarks are put into SU(2) doublets and the right-handed components are in SU(2) singlets. The fermions are massless in the Lagrangian; their mass, along with the weak boson masses, arises from the spontaneous symmetry break- 
down. There are two independent coupling constants $g$ and $g^{\prime}$ for the SU(2) and $U(1)$ groups respectively. The electric charge is given by $e=g g^{\prime} /\left(g^{2}+g^{2}\right)^{\frac{1}{2}}$ and since $W^{ \pm}$exchange describes the familiar process of $B$-decay, the Fermi coupling constant $\mathrm{G}_{\mathrm{F}}$ is given by

$$
\frac{G_{F}}{\sqrt{2}}=\frac{g^{2}}{8 M_{W}^{2}}
$$

It follows that $M_{W}=\left(\frac{e^{2}}{\sqrt{32} G_{F}}\right)^{\frac{1}{2}} \frac{1}{\sin \theta_{W}}=37.3 \mathrm{GeV} / \sin \theta_{W}$, where $\theta_{W}$ is the Weinberg angle defined by $\tan \theta_{W}=g^{1} / g$. In the simplest version of the theory, there is one complex Su(2) doublet of Higgs fields, and the $z^{\circ}$ mass is given by $M_{Z}=M_{W} / \cos \theta_{W}$.

In this model, there remains one massive physical higgs scalar. Its mass is a free parameter theoretically, but its presence in the theory is absolutely crucial for renormalizability. It has by now been proven that such theories are, in fact, renormalizable to all orders (9). Spontaneously broken gauge theories of weak and electromagnetic interactions are theories in the same sense that quantum electrodynamics itself is.

Let us now return to the problem of $\Delta S=1$ neutral currents in the four quark GIM model. A process which is $O\left(G_{F}\right)$ in Born approximation will be $O\left(G_{F} e^{2}\right)$ at the one loop level in a renormalizable gauge theory. If a process is forbidden to lowest order, however, it is not hard to see that it will also vanish to $O\left(G_{F} e^{2}\right)$. Consider the decay $\mathrm{K}_{\mathrm{L}}^{\mathrm{O}} \rightarrow \mu^{+} \mu^{-}$for example. It can proceed through an intermediate state consisting of two charged bosons, but the graph with an exchanged $c$ quark is exactly cancelled by the graph with an exchanged u quark in the limit $\mathrm{m}_{\mathrm{u}}=\mathrm{m}_{\mathrm{c}} \cdot$. The amplitude is of order $G_{F}^{2}\left(m_{c}^{2}-m_{u}^{2}\right)$ as long as $m_{c}, m_{u}<<M_{W}$. This can be made consistent with 
rates and 1 imits such as those in $\mathrm{Eq}$. (1.1) with $\mathrm{m}_{\mathrm{c}} \approx 1-2 \mathrm{GeV}$. The mass difference between the $\mathrm{K}_{\mathrm{L}}^{0}$ and $\mathrm{K}_{\mathrm{S}}^{0}$ mesons arises from a similar interaction and led Gailiard and Lee (13) in 1973 to a similar estinate for $\mathrm{m}_{\mathrm{C}}$. These estimates are oniy approximate due to uncertainties associated with strong interaction corrections, but they later turned out to be qualitatively correct. C. EXPERIMENTAL INDICATIONS

The first direct experimental evidence for the existence of the charmed quark came from $\mathrm{e}^{+} \mathrm{e}^{-}$annihilation into hadrons. It was widely expected that $\mathrm{R}(\mathrm{W}) \equiv \sigma_{\text {tot }}\left(\mathrm{e}^{+} \mathrm{e}^{-} \rightarrow\right.$ hadrons $) / \sigma\left(\mathrm{e}^{+} \mathrm{e}^{-} \rightarrow \mu^{+} \mu^{-}\right)$would become constant above center of mass energy $W=1-2 \mathrm{GeV}$. The theoretical basis for this expectation and the value of the constant will be given in Chapter II. When this ratio was first measured above $W=3 \mathrm{GeV}$ at the Cambridge Electron Accelerator in $1970(14)$, it was found to be well above the expected value--and apparently rising with $W$ ! These measurements, eventually confirmed at SLAC (15) and DESY (16), gave hope to the small band of charm enthusiasts.

The next piece of evidence for charm came from the neutrino scattering experiments at Fermilab. In the collision of mon-type neutrinos with matter, events were discovered which contained a $\mu^{+} \mu^{-}$pair in the final state (17). These events, both in character and rate, could be naturally explained by charm excitation. The hadronic current Eq. (1.7) can couple to the muonic current $\bar{\mu} \gamma^{\mu} \frac{1}{2}\left(1-\gamma_{5}\right) \nu_{\mu}$ through $W^{+}$exchange leading to a muon and $c$ quark in the final state. The $c$ quark can decay by this same interaction into a lepton-neutrino pair and preferentially a strange quark. This source of dimuon pairs requires the presence of $K$ mesons in the final state in most events, and that was indeed verified somewhat later (17).

The case was clinched by the spectacular events of November 1974. The 
sinultaneous discovery of the $\psi / J$ resonance ${ }^{3}$ by groups at SLAC (18) and Brookhaven (19) and the subsequent measurement of excited states (20) and radiative transitions (21) could only be understood in terns of the existence of a new quark of mass around $1.5 \mathrm{GeV}$. In the $\psi$ it was bound to its own antiquark but, before long, particles made of one heavy and one or more old quarks were discovered (22), and their weak decay properties made it clear that the new quark was precisely the charmed quark predicted by GIM. The discovery of the Upsilon $T$ in July 1977 (23) will perhaps be the beginning of a similar story. At least two and perhaps three states in the mass range 9.5 to $10.5 \mathrm{GeV}$ have been discovered in the reaction $p+B e+T+\ldots$ $+\mu^{+} \mu^{-}+\ldots$ and it is irresistable to guess that they are the ground state and tho radial excitations of yet another quark and its antiquark. In the case of charmonium, it is the $\mathrm{e}^{+} \mathrm{e}^{-}$colliding beam machines that have produced the most important discoveries. Unfortunately, the highest energy presently available with these machines is about $8 \mathrm{GeV}$ which puts the upsilon just out of range. However, the next generation of higher energy colliding bean machines at SLAC, DESY, and Cornell will be turning on in the near future. They will, no doubt, produce a great deal of information about the upsilon and even heavier quark antiquark systems if they exist.

It is important to mention one more experimental development here. In 1975 , the SIAC-LBL group announced the discovery (24) of a new particle $\tau$ with $m_{\tau} \approx 1.8 \mathrm{GeV}$. The initial evidence came in the form of the "anomalous" events $e^{+} e^{-} \rightarrow e^{ \pm}+\mu^{+}+$ missing energy indicating a primary process $e^{+} e^{-}+\tau^{+} \tau^{-}$followed by weak decay of the $\tau^{+}$and $\tau^{-}$. With further study, it now seems clear that the $\tau$ is a lepton (25). These experiments and the $\tau$ properties will not be discussed in detail, but it is important to keep its existence in mind throughout. On the theoretical side, it must loom large in any attempts at grand synthesis or 
considerations of quark-lepton symetry. Experimentally, there is the curious fact that its mass is so close to that of the charmed quark. In $e^{+} e^{-}$annihilation, charm threshold and $\tau^{+} \tau^{-}$threshold come nearly on top of each other, so that the experimental study of each is complicated by the other. 


\section{QUANTUM CHROMODYNAMICS}

\section{A. BACKGROUND}

Even though charm was invented in response to problems with the weak and electromagnetic interactions, its discovery has had a considerable impact on strong interaction physics. The strong interactions have been a notoriously difficult problem for several decades and one night ask what impact the discovery of new strongly interacting quarks could have on our understanding of these forces. The answer is that early in 1973, a little more than a year before the experimental discovery of the $\psi / J$, a remarkable theoretical discovery was made (26) which has led to the growing consensus among theorists that we may finally have in hand a fundamental theory of the strong interactions. This has not yet been proven, but the measured properties of heavy quarks have reinforced this view and if yet heavier quarks are discovered, the reinforcement should become even stronger. The interplay of charm and this candidate theory of strong interactions called, quantum chromodynamics (QCD), will be discussed throughout the paper.

B. THE HIDDEN COLOR HYPOTHESIS

The first ingredient in QCD is a new, completely hidden, quantum number known as color (27). This notion is explained in some detail in the accompanying article by Greenberg (2) and so we shall only sumarize the essentials. Several problems with quark phenomenology have suggested that the number of quarks should be tripled so that each type of quark $u, d, s, c, \ldots$ comes in three so-called colors. In the most popular version of the color model, all the fundamental forces respect the symmetry under color interchange (28). This symmetry can be viewed as a new SU(3) symmetry and the observed hadrons are all singlets with respect to this new SU(3). 
There are several reasons for believing in the color hypothesis. With color, the ground states of baryons can be simply understood in the quark model without abandoning Fermi-Dirac statistics. Consider the $\Omega^{-}$for example; it consists of three $s$ quarks in an orbital $S$ state with spins aligned, leading to an overall symmetric wave function. With three colors, however, overall antisymmetry can be restored if the $\Omega^{-}$is a color singlet. Color also plays a sort of counting role, bringing up the value of certain amplitudes to agree with experiment. One example is the decay $\pi^{\circ} \rightarrow \gamma \gamma(29)$, and another is the value of $\sigma_{\text {tot }}\left(\mathrm{e}^{+} \mathrm{e}^{-} \rightarrow\right.$ hadrons) within the free quark (parton) approximation (30). There, neglecting fermion masses,

$$
R(W) \equiv \frac{\sigma_{t o t}\left(e^{+} e^{-} \rightarrow \text { hadrons }\right)}{\sigma\left(e^{+} e^{-} \rightarrow \mu^{+} \mu^{-}\right)}=\sum_{i} Q_{i}^{2}
$$

where $Q_{i}$ is the electric charge of a quark of type $i$ in units of e. The inclusion of color in this sum raises the prediction from $2 / 3$ to 2 below charm threshold. This is a good first approximation to the experimental value of $\mathrm{R}(31-34)$ and within $\mathrm{QCD}$ it also turns out to be a good first approximation theoretically.

\section{THE COLOR SU(3) GAUGE THEORY}

QCD is constructed by extending the global SU ${ }_{c}(3)$ color symmetry to a local gauge symetry. This requires the introduction of massless vector gauge fields $A_{\mu}^{a}(x), a=1,2, \ldots 8$, transforming according to the adjoint representation of $\mathrm{SU}_{\mathrm{c}}(3)$. The eight galige fields are called colored gluons. The Lagrangian density is

$$
\mathscr{L}(\mathrm{x})=\overrightarrow{\mathrm{q}}(\mathrm{x})\left[i \gamma^{\mu} \mathrm{D}_{\mu}-\mathrm{M}_{\mathrm{o}}\right] \mathrm{q}(\mathrm{x})-\frac{1}{4} \mathrm{~F}_{\mu \nu}^{\mathrm{a}}(\mathrm{x}) \mathrm{F}^{\mathrm{a} \mu \nu}(\mathrm{x})
$$

where the quark field $q(x)$ contains $4 \times 3 \times N$ components, corresponding to the three colors and the unknown number $N$ of quark $f$ lavors. $M_{0}$ is the bare 
mass matrix, a product of Dirac and color untt matrices and a diagonal flavor matrix $\mathrm{M}_{0}^{\mathrm{AB}}$. The gauge field covariants are

$$
\begin{aligned}
& D_{\mu}=\partial_{\mu}-i g T^{a} A_{\mu}^{a} \\
& F_{\mu \nu}^{a}=\partial_{\mu} A_{\nu}^{a}-\partial_{\nu} A_{\mu}^{a}+g f^{a b c} A_{\mu}^{b} A_{\nu}^{c}
\end{aligned}
$$

and we normalize the $T$ matrices in the conventional way

$$
\mathrm{T}_{\mathrm{a}}=\frac{1}{2} \lambda_{\mathrm{a}}, \quad\left[\lambda_{\mathrm{a}}, \lambda_{\mathrm{b}}\right]=2 \text { if } \mathrm{abc}_{\mathrm{c}}, \quad \operatorname{Tr} \lambda_{\mathrm{a}} \lambda_{\mathrm{b}}=2 \delta_{\mathrm{ab}}
$$

The theory is invariant under the gauge transformation

$$
A_{\mu}(x) \equiv A_{\mu}^{a}(x) T^{a} \rightarrow U(x) A_{\mu}(x) U^{-1}(x)+\frac{i}{g} U(x) a_{\mu} U^{-1}(x) \quad q(x) \rightarrow U(x) q(x)
$$

where $U(x)=\exp \left\{i g \theta^{a}(x) T^{a}\right\}$.

While QCD bears a superficial resemblance to quantum electrodynamics, it is, in fact, considerably more complicated. It is a renormalizable theory, Iike QED, so that the ultraviolet divergences that appear in perturbation theory can be handled in the traditional way. Field theory Green's functions can be computed to any order in a power series expansion in a renormalized coupling constant. One expects this to be useful only if the coupling constant is small, however, and that depends on how the theory is renormalized.

It is here that QCD and QED part company.

In quantum electrodynamics, the renormalized coupling constant $\alpha$ can be defined at zero monentum transfer (infinite distances) since the nonzero electron mass prevents infrared divergences in the photon propagator in this 1imit. The potential energy between two charged particles separated by a distance $R>>1 / m e$ is $\alpha / R$, with $\alpha$ determined experimentally to be $1 / 137$. As a consequence of the $1 / R$ behavior, the asymptotic states of the theory are 
the charged particles and photons corresponding to the fields in the Lagrangian. At distances $R \ll 1 / \mathrm{m}_{\mathrm{e}}$, the charged shielding effect of vacuum polarization begins to disappear and the effective coupling strength begins to increase. The familiar one-loop result is

$$
V(R)=\frac{\alpha}{R}\left\{1+\frac{\alpha}{3 \pi} \log \left(R m_{e}\right)^{-1}\right\}
$$

and at distances $R \simeq \mathbb{m}^{-1} e^{-3 \pi / \alpha}$, the increase becomes substantial. Since the perturbation expansion breaks down at this point, the asymptotic behavior of QED as $R \rightarrow O$ is unknown. Fortunately, physics at laboratory distance scales is insensitive to this asymptotic ignorance.

QCD differs from QED in many ways, in particular in the way the effective coupling strength as computed in perturbation theory varies with distance. This different behavior is the important feature of QCD called asymptotic freedom (26). We shall describe this property with an eye toward our later discussion of its role in heavy quark physics. At the end of this chapter, some of the other known and conjectured properties of QCD will be summarized.

The Feynman rules for QCD can be generated using either functional methods or the canonical Hamiltonian formalism (35). In either case, a gauge must be chosen as in QED. In a general class of covariant gauges, the gluon propagator is

$$
D_{\mu \nu}^{a b}(k)=\delta^{a b}\left(g_{\mu \nu}-\xi \frac{k_{\mu} k v}{k^{2}}\right) \frac{1}{k^{2}+i \varepsilon}
$$

where $\xi$ is an arbitrary parameter specifying the gauge. The remaining Feynman rules, consisting of the quark propagator and the various vertices, can be read off directly from the Lagrangian (35). The only exception to this is that a careful treatment of unitarity demands the inclusion of fictitious scalar particlescalled Fadde'ev-Popov ghosts which propagate only 
around closed loops (36).

For some purposes, it is convenient to adopt the physical Coulomb gauge (35). The propagator then consists of an instantaneous potential part and a transverse gluon part

$$
\begin{aligned}
D_{\mu \nu}^{a b}(k) & =\delta^{a b} i \vec{k}^{2} \quad \mu=\nu=0 \\
& =\delta^{a b} \frac{i}{k^{2}+i \varepsilon}\left(\delta_{i j}-\frac{k_{i} k}{\vec{k}^{2}}\right) \quad \mu=i, v=j .
\end{aligned}
$$

A Fadde'ev-Popov ghost must again be included, but it is instantaneous and it couples only to transverse gluons. In either covariant or Coulomb gauge, Ward identities can be established and renormalizability proven.

Now consider again the potential energy between two charge sources separated by a distance $R(37,38)$. The charge is now color and the sources can be taken to be a very heavy quark and antiquark. If they are in a quantum mechanical color singlet state, then the Born approximation to the potential is $-C_{F} \alpha_{S} / R$ where $T_{i k}^{a} T_{k j}^{a}=C_{F} \delta_{i j}$ and $a_{s}=g^{2} / 4 \pi$. For $\operatorname{SU}(\mathbb{N}) C_{F}=\left(\mathbb{N}^{2}-1\right) /$ 2N. The one loop corrections to the potential are most conveniently computed in Coulomb gauge and, if light quarks are neglected for the moment, the two relevant diagrams are shown in Fig. 1 (37). The necessary renormalization subtractions cannot be performed at infinite separation because the loops are infrared divergent at this point. Some other arbitrary point, say $\mathrm{R}=\mu^{-1}$, must be chosen, and this necessarily brings in a new dimensional parameter $\mu$. In QED, such a parameter could be introduced; in QCD, because of the self-coupling of the gauge field, it mast be introduced. The static potential through one loop is 


$$
V(R)=-C_{F} \frac{\alpha}{R}\left\{1+\frac{5}{6} \frac{\alpha}{\pi} C_{A} \ln (R H)^{-1}-\frac{16}{6} \frac{\alpha \mu}{\pi} C_{A} \ln (R \mu)^{-1}\right\}
$$

where $\alpha_{\mu}=\mathrm{g}_{\mu}^{2} / 4 \pi$ is the coupling strength corresponding to the scale $R=\mu^{-1}$ and $f_{\text {acd }} f_{b c d}=C_{A} \delta a b=N \delta_{a b}$ for $S U(N)$. The two logarithnic modification terms correspond to Figs. Ib and la respectively. The first is vacuum polarization of transverse gluon pairs, a charge shielding effect similar to electron-positron vacuum polarization in QED. As expected, this contribution tends to make the effective coupling strength increase as $R \rightarrow 0$. The other contribution, unique to Yang-Mills theories, is a self-energy of the Coulonb field. It comes with the opposite sign and is larger in magnitude than vacum polarization. The net result is that the effective coupling strength decreases as $\mathrm{R}$ decreases. This is the property called asymptotic freedom.

It is not difficult to understand the physical mechanism behind asymptotic freedon (37). We shall not describe it in detail except to say that the Coulomb field self-energy produces a collimation of the color electric field lines connecting the quark to the antiquark. The collimation becomes more pronounced as $R \rightarrow \infty$, increasing the energy stored in the field relative to the pure Coulonb potential. The decrease of the collination as $\mathrm{R}$ decreases is the explanation of asymptotic freedom.

The behavior of the effective coupling strength as $R \rightarrow 0$ can be described to all orders in perturbation theory by a consideration of its scaling 
properties ${ }^{4}(39)$, The potential can be written quite generally in the form

$$
V(R)=-C_{F} \frac{\alpha\left(R \mu, \alpha_{\mu}\right)}{R}
$$

where $\alpha\left(R \mu, \alpha_{\mu}\right) \equiv \alpha_{S}(R)$ is the effective coupling strength, given through one Ioop by Eq. (2.9). The potential must be independent of the choice of $\mu$ and the condition $\frac{d}{d \mu} \alpha(R)=0$ can be used to show that

$$
R \frac{\partial}{\partial R} \alpha_{S}(R)=\beta\left(\alpha_{S}(R)\right)
$$

where

$$
\left.B(x) \equiv R \frac{\partial}{\partial R} \alpha(R \mu, x)\right|_{R=\mu}-1
$$

From Eq. (2.9)

$$
B(x)=\frac{11}{2} \frac{x}{\pi}+O\left(x^{2}\right)
$$

and, therefore, to lowest order, Eq. (2.11) can easily be integrated. The result is

$$
\alpha_{S}(R)=\frac{\alpha_{\mu}}{1+\frac{11}{2} \frac{\alpha_{\mu}}{\pi} \ln (R \mu)^{-1}}
$$

which agrees with Eq.(2.9) to lowest order and which goes to zero as $R \rightarrow 0$. In the opposite limit, Eq. (2.14) shows that the theory becomes strongly coupled and the perturbation expansion breaks down. In this sense, QCD and QED are oppositely behaved.

\section{PROPERTIES OF QUANTUM CHROMODYNAMTCS}

1. COVARIANT FORMULATION For most purposes, it is best to work in a covariant gauge. The asymptotic form Eq. (2.14) of the ruming coupling constant is gauge invariant, but its Feyman diagrammatic breakdown is completely diffexent in a covariant gauge (26). As a result, the physical mechanism behind asymptotic freedom is not as transparent as in Coulomb gauge. 
The momentum space effective coupling constant $\alpha_{s}\left(-q^{2}\right)$ is defined in terms of both propagator and vertex functions at a Euclidean momentum $q^{2}=q_{0}^{2}-q^{2}<0$. If there are $f$ quark flavors for which $m<<$, then

$$
\alpha_{s}\left(-q^{2}\right)=\frac{\alpha_{\mu}}{1+\left(\frac{11}{4}-\frac{1}{6} f\right) \frac{\alpha}{\pi} \ln \left(-q^{2} / \mu^{2}\right)}
$$

It is convenient to re-express $\alpha_{s}\left(-q^{2}\right)$ in the form

$$
\alpha_{s}\left(-q^{2}\right)=\frac{\pi}{\left(\frac{11}{4}-\frac{1}{6} f\right) \text { \& }\left(-q^{2} / \Lambda^{2}\right)}
$$

where $\Lambda=\mu \exp \left[-\pi /(11 / 2-f / 3) \alpha_{\mu}\right]$. This explicit1y exhibits the fact that $\alpha_{s}\left(-q^{2}\right)$ depends only on one parameter $\left(\alpha_{\mu}\right.$ and $\mu$ are not independent). $\Lambda$ must be determined experimentally, and then $\alpha_{s}\left(-q^{2}\right)$ is completely specified.

2. APPLICATIONS OF ASYMPTOTIC FREEDOM It is only possible to directly confront asymptotic freedom with experiment in those few situations where the measured quantity is sensitive only to the short distance behavior of QCD. The most important example is deep-inelastic electroproduction, where approximate Bjorken scaling (40) at momentum transfers larger than $1-2 \mathrm{GeV}$ indicates that the theory is nearly free. In order to make this precise, that is, to truly isolate short distance behavior from the data, one must make use of the Wilson operator product expansion (41). An explanation of this formalism falls beyond the purview of this paper and we simply summarize the situation: Short distance physics can indeed by isolated and the experimental data suggests that $\alpha_{s}\left(-q^{2}\right)<1$ for $\sqrt{-q^{2}}>1-2 \mathrm{GeV}$. Analyses of electroproduction typically suggest a value of $\mathrm{A}$ around $500 \mathrm{MeV}$. This gives $\alpha_{s}\left(-q^{2}\right) \simeq 0.5$ at $\sqrt{-q^{2}} \simeq 2 \mathrm{GeV}$. 
Another important application of asymptotic freedom is the total cross section for $\mathrm{e}^{+} \mathrm{e}^{-} \rightarrow$ hadrons (42). Moments of the cross section are related by dispersion relations to hadronic vacuum polarization at spacelike $\mathrm{q}^{2}$. This is sensitive only to short distance behavior and is therefore computable in perturbation theory for $-q^{2}$ large enough. It has been conjectured that asymptotic freedom can be applied directiy to $\sigma_{\text {tot }}\left(\mathrm{e}^{+} \mathrm{e}^{-} \rightarrow\right.$ hadrons $)$ for $\mathrm{q}^{2}=\mathrm{w}^{2}>0$ as though the final particles are quarks and giuons. This is true, at best, away from important thresholds such as charm-anticharm, and there the prediction for $R(W) \mathrm{Eq}$. (2.1) is

$$
R(W)=\sum_{i} Q_{i}^{2}\left\{1+\frac{\alpha_{s}(W)}{\pi}\right\} \text {. }
$$

This result can be stated in a somewhat more solid form by averaging $R$ over an interval in $W(44)$. But where $R$ shows no rapid variation with $W$, this shouldn't be necessary and Eq. (2.17) can be used reliably. The total cross section data is still rather poor for these purposes (31-34). Above charm threshold, the errors are large and the presence of the heavy $\tau$ Iepton (24) complicates matters. Below charm threshold ( $1 \mathrm{GeV}<\mathrm{W}<3 \mathrm{GeV}$ ), the experimental value of $R$ is somewhat above the parton model value of $\sum_{i} Q_{i}^{2}=2$. About the best that can be said now is that the data are consistent with $\Lambda \simeq 500 \mathrm{MeV}$.

There are many other situations where asymptotic freedom plays at least some role, Some examples are high monentum transfer hadron-hadron scattering (45) and the $\Delta I=1 / 2$ rule in nonleptonic weak decays (46). However, the short distance and long distance behavior of QCD cannot be cleaxly separated in these problems and they are perhaps less important for testing asymptotic freedom. There is one other possible application of asymptotic 
freedor which is very important for heavy quark physics. The $\psi$ particle is very long-lived and it has been suggested that this can be understood as a consequence of asymptotic freedom (47). This possibility will be discussed in detail in Section III, but we should emphasize here that if the narrow width of the $\psi$ can be explained in this way, a value of $\alpha_{S}\left(M_{\psi}^{2}\right) \simeq 0.2$ is required. Whether the analysis of electroproduction allows such a small effective coupling is not yet clear.

3. BEYOND PERTURBATION THEORY A widespread hope is that the spectrum of QCD consists only of the color singlet hadrons observed in the laboratory. The underlying quarks and gluons would then not be among the asymptotic states, existing only as the constituents of hadrons. To demonstrate this and to compute the masses and other properties of hadrons starting from the QCD Lagxangian is an extraordinarily difficult and completely unsolved problem. A discussion of the many approaches to this problem is beyond the scope of this review and we shall only make a few comments to provide some perspective for the next sections.

None of these conjectured features of QCD can be seen in perturbation theory. As the distance scale $\mathrm{R}$ increases, the effective coupling Eq. (2.14) or Eq. (2.16) increases and perturbation theory is no longer directly useful. One might hope that general properties could still be extracted from the perturbation expansions or that the series could be summed to deal with large distance effects, but even this seems unlikely. On the one hand, it can be shown that to any finite order of perturbation theory, there is no indication of confinement (48). The self-coupling of the gauge field suggests an infrared divergence structure much worse than QED, and one speculation was that this structure might have something to do with confinement. However, 
it has been shown (48) that properly defined transition probabilities are infrared finite order by order in a renornalized coupling constant $\alpha_{\mu}$. The situation is not unlike QED .

As far as suming the expansion is concerned, there is every indication that the series is not convergent. In fact, it would appear that the series is not even Borel summable (49) so that the perturbation expansion cannot be used to define the theory except for weak coupling. QCD is known to contain essential singularities at $\alpha_{\mu}=0$ even at the classical 1evel. The most important examples of this at the present are the instantons and other Euclidean field configurations with nontrivial topological structure (50). The ultimate role of these features of QCD in confinement is not yet known but they surely point up the inadequacy of perturbation theory. Perhaps the most ambitious attack on confinement and hadron structure has been the strong coupling expansion for QCD pioneered by Wilson (51). A short distance cutoff in the form of a spatial or space-time lattice eliminates the weakly coupled sector of the theory. A linear confining potential $V(R)$ vaR between quarks appears naturally as a consequence of the fact that the color electric flux is quantized on the lattice (52). The problem of taking the lattice spacing to zero is, however, unsolved and this is an important ingredient in computing the properties of hadrons on the lattice. The linear potential suggested by the lattice strong-coupling expansion has been applied rather successfully to charmonium spectrum computations; an example of the interplay between charm and (in this case) suggested properties of QCD. 


\section{CHARMONIUM AND BEYOND}

The discovery of narrow resonances in November 1974 had been anticipated theoretically (47). If charm was real, then narrow $\bar{c} \bar{c}$ bound states should exist below the threshold for charm production and the name charmonium was suggested, in analogy to positronium.

At the time it was, in fact, thought that the resemblance to positronium might be more than just an analogy $(47,53)$. Asymptotic freedom says that at short distances (Iess than about $1 / 5$ fermi) the strong interactions should behave nearly like $\alpha_{s} / \mathrm{r}$ with $\alpha_{S}=g^{2} / 4 \pi<<1$ (but of course $>1 / 137$ ). If the $c$ and $\bar{c}$ were to spend most of their time within $1 / 5$ fermi of each other, then the similarity with positronium would becone almost complete. It became clear, very quickly after the initial discoveries, that things were not going to be so simple. The radius of charmonium has turned out to be more like 1 fermi than $1 / 5$ fermi and the binding potential has a structure completely unlike the Coulomb potential of electrodynamics. Nevertheless, the qualitative resemblance to positronium is undeniable and the name charmonium has caught on.

This section reviews in some detail the major areas of theoretical research into this charmonium system. After a brief survey of the experimental situation in Sec. III.A, we lay the groundwork for what we shall call the charmonium model (Sec. III.B). This is the atomic model of heavy (c) quarks bound in a static, confining potential which, together with asymptotic Ereedom applied to short-distance processes, describes the spectrum and decay widths of states in the cer-system. 
The simplest version of the model is discussed in Sec. III.C, with spectal emphasis on the choice of a phenomenological potential and the spectrum of states and transition probabilities resulting therefrom. At this naive level, the model already provides a fairly good description of the charmonium system with only two glaring exceptions--the even-charge-conjugation states at 2.83 and $3.45 \mathrm{GeV}$. The main attempts to go beyond the basic model, by incorporating effects of quark spin-dependence and of coupling c $\vec{c}$ states to charmed hadron decay channels, are reviewed critically in Secs. III.D and E. In both cases, we have tried to motivate the directions research has taken, evaluate the outcomes and, by stressing the shortcomings of existing, work, hopefully point the way for future improvenent. Finally, Sec. III.F sumarizes the straightforward applications of the potential model to bound systems of still heavier quarks. Here, the data is still too limited for critical evaluation of the theory, but we can look forward to rigorous experimental tests in just a year or two.

Lack of space prevents our discussing other charmonium research topics. Most notable are the attempts to compute charmonium properties without recourse to a potential model. These include Regge-trajectory analysis of the spectrum (54), a topological s-matrix approach to understanding the small hadronic widths of charmonium (55), and the use of dispersion relations to (a) derive sum rules between leptonic widths of charmonium levels and integrals over the charm contribution to $R$ and (b) estimate two-photon widths of appropriate states by using sum rules derived from $\gamma \gamma$ scattering (56). 


\section{A. EXPERIMENTAL OVERVIEW}

There are several excellent, up-to-date reviews $(57,58,59)$ on the production and decay characteristics of the charmonium states (58 and 59 reference expermental work not cited explicitly here). We content ourselves here with a brief survey of what has been seen, with special emphasis on production via $\mathrm{e}^{+} \mathrm{e}^{-}$an- $^{-}$ nihilation; for other production mechanisms of $\psi / J$, see Sec. VI. Table 1 sumarizes the known properties of charmonium. A level diagram ${ }^{5}$ is shown in Fig. 2 . Except for the simultaneous discovery of $\psi / J$ in $\mathrm{e}^{+} \mathrm{e}^{-}$annihilation at SLAC (18) and in proton-beryllium collisions at Brookhaven (19), all the charmonium levels-including narrow states below charm threshold $\left(\mathrm{W}_{\mathrm{C}}=2 \mathrm{M}_{\mathrm{D}^{\circ}}=\right.$ $3.727 \mathrm{GeV}$ ) and broad ones above-have been discovered at the SLAC and DESY $\mathrm{e}^{+} \mathrm{e}^{-}$storage rings (SPEAR and DORIS). The experimental situation is incredibly beautiful and simple: States with $\mathrm{J}^{\mathrm{PC}}=1^{--}$, the quantum numbers of the photon, are produced directly and copiously as resonances in $e^{+} e^{-}$collistons; narrow states lying below $\psi^{\prime}$ (3684) and having even charge conjugation are observed in radiative transitions, $\psi^{\prime} \rightarrow \gamma \times$ and $\psi \rightarrow \gamma X$. It should be added that there remain additional charmonium levels to be discovered. An important one is the $1^{1} \mathrm{P}_{1}$ state with $J^{\mathrm{PC}}=1^{+ \text {t- }}$, expected near $3.45 \mathrm{GeV}$. In $e^{+} e^{-}$annihilation, this could be observed (with difficulty) only in the reaction chain

$$
\begin{aligned}
& \mathrm{e}^{+} \mathrm{e}^{-} \rightarrow \psi^{\prime}\left(3684 ; 2^{3} \mathrm{~s}_{1}\right)
\end{aligned}
$$

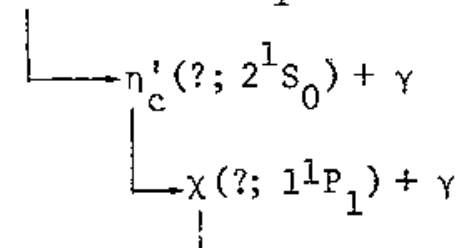

$$
\begin{aligned}
& \text { hadrons . }
\end{aligned}
$$


Table 1

The properties of charmonium. All data is taken from Ref. 59 which cites original work. Question marks indicate unknown values.

\begin{tabular}{|c|c|c|c|c|c|}
\hline Particle & $I^{G}\left(J^{P C}\right)$ & $\begin{array}{l}\text { Mass } \\
(\mathrm{MeV})\end{array}$ & $\begin{array}{c}\text { Full width } \\
\text { (MeV) }\end{array}$ & Decay Mode & Fraction (\%) \\
\hline$X(2830)$ & $?^{?}\left(?^{?+}\right)$ & $2830 \pm 30$ & & $Y \gamma$ & $>0.7$ \\
\hline$\psi / J(3095)$ & $0^{-}\left(1^{--}\right)$ & $3098 \pm 3$ & $0.069 \pm 0.015$ & $\begin{array}{c}e^{+} e^{-} \\
\mu^{+} \mu^{-} \\
\text {Direct hadrons } \\
\gamma \times(2830)\end{array}$ & $\begin{array}{c}7.3 \pm 0.5 \\
7.3 \pm 0.5 \\
86 \pm 2 \\
<1.7\end{array}$ \\
\hline$x(3415)$ & $0^{+}\left(0^{+1}\right)$ & $3413 \pm 5$ & $?$ & $\begin{array}{c}\gamma X(2830) \rightarrow 3 \gamma \\
\gamma X(2830) \rightarrow \gamma p \bar{p} \\
\gamma \psi(3095) \\
\text { Hadrons }\end{array}$ & $\begin{array}{c}0.013 \pm 0.004 \\
<0.004 \\
3 \pm 3\end{array}$ \\
\hline $\mathrm{P}_{\mathrm{c}} / \mathrm{x}(3510)$ & $0^{+}\left(1^{++}\right)$ & $3508 \pm 4$ & $?$ & $\begin{array}{l}\gamma \psi(3095) \\
\text { Hadrons }\end{array}$ & $35 \pm 16$ \\
\hline$x(3455)$ & $?^{?}\left(?^{?+}\right)$ & $3454 \pm 7$ & $?$ & $\gamma \psi(3095)$ & $>24 \pm 16$ \\
\hline$x(3550)$ & $0^{+}\left(2^{++}\right)$ & $3552 \pm 6$ & $?$ & $\begin{array}{l}\text { Y\&(3095) } \\
\text { Hadrons }\end{array}$ & $14 \pm 9$ \\
\hline$\psi^{\prime}(3684)$ & $0^{-}\left(1^{-\infty}\right)$ & $3684 \pm 4$ & $0.228 \pm 0.056$ & $\begin{array}{c}e^{+} e^{--} \\
\mu^{+}{ }^{+} \\
\psi \pi^{+} \\
\psi \pi^{\circ} \\
\psi \pi^{\circ} \\
\gamma \times(3415) \\
\gamma \times(3510) \\
\gamma \times(3550) \\
\gamma \times(3455) \\
\gamma \times(3455) \rightarrow \gamma \gamma \psi \\
\gamma \times(2830) \\
\text { Direct hadrons }\end{array}$ & $\begin{aligned} 0.88 & \pm 0.13 \\
0.88 & \pm 0.13 \\
33.1 & \pm 2.6 \\
15.9 & \pm 2.8 \\
4.1 & \pm 0.7 \\
7.3 & \pm 1.7 \\
7.1 & \pm 1.9 \\
7.0 & \pm 2.0 \\
& <2.5 \\
0.6 & \pm 0.4 \\
& <1.0 \\
& \approx 9\end{aligned}$ \\
\hline$\psi "(3772)$ & $0^{-}\left(1^{--}\right)$ & $3772 \pm 6$ & $28 \pm 5$ & $\begin{array}{l}e^{+} e^{-} \\
D^{0} D^{\circ} \\
D^{+} D^{-}\end{array}$ & $\begin{array}{l}56 \pm 3 \\
44 \pm 3\end{array}$ \\
\hline$\psi(4028)$ & $?\left(1^{-\infty}\right)$ & $4028 \pm 20$ & $\approx 50$ & $\begin{array}{c}e^{+} e^{-} \\
\text {Charmed mesons }\end{array}$ & $\begin{array}{l}\approx 0.002 \\
\approx 100\end{array}$ \\
\hline$\psi(4414)$ & $?\left(1^{--}\right)$ & $4414 \pm 7$ & $33 \pm 10$ & $\frac{\mathrm{e}^{+} \mathrm{e}^{-}}{\text {Charmed mesons }}$ & $\begin{array}{c}0.0013 \pm 0.0003 \\
\sim 100\end{array}$ \\
\hline
\end{tabular}


The nost striking feature of states lying below $\mathrm{w}_{c}$ is their very small total widths. In particular, first and second order electromagnetic decays such as $\psi^{\prime} \rightarrow Y X$ and $\psi_{\rightarrow} \mathrm{e}^{+} \mathrm{e}^{-}$are competitive with strong-interaction decays such as $\psi^{\prime} \rightarrow \psi \pi \pi$ and $\psi \rightarrow$ hadrons. If we assume that these high-mass mesons are ec bound states, it follows that decay to ordinary hadrons, not containing charmed quarks, must proceed by mutual annihilation of the $c \bar{c}$ pair. The reluctance of any $q \bar{q}$ pair within a single hadron to annihilate, known as the Okubo-Zweig-Iizuka (OZI) rule (60), has been observed in the light hadrons for a long time. The most notable example is the suppressed decay $\phi(s \vec{s}) \rightarrow$ $\pi^{+} \pi^{-} \pi^{\circ}$ (all containing u, d quarks). This empirical rule could be a simple consequence of asymptotic freedom in the case of heavy quarks.

Two features of $\mathrm{e}^{+} \mathrm{e}^{-}$annihilation make it ideal for discovery and study of the myriad of charmonium levels: First, the energy in a single beam, $E_{b}=\frac{1}{2} \mathrm{~W}$ ( $W=$ total center-of-mass energy), is known very precisely, to within $1-2 \mathrm{MeV}$. Second, the $\mathrm{J}^{\mathrm{PC}}=\mathrm{I}^{--}$states are produced at rest in the center of mass. Together, these allow very precise measurement of the mass of the directly produced states. The even-C states below $\psi^{\prime}$ are produced by "sitting on" $\psi^{\prime}$ or $\psi$, i.e., setting $E_{b}=\frac{1}{2} M_{\psi}$ - or $\frac{1}{2} M_{\psi}$, and observing their radiative decays. For this, three methods have been used:

1) Measurement of the invariant mass of the charged hadrons in decays such as

$$
\psi^{\prime} \rightarrow \pi^{+} \pi^{-} \pi^{+}{ }^{-}+\text {missing neutrals, }
$$

corresponding, perhaps, to $\psi^{\prime} \rightarrow x+\gamma, x \rightarrow 4 \pi$. If a peak is found in the charged-hadron mass spectrum and if the mass of the missing neutral is consistent with its being a photon (rather than a $\pi^{\circ}$ ), a fairly precise determination of the $x$-mass results by constraining the mass of the parent to be 
$M_{\psi}$, and that of the neutral to be zero. The $X(2830)$ was found by a similar method (61), but with all neutral particles; in particular, this state has been seen only in the decay chain

$$
\psi+\gamma+X(2830) ; \quad X(2830) \rightarrow \gamma \gamma
$$

Therefore, all we know about this meson is that it has even $C$ and cannot have $J^{\mathrm{P}}=I^{ \pm}$.

2) Measurement of the inclusive photon energy distribution in $\psi^{*} \rightarrow \gamma$ + anything and $\psi \rightarrow \gamma+$ anything. Here, the photon is definitely identified and its energy measured, usually with an energy resolution of $(5-10 \%) / \sqrt{E_{\gamma}}$ (in GeV). Peaks in this distribution correspond to $\psi$ or $\psi^{\prime} \rightarrow \gamma+a$ narrow $C=+1$ state. To date, only the states $x(3415), x(3510)$, and $x(3550)$ have been detected by this method (as well as by the other two).

3) Observation of the double-cascade process,

$$
\psi^{\prime} \rightarrow \gamma x ; \quad x+\gamma \psi
$$

Here, both photons are detected, their energies measured, and the $\psi$ is identified by its decay to $\mathrm{e}^{+} \mathrm{e}^{-}$. There is a potential ambiguity in determining the $\chi$ mass because one does not know which photon cane first in a given event of this type: The ambiguity is resolved neatly by plotting the two possible values of the $\psi \gamma$ invariant mass. The wrong solution shows a characteristic Doppler broadening induced by the motion of the $\chi$. In addition to the well-established states at 3414,3508 , and $3552 \mathrm{MeV}$, this method has revealed the existence of a fourth intermediate state, $x(3455)$ (21). Seen in no other way, the only known decay mode of this even-C state is $x(3455) \rightarrow \gamma \psi$. The comparative ease of detecting and identifying $\psi$ and $x$ decay products in $e^{+} e^{-}$antihilation makes it also the best method for determining their spin-parities and branching fractions to individual final states. An 
outstanding example of this is the determination $3^{P C}=1^{--}$for $\psi$ and $\psi^{\prime}$, assignments which are not obvious a priori. This was done by observing the characteristic destructive interference, just below $W=M_{\psi, \psi^{-}}$, between

$$
e^{+} e^{-} \rightarrow Y_{v}+\mu^{+} \mu^{-} \quad\left(Y_{v}=\text { virtual photon }\right)
$$

and

$$
\mathrm{e}^{+} \mathrm{e}^{-} \rightarrow \gamma_{\mathrm{v}} \rightarrow \psi, \psi^{+} \rightarrow \gamma_{\mathrm{v}} \rightarrow \mu^{+} \mu^{-}
$$

The assignments of $\mathrm{J}^{\mathrm{P}}=0^{+}$to $\mathrm{x}_{0}=x(3415), \mathrm{J}^{\mathrm{P}}=1^{+}$to $\mathrm{x}_{1}=x(3510)$, and $J^{P}=2^{+}$to $x_{2}=x(3550)$ are based on the following considerations (62): (i) $x_{0}$ and $x_{2}$ decay to $\pi^{+} \pi^{-}$and $\mathrm{K}^{+} \mathrm{K}^{-}$and, therefore, both states have natural spin-parity; these modes have not been observed for $x_{1}$, consistent with the $1^{+}$ assignment. (ii) the angular distribution of the photon in $\psi^{-} \rightarrow \gamma x_{0}$ is wellfit by $1+\cos ^{2} \theta$, which is expected for $J=0$. The angular distributions $1-1 / 3 \cos ^{2} \theta$ for $J=1$ and $I+\frac{1}{13} \cos ^{2} \theta$ for $J=2$ are consistent with the rather meager measurements for $\chi_{1}$ and $x_{2}$, respectively.

Finally, the normal hadronic widths, $\sim 10^{\prime} \mathrm{s}$ of $\mathrm{MeV}$, of the directly-produced resonances above $\psi^{-(3684)}$ are further dramatic confirmation of the OZI rule. Here, the charmed quarks need no longer annihilate since it is energetically possible for them to emerge (together with light quarks) as the charmed mesons $D, D^{*}, F, F^{*}$, and so on. All this will be discussed in Section IV. Suffice it to say that these broad charmonium resonances were solely responsible for the unambiguous isolation of charmed mesons.

\section{B. FOUNDATIONS OF THE CHARMONIUM MODEL}

Perhaps the most important feature of the charmonium spectrum in Fig. 2 is the fact that the level spacings are very small compared to the overall mass scale of the system. This suggests, at least for the states below charm threshold, that the system is nonrelativistic, with excitation energies small compared to 
the masses of the constituents. This is something completely new in strong interaction physics, and a great deal of theoretical work has gone into analyzing this system using a nonrelativistic schrödinger equation formalism $(63,64)$.

In retrospect, this approach is somewhat too naive, especially with regard to the assumption of spin independence of the dominant $\bar{c} \bar{c}$ interaction. The hyperfine splitting is not much smaller than the radial and orbital excitation energies. Nevertheless, the model has, at the very least, been a powerful predictive guide to the qualitative features of charmed quark physics.

The other aspect of the charmonium model is the attempt to understand the narrowness of the states below charm threshold as a consequence of asymptotic freedom. We sha11 now discuss the theoretical basis for this possibility in some detail, but it is important to keep in mind that it is largely a separate issue from the energy level structure. There, it is clear from Fig. 2 that without a solution to QCD in the strong coupling regime, some phenomenological input is necessary. In the case of the decay widths, there is the possibility that perturbation theory may be directiy relevant.

The idea is basically that with $\overline{c c}$ annihilation into light hadrons proceeding through gluons, the decay will be inhibited since the effective gluon coupling constant should be small at high energies $(47,65)$. The dominant contribution will come from the minimum number of intermediate gluons which depends upon the quantum numbers of the charmonium state. Some rather striking experimental predictions can be made on the basis of this "gluon counting"; these will be discussed in Sec. III.C.

Consider the decay of the $\psi\left({ }^{3} \mathrm{~S}_{1}\right)$ state. Its dominant electromagnetic decay is shown in Fig. 3. The $\overline{C C}$ pair must come together to annihilate into the virtual photon, and if the bound state is nonrelativistic, then, to first 
approxination, the decay width will be given by

$$
\Gamma\left(\psi \rightarrow \ell^{+} \ell^{-}\right)=\frac{4 \alpha^{2}(2 / 3)^{2}}{M^{2}}|\Psi(0)|^{2}
$$

The charge of the charmed quark is $2 / 3|e|$, and $M$ is the charmonium mass. $\Psi(0)$ is the nonrelativistic radial wave function at the origin, and one cannot expect to be able to compute it in perturbation theory. The reason for this is that the mean radius $\langle x\rangle$ of charmonium is on the order of one fermi, a distance scale at which the effective coupling strength for the binding has become large. Thus, $\Psi(0)$ will be determined in part by the nonperturbative, long range part .of the potential. The hadronic decay of the $\psi$ must proceed through a minimum of three giuons. If this is indeed the dominant contribution, that is to say, if perturbation theory is truly relevant to this problem, the decay will proceed as shown in Fig. 4. The $\bar{c}$ annihilation will be essentially local (on the order of $1 / \mathrm{m}_{\mathrm{c}}\langle\langle\zeta\rangle)$. The computation of the decay matrix element is then done in analogy to the parton model computation of $\sigma_{\text {tot }}\left(e^{+} e^{-} \rightarrow\right.$ hadrons) as if the final state consisted of three on-mass-shell gluons. This amounts to the statement that the transition from the three gluon state into physical hadrons takes place with unit probability. A more satisfying theoretical justification of the three gluon mechanism can be given and we will turn to it shortly. If the mechanism just described is correct, then the total hadronic width of the $\psi$ is given by (65).

$$
\Gamma(\psi \rightarrow \text { hadrons })=\frac{40}{81 \pi}\left(\pi^{2}-9\right) \frac{\alpha_{s}^{3}\left(M^{2}\right)}{M^{2}}|\Psi(0)|^{2}
$$

The strong coupling constant is defined at the $\psi$ mass and, as before, $\psi(0)$ is the nonrelativistic wave function at the origin.

Before proceeding to the comparison of these expressions with experiment, we sketch the analysis that underlies Eq. 3.7. A necessary condition for 
the applicability of lowest order perturbation theory is that no large dynamical factors enter in higher orders to make the expansion break down. One must analyze the quantity $\sum_{n}\left|M_{c} \bar{c} \rightarrow n\right|^{2}$ where $n$ is some quark-gluon final state. $M$ is the decay matxix element and is defined to be two-particle (c $\bar{c}$ ) irreducible in the decay channel. Two-particle reducible contributions are absorbed into the definition of the wave function. If it can be shown that $\sum_{n}\left|M_{c} \bar{c} \rightarrow n\right|^{2}$ is free of infrared singularities for the $\bar{c} \bar{c}$ pair at rest, then there can be no large dynamical factors. This is because the result will involve no small energy or momentum factors, only the (large) charm quark mass and the (large) renormalization scale. The infrared finiteness through order $\alpha_{s}^{4}$ (the lowest order is $\alpha_{s}^{3}$ ) for $\psi$ decay has been checked and it is conjectured to be true to all orders. It is technically simpler to use the Coulomb rather than covariant gauges in this analysis.

We make one last point before proceeding. The infrared analysis is necessary but not sufficient. It could we11 be that threshold singularities in high orders, or even completely nonperturbative effects prevent the use of perturbation theory in this simple way. The use of asymptotic freedom to explain the OZI rule is speculative. It is on much less solid footing than the conventional deep Euclidean application or even the direct computation of $\sigma_{\text {tot }}\left(e^{+} e^{-} \rightarrow\right.$ hadrons $)$, since there the production is truly local, coming from the off-shell photon. 


\section{THE BASIC CHARMONIUM MODEL}

To go beyond qualitative predictions, a model of the $\mathrm{c}-\overline{\mathrm{c}}$ interaction is necessary, and it is natural to realize this in terms of an instantaneous, central potential, $V_{0}(r)$. Such a model presupposes that, to a first approximation, one may neglect the influences of spin-dependent forces and of nearby open decay channels on spectroscopy and decay rates. The attempts to incorporate these effects in the basic model will be described in Sections IIr. $D$ and $E$.

A simple possibility for $V_{o}$, motivated by asymptotic freedom at short $c-\bar{c}$ separations and quark confinement at large ones, is $(66,63,64)$.

$$
v_{0}(x)=-\frac{k}{r}+\frac{r}{a^{2}}
$$

Asymptotic freedom tells us to expect a rather small short-distance coupling, say $k \approx 0.2$ The choice of 1inear confining term is suggested by the lattice gauge theory $(51,52)$ and the dual string model $(67)$. Then $1 / a^{2}$ is related to the Regge slope and is $1 / \mathrm{a}^{2} \simeq 1 \mathrm{GeV} / \mathrm{fm} \simeq 0.2(\mathrm{GeV})^{2}$. The third parameter of this model is the charmed quark mass, which is expected to be $\mathrm{m}_{\mathrm{c}} \simeq \mathrm{M}_{\psi} / 2 \simeq 1.5 \mathrm{GeV}$. We emphasize that these parameters are purely phenomenological. For example, $m_{c}^{-1}$ is really the strength of the kinetic energy term in the c $\bar{c}$ Hamiltonian, and not necessarily equal to half the difference between the mass of a state and its energy eigenvalue. ${ }^{6}$ Thus, within the general guidelines set by the above expectations, the three parameters will be determined by fitting to se*lected pieces of data. When this mode1 is applied to bound systems of heavier quarks, the $T$ for example, it is presumed that only the quark mass will change substantially; $K$ may decrease slightly, while the linear potential strength $a^{-2}$ is thought to be independent of the quark mass. (37).

A final word about this choice of $\mathrm{v}_{\mathrm{o}}$ : From a purely phenomenological point of view, other forms may be equally reasonable and give as acceptable an account 
of the data. The advantages of $\mathrm{v}_{0}$ are that it is we11-motivated and that it contains the minimum number of parameters needed to reach agreement with the observed spectra and decay rates in charmonium. There is evidence that $\mathrm{V}_{0}$ does not adequately describe the $T$ spectrum $(23,57)$, and some possible modifications will be discussed in Sec. III.F.

To obtain a "best" overa11 description of charmonium data, Eichten et al $(63,68)$ choose parameters $k, a$, and $m_{c}$ by (1) fitting to the $\psi^{\prime}-\psi$ mass difference, (2) taking the electronic width of $\psi$ to be $5.3 \mathrm{kev}$, one standard deviation above the measured value of $4.8 \pm 0.5 \mathrm{keV}$, and (3) constraining $1.5 \mathrm{GeV}$ $\lesssim \mathrm{m}_{\mathrm{c}} \leq 2.0 \mathrm{GeV}$ and $0.1 \leq k \leq 0.4$. These constraints are consistent with the notion of heavy constituents moving nonrelativistically and with weak shortrange interactions. Acceptable results are obtained with a range of parameters, and their preferred choice is

$$
m_{c}=1.65 \mathrm{GeV}, \quad \mathrm{a}=2.07 \mathrm{GeV}^{-1}, \quad k=0.132
$$

A check on the self-consistency of the nonrelativistic approximation is provided by the mean-squared quark velocity in the $1 \mathrm{~S}$ and $2 \mathrm{~S}$ states; these are $\left\langle v^{2}\right\rangle=0.17$ and 0.28 , respectively. It is worth emphasizing that the Coulomb part of the potential, although the most certain feature according to QCD, may not be very meaningful for charmonium. For this value of $k$, it only becomes important below distance scales $v_{\mathrm{m}}^{-1}$, where a nonrelativistic potential picture ceases to be sensible.

CHARMONIUM SPECTRUM The most important consequence of the existence of a confining potential (of almost any shape) between $c$ and $\bar{c}$ is that there will be ${ }^{3}$ p-states Iying between the ${ }^{3}$ s-states, $\psi$ and $\psi^{\prime}(63,64,69,70)$. The reason for this is simple. Recalling that, in a purely Coulomb potential, the $2 \mathrm{~S}$ and $1 \mathrm{P}$ states are degenerate, it is clear that the presence of a confining term will impart 
a greater kinetic energy to the $2 \mathrm{~S}$ state, with its one radial node, than to the $1 \mathrm{P}$, which has only an orbital node, thus lifting the degeneracy. The same remark applies to the relative ordering of $3 \mathrm{~S}, 2 \mathrm{P}$, and 10 states, and so only the amount of this splitting depends on details of the potential and quark mass. In charmonium, we are in an extremely fortunate position to observe this consequence of quark confinement because, lying below the ozI-allowed decay threshold, $2 \mathrm{M}_{\mathrm{D}}$, the $1^{3} \mathrm{P}$ states, 1ike $\psi_{\text {and }} \psi^{*}$, will be unusually narrow. Furthermore, they have even charge conjugation $(C=+1)$, and so may be detected by radiative transitions from $\psi^{\prime}$ and, if narrow enough, down to $\psi$. Again, branching ratios will depend on details of the model, but the existence of these states and their mode of observation is inescapable.

Using the potential $V_{0}$ with parameters Eq. (3.9), the Schrodinger equation nay be numerically integrated to obtain the spectrum of low-lying spin-triplet states shown in Table 2 (68), together with the most likely candidates for these states. A number of explanatory remarks are in order: (1) For brevity only those states even remotely accessible to existing experimental techniques have been included. (2) With the neglect of spin-dependent forces, spin-singlet states such as $n_{c}=I^{1} S_{0}$ and $I^{1} P_{1}$ would be degenerate with the predicted center-of-gravity (c.o.g.) of the corresponding triplet states. And, by adjusting the constant zero of energy to give the correct $\psi$ mass, that constant subsumes some of the hyperfine interaction $\left(\propto \vec{S}_{1} \cdot \vec{S}_{2}\right.$ ) for the low-lying ${ }^{3}$ S-states. (3) As noted previously, the evidence is fairly strong in favor of the assignment of $x_{0}=x(3415)$, $x_{1}=x(3510)$, and $x_{2}=x(3550)$ to $1^{3} \mathrm{P}_{0,1,2}$ respectively; the c.0.g. of these levels is $3522 \pm 5 \mathrm{MeV}$, somewhat higher than predicted. (4) Most model calculations of the splitting among the $1^{3} \mathrm{D}_{\mathrm{J}}$ levels expect it to be smaller than that observed for the $1^{3} \mathrm{P}_{\mathrm{J}}$ (see the next section), so that al1 three of these $\mathrm{L}=2$ states may be fairly close to the mass of $\psi(3772)=1^{3} D_{1}$. (5) The region 
Table 2.

Predicted (68) and observed spin-triplet charmonium levels. Assignment of $\psi(4028)$ to $3^{3} \mathrm{~S}_{1}$, existence of $\psi(4160)$ and its assignment to $2^{3} \mathrm{D}_{1}$ are open to question.

\begin{tabular}{|c|c|c|}
\hline State $n^{3} L_{J}\left(J^{P C}\right)$ & Predicted Mass (MeV) & Candidate (Measured Mass) \\
\hline $1^{3} S_{1}\left(1^{--}\right)$ & 3095 (Input) & $\psi(3095)$ \\
\hline $1^{3} \mathrm{P}_{\mathrm{J}}\left(0^{++}, 1^{++}, 2^{++}\right)$ & 3457 & $x_{0,1,2}(3522 \pm 5)$ \\
\hline$s_{1}\left(1^{-\infty}\right)$ & 3684 (Input) & $\psi^{-}(3684)$ \\
\hline $1^{3} \mathrm{D}_{\mathrm{J}}\left(1^{--}, 2^{--}, 3^{--}\right)$ & 3755 & $\psi(3772)=1^{3} D_{1}$ \\
\hline $2^{3} \mathrm{P}_{\mathrm{J}}\left(0^{++}, 1^{++}, 2^{++}\right)$ & 3957 & $\begin{array}{l}\text { Above charm threshold; } \\
\text { difficult to produce. }\end{array}$ \\
\hline $3^{3} s_{1}\left(1^{--}\right)$ & 4.157 & $\psi(4028)$ \\
\hline $2^{3} \mathrm{D}_{\mathrm{J}}\left(1^{--}, 2^{--}, 3^{--}\right)$ & 4204 & $\psi(4160)=2^{3} D_{1}$ \\
\hline $4^{3} s_{1}\left(1^{--}\right)$ & 4567 & $\psi(4414)$ \\
\hline
\end{tabular}


between c.m. energy $W=4.0$ and $4.3 \mathrm{GeV}$ in $\mathrm{e}^{+} \mathrm{e}^{-}$annihilation is quite complicated (see Sec. III.E) and difficult to interpret. It is obvious that the peak $\psi(4028)$ is a resonance and, within the charmonium model, is assigned to the $3^{3} \mathrm{~S}_{1}$ leve1. If it should become equally clear that the enhancement at $\mathrm{W} \simeq 4.16 \mathrm{GeV}$ is a resonance, the candidate assignment is $2^{3} \mathrm{D}_{1}$. That both these states and the $\psi(4414)$, assigned to $4^{3} S_{1}$, appear $\approx 150 \mathrm{MeV}$ lower than predicted shows that the approximations underlying the model are breaking down. Another sign of this is the sizeable electronic width, $2.2-.4 \mathrm{keV}$, of $\psi(3772)$. In the nonrelativistic potential model, $T_{e} \propto|\psi(0)|^{2}$ vanishes for all but ${ }^{3} S_{1}$ states. The observed electronic width is fairly well understood as a coupled-channel effect, and will be discussed in Sec. III.E.

CHARMONIUM TRANSITION RATES A great deal can be said about the decay rates and branching ratios of the charmonium levels (below $\bar{D} \bar{D}$ threshold), most of which requires some knowledge of the bound state wave functions. The rates that can be computed in the nonrelativistic model fall into two classes: those that depend on the wave function at very short $\bar{c}$ separations (electronic, two-photon, and the hadronic widths obtained from gluon counting), and those that involve overlaps of radial wave functions (EI and $M I$ radiative widths). The reader should be aware of what is being neglected in these calculations. Those involving the wave function $\Psi_{\mathrm{nL}}(r)$ near $\mathrm{r}=0$ certainly ignore possibly important relativistic and quantum effects such as spin dependence and pair creation, as well as the mixing among states (e.g. $2^{3} \mathrm{~S}_{1}$ and $1^{3} \mathrm{D}_{1}$ ) induced by coupling to decay channels. The second type of calculation does not include almost certain reductions in the overlap integral due to differing gluon distributions in the initial and final states, nor does it take account of the spin-dependent and mixing effects noted above. Finally, the charmonium model has very Iittle to say on the important question of $\psi^{-} \rightarrow \psi \pi \pi$ (and $T^{-} \rightarrow T \pi \pi$, $T n$ ) because the rather 
low momentum imparted to the $\pi \pi$ or $\eta$ makes gluon counting especially dubious. See, however, Gottfried (71) for scaling rules for these decays.

The leptonic decay width is readily computed from the graph in Fig 3 . If $Q$ is the charge of the quark $q, M_{n 0}$ the mass of the $J^{P C}=1^{--}$states $n^{3} S_{1}$, $\Psi_{n 0}(0)$ its radial wave function at the origin, and $m_{\ell}$ the mass of the charged lepton, the result is (72)

$$
r\left(n^{3} s_{1} \rightarrow \ell^{+} \ell^{-}\right)=4 Q^{2} \alpha^{2} \frac{\left|{ }_{n} 0^{(0)}\right|^{2}}{M_{n 0}^{2}}\left(1+\frac{2 m_{\ell}^{2}}{M_{n 0}^{2}}\right)\left(1-\frac{4 m_{\ell}^{2}}{M_{n 0}^{2}}\right)^{\frac{1}{2}}
$$

The terms involving $m_{\ell}$ are relevant for decays such as $\psi^{-} \rightarrow \tau^{+} \tau^{-}$and $T^{-} \tau^{+} \tau^{-}$.

The rates for the ozI-forbidden direct hadronic decays of heavy-quark systems via antihilation to the minimum possible number of gluons can be computed from graphs as in Fig. 4. The results are (to lowest order in quark velocity) $(65,73)$

$$
\begin{aligned}
& \Gamma\left(n^{3} S_{1} \rightarrow \text { hadrons }\right)=\frac{40}{81 \pi}\left(\pi^{2}-9\right) \alpha_{s}^{3} \frac{\left|\psi_{n 0}(0)\right|^{2}}{M_{n 0}^{2}}, \\
& \Gamma\left(\mathrm{n}^{1} \mathrm{~S}_{0} \rightarrow \text { hadrons }\right)=\frac{8}{3} \alpha_{\mathrm{s}}^{2} \frac{\left|\Psi_{\mathrm{n} 0}(0)\right|^{2}}{\mathrm{M}_{\mathrm{n} 0}^{2}}, \\
& \Gamma\left(n^{3} \vec{P}_{0} \rightarrow \text { hadrons }\right)=96 \alpha_{s}^{2} \frac{\left|y_{n 1}^{-}(0)\right|^{2}}{M_{n I}^{4}}, \\
& \Gamma\left(n^{3} P_{1}+\text { hadrons }\right)=\frac{128}{3 \pi} \alpha_{s}^{3} \frac{\left|\Psi_{n 1}^{\prime}(0)\right|}{M_{n 1}^{4}} \ln \left(\frac{4 m_{q}^{2}}{4 m_{q}^{2}-M_{n 1}^{2}}\right) \text {, } \\
& \Gamma\left(\mathrm{n}^{3} \mathrm{P}_{2} \rightarrow \text { hadrons }\right)=\frac{128}{5} \alpha_{\mathrm{s}}^{2} \frac{\left|\psi_{\mathrm{n} 1}^{\prime}(0)\right|^{2}}{\mathrm{M}_{\mathrm{n} 1}^{4}}, \\
& \Gamma\left(n^{1} p_{1} \rightarrow \text { hadrons }\right)=\frac{320}{9 \pi} \alpha_{s}^{3} \frac{\left|\psi_{n l}^{\prime}(0)\right|^{2}}{M_{n 1}^{4}} \ln \left(\frac{4 m_{q}^{2}}{4 m_{q}^{2}-m_{n 1}^{2}}\right) .
\end{aligned}
$$


$\alpha_{s}$ is evaluated at the bound state mass $M_{n L}$, and $\psi_{n L}^{\prime}(0)$ is the slope of the radial wave function at $r=0$. The rate for $\psi_{\mathrm{nL}}$ to decay directiy to a photon plus hadrons may also be obtained from gluon counting; the formula is $(69,74)$

$$
\Gamma\left(n^{3} S_{1} \rightarrow \gamma+\text { hadrons }\right)=\frac{32}{9 \pi}\left(\pi^{2}-9\right) \alpha_{s}^{2} \alpha Q^{2} \frac{\left|\psi_{n 0}(0)\right|^{2}}{M_{n 0}^{2}}
$$

Two more direct decay widths, not involving gluon counting but of great importance nonetheless, are

$$
\begin{aligned}
& \Gamma\left(n^{3} S_{1} \rightarrow \gamma \rightarrow \text { hadrons }\right)=R_{B K G D .} \Gamma\left(n^{3} S_{1} \rightarrow e^{+} e^{-}\right) \\
& \Gamma\left(n^{1} S_{0} \rightarrow \gamma \gamma\right)=12 Q^{4} \alpha^{2} \frac{\left|{ }_{n O}(0)\right|^{2}}{M_{n 0}^{2}} \\
&=\frac{9}{2} Q^{4}\left(\frac{\alpha}{\alpha_{s}}\right)^{2} \Gamma\left(n^{1} S_{0} \rightarrow \text { hadrons }\right)
\end{aligned}
$$

where $R_{B K G D}$. is the value of $R$ just off the resonance peak.

Note that Eq. (3.19a) involves only the short distance (positronium-1ike) assumption, while Eq. (3.19b) involves the much stronger gluon-counting assumption. The two-photon width of ${ }^{3} \mathrm{P}_{0}$ and ${ }^{3} \mathrm{P}_{2}$ states is related to their hadronic (2 gluon) widths by the same factor, $9 Q^{4} \alpha^{2} / 2 \alpha{ }^{2}$, as for ${ }^{1} S_{0}$ states. To the extent that wave functions are independent of total quark spin and angular momentum $\vec{J}=\overrightarrow{\mathrm{L}}+\overrightarrow{\mathrm{S}}$ (for fixed $\mathrm{n}, \mathrm{L}$ ), we have

$$
\Gamma\left(n^{3} S_{1} \rightarrow \text { hadrons }\right) / \Gamma\left(n^{1} S_{0} \rightarrow \text { hadrons }\right)=\frac{5\left(\pi^{2}-9\right) \alpha}{27 \pi}
$$

and

$$
\begin{aligned}
& \Gamma\left(\mathrm{n}^{3} \mathrm{p}_{0} \rightarrow \text { hadrons }\right): \Gamma\left(\mathrm{n}^{3} \mathrm{p}_{1} \rightarrow \text { hadrons }\right): \Gamma\left(\mathrm{n}^{3} \mathrm{p}_{2} \rightarrow \text { hadrons }\right) \\
& \quad=1: \frac{4 \alpha s}{9 \pi} \ln \left(\frac{4 \mathrm{~m}_{\mathrm{q}}^{2}}{4 \mathrm{~m}_{\mathrm{q}}^{2}-\mathrm{M}_{\mathrm{n} I}^{2}}\right): \frac{4}{15}
\end{aligned}
$$


The formulae Eq. (3.14) and (3.16) for ${ }^{3} \mathrm{P}_{1},{ }^{1} \mathrm{P}_{1} \rightarrow$ hadrons deserve some comment. In the spirit of gluon counting, a spin-one state cannot decay to two massless, on-she11 gluons, and so we expect these rates to be $0\left(\alpha_{S}^{3}\right)$, not $0\left(\alpha_{S}^{2}\right)(73,75)$. In particular, $X_{I}$ should be the narrowest of the ${ }^{3} P_{J}$ levels. In an actual calculation of these rates (73), the dominant contribution involves the exhibited logarithn, due to a threshold singularity at $2 \mathrm{~m}_{c}=\mathrm{r}_{\mathrm{X}_{1}}$. Such a large logarithm is always worrisome in QCD calculations, since it may signal the breakdown of perturbation theory. So it is difficult to take these results too seriously beyond the reasonable (and conservative) guess that $\Gamma\left({ }^{3} \mathrm{P}_{1} \rightarrow\right.$ hadrons) is $\approx \alpha_{s} \Gamma\left({ }^{3} \mathrm{P}_{0,2} \rightarrow\right.$ hadrons $)$.

The predictions of these formulae for the charmonium system are listed in Table 3. Experimental comparisons are best delayed until the discussions of radiative transition rates. The value of $\alpha_{s}$ used here is determined by fitting to the total width of $\psi$,

$$
\begin{aligned}
\Gamma(\psi \rightarrow \mathrm{a} 11) & \triangleq \Gamma\left(\psi \rightarrow \mathrm{e}^{+} \mathrm{e}^{-}\right)\left[2+\mathrm{R}_{\mathrm{BKGD}}\right]+\Gamma(\psi \rightarrow \text { hadrons })+\Gamma(\psi \rightarrow \gamma+\text { hadrons }) \\
& =(69 \pm 15) \mathrm{keV},
\end{aligned}
$$

where the small width for $\psi \rightarrow \gamma X(2830)$ has been ignored. Using $R_{B K G D} \cong 2.2$ $(58,59)$ and $\Gamma\left(\psi \rightarrow e^{+} e^{-}\right)=5.3 \mathrm{keV} \mathrm{gives}^{7}$

$$
\alpha_{s}\left(M_{\psi}\right) \approx 0.19
$$

The wave functions for states other than $\psi$ are determined using the parameters in Eq. (3.9).

The important qualitative features of these calculations are: (1) The ground-state pseudoscalar $\eta_{c}$ is expected to have a total width in the $\mathrm{MeV}$ range, about 100 times greater than the width of $\psi$, and its branching ratio 
Table 3

Direct decays in charmonium, calculated from Eqs. 3.10-3.21. $\Gamma\left(X_{J} \rightarrow a 11\right)=$ $\Gamma\left(X_{J} \rightarrow\right.$ hadrons $)+\Gamma_{Y}\left(X_{J} \rightarrow \psi\right), \Gamma\left(\eta_{C}^{\prime} \rightarrow\right.$ all $)=\Gamma\left(n_{C}^{\prime} \rightarrow\right.$ hadrons $)+\Gamma\left(\eta_{c}^{-} \rightarrow \eta_{c} \pi \pi\right)$, and $\Gamma\left(\eta_{c}^{\prime} \rightarrow \pi_{c} \pi \pi\right)=\Gamma\left(\psi^{\prime} \rightarrow \psi \pi \pi\right)$ are assumed.

$\begin{array}{lcc}\text { Mode } & \text { Rate }(\mathrm{keV}) & \text { Branching Ratio }(\%) \\ \eta_{c} \rightarrow \text { hadrons } & 5.1 \times 10^{3} & 100 \\ \eta_{c} \rightarrow \gamma \gamma & 7.1 & 0.14 \\ \psi \rightarrow \gamma+\text { hadrons } & 6.1 & 8.0 \\ \chi_{0} \rightarrow \text { hadrons } & 1.8 \times 10^{3} & 90 \\ \chi_{0} \rightarrow \gamma \gamma & 2.5 & 0.13 \\ x_{1} \rightarrow \text { hadrons } & 105 & 21 \\ \chi_{2} \rightarrow \text { hadrons } & 480 & 48 \\ x_{2} \rightarrow \gamma \gamma & 0.66 & 6.6 \times 10^{-2} \\ \eta_{c}^{\prime} \rightarrow \text { hadrons } & 3.3 \times 10^{3} & 97 \\ \eta_{c}^{\prime} \rightarrow \gamma \gamma & 4.5 & 1.7 \\ \psi^{-} \rightarrow \text { hadrons } & 31 & 14 \\ \psi^{\prime} \rightarrow \gamma+\text { hadrons } & 3.9 & 0.13\end{array}$


to two photons should be $\sim 10^{-3}$. Similar remarks apply to its first radial excitation $n_{c}^{-}$, which may also decay to $n_{c}+\pi \pi$ (presumably, $\Gamma\left(n_{c}^{\prime} \rightarrow n_{c} \pi \pi\right)$ $\left.\simeq \Gamma\left(\psi^{*} \rightarrow \psi \pi \pi\right)\right)$. (2) As discussed above, gluon counting implies that ${ }^{3} P_{I}$ and ${ }^{I_{P_{1}}}$ have considerably less hadronic width than ${ }^{3} \mathrm{P}_{0}$ and ${ }^{3} \mathrm{P}_{2}$. An immediate consequence is that the branching ratio $B\left(\psi^{\prime} \rightarrow X_{J} \gamma\right) B\left(x_{J} \rightarrow \psi \gamma\right)$ should be largest for the $1^{H}$ state $x_{1}$. (3) The potential model predicts $\Gamma\left(\psi^{\prime} \rightarrow \mathrm{e}^{+} \mathrm{e}^{-}\right)=3.4 \mathrm{keV}$ and $\Gamma\left(\psi^{\prime} \rightarrow 3\right.$ gluons $\rightarrow$ hadrons $)=31 \mathrm{keV}$,

both in fair agreement with the measured values (59), $2.0 \mathrm{keV}$ and $\sim 20 \mathrm{keV}$. respectively. These results lend some support to both the presence of a linear confining term in $V_{0}$ (since for $k=0,\left|\Psi_{10}(0)\right|=\left|\Psi_{20}(0)\right|$ ) and to the gluoncounting calculation of the direct hadronic width.

We turn now to the radiative decays. The El transition rate between S- and $p$-wave states having the same total quark spin is.

$$
\Gamma_{\gamma}(S \leftrightarrow P)=\frac{4}{9}\left(\frac{2 J_{f}+1}{2 J i j}\right) Q^{2} \alpha\left|E_{i f}\right|^{2} \omega^{3},
$$

where $w$ is the photon energy, $J_{i}\left(J_{f}\right)$ the total angular momentum of the initial (fina1) state, $Q=\frac{2}{3}$ for charmed quarks, and $\mathrm{E}_{\text {if }}$ is the transition dipole matrix element,

$$
E_{i f}=\int_{0}^{\infty} r^{2} d r_{i}^{\Psi}(r) \Psi_{f}(r) r
$$

Here, $\Psi_{i, f}$ are initial (final) state radial wave functions. El rates between ${ }^{3} \mathrm{D}_{1}$ and ${ }^{3} \mathrm{P}_{\mathrm{J}}$ states axe given by

$$
\Gamma_{Y}\left({ }^{3} D_{1} \leftrightarrow{ }^{3} P_{J}\right)=\frac{4}{9}\left(\frac{2 J_{f}+1}{2 J_{i}+1}\right) D_{i f} Q^{2} \propto\left|E_{i f}\right|^{2} \omega^{3}
$$


where $D_{\text {if }}=1,1 / 4,1 / 100$ for $J=0,1,2$ respectively. For the charmonium system, the $I^{3} D_{1}$ state lies above charm threshold and so it has a very small branching ratio to $x_{\mathrm{J}}+\gamma$. Therefore, Eq. (3.26) is useful only indirectly, through the mixing between $2^{3} \mathrm{~S}$, and $1^{3} \mathrm{D}_{1}$ (see Sec. III.E). In the $T$ and heavier quark bound systens, however, there is an excellent chance for direct observation of the ${ }^{3} \mathrm{D}_{1} \leftrightarrow{ }^{3} \mathrm{P}_{\mathrm{J}}$ El transitions.

The $\mathrm{Ml}$ transition rate between ${ }^{3} \mathrm{~S}_{1}$ and ${ }^{1} \mathrm{~S}_{0}$ states is taken to be

$$
\Gamma_{\gamma}\left({ }^{3} S_{1} \leftrightarrow{ }^{1} S_{0}\right)=\frac{16}{3}\left(2 J_{f}+1\right)\left(\frac{Q}{2 m_{G}}\right)^{2} \alpha\left|M_{i f}\right|^{2} \omega^{3}
$$

where a Dirac moment is assumed for the quark, and

$$
M_{i f}=\int r^{2} d r j_{0}(w r / 2) \Psi_{i}(r) \Psi_{f}(r)
$$

For the "allowed" Ml transitions between hyperfine partners, $M_{\text {if }}$ is very nearly unity because $\Psi_{i}=\Psi_{f}$ and $w\left\langle r_{i f} / 2 \ll 1\right.$, so that $j_{0} \simeq 1$. For the same reason $M 1$ transitions between $S$ states corresponding to different radial quantum numbers $\mathrm{n}_{i} \neq \mathrm{n}_{\mathrm{f}}$ are strongly suppressed. It is still true that $\omega\langle r\rangle_{\text {if }} / 2 \ll 1$, and

$$
M_{i f} \cong-\frac{\omega}{24} \int r^{2} \mathrm{dr} \Psi_{i}(r) \Psi_{E}(r) r^{2} \ll 1
$$

The immediate consequence is that the "hindered" M1 transitions $\psi^{-}\left(2^{3} \mathrm{~S}_{1}\right) \rightarrow$ $n_{c}\left(1^{1} S_{0}\right)+\gamma$ and $n_{c}^{\prime}\left(2^{1} s_{0}\right) \rightarrow \psi\left(1^{3} S_{1}\right)+\gamma$ are expected to be very rare compared to allowed MI and $\mathrm{El}$ transitions.

The predictions of the potential model for $\mathrm{El}$ rates and branching fractions are compared with experimental observations in Table 4. Following the custom of traditional spectroscopy, experimental values of the $x$ masses are used so that what is being tested here is the theoretical strength $\Gamma \gamma^{/ \omega^{3}}$. 
Table 4

El decays in charmonium for theory (68) and experiment (59). Predicted total widths for $x_{0,1,2}$ of $2.0,0.5$ and $1.0 \mathrm{MeV}$, respectively have been assumed.

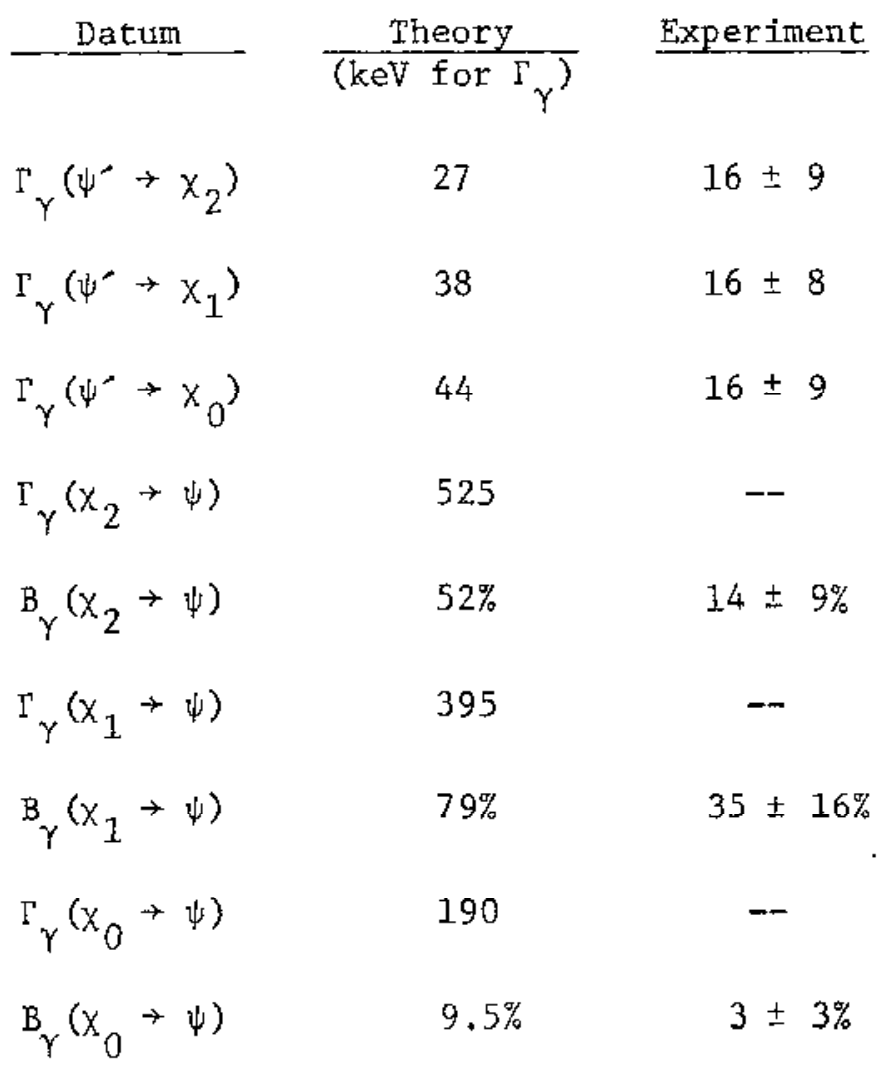


Given the naivete of the simple potential model, the agreement is rather good, with theory lying within 1-2 standard deviations of experiment. Especially noteworthy are: (1) The normalized experimental rates are (with large errors) (59)

$$
\frac{\Gamma\left(\psi^{-} \rightarrow \gamma x_{2}\right)}{5 \omega_{2}^{3}}: \frac{\Gamma\left(\psi^{-} \rightarrow \gamma x_{1}\right)}{3 \omega_{1}^{3}}: \frac{\Gamma\left(\psi^{\circ} \rightarrow \gamma x_{0}\right)}{\omega_{0}^{3}}=1: 0.7: 0.6,
$$

with unity expected for pure El transitions from ${ }^{3} \mathrm{~S}_{1}$ to ${ }^{3} \mathrm{P}_{\mathrm{J}}$ states. (2) The measured branching ratios for $x_{J} \rightarrow \gamma \psi$ are quite consistent with predictions based on both the potential model and gluon counting for the $x$ hadronic widths, with $\chi_{1}$ considerably more narrow than $x_{0}$ and $x_{2}$. These facts strengthen the $J^{\mathrm{P}}$ assignments discussed in Sec. III.A.

The Ml transition rates are compared with experiment in Table 5, where we have made the tentative assignments of $X(2830)=n_{c}\left(1^{1} S_{0}\right)$ and $x(3455)=\pi_{c}^{\prime}\left(2^{1} S_{0}\right)$. If these identifications turn out to be correct, they will represent a serious failure of the charmonium model:

(1) From Table 1 ,

$$
\begin{aligned}
& \mathrm{B}(\psi \rightarrow \mathrm{X}(2830) \gamma) \mathrm{B}(\mathrm{X} \rightarrow \gamma \gamma)=1.3 \pm 0.4 \times 10^{-4} \\
& \mathrm{~B}(\psi \rightarrow \mathrm{X}(2830) \gamma)<.017(90 \% \text { confidence level })
\end{aligned}
$$

From these, one infers

$$
B(X(2830) \rightarrow \gamma \gamma) \approx 7 \times 10^{-3}
$$

which is at least a factor of five larger than the predicted value (Table 3 ). This is to be contrasted with the apparent success of calculated direct decay rates for the ${ }^{3} P$ states and for $\psi^{\prime}$.

(2) The model fails by at least an order of magnitude in predicting 
Table 5

Ml decays in charmonium. Observed total widths of $\psi^{\prime}$ and $\psi^{\prime}$ (Table 1) and predicted total widths of $\eta_{c}$ and $\eta_{c}^{-}$(Table 2) are used in determining branching ratios.

\begin{tabular}{|c|c|c|}
\hline Datum & Theory & Experiment \\
\hline$\Gamma_{Y}\left(\psi \rightarrow n_{c}\right)$ & $26 \mathrm{keV}$ & $<1.2 \mathrm{keV}$ \\
\hline$B_{\gamma}\left(\psi \rightarrow n_{c}\right)$ & $37 \%$ & $<1.7 \%$ \\
\hline $\mathrm{B}_{\gamma}\left(\psi \rightarrow \eta_{c}\right) \mathrm{B}\left(\eta_{c} \rightarrow \gamma \gamma\right)$ & $0.052 \%$ & $0.013 \pm 0.004 \%$ \\
\hline$\Gamma_{\gamma}\left(\psi^{\prime} \rightarrow \eta_{c}\right)$ & $1.9 \mathrm{keV}$ & $<2.3 \mathrm{keV}$ \\
\hline$\left.{ }^{B} \gamma^{\left(\psi^{\prime}\right.} \rightarrow n_{c}\right)$ & $0.83 \%$ & $<3.0 \%$ \\
\hline${ }_{\gamma}^{B_{\gamma}}\left(\psi^{\prime} \rightarrow n_{c}\right) B\left(n_{c}+\gamma \gamma\right)$ & $1.2 \times 10^{-3} \%$ & $<3.4 \times 10^{-2} \%$ \\
\hline$\Gamma_{\gamma}\left(\psi^{\prime}+\eta_{c}^{\prime}\right)$ & $17 \mathrm{keV}$ & $<5.7 \mathrm{keV}$ \\
\hline$B_{\gamma}\left(\psi^{\prime}+n_{c}^{\prime}\right)$ & $7.5 \%$ & $<2.5 \%$ \\
\hline $\mathrm{B}_{\gamma}\left(\psi^{\prime} \rightarrow \eta_{c}^{\prime}\right) B\left(\eta_{c}^{\prime} \rightarrow \gamma \gamma\right)$ & $1.0 \times 10^{-2} \%$ & $<3.1 \times 10^{-2} \%$ \\
\hline$\Gamma_{\gamma}\left(n_{c}^{r} \rightarrow \psi\right)$ & $0.53 \mathrm{keV}$ & -- \\
\hline${ }_{\gamma}^{B}\left(n_{c}^{\prime} \rightarrow \psi\right)$ & $1.6 \times 10^{-2 \%}$ & $>24 \pm 16 \%$ \\
\hline$B_{\gamma}\left(\psi^{\prime} \rightarrow n_{c}^{\prime}\right) B_{\gamma}\left(n_{c}^{\prime} \rightarrow \psi\right)$ & $1.2 \times 10^{-3} \%$ & $0.6 \pm 0.4 \%$ \\
\hline
\end{tabular}


$\psi \rightarrow \gamma \mathrm{n}_{\mathrm{c}}$. This is espectally puzzling when one recalls that M1 transitions among the light mesons are fairly well-described by the nonrelativistic quark model $(72,76)$. Particularly relevant to $\psi \rightarrow \gamma \eta_{c}$ are the predictions $\Gamma(\phi \rightarrow \gamma \pi)=70 \mathrm{keV}$ and $\Gamma\left(\phi \rightarrow \gamma \pi^{\circ}\right)=6.9 \mathrm{keV}$, to be compared to the measured widths of $82 \pm 16 \mathrm{keV}$ and $5.7 \pm 2.0 \mathrm{keV}$, respectively. To add to the puzzle, there is the apparently successful prediction of a strongly suppressed $\psi^{\prime \prime} \rightarrow \gamma{ }_{c}$ transition.

(3) While the prediction $B\left(\psi^{\prime} \rightarrow \gamma n_{c}^{\prime}\right)=0.075$ i.s only three times larger than the observed branching ratio limit, the inferred lower limit,

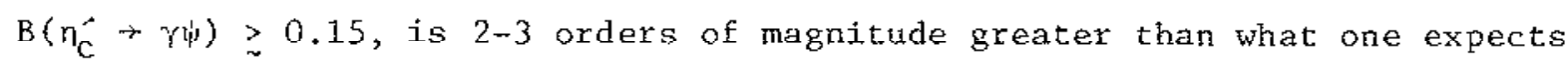
theoretically for this hindered MI transition.

If $\mathrm{x}(2830)$ really is the $\eta_{c}$, the resolution to these difficulties must lie partly in correcting the assumption of identical radial wave functions for $\psi$ and $n_{e}$, i.e. that gluons play an important role in suppressing both the Ml overlap integral and $\Gamma\left(\eta_{c} \rightarrow 2\right.$ gluons $) / \Gamma\left(n_{c} \rightarrow \gamma \gamma\right)$. On the other hand, given the successes of gluon counting for direct decays of spin-triplet states, the verified suppression of the hindered M] transition $\psi^{-} \rightarrow \gamma_{c}$, and the experimental fact that $\Gamma\left(\psi \rightarrow \gamma \eta_{c}\right) \leqslant 1 \mathrm{keV}$, there is no way to understand the identification $x(3455)=n_{c}^{-}$with such a large branching ratio to $\gamma \psi$.

It is always possible, of course, that $\eta_{c}$ and $\eta_{c}^{\prime}$ have not been discovered yet and that they lie $\leq 100 \mathrm{MeV}$ below their hyperfine partners, as originally expected $(47,53)$. In that case, theoretical estimates of the $\mathrm{Ml}$ rates are greatly reduced and, in fact, lie within experimental limits for states at such masses.

The natural question then is: What are these two states? Various conjectures

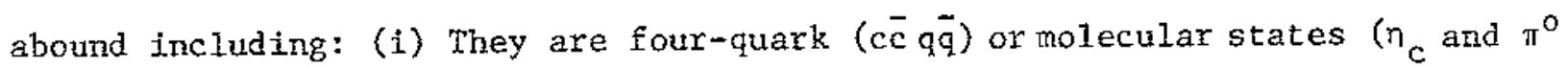
bound in an $\mathrm{S}$ wave, say) (77) or, perhaps $\mathrm{c} \overline{\mathrm{c}}$ states with a gluon excitation (78) There are no convincing models for such relatively low-mass systems which are not pure cic, much less the ability to make convincing estimates of transition 
rates. Such techniques are sorely needed.

(ii) Another interesting speculation is that they are Higgs mesons $\mathrm{H}$

(79). If this is the case, $H$ certainly does not have the "conventional"

coupling to quarks $\sim G^{\frac{1}{2}} F_{q}^{m}$, for then one would estimate (with $\mathrm{m}_{\mathrm{C}} \simeq \mathrm{M}_{\psi, \psi} / 2$ )

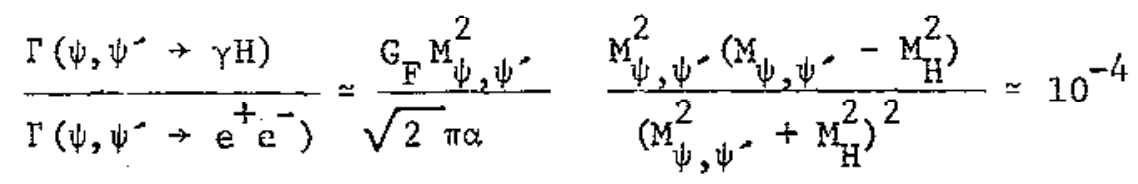

Not only does one need the coupling of $\mathrm{H}$ to charmed quarks to be anomalously large, but the coupling to light quarks must be anomalously small if $H \rightarrow \gamma \gamma$ is to be a sizable decay mode $(80)$. (Actually, $X(3455)=H \rightarrow \psi \gamma$ might be a dominant decay mode of a more-or-less conventional Higgs meson.)

(iii) One final possibility, suggested by Harari (81), is that $x(3455)$ is the ${ }^{D_{2}}$ level of charmonium. Because of the strongly hindered nature of ${ }^{3} \mathrm{~S}_{1} \rightarrow{ }^{1} \mathrm{D}_{2}$ radiative transitions, such a possibility is viable only if $\psi^{\prime}$ and $\psi$ contain a sizable admixture $(\sim 5-10 \%)$ of ${ }^{3} D_{1}$ and if $\Gamma\left({ }^{1} D_{2} \rightarrow\right.$ hadrons $) \simeq \Gamma(\psi \rightarrow$ hadrons) (82). Perhaps the most serious objection to this identification, based as it is on the presumed large splitting between all triplet and singlet states of given $L$, is that $\Gamma\left(\psi^{-} \rightarrow \gamma{\pi_{c}^{\prime}}_{c}\right)$ is expected to be $v 10 \Gamma\left(\psi^{-} \rightarrow \gamma^{1} D_{2}\right)$ and yet, on this hypothesis, $n_{c}^{\prime}$ has not been seen yet.

To sumarize: While the basic model gives a very good qualitative, and creditable quantitative, description of the spectrum and transition rates of the spin-triplet charmonium levels, it fails to account for practically all observed features of the proposed singlet levels. Either something very important (and largely unknown) is missing from the model or, more happily, the model is telling us that new degrees of freedom which it was never intended to handle-have been discovered. 


\section{INCLUDING SPIN DEPENDENCE}

Even before the states between $\psi$ and $\psi^{*}$ were discovered and their splittings measured, many people began trying to incorporate quark spin dependence into the charmonium model. The very earliest work utilizing a coulomb model $(47,53,69)$ was tach too naive and soon abandoned. However, the nonrelativistic character of the low-lying cc states suggested that the Bethe-Salpeter equation would be a useful formalism and that some analog of the Breit-Fermi Hamiltonian for positronium would continue to be relevant. Almost nothing was known about the structure of the Bethe-Salpeter kernel and some educated guesses were needed to make the computation of splittings possible. Some perturbation- theoretic analyses of QCD already suggested that the spin-dependent part of the Hamiltonian would be strongly modified away from a Coulomb form just as the spin-independent confining part is (83). These same investigations further indicated that the modifications of the two pieces might not be simply related.

One early guess, however, was that the spin-dependent interaction would have only a short-range Coulomb-type structure (84). Very small fine structure splittings were predicted, such as $M_{\psi}-M_{n_{C}} \simeq 30 \mathrm{MeV}$ and $M\left({ }^{3} P_{2}\right)-M\left(P_{1}\right) \simeq 5-10$ MeV. This is in sharp disagreement with experiment, and such an approach now seets inadequate. In particular, Johnson (85) has recently emphasized that at least one part of the spin effect is necessarily long range, namely that part of the spin-orbit interaction arising from Thomas precession (see Eq. (3.37) below). Indeed, almost all treatments of the spin forces in charmonium have focussed on the long-range part of the c $\bar{c}$ interaction. One exception is the model of Celmaster et al (86), mentioned below in Sec. III.F. They assume an $r$-dependent short-distance coupling $k(r)$, whose form is suggested by asymptotic freedom, and use $V_{A}, F$. $(r)=-k(r) / r$ to generate the Breit-Fermi interaction. Although they obtained much larger splittings than in Ref. 84 (see Table 6). 
they have neglected to take account of the long-range contribution from Thomas precession.

The most popular approach, pioneered by Schnitzer (87) and by Pumplin, Repko, and Sato (88), and since generalized by many authors (89), has been to assume that heavy quark binding is effectively due to "single gluon exchange with renormalization group improvement", sumarized by an instantaneous Bethe-Salpeter kernel consisting of vector and scalar interaction terms:

$$
v_{\text {coul } 1 .}\left(\vec{k}^{2}\right) \gamma_{1}^{\mu} \gamma_{2 \mu}+v_{v}\left(\vec{k}^{2}\right) \Gamma_{1}^{\mu}(k) \Gamma_{2 \mu}(k)+v_{s}\left(\vec{k}^{2}\right) \mathbb{1}_{1} \mathbb{1}_{2}
$$

The subscripts 1 and 2 refer to the $c$ and $\bar{c}$ quarks, $\mathbb{1}$ is a unit natrix in Dirac space, $k$ is the 4-momentum carried by the exchanged gluon, and (with

$$
\begin{aligned}
& \left.\sigma_{\mu \nu}=\frac{1}{2 i}\left[\gamma_{\mu}, \gamma_{\nu}\right]\right) \\
& \quad \Gamma_{\mu}(k)=\gamma_{\mu}-\frac{i \lambda}{2 m_{c}} \sigma_{\mu \nu} k^{\nu}
\end{aligned}
$$

where $\lambda$ is the color magnetic moment of the quarkan adjustable parameter. In the spin-independent, nonrelativistic limit of this interaction, the potential is

$$
v_{0}=v_{\text {cou } 1}+v_{v}+v_{s} \text {. }
$$

In most discussions, it has been assumed that

$$
\mathrm{v}_{\mathrm{v}}=n \mathrm{v}_{1 \mathrm{in}} \quad \mathrm{v}_{\mathrm{s}}=(1-n) \mathrm{v}_{1 \text { in }}
$$

where $V_{l i n}$ is the linear confining potential, $r / a^{2}$ in coordinate space, and $n$ is another adjustable parameter.

Having made the ansatz, Eqs. (3.33)-(3.36), these authors then obtained the spin-dependent potential by following the same steps by which one converts the 
kernel for positronium into the Breit interaction. The result is:

$$
\begin{aligned}
& -V_{\operatorname{spin}}(r)=\frac{1}{2 m_{c}^{2}}\left[\frac{4 k}{r^{3}}+4(1+\lambda) \frac{1}{r} \frac{d V_{v}}{d r}-\frac{1}{r} \frac{d V_{o}}{d r}\right] \vec{L} \cdot \vec{S} \\
& +\frac{2}{3 m_{c}^{2}}\left[4 \pi k \delta(\vec{r})+(1+\lambda)^{2} \nabla^{2} v_{v}(r)\right] \vec{S}_{1} \cdot \vec{S}_{2} \\
& +\frac{1}{3 m_{c}^{2}}\left[\frac{3 k}{r^{3}}+\frac{1}{r} \frac{d v}{d r}-\frac{d^{2} v}{d r^{2}}\right] S_{12}
\end{aligned}
$$

where

$$
s_{12}=3 \vec{s}_{1} \cdot \hat{r} \vec{s}_{2} \cdot \hat{r}-\vec{s}_{1} \cdot \vec{S}_{2}
$$

The last term in the spin-orbit part of $\mathrm{V}_{\text {spin }}$ is the Thomas precession contribution, and contains the only influence of the scalar interaction on spin-dependence. For the potentials in Eq. (3.36), $\mathrm{V}_{\text {spin }}$ is given by

$$
\begin{aligned}
V_{\text {spin }}(r)= & \frac{1}{2 m_{c}^{2}}\left[\frac{3 \kappa}{x^{3}}+\frac{1}{r^{2}}(n(3+4 \lambda)-(1-n))\right] \overrightarrow{\mathrm{L}} \cdot \overrightarrow{\mathrm{S}} \\
& +\frac{2}{3 \mathrm{~m}_{\mathrm{c}}^{2}}\left[4 \pi k \delta(\overrightarrow{\mathrm{r}})+\frac{2 \mathrm{n}}{\mathrm{ra}^{2}}(1+\lambda)^{2}\right] \overrightarrow{\mathrm{S}}_{I} \cdot \overrightarrow{\mathrm{s}}_{2} \\
& +\frac{1}{3 \mathrm{~m}_{\mathrm{c}}^{2}}\left[\frac{3 k}{\mathrm{r}^{3}}+\frac{\mathrm{n}}{\mathrm{ra}^{2}}(1+\lambda)^{2}\right] \mathrm{S}_{12} .
\end{aligned}
$$

When used perturbatively, this interaction generates the following mass formulae (below, $M_{L}=$ bare mass of the level $\psi_{n L}$ determined by $v_{0}$, and $\left\langle\mathrm{x}^{-\mathrm{c}}\right\rangle_{\mathrm{L}}=\left\langle\psi_{\mathrm{nL}}\left|\mathrm{r}^{-\mathrm{c}}\right|_{\psi_{\mathrm{nL}}}\right\rangle:$ 


$$
\begin{aligned}
& M\left({ }^{3} S_{1}\right)=M_{0}+\frac{1}{6 m_{c}^{2}}\left[4 \pi k\left|\Psi_{n 0}(0)\right|^{2}+2(1+\lambda)^{2} n^{-2}\left\langle r^{-1}\right\rangle_{0}\right] \\
& M\left({ }^{1} S_{0}\right)=M_{0}-\frac{1}{2 m_{c}^{2}}\left[4 \pi k\left|\Psi_{n 0}(0)\right|^{2}+2(1+\lambda)^{2} n^{a}{ }^{-2}\left\langle r^{-1}\right\rangle_{0}\right] \\
& M\left({ }^{3} P_{2}\right)=M_{1}+\frac{1}{5 m_{c}^{2}}\left\{7 \kappa \cdot\left\langle r^{-3}\right\rangle_{1}+\left[\left(9+13 \lambda+\frac{3}{2} \lambda^{2}\right) n-\frac{5}{2}(1-n)\right] a^{-2}\left\langle r^{-1}\right\rangle\right\} \\
& M\left({ }^{3} P_{1}\right)=M_{1}+\frac{1}{2}\left\{-k\left\langle r^{-3}\right\rangle_{1}+\left[\left(-1-\lambda+\frac{1}{2} \lambda^{2}\right) n+\frac{1}{2}(1-n)\right] a^{-2}\left\langle r^{-1}\right\rangle_{1}\right\} \\
& M\left({ }^{3} P_{0}\right)=M_{1}-\frac{1}{m_{c}^{2}}\left\{4 \kappa\left\langle r^{-3}\right\rangle_{1}+[(3+4 \lambda) n-(1-n)] a^{-2}\left\langle r^{-1}\right\rangle_{1}\right\} \\
& M\left({ }^{1} P_{1}\right)=M_{1}-\frac{(1+\lambda)^{2} n}{m_{c}{ }^{2}}\left\langle r^{-1}\right\rangle_{1}
\end{aligned}
$$

For completeness, we include formulae for the splitting of D-levels and mixing of ${ }^{3} \mathrm{D}_{1}$ with ${ }^{3} \mathrm{~S}_{1}$ :

$$
\begin{aligned}
& M\left({ }^{3} D_{3}\right)=M_{2}+\frac{1}{7 \pi n_{c}^{2}}\left\{2 0 k \left\langler^{-3} / 2+\left[\left(23+32 \lambda+2 \lambda^{2}\right) \eta-7(1-n)\right] \cdot a^{-2}\left\langle r^{-1}\right\rangle\right.\right. \\
& M\left({ }^{3} D_{2}\right)=M_{2}+\frac{1}{m_{c}^{2}}\left\{-k\left\langle r^{-3}\right\rangle_{2}+\left[\left(-1-\lambda+\frac{1}{2} \lambda^{2}\right) n+\frac{1}{2}(1-n)\right] a^{-2}\left\langle r^{-1}\right\rangle_{2}\right\} \\
& M\left({ }^{3} D_{1}\right)=M_{2}+\frac{1}{6 m_{c}^{2}}\left\{-30 \kappa\left\langle r^{-3}\right\rangle_{2}+\left[\left(-26-34 \lambda+\lambda^{2}\right) n+9(1-n)\right] a^{-2}\left\langle r^{-1}\right\rangle_{2}\right\} \\
& M\left({ }^{1} D_{2}\right)=M_{2}-\frac{(1+\lambda)^{2} n}{m_{c}^{2}{ }^{2}}\left\langle x^{-1}\right\rangle_{2} \\
& \left.\left\langle\mathrm{n}^{3} \mathrm{~S}_{1}\left|\mathrm{v}_{\mathrm{spin}}\right| \mathrm{m}^{3} \mathrm{D}_{1}\right\rangle=\frac{\sqrt{8}}{12 \mathrm{rn}_{\mathrm{C}}^{2}}\left[3 \times k \hat{\mathrm{NS}}\left|\mathrm{r}^{-3}\right| \mathrm{mD}\right\rangle+(1+\lambda)^{2} \mathrm{na}^{-2}\left\langle\mathrm{nS}\left|\mathrm{r}^{-1}\right| \mathrm{mD}\right\rangle\right] .
\end{aligned}
$$

The splittings atnong the $P$ and $S$ states determined by various authors Erom Eq. (3.39) are 1isted in Table 6, together with the measured mass 
Table 6

Spin-dependent splittings in charmonium. The numbers in brackets are values of $\mathrm{k}, 1 / \mathrm{a}^{2}\left(\mathrm{GeV}^{2}\right), \mathrm{m}_{\mathrm{c}}(\mathrm{GeV}), \eta$ and $\lambda$ used by various authors. In Ref. 86 the Coulomb parameter is not constant (see Eq. 3.70) and only this short-range part of the potential is kept in computations of spin-dependence.

\begin{tabular}{|c|c|c|c|c|}
\hline Author; Parameters & $\mathrm{M}_{\mathrm{X}_{2}}-\mathrm{M}_{\mathrm{X}_{1}}$ & ${ }^{M} x_{1}-M_{x}$ & $M_{\psi}-M_{n_{c}}$ & $M_{\psi}-M_{n}$ \\
\hline Experiment & $44 \pm 6$ & $95 \pm 5$ & $265 \pm 14$ & $230 \pm 7$ \\
\hline $\begin{array}{l}\text { Schnitzer (Ref. } 87) \\
{[0.2,0.19,1.6,1.0,0.0]}\end{array}$ & 87 & 63 & 70 & 58 \\
\hline $\begin{array}{l}\text { Pump1in, et a1 (Ref. 88) } \\
{[0.0,0.30,1.5,1.0,0.0]}\end{array}$ & 152 & 117 & 119 & 92 \\
\hline $\begin{array}{l}\text { Henriques, et a1 (Ref. } 89 \text { ) } \\
{[0.8,0.18,1.6,0.0,0.0]}\end{array}$ & 40 (input) & 80 & 95 & -- \\
\hline $\begin{array}{l}\text { Schnitzer (Ref. } 87) \\
{[0.2,0.19,1.6,1.0,1.1]}\end{array}$ & 182 & 170 & 268 (input) & 225 \\
\hline $\begin{array}{l}\text { Chan (Ref. 89) } \\
{[0.2,0.15,1.6,0.12,5.0]}\end{array}$ & 40 (input) & 90 & 262 (input) & 225 \\
\hline $\begin{array}{l}\text { Carlson and Gross (Ref. 89) } \\
{[0.27,0.20,1.37,0.08,4.4]}\end{array}$ & 41 (input) & 98 & 265 (input) & 181 \\
\hline $\begin{array}{l}\text { Celmaster, et al (Ref. } 86 \text { ) } \\
{[-,-, 1.98,1.0,0.0]}\end{array}$ & 92 & 100 & 150 & 80 \\
\hline
\end{tabular}


differences; for comparison purposes, we assumed $x(2830)=1^{1} \mathrm{~S}_{0}$ and $\times(3455)=2^{1} \mathrm{~S}_{0}$. For the D states we content ourselves with two remarks. First the $I^{3} D_{1}$ level comes out about $40 \mathrm{MeV}$ below its unperturbed mass ${ }^{8}$ (which is $3.755 \mathrm{GeV}$ in Table 2). Thus, the agreement between the coupled-channel calculation (in Sec. III.E) and the observed mass $3.772 \mathrm{GeV}$ of $\psi^{\text {"i }}$ is perhaps fortuitous. Second, the predicted mixing angle between $1^{3} \mathrm{D}_{1}$ and $2^{3} \mathrm{~s}_{1}$, is

$$
\varepsilon=\frac{1}{2} \tan ^{-1} \frac{2\left\langle 2^{3} s_{1}\left|v_{\text {spin }}\right| 1^{3} D_{1}\right\rangle}{M\left(2^{3} D_{1}\right)-M\left(2^{3} s_{1}\right)} \simeq 6^{\circ}
$$

This is much less than one infers from measurements of the $\psi^{\prime \prime}$ electronic width. Using

$$
\varepsilon=\tan ^{-1}\left\{\frac{M_{\psi^{\prime \prime}}}{M_{\psi^{*}}}\left[\frac{\Gamma\left(\psi^{\prime \prime} \rightarrow \mathrm{e}^{+} \mathrm{e}^{-}\right)}{\Gamma\left(\psi \rightarrow \mathrm{e}^{+} \mathrm{e}^{-}\right)}\right]^{1 / 2}\right\},
$$

these are $\varepsilon=(26 \pm 3)^{\circ}$ for $\mathrm{I}_{\mathrm{e}}\left(\psi^{\prime \prime}\right)=0.37 \pm 0.09 \mathrm{keV}(90)$, and $\varepsilon=(19 \pm 3)^{\circ}$ for $T_{e}\left(\psi^{\prime \prime}\right)=0.18 \pm 0.05 \mathrm{keV} \cdot(91)$.

The lessons of this attack on the problem of spin-dependence may be summarized thus: Insofar as one is willing to extend the hypothesis of an instantaneous interaction between heavy quarks beyond the realm of the simple spin-independent potential $\mathrm{V}_{\mathrm{o}}$, the ansatz Eq. (3.33) is the basis of a quite reasonable first effort. Clearly, the assumption of a purely short-range origin for spin forces is inadequate. Economy of parameters then demands that one attribute spin forces to the long-range part, here assumed to be $\mathrm{r} / \mathrm{a}^{2}$. As we have seen, this still leaves some freedon in the Dirac structure of the kernel, and the work of Henriques et al (89) first suggested that the P-state splittings are best fit if the long-range interaction is scalar. The reason for this is that the Thomas precession term, which is the most important part of the spin-orbit interaction in this case ( $n$ smail), orders the ${ }^{3} \mathrm{P}_{J}$ ievels oppositely from the other terms in $\mathrm{V}_{\text {spin }}$. This feature is needed to explain the 
unexpectedly small ratio $\left(M\left({ }^{3} P_{2}\right)-M\left({ }^{3} P_{I}\right)\right) /\left(M\left({ }^{3} P_{1}\right)-M\left({ }^{3} P_{0}\right)\right)=0.42$.

If one now assigns $x(2830)$ to $\eta_{c}\left(1^{I} s_{0}\right)$, the splitting from $\psi$ may be fit, as Schnitzer found (87), by assuming a rather large quark color anomalous moment, $\lambda(n)^{1 / 2} \simeq 1$. A11 this was put together by Chan and by Carlson and Gross (89) who combined the successful features of the last. two named pieces of work to obtain excellent agreement with the data.

But, In this flush of success, one must not lose sight of two important facts. The first is that all that has been accomplished so far is to fit four mass splittings with two parameters, $\lambda$ and $\eta$. In fact, as Carlson and Gross point out, $M\left({ }^{3} P_{1}\right)-M\left({ }^{3} P_{0}\right)$ is far less sensitive to parameters than is $M\left({ }^{3} P_{2}\right)-$ $M\left({ }^{3} \mathrm{P}_{1}\right)$. Therefore, a fit to the latter plus the $\psi-\mathrm{X}(2830)$ splitting almost automatically gets the former right. And, it is not surprising that a large $1^{3} s_{1}-1^{1} s_{0}$ implies a comparable $2^{3} s_{1}-2^{1} s_{0}$ splitting. The real test of this phenomenology will come when the multitude of intramultiplet splittings in the $T$ - system are measured--a potentialiy difficult task if they are $\sim 5-10$ times smaller, as expected. Secondly, given the difficulty of accommodating the $X(2830)$ and $X(3455)$ in the simple charmonium scheme, it may well be that it has been a great mistake all along to use these states in determining the parameters in $\mathrm{V}_{\text {spin }}$. Again, only time will tell.

From a theoretical standpoint, the procedure outlined above suffers from the lack of a "first principles" justification for its starting point, the BetheSalpeter kernel defined in Eqs. (3.33) and (3.36). More ambitious approaches are in progress in a number of dynamical models which incorporate gluon degrees of freedom more or less explicitiy. These include lattice gauge theories (51, 52) the MIT bag model $(92,85)$, and the quark-string models $(78,93)$. In each of these models, the long-range spin-independent potential between heavy quarks is shown to be linear. Further there is a reasonably well-defined procedure for 
extracting the spin-dependent interaction. While it is too early to assess these approaches, it is hoped that they will provide insight to this most puzzling aspect of charmonium dynamics.

\section{E. COUPLING CHARMONIUM TO TTS DECAY CHANNELS}

It was recognized very early (63) that the quantum mechanical coupling between charmonium states and their OZI-allowed decay channels could modify the predictions of the oaive potential model. The development of a model for this coupling and the resulting predictions have been presented in a series of papers by Eichten et al $(75,94,68,95) .{ }^{9}$ The main issues one wants to address in such a mode]. are these:

1. Renormalization (shifts) of the bare spectrum generated by $V_{0}(r$ ) and the widths of $\bar{c} \bar{c}$ states above charm threshold, $W_{c}=2 M_{D^{\circ}}=3.727 \mathrm{GeV}$.

2. Renormalizations of the wave functions deduced from $\mathrm{V}_{0} .^{*}$ This includes leakage from the $c \bar{c}$ sector to the charmed hadron sector, as well as the mixing among charmonium levels having the same $J$ PC. Both these will affect rates for all the transitions discussed in Sec. III.C. Of special interest is the fact that decays which are forbidden or strongly suppressed in the potential model, such as ${ }^{3} \mathrm{D}_{1} \rightarrow \mathrm{e}^{+} \mathrm{e}^{-}$and $2^{3} \mathrm{~s}_{1} \rightarrow 1^{1} \mathrm{~s}_{0}+\gamma$, can be enhanced through mixing between $1^{3} \mathrm{D}_{1}$ and $2^{3} \mathrm{~S}_{1}$, and between $2^{3} \mathrm{~s}_{1}$ and $1^{3} s_{1}$ (or $2^{1} s_{0}$ and $1^{1} s_{0}$ ), respectively.

3. A description of $\mathrm{e}^{+} \mathrm{e}^{-}$annihilation in the charm threshold region, $W_{c} \leq W \lesssim 4.4 \mathrm{GeV}$. In particular, one wants to interpret the structure of $R=\sigma\left(\mathrm{e}^{+} \mathrm{e}^{-}+\right.$hadrons $) / \sigma\left(\mathrm{e}^{+} \mathrm{e}^{-} \rightarrow \mu^{+} \mu^{-}\right)$in this region, and to discuss the general (and sometimes peculiar) features of the exclusive channel cross-sections $\sigma\left(e^{+} e^{-} \rightarrow D^{\circ} \bar{D}^{\circ}\right), \sigma\left(e^{+} e^{-} \rightarrow D^{+} D^{-}\right), \sigma\left(e^{+} e^{-} \rightarrow D^{\circ} \bar{D}^{* 0}\right)$, etc. 
FORMALISM AND DESCRIPTION OF THE MODEL The description of what happens to a discrete set of states in one part of Hilbert space when it is immersed in and coupled to a continuun of states belonging to another subspace is a classic problem in quantum mechanics and was, formally, solved long ago (96).

Let the total Hamiltonian of this system be

$$
H=H_{0}+H_{I}
$$

where $H_{0}$ is responsible for the binding of the discrete states $|n\rangle$ and the continuum states $|v\rangle$ :

$$
\begin{array}{ll}
H_{0}|n\rangle=\varepsilon_{n}|n\rangle, & \langle n \mid m\rangle=\delta_{n m}, \\
H_{0}|v\rangle=\omega_{v}|v\rangle, & \langle v \mid \mu\rangle=\delta(v-\mu), \\
\langle v \mid n\rangle=0 &
\end{array}
$$

while $H_{I}$ is responsible for their coupling:

$$
\langle\nu|\mathrm{H}| \mathrm{n}\rangle=\left\langle\nu\left|\mathrm{H}_{I}\right| \mathrm{n}\right\rangle
$$

In the present short-hand notation, $|n\rangle$ stands for any of the pure $c \bar{c}$ levels $\left|\psi_{n L J}\right\rangle$, and $|v\rangle$ for any state of charmed hadrons having zero net charm, zero total momentum, and total energy $\omega_{v} \cdot$ (States with more than one $c$ and one $\bar{c}$ axe ignored.)

The problem we face is to describe the eigenstates $|N\rangle$ of $H$ with eigenvalue $\mathrm{E}_{\mathrm{N}}$. These states include the observed bound states $\left(\psi, \chi_{J}, \psi^{\prime}\right.$, etc.) below charmed threshold as well as the continum states and resonances in $\mathrm{e}^{+} \mathrm{e}^{-}$ annihilation above $\mathrm{W}_{\mathrm{C}}$. (Because $\mathrm{H}$ allows for decay, $\mathrm{E}_{\mathrm{N}}$ need not be real-and certainly won't be for the resonances). We begin by expanding $\mid N$, in the complete basis formed by the eigenstates $|n\rangle$ and $|v\rangle$ of $\mathrm{H}_{\mathrm{O}}$ : 


$$
\begin{array}{ll}
|N\rangle=\sum_{n} a_{n N}|n\rangle+\int d \nu a_{v N}|\nu\rangle \\
a_{n N}=\langle n \mid N\rangle, & a_{v N}=\langle v \mid N\rangle
\end{array}
$$

From Eq. (3.44)-(3.46), the expansion coefficients and the energy $E_{N}$ are solutions of the eigenvalue problem

$$
\begin{aligned}
& \sum_{\mathrm{m}}\left[\left(E_{\mathrm{N}}-\varepsilon_{\mathrm{n}}\right) \delta_{\mathrm{nm}}-\Omega_{\mathrm{nm}}\left(E_{\mathrm{N}}\right)\right] \mathrm{a}_{\mathrm{mN}}=0 \\
& \operatorname{det}[W-\varepsilon-\Omega(W)]=0 \quad\left(\operatorname{Roots} E_{\mathrm{N}}\right)
\end{aligned}
$$

where

$$
\begin{aligned}
\Omega_{\mathrm{nm}}(W) & =\int \frac{d v}{W-\omega_{v}+\frac{10}{0}}\left\langle\mathrm{n}\left|\mathrm{H}_{I}\right| v\right\rangle\left\langle v\left|\mathrm{H}_{I}\right| \mathrm{m}\right\rangle \\
& \equiv \Delta_{\mathrm{nm}}(W)-\frac{i}{2} \Gamma_{n m}(W) .
\end{aligned}
$$

For $E_{N}<W_{c}$, the solutions of Eqs. $(3.47)$ and $(3.48)$ correspond to the bound charmonium levels, the matrix $\Delta_{\mathrm{nm}}\left(\mathrm{E}_{\mathrm{N}}\right)$ describes the shift in the mass of these levels from the "bare" masses $\varepsilon_{\mathrm{n}}$, and the width matrix $\Gamma_{\mathrm{nm}}\left(\mathrm{E}_{\mathrm{N}}<W_{\mathrm{C}}\right)$ vanishes. For $\left|E_{N}\right|>W_{C}$, the state $|N\rangle$ is a resonance which decays almost exclusively to charned hadrons, having mass $M_{N}$, width $\Gamma_{N}$, and $E_{N}=M_{N}-\frac{i}{2} \Gamma_{N}$. Given the coefficients $a_{n N}$, one may determine $a_{U N}$ from

$$
a_{\nu N}=\frac{1}{E_{N}-\omega_{\nu}+i 0} \sum_{m} a_{m N}\left\langle v\left|H_{I}\right| m\right\rangle
$$

Finally, the continuum eigenstates $|N\rangle$ may be determined from integral equations similar in structure to Eqs. (3.47) and (3.48). For reasons that will. become clear shortly, the model makes little use of these. Rather, the recalculation of the transition rates of the bound states and of

$$
\Delta R \equiv \frac{\sigma\left(\mathrm{e}^{+} \mathrm{e}^{-}+\text {charmed hadrons }\right)}{\sigma\left(\mathrm{e}^{+} \mathrm{e}^{-} \rightarrow \mu^{+} \mu^{-}\right)}
$$


requires only a knowledge of $\Omega$ which, in turn, requires a model for $\mathrm{H}_{\mathrm{I}}$.

The assumptions and approximations defining the model for $\mathrm{H}_{\mathrm{I}}$ and $\Omega$ are $(68,75)$ :

1. The Hamiltonian is taken to be

$$
H=-\frac{3}{8} \sum_{a=1}^{8} \int d^{3} x d^{3} y \rho_{a}(\vec{x}, t) v(\vec{x}-\vec{y}) \rho_{a}(\vec{y}, t)
$$

+ quark kinetic energy terms

where

$$
\rho_{a}(x)=\sum_{\text {flavors }(i)} q_{i}^{\dagger}(x) \frac{\lambda}{2} q_{i}(x) \text { is the octet of color charge }
$$

densities and $v(\vec{x}-\vec{y})=|\vec{x}-\vec{y}| / a^{2}$ is the instantaneous confining potential in

Eq. (3.8). The Coulomb piece has been dropped for simplicity. 10

2. As the form of $V$ implies, calculations with this Hamiltonian necessarily are carried out in the nonrelativistic approximation. Therefore, $\mathrm{H}$ is explicitly spin-independent. The only dependence on quark total spin that enters the computation of $\Omega$ is through the use of "spin-split thresholds" for the continum states $|\nu\rangle$, i.e. the mass difference between $D^{*}$ and $D, F^{*}$ and $F$ is put in by hand.

3. When decomposed into creation and annihilation operators, $\mathrm{H}=\mathrm{H}_{\mathrm{O}}$ (binding of $\left.q_{i} \bar{q}_{j}\right)+H_{I}$ (pair emission: $q_{i}+q_{i}+\bar{q}_{j}+q_{j}$ ) + other terms (e.g., emission of two pairs from the vacuum) which are discarded. While the nonrelativistic binding mechanistn is presumably consistent only for $c \bar{c}$ states (where it reproduces the results of the spin-independent potential model described in Sec. IV.C), it is also used to generate bound-state wave functions for charmed meson states $c \bar{q}$ and $\bar{c} q(q=u, d, s$ from now on).

4. The model assumes that the transition $\psi_{\mathrm{nLJ}} \rightarrow$ charmed mesons is a sequential quasi-two-body process, ${ }^{11}$ e.g., $\psi_{n L J}+\overline{b D} * D^{*} \rightarrow D \pi$. Accordingly, the only terms kept in $\mathrm{H}_{\mathrm{I}}$ are those describing light-pair emission, $c \rightarrow c+q+\bar{q}$ and $\bar{c} \rightarrow \bar{c}+q+\bar{q}$, which govern $\psi_{n L J}(\bar{c} \bar{c})+D(c \bar{q})+\bar{D}(\bar{c} q)$, as depicted in Fig. 5 . 
There, the shaded circles denote bound-state vertex functions (simply related to the wave functions in the nonrelativistic limit) and they emphasize that the model incorporates the extended nature of the parent and its decay products. While certain features (to be mentioned below) of the transition amplitudes computed with $\mathrm{H}_{\mathrm{I}}$ may be model-independent, the nonrelativistic approximation used in the computation is very questionable.

5. The final approximation made in Refs. 68,75 is a drastic truncation of the continuum states $|v\rangle$ to include only the ground state charmed mesons $\mathrm{D}, \mathrm{D}^{*}$, $F$, and $F^{*}$. ConsequentIy, the model is reliable (even semiquantitatively) only where the effects of higher thresholds (e.g. charmed P-states) may be ignored. For the calculation of $\Delta R$, the breakdown due to neglect of higher thresholds is already apparent at $\mathrm{W} \approx 4.1 \mathrm{GeV}$.

The general form of the transition amplitude for $\psi_{n L J} \rightarrow$ pair of ground state charmed mesons is

$$
\begin{aligned}
& \left\langle\overline{\operatorname{cq}}\left(J_{1} ; E_{1}, \vec{p}\right), \overline{c q}\left(J_{2} ; E_{2},-\vec{p}\right)\left|H_{I}\right| \overline{c c}(n I, ; W, \overrightarrow{0})\right\rangle \\
& =\left(m \cdot \frac{m_{c} m^{m}}{m_{c}+m_{q}}\right)^{-1} \times \operatorname{Spin} \text { Factor }\left(J_{i}\right) \times \text { Form Factor }\left(n, L ;|\vec{p}| ; m_{c}, m_{q}, a\right)
\end{aligned}
$$

where $\left(E_{i}, \vec{p}_{i}\right)$ are the 4-momenta of the outgoing pair, $W=E_{1}+E_{2}$, and $J_{i}$ are total angular momenta. The parameters entering the calculation of this amplitude are the quark masses $m_{c}, m_{q}$ and the linear potential strength $a^{-2}$. Because of the spin-independence of the $q \bar{q}$ production mechanism, all dependence on quark spin appears in a trivial Clebsch, the second factor in Eq. (3.53). The first factor, which implies a suppression of transition to $F=c \bar{s}$ relative to $\mathrm{D}=\mathrm{cu}$, arises from the $\mathrm{S}$-wave nature of the production mechanism and from the charmed meson wave function. The P-wave form factors for the first three 
$L=0 \mathrm{c} \bar{c}$ levels to go to a $\bar{D} \bar{D}$ pair are plotted in Fig. 6 as a function of the D momentum, $|\vec{p}|$. The high-momentum fall-off and the oscillations reflect the finite extent of the bound states and the nodes in the wave functions of radial excitations. These zeroes in the form factor play an important role in the behavior of the exclusive cross-sections $\sigma\left(\mathrm{e}^{+} \mathrm{e}^{-} \rightarrow \mathrm{D} \overline{\mathrm{D}}, \mathrm{DD}^{-{ }^{*}}, \mathrm{D}^{*} \overrightarrow{\mathrm{D}}^{*}\right)$ as a function of center-of-mass energy $W$.

The calculation of level positions, radiative transition rates, and $\Delta R$ in this model proceeds as follows:

1. RENORMALIZATION OF SPECTRUM The parameters of the model are $\mathrm{m}_{c}$ and a, the Iight-quark masses $\mathrm{m}_{\mathrm{u}}=\mathrm{m}_{\mathrm{d}}$ and $\mathrm{m}_{\mathrm{S}}$, and the charmed meson masses $\mathrm{M}_{\mathrm{D}}, \mathrm{M}_{\mathrm{D}} *, \mathrm{M}_{\mathrm{F}}$, and $\mathrm{M}_{\mathrm{F}^{*}}$. The last named are now chosen to have their measured values. $\mathrm{m}_{\mathrm{c}}$ and a generate a "bare" $\bar{c} \bar{c}$ spectrum, which is renormalized by the coupling to the continuum. For fixed $\mathrm{m}_{\mathrm{u}} \simeq 1 / 3 \mathrm{GeV}$ and $\mathrm{m}_{\mathrm{s}} \simeq 1 / 2 \mathrm{GeV}, \mathrm{m}_{\mathrm{c}}$ and a are adjusted until the Green's function

$$
\mathscr{G}_{\mathrm{mn}}=(\mathrm{W}-\varepsilon-\Omega(W))_{\operatorname{mn}}^{-1}
$$

has $\mathrm{J}^{\mathrm{PC}}=1^{--}$poles at 3.095 and $3.684 \mathrm{GeV}$ (corresponding to $\psi^{-}$and $\psi^{\prime}$ ), and a residue at $3.095 \mathrm{GeV}$ such that the $\psi$ electronic width is $5.3 \mathrm{keV}$. The computed $\psi^{-}$electronic width is $3.4 \mathrm{keV}$, which is the same as found in the basic model (Table 3) and $\sim 1.7$ times the observed value. Table 7 contains a 1 ist of the "bare" and renormalized masses of the spin-triplet states below charm thresh01d. Especially noteworthy are the large downward shifts in masses and the very small splitting of the ${ }^{3} \mathrm{P}_{J}$ states. The first effect shows that $\mathrm{H}_{\mathrm{I}}$ is by no means a weak perturbation. The second is a consequence of the fact that the only spin-dependence comes from the split thresholds and will be sizable only for states very near (within $\approx 50-100 \mathrm{MeV}$ ) these thresholds. 
Table 7

Mass shifts in the coupled-channel mode1 (68). Parameters used are $m_{c}=1.69 \mathrm{GeV}, a=1.80 \mathrm{GeV}^{-1}, \mathrm{~m}_{\mathrm{u}}=\mathrm{m}_{\mathrm{d}}=0.33 \mathrm{GeV}$ and $\mathrm{m}_{\mathrm{s}}=0.50 \mathrm{GeV}, D$ and $\mathrm{D}^{*}$ masses are taken from experiment while $\mathrm{M}_{\mathrm{F}}=2.00 \mathrm{GeV}$ and $\mathrm{M}_{\mathrm{F}^{*}}=2.14 \mathrm{GeV}$ are assumed.

\begin{tabular}{|c|c|c|c|}
\hline State & $\begin{array}{c}\text { Bare Mass } \\
\text { (MeV) }\end{array}$ & Mass Shift & $\begin{array}{l}\text { Renormalized } \\
\text { Mass }\end{array}$ \\
\hline$\psi$ & 3191 & -96 & 3095 \\
\hline$\psi^{\prime}$ & 3893 & -209 & 3684 \\
\hline$\psi^{\prime \prime}$ & 3976 & -208 & 3768 \\
\hline$x_{2}$ & 3622 & -170 & 3451 \\
\hline$x_{1}$ & 3622 & -180 & 3442 \\
\hline$x_{0}$ & 3622 & -191 & 3431 \\
\hline
\end{tabular}


2. RADIATIVE TRANSITIONS With the parameters determined by renomalization, the radiative transition rate for $\psi^{-} \rightarrow x_{J}+\gamma$, say, is computed as follows: Falling back on the (over-simplified) notation of Eq. (3.46), let $|\mathrm{N}\rangle=\left|\psi^{\prime}\right\rangle$, $|M\rangle=\mid x_{J}>$, and $j_{\lambda}=\frac{2}{3}\left(\bar{c} \gamma_{\lambda} c+\vec{u} \gamma_{\lambda} u\right)-\frac{1}{3}\left(\bar{d} \gamma_{\lambda} d+\bar{s} \gamma_{\lambda} s\right)$ the electromagnetic current. The E1 transition amplitude is (68)

$$
\begin{aligned}
& \left\langle M\left|j_{\lambda}\right| N\right\rangle=\sum_{m, n} a_{m M}^{*} a{ }_{n N}\left\langle m\left|j_{\lambda}\right| n\right\rangle+\int d \mu d \nu a_{\mu M}^{*} a_{\nu N}\left\langle\mu\left|j_{\lambda}\right| \nu\right\rangle \\
& +\sum_{n} \int d v\left[a_{v M}^{*} a_{n N}\left\langle v\left|j_{\lambda}\right| n\right\rangle+a^{*}{ }_{n M} a_{v N}\left\langle n\left|j_{\lambda}\right| v\right\rangle\right] \text {. }
\end{aligned}
$$

The first term on the right in Eq. (3.55) includes only the parts of $\psi^{\prime}$ and $x_{J}$ in the discrete $(\bar{c})$ sector, with $\left\langle m\left|j_{\lambda}\right| n\right\rangle$ computed just as in the potential model without coupling to the continuum. The second term, involving the continuum components of $\psi^{-}$and $x_{J}$, contains electromagnetic transition matrix elements of charmed mesons; these are taken from standard quark-model calculations. The third (cross) term involves a transition between the discrete and continum sectors under the action of $j_{\lambda}$.

In Iieu of some long and not-very-illuminating formulae for the terms in Eq. (3.55), a few remarks on their relative importance are offered. The most important contribution to the discrete-sector terms is obviously the diagonal one: $|\mathrm{n}\rangle=\left|2^{3} \mathrm{~S}_{1}\right\rangle$ and $|\mathrm{m}\rangle=\left|1^{3} \mathrm{P}_{\mathrm{J}}\right\rangle$. The next single most important contribution to this set comes from $|n\rangle=\left|1^{3} D_{1}\right\rangle$, i,e, the mixing of ${ }^{3} S_{1}$ and ${ }^{3} D_{1}$ states due to nearby spin-split thresholds, and this is rather sensitive to the precise position of the DD and $\overrightarrow{D D}^{*}$ thresholds. The $S-D$ mixing is most important for $\psi^{-} \rightarrow x_{0}+\gamma$ because of a large Clebsch-Gordan coefficient for $1^{3} D_{1} \rightarrow 1^{3} P_{0}+\gamma[$ see Eq. (3.26)]. Because of energy denominators, the continuun-sector terms are dominated by the nearest threshold accessible to both $\psi^{-}$and $x_{J^{*}}$. Thus, the continuum is considerably more important (roughly a factor 
of two in amplitude) for $x_{0}$ than for $x_{1}$ and $x_{2}$ because $x_{0} \rightarrow \bar{D}$ in an S-wave, while $x_{1} \rightarrow \bar{D} \bar{D}$ is forbidden and $x_{2} \rightarrow \bar{D} \bar{D}$ is suppressed by a $D$-wave factor. In amplitude, the continum contribution to $x_{0}$ is $v 1 / 2$ the diagonal contribution and of the same sign. Finally, the mixed terms are practically negligible.

For the MI transitions, only the discrete-sector terms have been computed so far. They are obtained from the standard formula $(72,68)$

$$
\Gamma\left(\psi_{\mathrm{N}} \rightarrow \eta_{\mathrm{C}, \mathrm{M}}+\gamma\right)=\frac{16}{27} \underset{\mathrm{c}}{\alpha \frac{\omega^{3}}{\mathrm{~m}}}\left|\sum_{\mathrm{n}} a_{\mathrm{nN}} a_{\mathrm{nM}}^{*}\right|^{2}
$$

i.e. only nonhindered terms (same principal quantum number) are kept. The overlap factor $\sum_{n} a_{n N} a_{n i d}^{*}$ is 0.7 for $\psi^{\prime}+\pi_{c}^{\prime},-0.13$ for $\psi^{\prime} \rightarrow n_{c},-0.05$ for $\eta_{c}^{\prime} \rightarrow \psi$, and 0.9 for $\psi \rightarrow \eta_{c}$, where $\eta_{c}$ and $\eta_{c}^{-}$were taken to lie at 2.8 and $3.45 \mathrm{GeV}$ for the purpose of this calculation. For the "hindered" Ml transition amplitudes, one may reasonably expect the neglected terms to be comparable to those so far compated.

The final results for El and M1 transition rates are displayed in Table 8 . Compared with the results of the potential model (Tables 4,5 ), the El rates show a modest improvement, though they are still one to two standard deviations from experiment. Once again the $M 1$ rates bear no resemblance to those observed for $\psi^{\prime} \rightarrow \gamma \chi(3455), x(3455) \rightarrow \gamma \psi$, and $\psi \rightarrow \gamma x(2830)$. Taking this together with the unexpectedly large hyperfine splittings, there can no longer be any doubt that something very important is missing from the charmonium model or that the identification of these states as hyperfine partners of $\psi^{\prime}$ and $\psi$ is wrong. 3. CHARMED MESON PRODUCTION IN $\mathrm{e}^{+} \mathrm{e}^{-}$ANNIHILATION The essence of the modei for $\Delta R$ is that charmed meson production is a quasi-two-body process mediated by those $\bar{c} \bar{c}$ states which couple to the photon. It is thus in the spirit of vectormeson dominance (97), generalized to include coupled-channel mixing. The quasitwo-body hypothesis, which has proved to be correct for center-of-mass energy 
Table 8

Radiative transition rates in the coupled-channel model (68). Parameters $\rightarrow$ used are given in Table 7 .

$\begin{array}{lc}\text { Mode } & \text { Width }(\mathrm{keV}) \\ \psi^{\prime} \rightarrow \gamma x_{2} & 19 \\ \psi^{\prime} \rightarrow \gamma x_{1} & 28 \\ \psi^{\prime} \rightarrow \gamma x_{0} & 37 \\ \psi^{\prime} \rightarrow \gamma \eta_{c} & 21 \\ \psi^{\prime} \rightarrow \gamma \eta_{c} & 12 \\ \psi^{\prime} \rightarrow \gamma n_{c}^{\prime} & 8 \\ \eta_{c}^{\prime} \rightarrow \gamma \psi & 0.1\end{array}$


W $\leqslant 4.4 \mathrm{GeV}$, implies that charmed meson spectroscopy can be readily and accurately carried out by studying the invariant mass recoiling against D (or F) in the reaction $\mathrm{e}^{+} \mathrm{e}^{-} \rightarrow \mathrm{D}(\mathrm{F})+$ anything.

For a charmed quark charge of $2 / 3, \Delta \mathrm{R}$ is given by (68)

$$
\Delta \mathrm{R}(\mathrm{W})=\frac{32 \pi}{\mathrm{W}^{2}} \sum_{\mathrm{m}, \mathrm{n}} \psi_{\mathrm{m}}(0)\left[\frac{\mathscr{G}^{+}\left(\Omega^{\dagger}-\Omega\right)}{2 i}\right]_{\mathrm{mn}} \Psi_{\mathrm{n}}(0)
$$

where the quantity in brackets is the absorptive part of the Green's function in Eq. (3.54). The $\Psi_{\mathrm{n}}(0)$ are the wave functions at zero ce-separation of the discrete sector states. Since $\Omega$ is really a sum over the allowed continum channel types, $\nu=\mathrm{D}^{\circ} \overline{\mathrm{D}}^{\mathrm{o}}, \mathrm{D}^{+} \mathrm{D}^{-}$, etc., $\Delta \mathrm{R}$ may be written as a sum over exclusivechannel ratios, $R_{\nu}$. Since $\Psi(0) \neq 0$ only for $S$-wave states in this model,

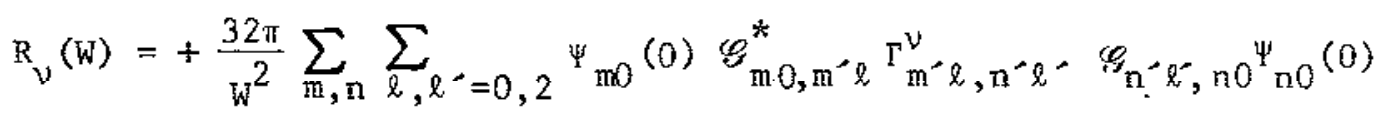

where the orbital quantum number $\ell, \ell^{-}=0,2$ has been made explicit. $\mathrm{F}_{\mathrm{m}}^{\nu} \ell, \mathrm{n}^{\prime} \ell^{\prime}$ is defined in Eq. (3.49), and it is the only factor in Eq. (3.58) which varies from one channel to the next-through its dependence on the momentum $p_{v}$, the intrinsic angular momenta, and the constituent quark masses of the outgoing charmed mesons (see Eq. (3.53)).

The reason for including ${ }^{3} \mathrm{D}_{1}$ levels, $\ell$ or $\ell^{*}=2$, in the orbital sum in $R_{v}$ is this: As mentioned, the only dependence on total quark spin in the calculation of $\Omega$ and $\mathscr{B}$ enters through the use of spin-split thresholds. This induces a mixing between nearby $S-$ and D-states which can show up as a D-state resonance pole in off-diagonal elements such as $\mathbb{B}_{20,12}$ This mixing is strongest when a ${ }^{3} D_{1}$ pole sits in the middle of a set of spin-split thresholds (e.g. at $W \simeq 3.8 \mathrm{GeV}$ ), and is considerably weaker when it is far from such 
thresholds (so that, e.g., D and $\mathrm{D}^{*}$ look degenerate).

Fig. 7 shows a graph of $\Delta R$ taken from Ref. 94. Completed some time before charmed meson masses were accurately measured, it assumed the thresholds $\mathrm{W}_{\mathrm{DD}}=3.730, \mathrm{~W}_{\mathrm{DD}} *=3.885, \mathrm{~W}_{\mathrm{D}}{ }_{\mathrm{D}}^{*}=4.040, \mathrm{~W}_{\mathrm{FF}}=4.00, \mathrm{~W}_{\mathrm{FF}} *=4.15$, and $\mathrm{W}_{\mathrm{F}} \mathrm{F}^{*}=$ 4.30 GeV. For comparison the most recent data from the various collaborations at SPEAR $(31,32)$ and at DORIS $(33,34)$ is shown fn Figs. 8-I1.

The prediction of the parameters of $\psi "(3772)$ more than a year before its discovery must be regarded as the greatest success of the coupled-channe1 model, especially in view of the fact that all attempts so far to understand the spin-dependent forces in charmonium have failed to give the requisite $2^{3} \mathrm{~S}_{1}-1^{3} \mathrm{D}_{1}$ mixing by more than an order of magnitude. Typical predictions of $\Gamma_{e}$ for this state based on the tensor force in a Brelt Hamiltonian are $\vartheta 20 \mathrm{eV}$. The predicted mass and hadronic width of $\psi^{\prime \prime}$ agree, within errors, with the measured values (see Table 1). The predicted electronic width of about $150 \mathrm{eV}$ is 2.5 times smaller than that reported by the SLAC-LBL collaboration $(370 \pm 90 \mathrm{eV})(90)$ while nearly the same as that measured by the DELCO group (180 $\pm 45 \mathrm{eV})$ (91). As we shall see in Sec. IV., the most important feature of $\psi^{: 2}$ is that it decays exclusively to $\mathrm{DD}$, providing a unique, highprecision setting in which to study these mesons.

Comparison of the theoretical curve with $\mathrm{R}$-data for energies $\mathrm{W}$ between 3.8 and $4.2 \mathrm{GeV}$ shows only qualitative agreement between the two. Points of agreenent include: (1) The dip in $\Delta \mathrm{R}$ to zero near $3.8 \mathrm{GeV}$ due, in the model, to the vector-meson dominated production. (2) The rise in $\Delta \mathrm{R}$ near $3.95 \mathrm{GeV}$. This is the $\mathrm{DD}^{*}$ threshold in the model calculation, but there $\Delta \mathrm{R}(\simeq 1)$ is only about one-half the measured value ${ }^{12}$. (3) The sharp rise in Fig 7 is due to the concurrence of the important $\mathrm{D}^{*} \overline{\mathrm{D}}^{*}$ threshold with the $3^{3} \mathrm{~S}_{1}$ charmonium level. The corresponding $r$ ise in the data, culminating in $\psi(4028)$, is considerably 
sharper $(\triangle \mathrm{R} \simeq 3$ in about $30 \mathrm{MeV})$. (4) The dip in exclusive $\mathrm{D} \overline{\mathrm{D}}$ production at this resonance is due to a zero in the $3^{3} \mathrm{~S}_{1} \rightarrow \mathrm{D} \overline{\mathrm{D}}$ form factor near $\mathrm{p}_{\mathrm{D}} \approx 750 \mathrm{MeV}$. This sfriking prediction of the model $(94,68,98)$ is confirmed experimentally. Study of the mass distribution recolling against observed D's at $4.028 \mathrm{GeV}$ gives the relative exclusive-channel ratios as (99).

$$
\begin{aligned}
& R\left(D^{*_{\mathrm{O}}}{ }^{*_{\mathrm{O}}}\right): R\left(\mathrm{D}^{{ }_{\mathrm{O}}} \overline{\mathrm{D}}^{\mathrm{O}}+\mathrm{D}^{\mathrm{O}} \overline{\mathrm{D}}^{*_{\mathrm{O}}}\right): \mathrm{R}\left(\mathrm{D}^{\mathrm{O}} \overline{\mathrm{D}}^{\mathrm{O}}\right) \\
& =1.00 \pm 0.10: 0.85 \pm 0.09: 0.10 \pm 0.06
\end{aligned}
$$

This preference of $\psi(4028)$ for $D^{*-*}$, despite the Iimited phase space, has been interpreted by some authors $(77,100)$ as an indication that $\psi(4028)$ is an almost bound state of these two mesons-a $D^{*} \bar{D}^{*}$ molecule. It is difficult to test this rather ad hoc hypothesis because no model of such objects exists which can be relied upon for further predictions. In the meantime, the existence of a near zero in $\overline{D D}$ production near $4.028 \mathrm{GeV}$ can be tested by careful study of this region, and will further establish the notion of quarks through the observation of a node in their bound-state wave functions.

Above $W \simeq 4.1 \mathrm{GeV}$, the mode1 calculation breaks down bad1y, and bears little resemblance to the data. In particular, the enhancement neax $4.15 \mathrm{GeV}$, the dip at $4.3 \mathrm{GeV}$, and the obvious resonance $\psi(4414)$ are all beyond the reach of the model as presently constituted. If $\psi(4028)$ is indeed the (highly distorted) $3^{3} \mathrm{~S}_{1}$ charmonium level, then the spectroscopy of the naive potential model would lead one to interpret the enhancement at $4150 \mathrm{MeV}$ as the $2^{3} \mathrm{D}_{1}$ state and the resonance at $4414 \mathrm{MeV}$ as the $4^{3} \mathrm{~S}_{1}$ state. But this is perhaps pushing the naive model too far. Most of its assumptions are questionable for such high excitations, and even the nonrelativistic spectroscopic notation may be meaningless.

Finally, it should be mentioned that several other models predict states in 
this region beyond those expected in the linear potential model. To mention two examples: (1) The model of Giles and Tye (78), in which the cc pair is bound by a string with dynamical degrees of freedom, expects a number of levels corresponding to vibrational excitations of the string. No prediction is made for the leptonic width of these new states, so that their observability is an open question. (2) The (essentially) logarithmic potential proposed by a number of authors $(101,102,86)$, has a greater level density than does the linear model, and predicts the $4^{3} \mathrm{~S}_{1}$ and $5^{3} \mathrm{~S}_{1}$ levels at 4.25 and $4.41 \mathrm{GeV}$, respectively. F. BEYOND CHARMONIUH

The recent discovery at Fermilab of enhancements in the $\mu$-pair invariant mass near $M_{\mu^{+}}{ }^{-} \simeq 10 \mathrm{GeV}$ (23) is widely interpreted as solid evidence for the existence of a new quark, $Q$, with mass $\mathrm{m}_{\mathrm{Q}} \approx 5 \mathrm{GeV}$. The data (Fig. 12) shows clearly two, and possibly three, resonances, called $T, T^{\prime}$, and $T^{\prime \prime}$. Assuming these to have zero width (consistent with the experimental-resolution of $\sim 200$ $\mathrm{MeV})$, a fit to the data gives their masses as

$$
\begin{aligned}
& M_{T}=9.40 \pm 0.013 \mathrm{GeV} \\
& M_{\mathrm{T}^{\prime}}=10.01 \pm 0.04 \mathrm{GeV} \\
& M_{\mathrm{T}^{\prime}}=10.40 \pm 0.12 \mathrm{GeV} .
\end{aligned}
$$

While the statistical significance of $T$ " is still not large, the obvious interpretation is that these are the ground and first two radialiy excited ${ }^{3} \mathrm{~S}_{1}$ states of a QQ̄ system. ${ }^{13}$

Since they are narrow enough to have appreciable $\mu^{+} \mu^{-}$branching ratios, they must all lie below the threshold for decay into a pair of mesons $\bar{Q}+\bar{Q} q$ containing one new and one old quárk $(q=u, d)$ each. Two other striking features of these states which are readily inferred from the data are these:

(i) The $2^{3} \mathrm{~s}-1^{3} \mathrm{~s}$ mass difference

$$
M_{\mathrm{T}^{-}}-\mathrm{M}_{\mathrm{T}}=610 \pm 50 \mathrm{MeV}
$$


is, within errors, the same as in charmonium, $\mathrm{M}_{\psi^{-}}-\mathrm{M}_{\psi}=589 \mathrm{MeV}$. As we shall see, this is about $150 \mathrm{MeV}$ larger than expected if one adheres to the "standard" potential $v_{0}$ in Eq. (3.8). (ii) The ratio of the observed $\mu^{+} \mu^{-}$signals at $\tau^{\prime}$ and $T$ is

$$
R_{\mu}=\left.\frac{B\left(\Upsilon^{-}+\mu^{+} \mu^{-}\right) d \sigma / d y}{B\left(T+\mu^{+} \mu^{-}\right) d \sigma / d y}\right|_{y=0}=0.37 \pm 0.04
$$

The corresponding value of $R_{\mu}$ for $\psi^{\prime}$ and $\psi$ production is about 0.02 (59). This strongly suggests (I03) that $B\left(\Upsilon^{-}+\mu^{+} \mu^{-}\right) \gg B\left(\psi^{-}+\mu^{+} \mu^{-}\right)$and, therefore, that $\Gamma\left(\Upsilon^{-} \rightarrow \Upsilon+\right.$ anything $) \ll \Gamma\left(\psi^{\prime} \rightarrow \psi+\right.$ anything $) \simeq 130 \mathrm{keV}$. These features of the T-system will receive considerable attention in the following discussion.

It hardly need be emphasized that bound systems of quarks heavier than charm will provide critical tests of the foundations of the charmonium model-gluon counting and the use of a nonrelativistic potential. Furthermore, the observed spectrum and branching ratios for radiative and direct decays will sharpen our knowledge of the form of this potential, since it is expected to be largely independent of $\mathrm{m}_{\mathrm{Q}}$. And, of course, the relative strength of radiative and leptonic decays to purely hadronic ones will help determine the new quark's charge.

These issues and more have already sparked considerable theoretical interest in the $\Upsilon$-system where, as we just mentioned, the prediction (104) of the standard potential for $\mathrm{M}_{\mathrm{T}}$ - - $\mathrm{M}_{\uparrow}$ appears to have failed. But a complete test of the form of the potential requires a comparison with experiment of its expectations for the myriad of branching ratios and absolute widths, as well as the details of the spectrum accessible only to $\mathrm{e}^{+} \mathrm{e}^{-}$storage ring experiments. And preliminary to making meaningful predictions, one must decide the relative positions of the ground state $Q \bar{Q}$ and the threshold for OZI-allowed decays. 
Only then can one know in a given model how many states are bound (i.e. narrow) and what transitions among these should be observed.

Eichten and Gottfried ( 104 ) have addressed the question of the $m_{Q}$-dependence of the threshold and ground-state energies. While their arguments are, strictly speaking, valid only in the $\mathrm{m}_{\mathrm{Q}} \rightarrow \infty$ limit, errors should be small so long as

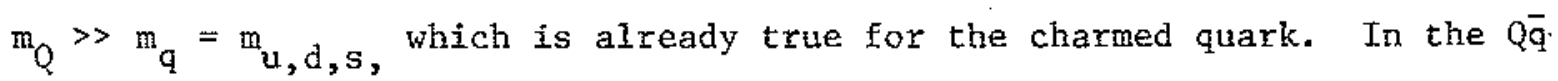
system, for example, the reduced mass $\mu=m_{Q} m_{q} /\left(m_{Q}+m_{q}\right) \approx m_{q}$ and the dynamics is essentially independent of $\mathrm{m}_{\mathrm{Q}}$. The mass of the Q $\bar{q}$ ground-state pseudoscalar is then the sum of $\mathrm{m}_{\mathrm{Q}}+\mathrm{m}_{\mathrm{q}}$, the binding energy (a function of $\mathrm{m}_{\mathrm{q}}$ only), and a correction due to the ${ }^{3} S-{ }^{1} S$ hyperfine splitting. This last term decreases like $\mathrm{m}_{\mathrm{Q}}{ }^{-1}$ in a heavymlight system, so that the threshold energy $\mathrm{W}_{\mathrm{Q}}$ may be written as

$$
W_{Q}=2\left[M_{D}+m_{Q}-m_{C}+\frac{3}{4}\left(1-\frac{m_{C}}{m_{Q}}\right)\left(M_{D *}-M_{D}\right)\right]
$$

In the $Q \bar{Q}$ system, the mass $M_{1}$ of the ${ }^{3} S$ ground state is

$$
M_{1}=E_{1}(Q \bar{Q})+E_{0}\left(m_{Q}\right)
$$

where $E_{1}$ is the ground state eigenvalue and $E_{0}$ is the zero of energy, whose definition is not completely obvious in an infinitely rising potential such as $\mathrm{V}_{0}$. Writing

$$
E_{O}=2 m_{Q}+\Delta\left(m_{Q}\right)
$$

all that is known about $\Delta$ is (i) $\Delta\left(m_{c}\right) \cong-205 \mathrm{MeV}$ and (ii) $m_{Q}{ }^{-1}\left(_{Q}\right) \rightarrow 0$ as $\mathrm{m}_{\mathrm{Q}}+\infty$. Eichten and Gottfried interpolate ${ }^{14}$ using $\Delta\left(\mathrm{m}_{\mathrm{Q}}\right)=\Delta\left(\mathrm{m}_{\mathrm{c}}\right) \mathrm{m}_{\mathrm{c}} / \mathrm{m}_{\mathrm{Q}}$. Using the potential $\mathrm{V}_{\mathrm{o}}$ in $\mathrm{Eq}$. (3.8), with essentially the same parameters as in Eq. (3.9), Eichten and Gottfried computed the excitation spectrum shown in Fig. 13. Using Eqs. (3.63)-(3.65), the quark mass appropriate 
to the $T$ system is $\mathrm{m}_{\mathrm{Q}}=4.6 \mathrm{GeV}$, and the threshold for OZI-allowed decays is $900 \mathrm{MeV}$ above the $\mathrm{T}$. Thus, they predicted three bound ${ }^{3} \mathrm{~S}$ states, as seems to be the case, with masses

$$
\begin{aligned}
& M_{T}-M_{T} \simeq 450 \mathrm{MeV}, \quad M_{T} \prime-M_{T} \simeq 750 \mathrm{MeV} \\
& M\left(Q \bar{u} ; 1^{I} S_{0}\right) \simeq 5.16 \mathrm{GeV}, \quad\left(\text { for } m_{c}=1.37 \mathrm{GeV}\right) \\
& M\left(Q \bar{u} ; 1^{3} S_{1}\right)-M\left(Q \bar{u} ; 1^{1} S_{0}\right) \simeq 100 \mathrm{MeV} .
\end{aligned}
$$

One possible explanation for the apparent failure of the highIy motivated Iinear + Coulomb model to predict correctly the $T^{\prime}-T$ and $T^{\prime \prime}-T$ separations is this: While good arguments exist for both the small and large $r$ behavior of the potential, it may well be that the systems under investigation, $\psi$ and $\Upsilon$, see mainly an intermediate range portion of the $\bar{Q}$ potential. This may be neither linear nor Coulomb and, in any case, no arguments exist which give a clue to its shape.

Whatever the reason for failure, the large $T^{\prime}-T$ mass difference has caused renewed interest in alternative forms of the potential. One choice, the logarithmic potential, studied some time ago by Machacek and Tomozawa (101) and more recently by Quigg and Rosner (102), is currently in vogue because of its peculiax property that the Q $\bar{Q}$ excitation spectrum is independent of the Q-rass. Thus, by fitting to the $\psi$-system, Quigg and Rosner find

$$
V_{1}(r)=0.733 \mathrm{GeV} \text { in }\left(r / r_{0}\right)
$$

with $r_{o}$ an arbitrary constant, and $m_{c}=1.1 \mathrm{GeV}$. The spectrum of the first few excited $Q \bar{Q}$ levels may be found from Fig. 13 by drawing horizontal lines through the dots corresponding to members of the charmonium family. The predictions of the two models for charmonium start to deviate around the $3^{3} \mathrm{~S}_{1}$ state; 
in the logarithmic potential,

$$
M\left(\mathrm{cc} ; 3^{3} \mathrm{~s}_{1}\right)=4.03 \mathrm{GeV}, \quad M\left(\overline{c c} ; 4^{3} \mathrm{~s}_{1}\right)=4.25 \mathrm{GeV}
$$

compared to 4.17 and $4.6 \mathrm{GeV}$, respectively, in the $\mathrm{V}_{0}$ model. The separation between the ground state and ozI threshold will not be the same as in the Efchten-Gottfried calculation because quark masses appropriate to $V_{1}$ will differ somewhat from those for $\mathrm{V}_{0^{\circ}}$ In particular, Quigg and Rosner find $\mathrm{m}_{\mathrm{Q}} \cong 4.5 \mathrm{GeV}$ so that, using Eq. (3.63), they predict

$$
\mathrm{M}\left(\overline{\mathrm{u}} ; \mathrm{I}^{\underline{l}} \mathrm{~S}_{0}\right)=5.33 \mathrm{GeV} \quad \text { (for } \mathrm{m}_{\mathrm{c}}=1.1 \mathrm{GeV} \text { ) }
$$

and that three to four ${ }^{3} \mathrm{~S}$ states will be bound.

Celmaster, Georgi, and Machacek ( 86 ) have proposed still another potential inspired by the linear + Coulomb model:

$$
\begin{aligned}
& v_{2}=\frac{-8 \pi}{27 r}\left[\ln \left(\frac{1}{r \Lambda \mathrm{e} \gamma}\right)\right]^{-1}+\left(1-\mathrm{e}^{-\mathrm{Ar}}\right) \mathrm{r} / 2 \pi+\mathrm{E}_{0} \\
& \Lambda=0.50 \mathrm{GeV}, \quad \gamma=0.577, \quad \mathrm{~A}=0.16 \mathrm{GeV}, \quad \mathrm{E}_{0}=0.39 \mathrm{GeV}
\end{aligned}
$$

The logarithm and coefficient in the short-range part of $v_{2}$ is motivated by appeal to asymptotic freedom (however, the argument of the logarithm has been modified). The Iinear potential strength, $(2 \pi)^{-1} \mathrm{GeV}^{2}$, is taken from the slope of the Regge trajectory. The new, internediate-range part, $(r / 2 \pi) \exp (-\mathrm{Ar})$, is chosen arbitrarily. The parameters in Eq. (3.68) are detemined by fitting to the spectra of light as well as heavy (cic) mesons-a very questionable procedure for any nonrelativistic potential. The charmed quark mass that results is $m_{c}=1.98 \mathrm{GeV}$. With al1 parameters determined (including the zero of energy,

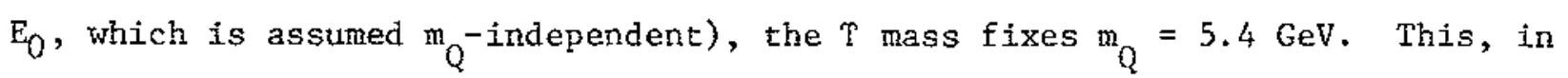
turn, leads to

$$
M\left(Q_{u} ; 1^{1} \mathrm{~S}_{0}\right)=5.35 \mathrm{GeV} \quad \text { (for } \mathrm{m}_{\mathrm{c}}=1.98 \mathrm{GeV} \text { ) }
$$


and the prediction that $3-4^{3} \mathrm{~s}$ levels will be bound below OZI threshold. The potentials $V_{0}, V_{1}, V_{2}$ are plotted in Fig. 14, and the radial probability $P(r) d r$ for the $\psi\left(1^{3} S_{1}\right)$ and $T\left(1^{3} S_{1}\right)$ states as determined by $V_{0}$ is shown in Fig. 15. Several remarks immediately follow from these figures:

(i) $V_{1}$ and $V_{2}$ are practically congruent, and so the level spacing determined by $\mathrm{V}_{2}$ is almost independent of $\mathrm{m}_{\mathrm{Q}}$ and fits well what is known about the $T$ spectrum. There will be small differences in the wave functions, hence the rates, predicted by the two models because of the different values of $\mathrm{m}_{Q}$.

(ii) AII three potentials are nearly congruent in the region in which the $\psi$ wave function is large, $0.2 \mathrm{fm} \leqslant x \leqslant 0.6 \mathrm{fm}$. Thus, it is not surprising that all three give the same spectrum of low-lying c' levels and roughly comparable strengths for radiative decays. (iii) Over most of the region in which the $T$ wave function is concentrated, 0.1 fm $\leqslant r \leqslant 0.3 \mathrm{fm}$, there is considerable difference between the shape of $V_{0}$ and of $V_{1,2}$. It is not surprising, therefore, that the $v_{0}$ spectrum is quantitatively different from the $v_{1}, v_{2}$ spectra here.

The predictions of Refs. 104, 102, 86 for $\Upsilon$ and $\Upsilon$ branching ratios and total widths are listed in Table 9. To estimate $\mathrm{T}^{\prime} \rightarrow \mathrm{T} \pi \pi+\mathrm{T} \eta$, we have used Gottfried's scaling relation (71)

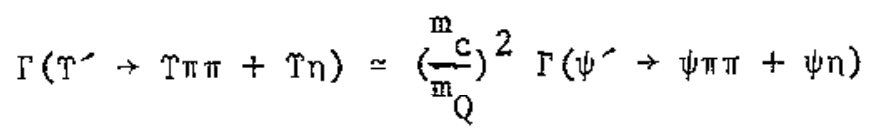

with $\left(\mathrm{m}_{\mathrm{c}} / \mathrm{m}_{\mathrm{Q}}\right)^{2} \simeq 1 / 10$. Since cascade radiative decays will constitute only a very small part of $\mathrm{T}^{-}+\mathrm{T}$ transitions, the suppression in Eq. (3.72) goes a long way toward explaining the unexpectedly large value of $\mathrm{R}_{\mu}$ in Eq. (3.62) While very little is known of the details of the $\Upsilon$-system at present, we can 
Table 9

Predicted $T$ and $\Upsilon$ transition rates (104, 102, 86)

\begin{tabular}{|c|c|c|c|c|c|c|c|}
\hline \multirow[b]{2}{*}{ Particle } & \multirow[b]{2}{*}{ Mode } & \multicolumn{2}{|l|}{$v_{o}$} & \multicolumn{2}{|c|}{$v_{1}$} & \multicolumn{2}{|c|}{$\mathrm{v}_{2}$} \\
\hline & & $\left|\mathrm{e}_{\mathrm{Q}}\right|=1 / 3$ & $2 / 3$ & $1 / 3$ & $2 / 3$ & $1 / 3$ & $\underline{2 / 3}$ \\
\hline $\mathrm{p}^{\prime}$ & $\ell^{+} \ell^{-}(\ell=e, \mu, \tau)$ & 0.45 & 1.8 & 0.5 & 1.9 & 1 & 4 \\
\hline & $\sum_{J}\left(\gamma+1^{3} P_{J}\right)$ & 6.8 & 27 & 10 & 40 & 8.8 & 35 \\
\hline & $T \pi \pi+T \eta$ & $\sim 12$ & $\sim 12$ & u2 & v12 & $\sim 12$ & 012 \\
\hline & Hadrons (direct) & 8.6 & 8.6 & 9.1 & 9.1 & 19 & 19 \\
\hline & Hadrons (2nd order E.M.) & 1.9 & 7.6 & 2.0 & 8.0 & 4.2 & 17 \\
\hline & A11 & 431 & $v 60$ & $\approx 35$ & $\sim 75$ & ?47 & 295 \\
\hline $\mathrm{T}$ & $\ell^{+} \ell^{-}(l=e, \mu, \tau)$ & 0.7 & 2.7 & 1.1 & 4.3 & 2.5 & 10 \\
\hline & Hadrons (direct) & 14.5 & 14.5 & 23 & 23 & 52 & 52 \\
\hline & Hadrons (2nd order E.M.) & 2.8 & 11.4 & 4.5 & 18 & 10 & 40 \\
\hline & A11 & 219 & $\checkmark 34$ & $\sim 31$ & 0.54 & $\% 70$ & vI22 \\
\hline
\end{tabular}


look forward in the next few years to a flood of data from the new $e^{+} e^{-}$storage rings at Corne11, DESY, and SLAC. It can only be hoped that out of all this will come a clearer picture of the "correct" phenomenological potential for heavy quark binding and, indeed, of the foundations of the charmonium model. Beyond this, we need a better understanding of the corrections to the naive model due to quark spin and the inevitable presence of light-quark and gluonic degrees of freedom. The experimental study of $Q \bar{Q}$ systems is essential, but equally so is progress in understanding the structure of quantum chromodynamics itself--a theory which is still very much in its infancy. 


\section{CHARMED HADRONS}

Direct evidence for the existence of charmed particles was announced in the summer of 1976, a year and a half after discovery of the $\psi / J(22)$. For an early discussion of charm phenomenology which anticipated many of the recent discoveries, the reader is referred to the paper of Gaillard, Lee and Rosner (106). The properties of the charmed hadrons observed so far are shown in Table 10. Several other recent theoretical and experimental reviews are available $(59,107,108)$.

\section{A. THEORY}

1. SPECTROSCOPY Since all the charmed particles contain one or more light quarks, relativistic motion will very likely make them much more difficult to treat with a potential model than charmonium. A detailed discussion of attempts at light quark dynamics falls outside the scope of our paper. The point we want to make here is that nothing terribly surprising seems to be going on. The experiments have largely confirmed the qualitative expectations of the charm mode1.

The masses of Table 10 can, in fact, be qualitatively understood in the most naive form of the nonrelativistic quark model by adding appropriate amounts of

$$
\mathrm{m}_{\mathrm{c}}=\frac{1}{2} \mathrm{M}_{\psi} \simeq 1.55 \mathrm{GeV} \quad \mathrm{m}_{\mathrm{u}}=\mathrm{m}_{\mathrm{d}} \simeq 0.33 \mathrm{GeV}
$$

$$
\mathrm{ta}_{\mathrm{S}} \simeq 0.46 \mathrm{GeV}
$$

This reproduces the psendoscaiar and $J^{P}=\frac{1}{2}^{+}$baryon masses reasonably well and the vector mesons require roughly an extra $150 \mathrm{MeV}$ hyperfine energy. Given the observed masses $M_{D} \simeq 1.865 \mathrm{GeV}$ and $M_{D^{*}} \simeq 2.005 \mathrm{GeV}$, one can do 
Table 10

Charmed Particle Properties. All data is taken from Ref. I08 which cites original work. Quark content is shown below particle name. D*t branching ratios are estimated (108) as described in the test for $\mathrm{m}_{\mathrm{l}} / \mathrm{m}_{\mathrm{c}}=\mathrm{m}_{\mathrm{p}} / \mathrm{m}_{\psi}$ (parenthetical numbers are for $\mathrm{m}_{\mathrm{q}} / \mathrm{m}_{\mathrm{C}}=0$ ).

$$
\begin{aligned}
& \frac{\text { Particie }}{D^{\circ}} \frac{I\left(\mathrm{~J}^{\mathrm{P}}\right)}{\frac{1}{2}\left(\mathrm{O}^{-}\right)} \frac{\text { Mass }(\mathrm{MeV})}{1863.3 \pm 0.9} \frac{\text { Decay Mode }}{\mathrm{K}^{-} \pi^{+}} \\
& \text {(cū) } \\
& \mathrm{K}^{\circ} \pi^{+}- \\
& \text {Branching Fraction } \\
& \mathrm{K}^{-} \pi^{+} \mathrm{O} \\
& \text { (\%) } \\
& 2.2 \pm 0.6 \\
& 4.0 \pm 1.3 \\
& 12 \pm 6 \\
& \mathrm{~K}^{-}+\pi^{+} \pi^{+} \\
& \mathrm{e}^{+} v_{\mathrm{e}}+\text { hadrons } \\
& 3.2 \pm 1.1 \\
& 210 \\
& \stackrel{\mathrm{D}^{+}}{(\mathrm{cd})} \\
& \frac{1}{2}\left(0^{-}\right) \\
& 1868.3 \pm 0.9 \quad \overline{\mathrm{K}}^{\mathrm{o}}{ }^{+} \\
& \mathrm{K}^{-} \pi^{+}+ \\
& \mathrm{e}^{+} v_{\mathrm{e}}+\text { hadrons } \\
& 1.5 \pm 0.6 \\
& 3.9 \pm 1.0 \\
& \text { 210 } \\
& \delta=\mathrm{M}_{D^{+}}-\mathrm{M}_{\mathrm{D}^{\circ}}=5.0 \pm 0.8 \mathrm{MeV} \\
& 2006 \pm 1.5 \\
& 45 \pm 15 \\
& \frac{1}{2}\left(1^{-}\right) \\
& 2008.6 \pm 1.0
\end{aligned}
$$

$(\mathrm{c}+\bar{s})$

$$
0\left(0^{-}\right)
$$

$$
2039.5 \pm 1.0
$$

$n \pi+$

$\mathrm{K}^{+} \mathrm{K}_{\pi}^{-}+$

(cs)

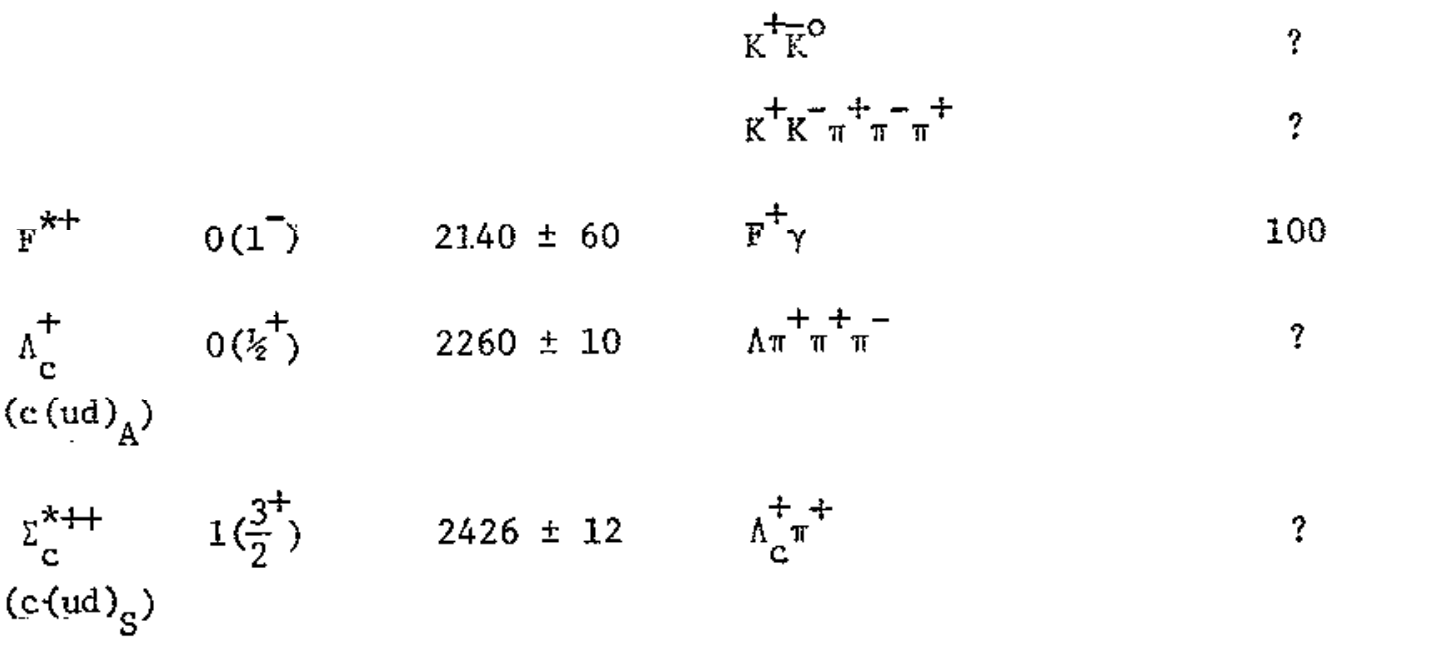


even better with

$$
\begin{aligned}
& M_{F^{*}}=M_{D^{*}}+M_{\phi}-M_{K^{*}}=2.13 \mathrm{GeV} \\
& M_{F}=M_{F}^{*}-\left(M_{D} *-M_{D}\right)=1.99 \mathrm{GeV}
\end{aligned}
$$

The reported $F$ and $F^{*}$ masses in Table 10 are reproduced nicely by this sum rule.

Mass splittings between $\mathrm{D}^{\circ}$ and $\mathrm{D}^{+}$and between $\mathrm{D}^{*_{0}}$ and $\mathrm{D}^{*+}$ have been measured (108) and are also interesting theoreticaliy. In fact, because the $D^{*}$ - D mass difference leads to an extremely sma11 Q-value for $D^{*} \rightarrow D^{\prime}$, the $\mathrm{D}^{\circ}-\mathrm{D}^{+}$and $\mathrm{D}^{* 0}-\mathrm{D}^{*+}$ splittings have important experimental consequences in sorting out the spectroscopy of the $D^{\prime} s$. In the nonrelativistic quark mode1, the splitting is the sum of the down-up mass difference and a contribution from single-photon exchange:

$$
\begin{aligned}
& M_{D^{+}}-M_{D^{\circ}}=m_{d}-m_{u}+\frac{2}{3} \alpha\left\langle\frac{l}{r_{D}}\right\rangle+\frac{2 \pi}{m_{C} m_{u}}\left|\Psi_{D}(0)\right|^{2}{ }^{-} \\
& M_{D^{*+}}-M_{D}^{* 0}=m_{d}-m_{u}+\frac{2}{3} \alpha\left[\left\langle\frac{1}{r}\right\rangle_{D}-\left.\left.\frac{2 \pi}{3 m_{c} m_{u}}\right|_{D}(0)\right|^{2}\right]
\end{aligned}
$$

These expressions can be evaluated using a current algebraic extraction of $\mathrm{m}_{\mathrm{d}}-\mathrm{m}_{\mathrm{u}}-(109)$ and an atomic quark model analogous to charmonium for the $\mathrm{D}$ mesons. The result is $M_{D^{+}}-M_{D^{\circ}} \simeq 7.0 \mathrm{MeV}$ and $M_{D^{*}}-M_{D^{*}} \simeq 6.5 \mathrm{MeV}$ to be compared with the experimental values of $5.0 \pm 0.8 \mathrm{MeV}$ and $2.6 \pm 1.8 \mathrm{MeV}$ respectively. An alternative estimate of these splittings using the MIT Bag model ( 92,110$)$ gives essentially the same result.

In addition to the S-wave charmed mesons, $P$ states should exist as well. Their masses have been estimated by Eichten et al (68) to be

$$
\begin{array}{ll}
\mathrm{M}_{\mathrm{D}}\left(1^{3} \mathrm{P}\right)=2.44 \mathrm{GeV} & \mathrm{M}_{\mathrm{D}}\left(1^{3} \mathrm{P}_{1}\right)=2.58 \mathrm{GeV} \\
\mathrm{M}_{\mathrm{D}}\left(1^{1}{ }_{\mathrm{P}}\right)=2.45 \mathrm{GeV} & \mathrm{M}_{\mathrm{D}}\left(1^{3} \mathrm{P}_{2}\right)=2.58 \mathrm{GeV}
\end{array}
$$


The evidence for these states (as well as the beautiful measurements of the $D$ and $F$ masses) will be discussed shortly.

2. DECAYS In the standard WS-GIMi model $(4,7)$, the hadronic current Eq. (1.7) leads to the selection rule $\Delta C=\Delta S= \pm I$ for charm decays. Ignoring $Q C D$ renormalization corrections, the complete effective Hamiltonian for charm decays in this model is

$$
\begin{aligned}
\mathscr{H}_{\Delta \mathrm{C}}= & \frac{\mathrm{G}_{\mathrm{F}}}{\sqrt{2}}\left[\cos \theta_{\mathrm{c}} \bar{c}_{\lambda}\left(1-\gamma_{5}\right) \mathrm{s}-\sin \theta_{\mathrm{c}} \bar{c} \gamma_{\lambda}\left(1-\gamma_{5}\right) \mathrm{d}\right] \\
& x \cdot\left[\cos \theta_{c} \overline{\mathrm{d}} \gamma^{\lambda}\left(1-\gamma_{5}\right) \mathrm{u}+\sin \theta_{c} \bar{s} \gamma^{\lambda}\left(1-\gamma_{5}\right) \mathrm{u}+\sum_{\ell=e, \mu} \bar{\ell} \gamma^{\lambda}\left(1-\gamma_{5}\right) \nu_{\ell}\right]+\text { h.c. }
\end{aligned}
$$

If one naively assumes that charmed hadron decays are processes in which only the constituent $c$ quark participates, one estimates from Eq. (4.5) the following relative rates (ignoring questions of phase space):

$$
\begin{array}{ll}
c \rightarrow \operatorname{sud}=3 \cos ^{4} \theta_{c} & . \\
c \rightarrow d u \bar{d}=3 \cos ^{2} \theta_{c} \sin ^{2} \theta_{c} & \\
c \rightarrow \operatorname{sus}=3 \cos ^{2} \theta_{c} \sin ^{2} \theta_{c} & \\
c \rightarrow d u \bar{s}=3 \sin ^{4} \theta_{c} & (\ell=e, \mu) \\
c \rightarrow \operatorname{sl}^{+} v_{\ell}=\cos ^{2} \theta_{c} & (l=e, \mu) \\
c \rightarrow d l^{+} v_{\ell}=\sin ^{2} \theta_{c} &
\end{array}
$$

The factor of 3 is due to a sum over the color of the quarks in the noncharmed piece of the hadxonic current. Ignoring all but $\Delta C=\Delta S$ transitions, one expects $D$ (and $F$ ) decays will be $60 \%$ nonleptonic and $40 \%$ semileptonic, divided equally between $e$ and $\mu$. All of these will involve a single kaon, which provides the outstanding signal for the presence of charm. Note, in particular, that $\Delta C=\Delta S$ implies that one should see a $D^{+}$signal in the exotic channel $\mathrm{K}^{-} \pi^{+} \pi^{+}$, but not in the nonexotic $\mathrm{K}^{+} \pi^{+} \pi^{-}$. Finally, the $\mathrm{D}$ lifetime is estimated 
in this mode1 to be

$$
\tau(D \rightarrow a 11)=\frac{1}{5}\left(\frac{m_{\mu}}{M_{D}}\right)^{5} \tau(\mu \rightarrow \text { ev } \bar{v})=10^{-13} \mathrm{sec}
$$

Thus visual observation of $D$ decays can be made only with high-resolution techniques using emulsions or streamer chambers (111).

More detailed studies of charmed meson decays have been made by Einhorn and Quigg ( 112 ) and by E11is et a1 (113), as well as by several other groups (114). These are based on the analyses of the operator structure of the nonleptonic weak Hamiltonian carried out by Gaillard and Lee and by Altarelli and Maiani (46). The nonleptonic Hamiltonian is found to consist of two pieces, one transforming as the $[20]$ representation under SU(4) (flavor), the other as $[84\}$. When decomposed with respect to SU(3) subgroups (the symmetry group of $\mathrm{u}, \mathrm{d}$, s quarks), one finds.

$$
\begin{aligned}
& {[\underline{\underline{2}}]=\underline{6} \oplus \underline{8} \oplus \underline{6}^{*}} \\
& \left.[\underline{84}]=\underline{6} \oplus\left\{\underline{3} \oplus \underline{15} \underline{I}_{M}\right\}+\underline{6}^{*} \oplus\left\{\underline{3}^{*} \oplus \underline{15} \underline{M}^{*}\right\}+\{\underline{1} \oplus \underline{8} \oplus \underline{\underline{2}}\}\right\}
\end{aligned}
$$

with square brackets used to distinguish representations of $S U(4)$ from those of $S U(3)$, and the subscript $M$ denoting a representation of mixed symmetry. The octet in the decomposition of $[20]$ is the $\Delta C=0,|\Delta S|=1$ operator responsible for nonleptonic $\mathrm{K}$-decay; its matrix elements are enhanced relative to the octet and $27-p l e t$ in $|84|$.

On this basis, Einhorn and Quigg argued that the $\Delta C= \pm 1$ pieces, $\underline{6}$ and $\underline{6}^{*}$ in $\{\underline{20}\}$ should have enhanced matrix elements relative to the $\Delta \mathrm{C}= \pm 1$ parts of $[84]$, name1y $3 \in 15 \mathrm{M}+\underline{3}^{*} \oplus 15_{M}^{*}$. (Actually, only $15 \mathrm{M} \oplus \underline{35_{M}^{*}}$ appear in the Hamittonian.) Now, part of this octet enhancement is due to the sign of anomalous dimensions appearing in the operator product expansion (41), while 
an appreciable further enhancement is due to incalculable matrix elements of the operator, i.e. it is of uncertain origin. Furthermore, some of the octet enhancement arises from the choice of renormalization point in the evaluation of the anomalous dimensions of the operators; this was taken to be $1 \mathrm{GeV}$ for K-decay ( 46 ), and assumed to be the same by Einhorn and Quigg. Ellis et al argue that this renormalization point should be taken higher when dealing with decays of charmed hadrons, say $\mathrm{m}_{\mathrm{C}} \sim 2 \mathrm{GeV}$. This, they claim, diminishes sextet enhancement of the $|\Delta C|=1$ Hamiltonian.

Now, all this has measurable consequences. Under the reasonable assumption that decays of high-mass states such as $D$ and $F$ are quasi-two-body, Einhorn and Quigg point out that sextet enhancement implies that $D^{+}$. has no Cabibbo enhanced decays $\left(x \cos ^{4} \theta_{c}\right.$ ) to a pair of pseudoscalars (such as $\overline{\mathrm{K}}^{0} \pi^{+}$) or a pair of vectors (Iike $\overline{\mathrm{K}}^{*_{0} \rho^{+}}$). The only Cabibbo-enhanced decays of $\mathrm{D}^{+}$then would be to a pseudoscalar plus a vector, say $\mathrm{D}^{+} \rightarrow \overline{\mathrm{K}}^{\circ} \rho^{+} \rightarrow \mathrm{K}^{\circ} \pi^{+}{ }^{\circ}$ and $\mathrm{D}^{+} \rightarrow \overline{\mathrm{K}}^{-\mathrm{O}_{\pi}}+\rightarrow \mathrm{K}^{-} \pi^{+} \pi^{+}$ or $\overline{\mathrm{K}}^{\circ} \mathrm{o}^{+}$.

To the contrary, Ellis et al find $\Gamma\left(D^{+}+\overline{\mathrm{K}}^{\circ} \pi^{+}\right) \approx \Gamma\left(D^{\circ} \rightarrow \mathrm{K}^{-} \pi^{+}\right)$, a "sextetenhanced" rate. Using a variety of techniques, they estimate the following ranges of branching ratios for charmed-meson decay:

$$
\begin{aligned}
& \mathrm{B}\left(\mathrm{D}, \mathrm{F} \rightarrow \ell+v_{\ell}+\text { hadrons }\right)=0.1-0.25 \\
& \mathrm{~B}\left(\mathrm{D} \rightarrow \ell+v_{\ell}+\mathrm{K}\right)=0.03-0.08 \\
& \mathrm{~B}\left(\mathrm{~F} \rightarrow \ell+v_{\ell}+\eta\right)=0.02-0.05 \\
& \mathrm{~B}\left(\mathrm{D}^{\circ} \rightarrow \mathrm{K}^{-} \pi^{+}+\overline{\mathrm{K}}^{\circ} \pi^{\circ}\right)=0.03-0.18 \\
& \mathrm{~B}\left(\mathrm{D}^{+} \rightarrow \overline{\mathrm{K}}^{\circ} \pi^{+}\right)=0.02-0.10 \\
& \mathrm{~B}\left(\mathrm{~F}^{+} \rightarrow \mathrm{n \pi}^{+}+\mathrm{K}^{+} \overline{\mathrm{K}}^{\circ}\right)=0.02-0.12 .
\end{aligned}
$$


Arguments over operator enhancement aside, a11 authors (112-114) agree that, because of the large number of modes available for decay, no single branching ratio is expected to be more than a few percent.

One other interesting aspect of D-decays has to do with the possibility of $\mathrm{D}^{\mathrm{O}}-\overline{\mathrm{D}}^{\circ}$ mixing (115). If this is induced by charm-changing neutral currents such as $\bar{c}_{\gamma_{\lambda}}\left(\frac{1 \pm Y_{5}}{2}\right) u$ coupled to the $Z^{\circ}$ weak boson, then

$$
\Delta M\left(D^{0}, \bar{D}^{\circ}\right) \sim G_{F} M_{D}^{2}>\Gamma\left(D^{0} \rightarrow A L L\right) \sim G_{F}^{2} M_{D}^{5}
$$

and $D^{\circ} \bar{D}^{\circ}$ mixing will be complete. One then will see $D^{\circ} \rightarrow K+\ldots$ as often as $D^{\circ} \rightarrow \overline{\mathrm{K}}+\ldots$ If $|\Delta C|=2$ transitions are mediated by second order $\left(G_{F}^{2}\right)$ processes or by neutral Higgs bosons, mixing may be less than complete but still appreciable. Thus, a measurement of $\Gamma\left(D^{\circ}+\mathrm{K}+\ldots\right) / \Gamma\left(D^{\circ}+\overline{\mathrm{K}}+\ldots\right)$ gives us important information about the structure of weak currents (both charged and neutral) as well as constraints on the couplings of Higgs mesons to quarks.

Charmed baryon decays are considerably more complicated and correspondingly uncertain. The reader is referred to the above papers (and enclosed references) for details beyond the gross estimates one can make from Eqs. (4.6) and (4.7). As we hinted earlier, the masses of $D^{\prime} s$ and $D^{*}$ 's are so delicately arranged that they cause an unprecedented complication in sorting out D-spectroscopy. The problem is that the Q-values for $D^{*} \rightarrow$ Dm are so small that the electromagnetic (Ml) decay $\mathrm{D}^{*} \rightarrow \mathrm{D} \gamma$ is competitive with the strong one. Therefore, when studying the invariant mass recoiling against $\mathrm{D}^{\circ}$ produced in $\mathrm{e}^{+} \mathrm{e}^{-}$annihilation. at $4.028 \mathrm{GeV}$, say, one sees a very rich structure corresponding to

$$
\begin{array}{ll}
\mathrm{e}^{+} \mathrm{e}^{-} \rightarrow \mathrm{D}^{0} \overline{\mathrm{D}}^{\mathrm{O}} & \left(\text { Recoil mass } \mathrm{M}_{\mathrm{X}}=\mathrm{M}_{\bar{D}^{0}}\right) \\
\mathrm{e}^{+} \mathrm{e}^{-} \rightarrow \mathrm{D}^{\circ} \overline{\mathrm{D}}^{*_{0}} & \left(\mathrm{M}_{\mathrm{X}}=\mathrm{M}_{\bar{D}}^{-*_{0}}\right)
\end{array}
$$




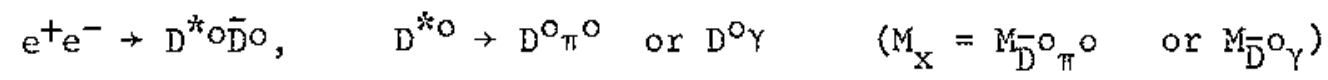

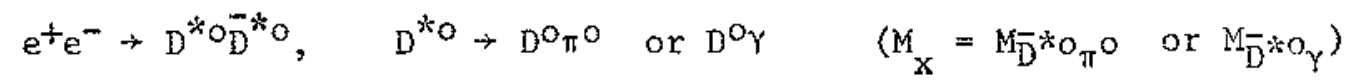

$$
\begin{aligned}
& \mathrm{e}^{+} \mathrm{e}^{-} \rightarrow \mathrm{D}^{*+\mathrm{D}^{-}}, \quad \mathrm{D}^{*+}+\mathrm{D}^{\mathrm{O} \pi^{+}} \quad\left(\mathrm{H}_{\mathrm{X}}=\mathrm{M}_{\mathrm{D}^{-} \pi^{+}}\right) \\
& \mathrm{e}^{+} \mathrm{e}^{-} \rightarrow \mathrm{D}^{*+} \mathrm{D}^{*-}, \quad \mathrm{D}^{*+} \rightarrow \mathrm{D}^{\mathrm{O}} \pi^{+} \quad\left(\mathrm{M}_{\mathrm{X}}=\mathrm{M}_{\mathrm{D}^{*-} \pi^{+}}\right)^{+}
\end{aligned}
$$

The decay $\mathrm{D}^{* 0} \rightarrow \mathrm{D}^{\mathrm{t}^{-}}$is energeticaliy forbidden. The relative strength of each component of the recoil distribution is determined by the product of the exclusive channel cross section and the appropriate $D^{*}$ branching ratio. So these branching ratios are of great importance in charmed-meson spectroscopy.

In addition to their model for calculating exclusive channel cross sections, Eichten et al (68) have estimated the various $D^{*}$ branching ratios as follows: For the Ml decays they use the naive quark model formula

$$
\Gamma\left(D^{*}(c \bar{q}) \rightarrow D(c \bar{q})+\gamma\right)=\frac{4}{3} \alpha\left(\frac{e_{c}}{2 m_{c}}+\frac{e_{q}}{2 m_{q}}\right)^{2} p^{3}
$$

where $e_{c}=e_{u}=2 / 3, e_{d}=-1 / 3, p=\left(M_{D}^{2} *-M_{D}^{2}\right) / 2 M_{D^{*}}$, and they use quark masses of $\mathrm{m}_{\mathrm{u}}=\mathrm{m}_{\mathrm{d}}=0.33 \mathrm{GeV}$ and $\mathrm{m}_{c}=1.87 \mathrm{GeV}$ (determined from $\Gamma\left(\psi \rightarrow \mathrm{e}^{+} \mathrm{e}^{-}\right.$) and $M_{\psi},-M_{\psi}$ in the pure linear potential model).

The $\mathrm{D}^{*} \rightarrow \mathrm{D} \pi$ width is obtained by assuming a form suggested by their mode1 calculation of $\psi_{\mathrm{nL}} \rightarrow \mathrm{DD}$; it is

$$
\Gamma\left(D^{*} \rightarrow D \pi\right)=\frac{\mathrm{p}^{3}}{72 \pi \mathrm{M}_{\mathrm{D}}^{2}} C^{2}\left|\sqrt{\mathrm{M}_{\mathrm{D}}^{*} \mathrm{E}_{\mathrm{D}} \mathrm{E}_{\pi}} \mathrm{A}\right|^{2}
$$

where $E_{D, \pi}=\sqrt{\mathrm{P}^{2}+\mathrm{M}_{\mathrm{D}, \pi}^{2}}, C$ is anisospin Clebsch-Gordan coefficient, and $A$ is an amplitude depending only on $\mathrm{m}_{\mathrm{u}}$ in the limit that heavy quark mass $\mathrm{m}_{\mathrm{c}} \rightarrow \infty$. Assuming further that $m_{s}$ is large enough so that A can be estimated from $k^{*} \rightarrow k \pi$ decays gives

$$
\mathrm{A}=47.8 \mathrm{GeV}^{-3 / 2}
$$


Using the measured ( 108 ) D and $D^{*}$ masses, they obtain the widths and branching ratios listed in Table 11 . As we will see shortly, the results in Table $1 \overrightarrow{1}$ are in remarkable agreement with those determined from experiment under much less model-dependent assumptions.

To conclude this discussion, we mention first that the expected (and measured) $F^{*}-F$ mass difference $\left(\leq M_{\pi}\right)$ implies the $F^{*} \rightarrow F \gamma$ is the only decay mode of this $C=S=1$ vector meson. Using $m_{s}=0.50 \mathrm{GeV}$ in Eq. (4.11). gives

$$
\Gamma\left(F^{*+} \rightarrow F Y\right)=0.2 \mathrm{keV}
$$

Second, the apparent success of the M1 formula Eq. (4.11) for D* decays stands in sharp contrast to its apparent failure in the charmonium system.

Finally, it is unfortunate that the formula Eq. (4.12) is unlikely to be testable in bound systems of a still heavier quark $Q$ with $u, d, s$. With the hyperfine splitting decreasing like $\mathrm{M}_{\mathrm{Q}}{ }^{-1}$, the only energetically allowed decays of $\mathrm{M}^{*}(\mathrm{Q} \overline{\mathrm{u}})$ to $\mathrm{M}(\mathrm{Qu})$, will be the radiative ones. Looking on the bright side, this situation will make $M, M^{*}$ spectroscopy a little easier, and-if the $\mathrm{M}^{*}$ width can be measured-provide further tests of the M1 formula.

\section{B. EXPERIMENT}

What follows is a brief discussion of the properties sumarized in Table 10 . For more detail and reference to experimental work not cited explicitly, the reader is referred to Feldman's recent review (108).

To date, charmed mesons have been identified directly only in $e^{+} e^{-}$annihilation experiments, where their production cross sections are $250 \%$ of the total and the kinematics are especially simple. The $D$ and $D^{*}$ mesons have been positively identified by the SLAC-LBL collaboration (22). We hasten to add that before and since their discovery, there has been plenty of indirect evidence for charmed mesons in neutrino experiments (see Sec. VI), in $\mathrm{e}^{+} \mathrm{e}^{-}$annihilation at 


\section{Table 11}

Predicted $\mathrm{D}^{*}$ Widths and Branching Ratios (68)

\begin{tabular}{|c|c|c|}
\hline Mode & Width (keV) & $\begin{array}{c}\text { Branching Ratio } \\
(\%)\end{array}$ \\
\hline$D^{* 0} \rightarrow D^{0} \pi^{\circ}$ & 39.7 & 53.0 \\
\hline $\mathrm{D}^{* \mathrm{O}} \rightarrow \mathrm{D}^{\mathrm{O}} \gamma$ & 35.2 & 47.0 \\
\hline$D^{*_{0}} \rightarrow a 11$ & 74.9 & \\
\hline $\mathrm{D}^{*+} \rightarrow \mathrm{D}^{+} \pi^{\circ}$ & 22.2 & 28.4 \\
\hline $\mathrm{D}^{*+} \rightarrow \mathrm{D}_{\pi}^{0+}$ & 53.4 & 68.4 \\
\hline$D^{*+} \rightarrow D^{+} \gamma$ & 2.5 & 3.2 \\
\hline $\mathrm{D}^{*+} \rightarrow \mathrm{a} 11$ & 78.0 & \\
\hline
\end{tabular}


CEA (14) SLAC (31, 32) and DESY $(33,34)$, and in photoproduction at SLAC (see Sec. VIC). The $\mathrm{F}^{+}$and $\mathrm{F}^{*+}$ (decaying to $\mathrm{F}^{+} \mathrm{Y}$ ) were discovered by the DASP collaboration (116) at DESY at c.m. energy $4.414 \mathrm{GeV}$. And the $\mathrm{F}^{+}$is tentatively confirmed by the SLAC-LBL collaboration in data taken at $4.16 \mathrm{GeV}$ (108). A11 of these discoveries and all of the precision data on $\mathrm{D}^{\circ}$ and $\mathrm{D}^{+}$were obtained at the peaks in the annihilation cross section-3.772, 4.028, 4.16, and 4.414 GeV-which are charmonium resonances above threshold. [There is one exception: the beautiful measurement (108) of $\mathrm{M}_{\mathrm{D}^{*+}}-\mathrm{M}_{\mathrm{D}^{\circ}}$ required high energy, $6.8 \mathrm{GeV}$, to detect the $\pi^{+}$in $\left.\mathrm{D}^{*+}+\mathrm{D}^{\mathrm{O}} \pi^{+}.\right]$

Evidence for charmed baryons comes from two sources. The first is a single neutrino event in the Brookhaven 7-foot bubble chamber (117).

$$
\nu_{\mu} \mathrm{p} \rightarrow \mu^{-} \mathrm{Am}^{+} \pi^{+} \pi^{+} \pi^{-}
$$

This is interpreted as $\nu_{\mu} \mathrm{p} \rightarrow \mu^{-} \Sigma_{\mathrm{c}}^{*++} ; \Sigma_{\mathrm{c}}^{*++} \rightarrow \Lambda_{\mathrm{C}^{+}}^{+} ; \Lambda_{\mathrm{C}}^{+} \rightarrow \Lambda^{+} \pi^{+} \pi^{-}$because the event violates the $\Delta S=\Delta Q$ rule. The second comes from a peak in the inclusive $\bar{A}^{-} \pi^{\sim} \pi^{+}$spectrum at $2.26 \mathrm{GeV}$ in a photoproduction experiment at Fermilab (118). This group also reports evidence for the sequence $\overline{\Sigma_{c}^{*_{0}}} \rightarrow \Lambda_{c}^{-} \pi^{+}$, with a $\Sigma_{c}^{*}$ mass of $2.43 \mathrm{GeV}$. These masses are exactly those determined in the Brookhaven experiment and expected in the quark model. Very indirect evidence for charmed baryons in $\mathrm{e}^{+} \mathrm{e}^{-}$annihilation comes from the sharp rise in proton and $\Lambda$ production in the $4.4-5.0 \mathrm{GeV}$ region (108). But upper limits on cross section times branching ratio to observable modes $(\sigma \cdot B)$ are typically an order of magnitude lower than for $D$ production at the same energies. Most interesting in these studies is that $A$ production is consistently on1y $10-15 \%$ of proton production at all energies, suggesting that charmed baryons preferentially decay to $\mathrm{K}+$ nucleon $+\ldots$ rather than to strange baryons.

The isospin assignments in the table are made purely on theoretical grounds; no experimental information exists other than the fact that $D^{*} \rightarrow$ D $\pi$ precludes 
$I=0$ for both these charmed mesons. ${ }^{15}$ similarly, no measurements of $J^{P}$ exist for $F, F^{*}$, and charmed baryons. Assuming that $D^{\circ}$ and $D^{+}$have the same $J^{P}$, observation of $\mathrm{D}^{\circ} \rightarrow \mathrm{K}^{-} \pi^{+}$and study of the Dalitz plot for $\mathrm{D}^{+} \rightarrow \mathrm{K}^{-} \pi^{+} \pi^{+}$proves that parity is violated in their decays (119), suggesting that they decay via weak interactions. This fact of parity violation is now obvious (without the assumption of equal $\mathrm{J}^{\mathrm{P}}$ ) from the observed decay modes of $\mathrm{D}^{\circ}$ and $\mathrm{D}^{+}$. Assuming that the parity of $D$ is -1 , observing that $D^{*}+D \pi$ and $e^{+} e^{-} \rightarrow D^{-*}$, and measuring the angular distributions for

$$
\mathrm{e}^{+} \mathrm{e}^{-} \rightarrow \underset{\mathrm{D}}{\overline{\mathrm{D}}} \longrightarrow \mathrm{K \pi}
$$

the SLAC-LBL collaboration is able to rule out $J_{D}^{P}=J_{D^{*}}^{P}$ for $J_{D}^{P}=0^{ \pm}$and $J_{D}^{P}=I^{-}, J_{D}^{P}=0^{-}$, whereas they find high confidence levels for the hypothesis $\mathrm{J}_{\mathrm{D}}^{\mathrm{P}}=0^{-}, \mathrm{J}_{\mathrm{D}^{*}}^{\mathrm{P}}=1^{-}$.

The remarkably precise measurements (108) of the $\mathrm{D}^{\circ}$ and $\mathrm{D}^{+}$masses come from data taken at the peak of $\psi^{\prime \prime}=\psi(3772)$, the $1^{3} \mathrm{D}_{1}$ charmonium level. This accuracy is possible because: (I) $\psi^{\prime \prime}$ decays exclusively to $\mathrm{D}^{\circ} \overline{\mathrm{D}}^{\circ}$ and $\mathrm{D}^{+} \mathrm{D}^{-}$(DD $\overline{\mathrm{D}}^{*}$ is energetically forbidden), and (2) it lies just $240 \mathrm{MeV}$ above threshold, so that the $D^{\prime}$ s are moving very slowly. Thus, small errors in the measurement of $\mathrm{P}_{\mathrm{D}}$ are unimportant in determining $\mathrm{M}_{\mathrm{D}}$ from

$$
M_{D}=\left(\left(M_{\psi} " / 2\right)^{2}-p_{D}^{2}\right)^{\frac{1}{2}}=\left(E_{b}^{2}-p_{D}^{2}\right)^{\frac{1}{2}},
$$

where the beam energy $E_{b}$ is very well measured. $p_{D}$, of course, is determined from the momenta of the D-decay products. Fig. 16 shows the invariant mass spectra for $D^{\circ}$ and $D^{+}$. The clearly visible $\delta=\mathrm{M}_{\mathrm{D}^{+}}-\mathrm{M}_{\mathrm{D}^{\circ}}=5.0 \pm 0.8 \mathrm{MeV}$ is only slightly less than predicted in Refs. 109 and 110.

The $D^{*}$ mass is measured by a similar trick at $W=4.028 \mathrm{GeV}$. Here, $\mathrm{e}^{+} \mathrm{e}^{-}+\mathrm{D}^{* 0} \mathrm{D}^{* 0}$ is picked out and $\mathrm{p}_{\mathrm{D}}$ \%o $_{0}$ is measured as follows: The momentum spectrum of $D^{\circ}$ 's detected at $\psi(4.028)$ is measured. (This is equivalent to the 
recoil mass spectrum, but is less susceptible to experimental error.) This is shown in Fig.17. The small Q-value for $D^{* O} \rightarrow D^{\circ}$. makes these components of the distribution (curves $B$ and $E$ in the figure) rather sharply peaked. The lower peak (B) obviously corresponds to $\mathrm{D}^{*} \mathrm{O}-\bar{D}^{*} \mathrm{O}$, and a simple kinematical exercise gives the center of this peak as

$$
\mathrm{P}_{\mathrm{D}}^{\mathrm{Ctr}}\left(\mathrm{D}^{*} \mathrm{O}^{-*} \mathrm{O} ; \mathrm{D}^{*} \mathrm{O} \rightarrow \mathrm{D}_{\pi}^{\circ}\right)=\mathrm{P}_{\mathrm{D} * \mathrm{O}^{\mathrm{E}}} \mathrm{D}^{\mathrm{O}} / \mathrm{M}_{\mathrm{D}}^{* O}
$$

Here, $E_{D^{\circ}}=M_{D}$ and $M_{D^{*}}=W / 2$ to a very good approximation, so that

$$
\mathrm{M}_{\mathrm{D} *_{0}}=\sqrt{(w / 2)^{2}-\left(\mathrm{p}_{\mathrm{D}}^{\mathrm{ctr}}\right)^{2}}
$$

determines the mass quite accurately. With the $D^{*+}-D^{\circ}$ mass difference accurately determined from high-energy data as noted above, there results $\delta^{*}=M_{D}{ }^{*+}-M_{D} *_{0}=2.6 \pm 1.8 \mathrm{MeV}$, and

$$
\delta-\delta^{*}=2.4 \pm 2.4 \mathrm{MeV}
$$

This is purely an electromagnetic hyperfine splitting and is expected to be $\checkmark 1 \mathrm{hfeV}$ in most theoretical estimates. Finally, the Q-values used in constructional Table 11 are shown in Fig. 18 .

Masses of the other charmed mesons are determined by similar techniques, with the most precise measurements always coming from $e^{+} e^{-} \rightarrow M_{c} \bar{M}_{c}$ where $M_{C}$ is a charmed meson, $\bar{M}_{c}$ its antiparticle. These masses are determined in standard ways dictated by the experimental set-up. And, finally, at $W=4.4$ $\mathrm{GeV}$, there is some evidence for peaking in the recoil mass distribution against $D^{\circ}$ (99). The peak occurs near $M_{x}=2.4 \mathrm{GeV}$, possibly corresponding to the quasi-two-body production of charmed P-states with $\mathrm{D}$ or $\mathrm{D}^{*}$. If we use the masses in Eq. (4.4), the process is

$$
e^{+} e^{-} \rightarrow D \bar{D}\left(I^{1} P_{I}\right)+D\left(I^{1} P_{I}\right) \bar{D}
$$


Before the discovery of $\psi^{\prime \prime}$, it was possible to measure with certainty on1y $\sigma \cdot B$ for the various $D$-meson decay modes. With this pure source of $\mathrm{D}^{\circ} \bar{D}^{\circ}$ and $\mathrm{D}^{+} \mathrm{D}^{-}$, comes the ability to measure absolute branching fractions. This, in turn, permits absolute determination of the charmed component of the total annihilation cross section. The main features of the nonleptonic fracm tions in Table 10 are: (1) they are a11 only a few percent, as expected; (2) the decay $\mathrm{D}^{+} \rightarrow \mathrm{K}_{\pi}{ }^{+}$does not seem suppressed, so that the nonleptonic $\Delta \mathrm{C}=\Delta \mathrm{S}$ Hamiltonian may not be as simple (or as mysterious-depending on one's point of view) as the one governing $\mathrm{K}$ decay; and (3) so far there is little evidence (119) for quasi-two-body decay, e.g. $\mathrm{D}^{\circ} \rightarrow\left(\mathrm{K}^{{ }^{*}-\pi^{+}}, \overline{\mathrm{K}}_{\rho}^{\circ}{ }^{\circ}\right) \rightarrow \overline{\mathrm{K}}^{\circ} \mathrm{O}^{+} \pi^{-}$. There is no evidence for Cabibbo-suppressed decays; present limits are somewhat above theoretica1 expectations of order $\tan ^{2} \theta_{c}$ (59).

The semielectronic decay fractions measured to date are really an average determined from measurement of

$$
\sum_{M_{C}=D^{\circ}, D^{+}, F^{+}, \ldots} \sigma\left(e^{+} e^{-}+M_{C}+\ldots\right) B\left(M_{c}+e^{+} \nu_{e}+\ldots\right)
$$

To reduce contamination from $F^{\prime} s$ and charmed baryons (so that their semileptonic fractions can be unfolded in future experiments), one wants data taken at the lowest possible energies. This still gives an average over $D^{\circ}$ and $D^{+}$, a problem that can be resolved using "tagged" D's from $\psi$ " decays (108). At any rate, three experiments have now measured the average semielectronic branching ratio at low energies: the DASP collaboration at DESY finds $\left\langle\mathrm{B}_{\mathrm{e}}\right\rangle=0.10 \pm 0.03$ at $\mathrm{W}=$ 3.99 - $4.08 \mathrm{GeV}(120)$; a LBL-SLAC-Stanford-Northwestern-Hawaii (LSSNH) collaboration gets $\left\langle\mathrm{B}_{e}\right\rangle=0.072 \pm 0.028$ from runing at $\psi^{\prime \prime}(121)$; and, at the same energy, the DELCO collaboration at SLAC has measured $0.11 \pm 0.03$ (122). It is worth remarking that DELCO has an order of magnitude more solid angle (60\%) for electron detection 
than do either of the other two experiments. The average of these three is

$$
\left\langle\mathrm{B}_{\mathrm{e}}\right\rangle=0.093 \pm 0.017
$$

In Table 10 we arbitrarily took $B_{e} \approx 10 \%$ for both $D^{\circ}$ and $D^{+}$.

This result is a factor of two smaller than expected in a naive quark-model. calculation, but within the wide range estimated by Ellis et al. (113).

The DASP, LSSNH, and DELCO collaborations also have measured the electron momentum spectrum in multiparticle events, presumably corresponding to $\mathrm{D} \rightarrow \mathrm{e}^{ \pm}+\mathrm{X}$ (rather than $\tau \rightarrow \mathrm{e}+\mathrm{X}$ ). Fig. 19 shows the DELCO results which have by far the highest statistics; the results of the other two groups are quite similar. Al1 of the spectra show the characteristic softness expected from $\mathrm{D} \rightarrow \mathrm{K}$ ev and $\mathrm{K}^{*} \mathrm{ev}$, with good fits obtained by assuming sizable fractions of these two modes. None of the experiments was sensitive to the $\mathrm{V}$, A structure of $\mathrm{D} \rightarrow \mathrm{K}^{*}$ ev. Certainly, this is one of the most important questions for future study.

The question of $D^{\circ}-\vec{D}^{\circ}$ mixing has been studied by two methods. The first (99) is to observe $\mathrm{D}^{\mathrm{O}} \rightarrow \mathrm{K}^{-} \pi^{+}$and look at the charge of the kaon resulting from decay of the $\bar{D}$ in recoil. The second (108) is to tag $D^{\circ}$ by observing the $\pi^{+}$in $\mathrm{D}^{*+} \rightarrow \mathrm{D}^{\mathrm{O}} \pi^{+}$and then count the number of times $\mathrm{D}^{\circ} \rightarrow \mathrm{K}^{+} \pi^{-}$instead of $\mathrm{K}^{-} \pi^{+}$. In both cases, the apparent $\Delta \mathrm{C}=-\Delta \mathrm{S}$ decays are consistent with what is expected from $\pi-K$ misidentifications; and the violation of the $\Delta C=\Delta S$ rule is $\leq 17 \%$ at the $90 \%$ confidence level. This certainly rules out maximal $D^{\circ}-\overline{\mathrm{D}}^{\circ}$ mixing, i.e. $|\Delta C|=2$ currents coupled to $z^{\circ}$, but not necessarily a sma11 mixing due to second-order weak or Higgs boson effects.

$D^{*}$ branching ratios may be determined as follows (108): Fitting the relative contributions of curves $B$ and $C$ in Fig. 17, there results (99)

$$
B\left(D^{* O} \rightarrow D^{\circ} Y\right)=0.45 \pm 0.15
$$


Hence, $B\left(D^{* 0} \rightarrow D^{\circ} \pi^{\circ}\right)=0.55 \pm 0.15$, and $D^{*+}$ branching ratios may be obtained under more general assumptions than made in Eq. (4.12), namely (1) isospin conservation in $\mathrm{D}^{*} \rightarrow \mathrm{D} \pi$ decays; (2) $\Gamma\left(\mathrm{D}^{*} \rightarrow \mathrm{D} \pi\right)$ proportional to $\mathrm{P}_{\mathrm{D}}^{3}$; (3) Eq. (4.11) for $\mathrm{M} 1$ rates. It is then clear that if $I=1 / 2$ for $D$ and $D^{*}$ and $m_{1} / m_{c} \leqslant 1 / 4$, the resulting widths and branching franctions are nearly indistinguishable from the predicted ones in Table 11 .

All in all, the gross characteristics of charmed hadrons are just what were expected on theoretical grounds. But the theory is a long way from being welltested, and a great deal more experimental study of the details of charmed-particle weak interactions is needed. It is to be hoped that this "bread-and butter" physics (best carried out at the $\psi "$ ) will not be overlooked in the rush for new physics at the $T$ and still higher energies. 


\section{$\checkmark$ BEYOND CHARM}

\section{A. MOTIVATIONS}

- The existence and characteristics of the quarks $u, d, s$ and $c$ are well-established. However, there are reasons for considering the existence of further quarks, even heavier than the $\mathrm{c}$. The quarks to be considered here are those of charga $\frac{2}{3}$ (called $t$ quarks) and of charge $-\frac{1}{3}$ (called b quarks). While there is no known reason that quarks of charges $-\frac{4}{3},+\frac{5}{3}$ (etc.) are forbidden, there is no theoretical or experimental motivation for them; they present no essentially different features and will not be discussed.

The theoretical motivation for quarks beyond charm comes from gauge theories for the weak and electromagnetic interactions. Within the group su(2) $\times U(1)$ there is little purely theoretical motivation for more than four quarks. However, if for aesthetic or other reasons, one required the existence of both left- and righthanded charged currents (for quarks), then no $S U(2) \times U(1)$ model with only four quarks is even remotely consistent with the data. One six-quark model (123) with the coupling ${ }^{16}(\mathrm{u} b)_{R}$ (and $m_{b}>11$ or $12 \mathrm{GeV}$ ) is possible; all other $\mathrm{SU}(2) \times \mathrm{U}(1)$ models (124) with right-handed couplings for u and/or d quarks appear to be inconsistent with the data. Of course, there is absolutely no experimental evidence requiring any right-handed charged currents among quarks.

Many models based on higher gauge groups require six (or more) quarks. If an Su(3) of "flavor" is contained in the group, then triplets of quarks with charges $\frac{2}{3},-\frac{1}{3},-\frac{1}{3}$ can be found. 17 Some argue that the non-integer nature of quarks can be understood in a natural manner in such theories where the sum of quark charges is zero. In theories involving SU(3), the fifth and sixth quarks are both expected to have charges $-\frac{1}{3}$. It should be mentioned that some higher groups contain $\mathrm{SU}(2) \times \mathrm{U}(1)$ as a physically relevant subgroup and are able to reproduce all of 
the WS-GIM predictions for neutrino-hadron interactions. SU(3) generalizations of $\mathrm{SU}(2) \times \mathrm{U}(1)$ models will be discussed in Sec. V B.

There have been attempts to find theories which unify the strong interactions with the electromagnetic and weak interactions. Among such theories are those based on the exceptional groups $E_{6}$ and $E_{7}$, studied by Gürsey, Ramond and Sikivie (125). The group $\mathrm{E}_{6}$ has $\mathrm{SU}(3) \times \mathrm{SU}(3) \times \mathrm{SU}(3)^{\text {color }}$ as a maximal compact subgroup, while $\mathrm{E}_{7}$ has $\mathrm{SU}(6) \times \mathrm{SU}(3)^{\text {color }}$. The $\mathrm{E}_{6}$ theory can be reduced to a model very similar to the WS-GIM model, and $E_{7}$ to the $\mathrm{SU}(2) \times \mathrm{U}(1)$ model with (u $\left.\mathrm{b}\right)_{\mathrm{R}}$. Both models require six quarks (two with charges $\frac{2}{3}$ and four with $-\frac{1}{3}$ ). The quarks found in these theories based on exceptional groups automaticaIly have fractional charges.

There are further motivations for new quarks. Following the discovery of the charmed quark, four quarks and four leptons were known to "exist." Later a fifth lepton $\tau$ (of mass $1.8 \mathrm{GeV}$ and charge \pm 1 ) was discovered (24). Horn and Ross (126) showed that in the WS-GIM model, existing data require the existence of a neutral lepton coupled to $\tau$ (as $v_{e}$ is to e). Some have speculated that these additional leptons may indicate the need for additional quarks. Within the WS-GIM model, it is necessary to have equal numbers of quark doublets and lepton doublets in order to cancel WA triangle anomalies (127) which otherwise prevent renormalizability of the gauge theory. In any unified gauge theory, the proof of renormalizability makes strong use of current conservation through the associated Ward identities (9). The formal conservation of axial currents, however, is not necessarily true in the presence of fermions. Triangle diagrams with one axial and two vector vertices will destroy the axial conservation unless cancel.lations are arranged among the different fermions which can circulate in the loop. In the WS-GIM model, the anomaly cancels between the ( $\left.v_{e} e\right)_{L}$ and (u d) ${ }_{L}$ doublets, 
and between the $\left(v_{\mu} \mu\right)_{L}$ and $(c s)_{L}$ doublets. Therefore the presence of a doublet associated with $\tau$ requires a quark doublet ( $t$ b) ${ }_{L}$ in that model. It may be relevant to metion that the present, limited data shows the branching ratio (128) for the decay $\tau \rightarrow v \pi$ to be substantially lower than expected; this apparent failure of a firm theoretical prediction clouds the interpretation of t, but more data is needed before taking this result seriously. In other models the triangle anomalies are cancelled by diffexent means so that conclusions concerning new quarks can be different.

An early experimental motivation for new quarks was the report of anomalous energy dependencies for antineutrino scattering cross-sections and distributions (129). However, more recent experiments (130) with higher statistics find no large anomalies. Another relevant observation in neutrino scattering has been the discovery (131) of events with three outgoing muons $\left(\mu^{-} \mu^{-} \mu^{+}\right.$). The number of events reported at this time is quite small, and it is impossible to determine their origin now. Three possible sources involving new heavy particles have been suggested; however, two "background" sources could also provide a significant rate of "trimuon" production. One source of trimuons in neutrino scattering could be the production of a charged heavy lepton (heavier than the $\mathrm{t}$ lepton) which has a sequential decay (involving another new heavy lepton) into three muons and other particles (132). Another source (133) involves the simultaneous production of a neutral jepton (which decays into $\mu^{-} \mu^{+} v$ ) and a quark b (which decays into a negative mon and other particles). Finally, a heavy quark $t$ could be produced and decay sequentially through either a quark b or a neutral heavy lepton (134). Alternatively, trimuon events could simply be the result of $\rho, \omega, \phi$ and $\psi$ production and decay to $\mu^{+} \mu^{-}$, or muon pair bremsstrahlung (135) off quarks or the muon. At this time one cannot, therefore, say whether or not trimuon events are an indication 
of the existence of new heavy quarks, but the amount of data should increase sharply in the near future.

There is, of course, one substantial motivation for quarks beyond charm. Upon its discovery ( 23$)$ in pp scattering, the $T(9.4)$ was immediately interpreted as a $\bar{q} q$ meson (103). Analyses indicated that the associated quark had charge $-\frac{1}{3}$; however, these analyses involve significant assumptions, and it should be emphasized that one cannot reach reliable conclusions concerning the charge in hadronic collisions.

In $\mathrm{e}^{+} \mathrm{e}^{-}$annihilation it should be easy to determine the nature of T (9.4) and the charge of its constituent quark. The charge will be evident by deternination of the leptonic width, $\Gamma\left(\mathrm{T} \rightarrow \mu^{+\mu^{-}}\right)$, (about $0.7 \mathrm{keV}$ for b quarks and $2.8 \mathrm{keV}$ for t quarks of mass $5 \mathrm{GeV}$ according to Eichten and Gottfried (104)). Use of the branching ratio of $\mathrm{T}^{*}$ to $\mu^{+} \mu^{-}$is not completely reliable, since theoretical calcuIation of the total width is difficult. The cross-sections expected for $\tilde{i}$ are much smaller than those for $\psi$ (136). The integrated area under a resonance in $\mathrm{e}^{+} \mathrm{e}^{-}$annihilation is given by

$$
\Sigma=\frac{6 \pi^{2}}{m_{r}^{2}} B_{\text {had }} \Gamma\left(\psi \rightarrow e^{+} e^{-}\right)
$$

where $\Gamma\left(\psi \rightarrow \mathrm{e}^{+} \mathrm{e}^{-}\right)=\Gamma\left(\psi \rightarrow \mu^{+} \mu^{-}\right)$. For a $-\frac{1}{3}$ charge quark one finds $\Sigma \approx 150 \mathrm{nb}-\mathrm{MeV}$ compared with $10^{4} \mathrm{nb}-\mathrm{MeV}$ for $\psi$, and the signal to background may be only 2 to 1. The maximum ratio of the cross-section in the resonance $\mathrm{T}$ (for charge $-\frac{1}{3}$ ) to background would be approximately 10 compared to 300 for $\psi$. While $\mathrm{T}$ will not be as dramatic as $\psi$, it will be quite noticeable in $\mathrm{e}^{+} \mathrm{e}^{-}$experiments, and its discovery there will be an important confirmation of a new quark. 


\section{B. EXTENDING THE STANDARD MODEL}

The simplest extension of the WS-GIM model within SU(2) $\times U(1)$ is the addition of a new Teft-handed doublet with $t$ and b quaxks (137), which, together with a new doublet for $\tau$ leptons, would cancel triangle anomalies:

$$
\begin{aligned}
& \left(\begin{array}{l}
u \\
d
\end{array}\right)_{L} \quad\left(\begin{array}{l}
c \\
s
\end{array}\right)_{L} \quad\left(\begin{array}{l}
t \\
b
\end{array}\right)_{L} \\
& \left(\begin{array}{c}
v_{e} \\
e
\end{array}\right)_{L} \quad\left(\begin{array}{c}
v_{H} \\
\mu
\end{array}\right)_{L} \quad\left(\begin{array}{c}
v_{\tau} \\
\tau
\end{array}\right)_{L}
\end{aligned}
$$

with all right-handed components in singlets. These new doublets have little impact on the phenomenology of the lighter particles. The $d$, $s$ and $b$ quarks in these doublets are actually mixtures similar to the Cabibbo mixture for the four-quark model (see the discussion in Sec. VIII). As discussed in Sec. VI and VII and elsewhere, there is almost no data in conflict with this expanded WS-GIM modeI.

Within the gauge group $\mathrm{SU}(2) \times \mathrm{U}(1)$ it is also possible to construct models with right-handed charged currents. The relevant couplings are those to u and d quarks. Some models $(123,124)$ have $(u b)_{R}$ or $(t d)_{R}$ or both. If one is willing to consider quarks of charge $-\frac{4}{3}$ or $+\frac{5}{3}$, then models with $(\mathrm{d} v)_{\mathrm{R}}$ or $(x \mathrm{u})_{\mathrm{R}}$ can be obtained. Of models with such right-handed doublets, only one (123) is consistent with present neutral-current data (see Sec. VII):

$$
\begin{aligned}
& \left(\begin{array}{l}
u \\
d
\end{array}\right)_{L}\left(\begin{array}{l}
c \\
s
\end{array}\right)_{L} \\
& \left(\begin{array}{l}
u \\
b
\end{array}\right)_{R} \quad\left(\begin{array}{l}
c \\
g
\end{array}\right)_{R} \\
& \left(\begin{array}{c}
\nu_{\mathrm{e}} \\
\mathrm{e}
\end{array}\right)_{\mathrm{L}}\left(\begin{array}{c}
\nu_{\mu} \\
\mu
\end{array}\right)_{\mathrm{L}} \quad\left(\begin{array}{c}
\nu_{\tau} \\
\tau
\end{array}\right)_{\mathrm{L}} \quad\left(\begin{array}{c}
\mathrm{N}_{\mathrm{e}} \\
\mathrm{e}
\end{array}\right)_{\mathrm{R}} \quad\left(\begin{array}{c}
\mathrm{N}_{\mu} \\
\mu
\end{array}\right)_{\mathrm{R}}\left(\begin{array}{c}
\mathrm{N}_{\tau} \\
\tau
\end{array}\right)_{\mathrm{R}}
\end{aligned}
$$

where $m_{b}>11$ or $12 \mathrm{GeV}$ (see Sec. VI) but the g quark could be the constituent of $\Upsilon$. $N_{e}, N_{y}$ and $N_{\tau}$ are heavy neutral leptons. For this model, the cancellation of 
triangle anomalies occurs separately within the quark sector and within the lepton sector.

One canmodify such models to include a coupling $(c s)_{R}$ but not $(c d){ }_{R}$; this has been discussed by Golowich and Holstein and othexs (138). There is no reason, a priori, that quarks (or leptons) must be in doublets. However, $S U(2)$ triplets (or higher representations) require quarks of charge $-\frac{4}{3}$ or $+\frac{5}{3}$, and will not be considered here.

We have mentioned a variety of theoretical reasons for considering other weak and electromagnetic gauge groups beyond $\mathrm{SU}(2) \times \mathrm{U}(1)$. Furthermore it is possible that future data will rule out $\mathrm{SU}(2) \times \mathrm{U}(1)$ as the full group. However the present phenomenological success of the WS-GIM mode1 indicates that $S U(2) \times U(1)$ wi.11 be a good subgroup of any larger group.

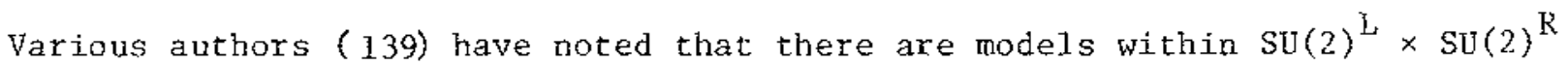
$\times U(1)$ (where $\mathrm{L}=1$ eft and $\mathrm{R}=$ right and $\mathrm{SU}(2)^{\mathrm{L}}$ is the same $\mathrm{SU}(2)$ as above) which reproduce virtually a11. the neutrino-hadron scattering results of the WS-GIM model. Georgi and Weinberg ( 140 ) have generalized these results and shown that at zeromomentum transfer, the neutral-current interactions of neutrinos in an $S U(2) \times G$ $\times U(1)$ gauge theory are the same as in the corresponding $S U(2) \times U(1)$ theory if neutrinos are neutral under $G$. They also noted that one of the neutral gauge bosons in the expanded group must have a mass below that of the $Z^{\circ}(80 \mathrm{GeV})$ of the $\mathrm{SU}(2) \times \mathrm{U}(1)$ model.

In $\operatorname{SU}(2)^{\mathrm{L}} \times \mathrm{SU}(2)^{\mathrm{R}} \times \mathrm{U}(1)$ there are seven gauge bosons, $W_{\mathrm{L}}^{ \pm}, W_{R}^{ \pm}, Z_{1}^{0}, Z_{2}^{0}, \gamma$, in contrast to the four in $S U(2) \times U(1)\left(W^{+}, Z^{0}, \gamma\right)$. It can be arranged so that $Z_{1}^{0}$ has purely axial-vector couplings to all particles (except neutrinos) and that $z_{2}^{0}$ has purely vector couplings; this assures the absence of parity violation in neutral-current interactions (see Sec. VII). One version of the model has the couplings: 


$$
\begin{aligned}
& \left(\begin{array}{l}
u \\
d
\end{array}\right)_{L}\left(\begin{array}{l}
c \\
s
\end{array}\right)_{L} \quad\left(\begin{array}{l}
t \\
b
\end{array}\right)_{L} \quad \begin{array}{llll}
(u b)_{R} & (c s)_{R} \quad(t ~ d) R
\end{array} \\
& \left.\left(\begin{array}{c}
v_{\mathrm{e}} \\
\mathrm{e}
\end{array}\right)_{\mathrm{L}}\left(\begin{array}{c}
v_{\mu} \\
\mu
\end{array}\right)_{\mathrm{L}}\left(\begin{array}{c}
v_{\tau} \\
\tau
\end{array}\right)_{\mathrm{L}} \quad\left(\mathrm{N}_{\mathrm{e}}\right)_{\mathrm{R}} \quad \mathrm{N}_{\mu} \mu\right)_{R} \quad{ }^{\left(\mathrm{N}_{\tau} \tau\right)_{R}}
\end{aligned}
$$

where column doublets are coupled by $W_{L}$ (the usual $W$ ) and row doublets by $W_{R}$. Since $(u b)_{R}$ is coupled by $W_{R}$, which has no direct couplings to $\nu_{\mu}$, the usual lower limits on the mass of $b$ do not apply (and $T \equiv \bar{b} b$ is possible).

Another gauge group which has received considerable attention is $\mathrm{SU}(3) \times \mathrm{U}(\mathrm{I})$. For the models (141) which have been considered, extreme values of the parameters can be chosen which would reduce these models to conventional $S U(2) \times U(1)$ models. For intermediate values of the parameters, the phenomenological results are somewhat different. One version of the models resembles the WS-GIM model, while another resembles the $\mathrm{SU}(2) \times \mathrm{U}(1)$ model with $(u b)_{R}$.

One extension $(125,142)$ of the standard WS-CIM model, which has SU(2) $\times U(1)$ as a good subgroup in a fairly natural way, is based on the group SU(3) $x$ SU(3). The neutrino-hadron scattering results are essentially the same as for the WS-GIM model although the value $\sin ^{2} \theta_{W}=3 / 8$ predicted in this model seems somewhat larger than present experimental indications. There are 16 gauge bosons, including the usual $W^{ \pm}, Z^{\circ}$ and $\gamma$. Many of these bosons must be three (or more) times as heavy as the $W^{ \pm}$for phenomenological purposes. Among these are the "right-handed" equivalents of $\mathrm{W}^{ \pm}$and most of the bosons carrying diagonal neutral-currents. In this model the leptons are placed in two $(\overrightarrow{3}, 3)$ representations. The quarks are in triplets such as:

$$
\left(\begin{array}{c}
u \\
d \\
b
\end{array}\right)_{L}\left(\begin{array}{l}
c \\
s \\
g
\end{array}\right)_{I_{t}} \quad\left(\begin{array}{lll}
u & s & b
\end{array}\right)_{R} \quad\left(\begin{array}{ccc}
c & g
\end{array}\right)_{R}
$$


where the first two quarks in each colum triplet are coupled by $w^{ \pm}$and all other quarks are coupled by different bosons. One of the most interesting features of this model is that the lightest new quark b, always decays semileptonically, including modes such as $b \rightarrow u \ell^{-} \bar{v}$ and $b \rightarrow d v_{\bar{v}}$. Thus, this model predicts a large amount of missing neutral energy in $\mathrm{e}^{+} \mathrm{e}^{-} \rightarrow(\mathrm{b} \overline{\mathrm{q}})+(\bar{b} \mathrm{q})$.

One of the questions in constructing new models concerns the weak coupling of the $b$ quark where $T(9.4) \equiv b \bar{b}$. While the standard assumption places the $b$ guark in a left-handed doublet with a $t$ quark, there are several other couplings which are consistent with all data (see secs. VI and VII). In SU(2) $x$ U(1) models, the couplings $(t b)_{R}$ and $(c b)_{R}$ are allowed. In a model such as $\mathrm{SU}(2)^{\mathrm{L}} \times \mathrm{SU}(2)^{\mathrm{R}} \times \mathrm{U}(1)$ the b quark can even have a right-handed coupling to u quarks since that interaction is mediated by $W_{R}$ which does not couple to $v_{\mu}$. Those models which have quarks in SU(3) triplets can have couplings such as (u d b $)_{L^{\prime}}\left(t \quad b\right.$ d) $R$ or (c b d) $R^{*}$ There certainly is no evidence that b quarks have left-handed couplings to $t$ quarks. In fact, sone of the modeis mentioned here have no $t$ quarks.

Not all theories involve quarks with fractional charge. Pat1, Salam and others (143) have proposed models with quarks of integer charge (following Han and Nambu ( 144 )), which nonetheless reproduce many of the results of conventional gauge theories. In this theory, however, quarks and gluons can exist as free particles (before decaying). In the basic mode1, there are 16 fermions:

$$
\left(\begin{array}{cccc}
c_{R} & u_{Y} & u_{B} & v_{e} \\
d_{R} & d_{Y} & d_{B} & e^{-} \\
s_{R} & s_{Y} & s_{B} & \mu^{-} \\
c_{R} & c_{Y} & c_{B} & v_{\mu}
\end{array}\right) \text { with charges }\left(\begin{array}{cccc}
0 & 1 & 1 & 0 \\
-1 & 0 & 0 & -1 \\
-1 & 0 & 0 & -1 \\
0 & 1 & 1 & 0
\end{array}\right)
$$

where $R, Y, B$ are the "colors" red, yellow, blue, and the leptons are considered to be the fourth color. The model can be expanded to include other quarks and 
leptons. One of the problems for this model is that it predicts free, massive gluons which have not been observed. This and other aspects of the Pati-Salam model are discussed critically in Ref. 145 . 


\section{PRODUCTION BY NEUTRINOS, HADRONS AND PHOTONS}

Although most experimental information about new quarks has come from $\mathrm{e}^{+} \mathrm{e}^{-}$annihilation, other methods of production have played an extremely important role. The $\Psi / J$ was produced hadronically at the same time that it appeared in $e^{+} e^{-}$annihilation, and charmed baryons have been observed only in neutrino and photoproduction. In this section, some of these other methods are discussed. They can yield important information about the weak and electromagnetic theory and about QCD as a possible strong interaction theory.

\section{A. PRODUCTION BY NEUTRINOS}

In neutrino scattering in the WS-GIM model, where u quarks have a left-handed coupling to d quarks ( $u d)_{L}$, one expects $\nu_{\mu} d \rightarrow \mu^{-} u$ or $\bar{v}_{\mu} u \rightarrow \mu^{+} d$ to be the usual charged-current processes. Most results are consistent with this hypothesis, and one must look at rare processes in order to learn more.

In the scattering of neutrinos off nucleons, it is possible to produce single charmed mesons (or baryons). However, since there is no large coupling of valence (u or d) quarks to $\mathrm{c}$ quarks, this additional cross-section is not large. The coupling $\left(\overline{\mathrm{c}} \mathrm{d} \sin \theta_{\mathrm{c}}\right)$ with $\sin ^{2} \theta_{\mathrm{c}} \approx 0.05$ leads to a $5 \%$ rise in the expected cross-section for neutrinos (above the threshold energy). There is no similar Cabibbo-suppressed $\left(\sin \theta_{\mathrm{c}}\right.$ ) process possible for antineutrinos. The coupling (cs $\cos \theta_{c}$ ) leads to an increase in both neutrino and antineutrino cross-sections; however, the amount of strange quarks in the sea (i.e., of $s-\bar{s}$ pairs in the nucleon) is quite sma11, of order $5 \%$ (146), so that resulting effects are small. Since $5 \%$ effects are difficult to measure experimentally and since comparable or larger QCD effects may occur, little evidence for chamn is found in total crosssections. Similarly, little effect is seen in $y$ distributions $\left(y \equiv\left(E_{\nu}-E_{\mu}\right) / E_{\nu}\right)$.

It might be helpful to give a simplified description of several features of QCD which should result in similar effects in neutrino scattering (147). With increasing $Q^{2}\left(\equiv-q^{2}\right)$ one expects that: a) the $x$ distributions of quarks (where 
$\mathrm{x} \equiv-\mathrm{q}^{2} / 2 \mathrm{~W}_{\mathrm{N}}\left(\mathrm{E}_{\nu}-\mathrm{E}_{\mu}\right)$ ) will shrink (i.e. - become more peaked toward zero); b) the fraction of the struck nucleon's momentum carried by valence quarks will decrease slowly; and c) the fraction carried by sea quarks ( $u-\bar{u}, d-\bar{d}$ and $s-\bar{s}$ pairs in the nucleon) will increase. There are helicity arguments which show that $\sigma\left(\mathrm{vq}_{1}+\mu^{-} q_{2}\right)$ $=3 \sigma\left(\nu \vec{q}_{2} \rightarrow \mu^{-} \vec{q}_{1}\right)=3 \sigma\left(\vec{v} q_{2} \rightarrow \mu^{+} q_{1}\right)=\sigma\left(\bar{v} \bar{q}_{1} \rightarrow \mu^{+} \bar{q}_{2}\right)$, where $\bar{q}$ indicates antiquark. In neutrino reactions then, scattering off valence quarks is enhanced relative to that off sea quarks, while in antineutrino reactions scattering off sea quarks is enhanced (although most momentum is always carried by valence quarks). As a result, one expects neutrino cross-sections to decrease with increasing $E_{\nu}$ (which is proportional to $\left\langle Q^{2}\right\rangle$ ) and antineutrino cross-sections to increase slight1y. A related effect is the increase of $\langle y\rangle$ for antineutrinos with increasing $E_{\nu}$ (for neutrinos there is little effect).

Although charm is difficult to detect in total cross-sections and distributions, evidence for charm is quite clear in other aspects of neutrino experiments. Charmed particles decay into muons and into electrons 10 or $20 \%$ of the time, and these leptons can be detected. If charm production is 5 or $10 \%$ of the total, and the branching ratio to muons (electrons) is $10 \%$ or $20 \%$, then $0.5 \%$ to $2 \%$ of al1 neutrino-induced events should contain an extra muon (electron). This rate of "dilepton" production (17) is in fact roughly what is observed (due to experimental cuts and efficiencies the exact rate is not easy to determine directly). Furthermore, in distributions of variables such as $y, E_{\mu}$ and various angles, one finds (148) strong evidence for the additional lepton coming from the decay of a produced heavy quark (with mass of approximately $2 \mathrm{GeV}$ ).

Neither the rate nor the distributions show that this heavy quark is charm. However, since charm usually decays to $s$ quarks, in bubble chamber experiments one can see if events with two leptons also have a $K$ meson or a $A^{\circ}$ baryon. When 
neutrinos change d quarks into c quarks, one strange farticle should result. However, when an 3 quall: in the sea is changed into a c quark, there is always the remaining $\bar{s}$ quark from the pair in addition to the s quark from $c$ quark decay, so that two strange particles result. Since antineutrino scattering lacks a Cabibbo-suppressed mode of charm production, the number of strange particles (two) is expected to be greater than for neutrinos (roughly 1.5). At present, results have been reported only for neutrinos. Two experiments (149) have reported about $3.5 \mathrm{~K}$ mesons per $\mu^{-} \mathrm{e}^{+}$event, while one other (150) with much higher statistics has reported about $1.0 \mathrm{~K}$ mesons per event. The 1atter corresponds closely to the predictions for charm.

In all of these features (cross-sections, y distributions, dilepton rates, presence of strange particles) little room remains for significant production of any heavier quarks. Of course, for sufficiently massive quarks, a11 production would be deferred until higher energies. Present data (129,130) indicate that any b quark (charge $-\frac{1}{3}$ ) which has a right-handed coupling to u quarks (through $W$ bosons) nust have $m_{b} \geq 11$ or $12 \mathrm{GeV}$, certainly excluding the quark in $\Upsilon(9.5)$. If given that $m_{b}=5 \mathrm{GeV}$, then the coupling squared for ( $\left.u b\right)_{R}$ must be 0.1 (or

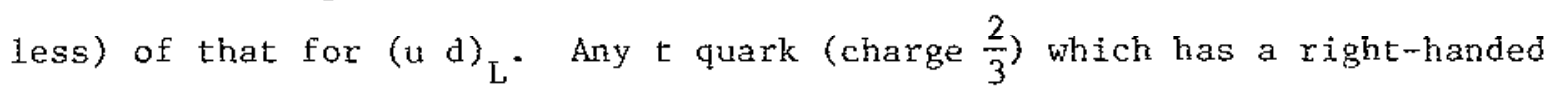
coupling to $d$ quarks (through $\mathrm{w}$ bosons) must have $\mathrm{m}_{t} \geq 5$ or $6 \mathrm{GeV}$. For the lefthanded couplings $(u b)_{L}$ and $(t d)_{L}$, the limits from analysis of the data are $\mathrm{m}>8 \mathrm{GeV}$ in both cases if the couplings are full strength. For $5 \mathrm{GeV} t$ or b quarks, the (left-handed) couplings squared must be 0.3 (or less) of that for $\left(\begin{array}{ll}\mathrm{d} & \mathrm{d}\end{array}{ }_{\mathrm{L}} \cdot\right.$

In the WS-GIM model, the additional coupling $(t \quad b)$ L (see Sec V) wovid lead to little $t$ or $b$ quark production, because the mixing angles between heavy quarks and 1ight quarks must be small (see Sec. VIrI A). From the universality of quark 
and lepton couplings and from the $\mathrm{K}_{\mathrm{L}}^{\circ}-\mathrm{K}_{\mathrm{S}}^{0}$ mass difference, one finds (151) that the $\bar{t}$ coupling ( $\overrightarrow{u b}$ coupling) is not likely to be more than $10 \%(5 \%)$ of the $\bar{u}$ d coupling (i.e. - rates at the $1 \%(0.3 \%)$ leve1). Clearly even at high energies there will be little impact on cross-sections.

There are many other couplings possible for $t$ and $b$ quarks besides $(t b)_{L}$, and some of these also have few observable consequences in neutrino physics. Among such couplings are the right-handed couplings ( $t b)_{R}$ and $\left(\begin{array}{cc}c & b\end{array}\right)_{R}$. Also the couplings $(u b)_{R}$ and $(t d)_{R}$ are possible (even for relatively light $t$ and $b$ ) in models such as $S U(2)^{\mathrm{L}} \times \mathrm{SU}(2)^{\mathrm{R}} \times U(1)$, where those couplings occur not through the usual $W$ boson but through a new boson which does not couple $\nu_{\mu}$ to $\mu$ and is heavier than $w$.

One can look for signals for new quarks in multi-lepton events, although in the WS-GIM model, the rates will not be high. Given the coupling squared $(\leq 0.01)$, the branching ratio to muons $(\sim 0.2)$ and the phase space suppression $\vec{F}$ (see Table 12), the rate for dilepton events from new heavy quarks is less than $0.2 F$ times the rate for dileptons from charm in this mode1. Clearly then, detection of $t$ and $b$ quarks will be difficult. One possible method of distinguishing dileptons for $t$ or $b$ decay from dileptons for charm decay involves the energy of the secondary (decay) lepton. Dileptons from heavy quark decay should be significantly more energetic (152). 


\section{Table 12}

The phase space suppression $F$ (relative to zero mass quarks) for production in neutrino scattering of quarks of given mass.

$$
\text { F for Fermilab }
$$

Quark Mass

$(\mathrm{GeV})$

5

10

15

Quad Triplet Flux

A11 E

$\mathrm{E}>100 \mathrm{GeV}$

$$
10^{-1}
$$$$
6 \times 10^{-3}
$$

$10^{-4}$
F for CERN

Wide-Band Flux

AlI E $\quad E>100 \mathrm{GeV}$

$10^{-1}$

$2 \times 10^{-1}$

$10^{-3} \cdot 10^{-2}$

$10^{-5}$

$10^{-4}$ 
Another means of finding evidence for the production of $t$ or $b$ quarks comes from examination of events in which three leptons are produced. In the WS-GIM model, $b$ quarks are likely to decay into c quarks unless $m_{t}<m_{b}$ (see Sec. VIII A). In some cases, both the $b$ and $c$ quark decays would involve leptons. In antineutrino scattering, $b$ quarks can then be produced (along with the usual $\mu^{+}$) and decay sequentially into two muons (or electrons). The resultant "trilepton" events would occur at less than $10^{-4} \mathrm{~F}$ (see Table 12 ) of the total rate. If $\mathrm{m}_{\mathrm{b}}>\mathrm{m}_{t}$, it is clear that trilepton events can also occur at the same rate, but with $b$ decay to $t$ instead.

If $m_{t}>m_{b}$, then (in this model) $t$ quarks can decay into $b$ quarks which decay into $c$ quarks. In neutrino scattering $t$ quarks can be produced and decay into two, three or more leptons. For such "trilepton" events the rate would be less than $8 \times 10^{-4} \mathrm{~F}$ of the total rate (counting both $\mu^{-} \mu^{-} \mu^{+}$and $\mu^{-} \mu^{+} \mu^{+}$events).

Trimuon events have been reported by three groups (131). - At Fermilab, one group (131) reports a rate of $10^{-4}$, while at CERN a rate of $5 \times 10^{-5}$ has been reported (131) (in both cases $E_{\nu}>100 \mathrm{GeV}$ and $E_{\mu}>4 \mathrm{GeV}$ is required). Presumably some (and possibly a11) of these events come from background sources (such as $p$ : decay). The rates given above for the WS-GIM model are upper bounds (since upper bounds on $t \bar{d}$ and $u \bar{b}$ couplings are used) and even then appear to be lower than these reported rates, but the question of backgrounds should be resolved. Various studies (132-135) have been made concerning the expected characteristics of such trilepton events; more data are needed before conclusions can be reached.

The multi-lepton events found in $b$ or $t$ quark decays are expected (in the WS-GIM tmodel) to be accompanied by the presence of strange particles. If $m_{b}<m_{t}$, then $\mathrm{b}$ quarks decay into $\mathrm{c}$ quarks which decay into strange particles; and the $t$ quarks would decay into b quarks. If $\mathrm{m}_{\mathrm{t}}<\mathrm{m}_{\mathrm{b}}$, then $t$ quarks would decay dominantly into $s$ quarks directyy; and the b quarks would decay into $t$ quarks. 
While the above remarks were taken in the context of the WS-GIM model, similar conclusions follow in many other models. There are a large variety of left- and right-handed couplings possibie for $b$ and $t$ quarks in both $S U(2) \times U(1)$ and other models, and frequently these result in detectable signals in neutrino experiments as described above.

\section{B. HADROPRODUCTION OF HEAVY PARTICLES}

Heavy vector mesons such as $\psi$ and $\Upsilon$, which decay to $\mu^{+} \mu^{-}$or $e^{+} e^{-}$, have been observed in pp, $\mathrm{pN}, \mathrm{pp}$ and $\pi \mathrm{p}$ scattering experiments (153). There are several different approaches to calculating the cross-sections which have been advocated. Some of them have been modified as further data were reported, and here most attention will be given to the later versions.

One obvious way to produce $\psi$ mesons is by the fusion of a c quark from one of the incoming hadrons with a $\bar{c}$ quark from the other hadron, where the $c$ and $\bar{c}$ quarks come from the "sea" of their respective hadrons (154). The magnitude of such a process is difficult to estimate since assumptions are needed about the validity of $\mathrm{SU}_{4}$ and/or about the manner of decay of the $\psi$ (used to estimate the fusion coupling). However, this process has an unavoidable consequence which is easy to test: all $\psi$ production should be accompanied by the simultaneous production of two charmed particles. The experiments which have been done report (155) that there is no evidence for this process, and it must be quite suppressed compared to other processes.

One modification of such an approach is to argue that the $\psi$ is produced by the fusion of light quarks and antiquarks (154). However, since there are not many antiquarks in a proton compared to an antiproton, one expects $\psi$ production in $\bar{P}$ scattering to be at least twenty times that in pp scattering (depending on the shape of quark and antiquark distributions). Experiment indicates a factor of 
about seven. While this approach appears to be inadequate, such diagrams are included in two other (more successful) methods.

In one of these other approaches it is argied that the P-wave states $(x)$ of the $\psi$ family are produced much more frequently than are $\psi$ 's, and that the observed $\psi^{\prime}$ s are primarily decay products of those P-wave states (156). The production of $\psi^{\prime}$ 's (relative to $X ' s$ ) is said to be suppressed because $\psi$ couplings require at least three gluons, so that the resulting effective coupling is much smaller than that for $x$ which requires only two gluons. In such a picture, the $X$ is produced by two processes: 1) the fusion of two gluons, one from each of the colliding hadrons, and 2) the fusion of a quark and an antiquark from the colliding hadrons. The latter process can be assumed negligible in pp scattering (where there are few antiquarks), but is important in pp scattering. This approach can obtain the correct ratio (157) for $\psi$ production in pp relative to p $\bar{p}$ scattering, which is $0.15 \pm 0.08$ at $\sqrt{\mathrm{s}}=8.75 \mathrm{GeV}$. The cross-section (via gIuons, which are labelled "g" below) can be written as:

$$
\begin{aligned}
\sigma(A+B \rightarrow \psi+X) & =\int d x_{1} d x_{2} f^{A}\left(x_{1}\right) f^{B}\left(x_{2}\right) \sigma(g g \rightarrow x) B(x+\psi+\gamma) \\
& =\frac{8 \pi}{3} r(x \rightarrow g g) B \tau \int_{\tau}^{1} \frac{d x}{x} f^{A}(x) f^{B}\left(\frac{\tau}{x}\right)
\end{aligned}
$$

where $f(x)$ are gluon distribution functions, $\tau \equiv \mathrm{m}^{2} / \mathrm{s}$ and $\mathrm{m}$ is the mass of $x$. Using

$$
\Gamma(x \rightarrow g g) B \approx \Gamma(x \rightarrow \psi+\gamma)=\frac{4 \alpha}{3} e_{Q}^{2} \omega^{3}|<\psi| \vec{r}|x>|^{2} \alpha e_{Q}^{2} m^{-5 / 3}
$$

where $e_{Q}$ is the charge of the quark $\left(\frac{2}{3}\right.$ for charm $)$, $w$ is the $x-\psi$ mass difference, and the last proportionality holds for a linear potential model, then one finds

$$
\sigma(A+B+\psi+X) \approx e_{Q}^{2} m^{-14 / 3} F(\tau)
$$


In this approach both the form and magnitude of $F(\tau)$ can be calculated, and the results shown in Fig. 20 are in reasonable agreement with the data. Even if $F(\tau)$ was biot calculable, one could take $F(\tau)$ from the data for $\psi$ and apply Eq. 6.3 to the production of $T$ and heavier particles.

One motivation for this approach is that it provides an obvious explanation for the observed large suppression of $\psi^{\prime}$ relative to $\psi$ production (153). Since there are no pwave states which can decay into $\psi^{\prime}$, it can only be produced directly; but direct production was assumed to be suppressed. If $\psi$ production is really an indirect process involving $x \rightarrow \psi+\gamma$, then the observation of $\gamma^{\prime} s$ associated with $\psi$ production is a crucial test of this approach (a recent experiment may in fact see such $\left.\gamma^{*} \mathrm{~s}(158)\right)$.

In another approach (159), the production of a pair of quarks, $c$ and $\bar{c}$, is calculated. When the invariant mass of the pair is less than two times the mass of D mesons, it is assumed that $\psi^{\prime}$ 's (or other $\psi$ family members) can be produced. There are three types of diagrams which contribute to $c-\bar{c}$ production in this approach: a) a quark from one of the colliding hadrons can annihilate with an antiquark from the other hadron to give a single gluon which produces a $c-\bar{c}$ pair, b) a gluon from each of the hadrons can couple to a $c$ quark line, producing $a c-\bar{c}$ paix, and $c)$ gluons from each of the hadrons can fuse to a single gluon which produces a $c-\bar{c}$ pair. In each case a color singlet is obtained via final-state interactions which are neglected in calculations. For diagram (a) the cross-section is given by

$$
\sigma=\int_{\left(2 m_{c}\right)^{2}}^{\left(2 m_{\mathrm{l}}\right)^{2}} \frac{\mathrm{d} s^{\prime}}{\mathrm{s}^{3}} \sigma(\mathrm{q} \overline{\mathrm{q}} \rightarrow \mathrm{g} \rightarrow \mathrm{c} \overline{\mathrm{c}}) \mathrm{\tau} \int_{\mathrm{T}}^{1} \frac{\mathrm{dx}}{\mathrm{x}} \mathrm{f}(\mathrm{x}) \mathrm{f}\left(\frac{\mathrm{T}}{\mathrm{x}}\right)
$$

where $s^{\prime}$ is the subenergy, $t=s^{*} / s$ and $f(x)$ are quark distributions. One way to 
estimate $\sigma$ in the integrand is to assume an analogy to Drell-Yan calculations (160) of $\mu^{+} \mu^{-}$production. Then 0 is given by

$$
\sigma \approx \int_{\left(2 m_{c}\right)^{2}}^{\left(2 m_{D}\right)^{2}} d s^{\prime} \frac{d \sigma^{\prime}}{d s^{\prime}} \frac{\alpha^{2}}{\alpha^{2}} \frac{2}{3}
$$

where $a_{s}$ is the strong coupling constant and $\sigma_{D Y}^{\prime}$ is the Drell-Yan cross-section calculated without the quark charges. Diagrams (b) and (c) can be calculated similar1y. When all three types of diagrams are included, one obtains the correct ratio for $\psi$ production in pp relative to p $\bar{p}$ scattering.

In Fig. 20 the data for the production of $\psi$ in proton-nucleon scattering is shown. The curve is a theoretical calculation of Carlson and Suaya using the indirect production $(X \rightarrow \psi)$ approach (156). Other authors using this and other approaches have obtained similar results for $\psi$ production. To test the basic hypotheses of the approaches discussed, one can examine whether they can account for the observed cross-section for $\Upsilon(9.4)$ production. For the indirect production approach one sees from Eq. 6.3 that if the data are adjusted for $e_{Q}^{2} \mathrm{~m}^{-14 / 3}$ and the branching ratio $B\left(\Upsilon \rightarrow \mu^{+} \mu^{-}\right)$, then the $\Upsilon$ data should 1 ie on the same curve as the $\psi$ data. In Fig. 20 these adjusted data are shown with the symbol $\Upsilon$ and do, in fact, lie on the same curve. It was assumed that the quark associated with $\Upsilon$ has charge $-\frac{1}{3}$; otherwise the adjusted data points would 1 ie a factor of about 16 lower (both $e_{Q}^{2}$ and $B$ change by about 4). For the direct production approach, adjusted data points lie below the $\psi$ data by a factor (according to one calculation) of about 5 for charge $-\frac{1}{3}$ quarks and 20 for $\frac{2}{3}$ charge quarks (only $B$ is different for different charges). Since it is probably unreasonable to expect these models for $\Upsilon$ production to be accurate to better than an order of magnitude, these results do not distinguish the two approaches nor are they completely reliable determinations of the charge of the quark in $T$. 
It is interesting to ask what are the bighest mass vector mesons (QD) that can be produced in hadronic collisions at existing and future accelerators and storage rings. In Table 13 (due to Carlson (161)) it is assumed that at least 10 events per year must be observed. Of course, these results are only crude estimates, since extrapolation from $3 \mathrm{GeV}$ particles $(\psi)$ to particles of enormous mass is difficult. There could be unforesen complications; one such complication suggested by Bjorken and by $\mathrm{Nieh}(162)$ is that very massive quarks could have very significant weak decay modes so that the branching ratio to $\mu^{+} \mu^{-}$(or $\mathrm{e}^{+} \mathrm{e}^{-}$) would decrease. The weak decay width becomes a sizable fraction of the total when the quark mass becomes comparable to the $W$ boson mass.

The production of charmed particles $\left(\mathrm{D}^{+} \equiv \mathrm{c} \overrightarrow{\mathrm{d}}, \mathrm{D}^{\circ} \equiv \mathrm{c} \overline{\mathrm{u}}\right.$, etc.) in hadronic collisions has not yet been observed. However, various theoretical estimates suggest that the actual cross-sections are not far below the present experimental limits. There are some simple methods which can be used to estimate cross-sections at Fermilab energies. For example, one could guess .

$$
\frac{\sigma_{\mathrm{D}}}{\sigma_{\psi}} \approx \frac{\sigma_{\mathrm{K}}}{\sigma_{\phi}} \approx 10
$$

This gives $\sigma_{D} \approx 1 \mu \mathrm{b}$ for $\mathrm{pp}$ scattering. Sivers (163) has suggested that one could make use of an assumption that the appropriate transverse momentum scaling variable is $\left(\mathrm{p}_{\perp}^{2}+\mathrm{m}^{2}\right)^{1 / 2}$ rather than $\mathrm{p}_{\perp}$; then the cross-section for $\mathrm{D}$ meson production, might be related to that for pions with $p_{1} \approx 2 \mathrm{GeV}$ so that

$$
\sigma_{D} \approx 0.1 \mathrm{\ell n}\left(\frac{\mathrm{s}}{4 \mathrm{~m}_{\mathrm{D}}^{2}}\right) \exp \left(-\frac{26 \mathrm{~m}}{\sqrt{\mathrm{s}}}\right) \mathrm{mb}
$$

This method requires an assumption about the charmed quark content of nucleons; for the above estimate $\mathrm{c} / \mathrm{s}=0.2$ (where $\mathrm{s}=$ strange quark content) was assumed, which gives $\sigma_{D} \approx 10 \mathrm{mb}$. But there is reason to believe c/s is perkaps an order 
Table 13

The highest mass of $Q \bar{Q}$ mesons which could be produced in pp collisions at existing and proposed facilities (161). The mass calculations include corrections for the existence of weak decay modes (see text); the parenthetical numbers are without such corrections.

\section{Facility}

$\operatorname{ISR}(31+31)$

TRISTAN $(180+180)$

ISABELLE $(400+400)$

FNAL $(270 \div 1000)$

POPAE $(1000+1000)$

UNK $(2000+2000)$

$\operatorname{VBC}\left(10^{4}+10^{4}\right)$

VBA (fixed target)

$$
\sqrt{s}
$$

62

Luminosity

$10^{31}$

360

800

1040

2000

4000

$2 \times 10^{4}$

140

$\frac{\text { Mass (in GeV) for }}{-\frac{1}{3} \text { charge } \frac{2}{3} \text { charge }}$

$21 \quad(21) \quad 26 \quad(26)$

$76 \quad(86) \quad 92 \quad(115)$

$87 \quad(114) \quad 108 \quad(165)$

$90 \quad$ (122) $112 \quad(181)$

94 (140) $117(218)$

96 (151) 121 (243)

98 (161) $124 \quad(276)$

$89 \quad(96) \quad 96(103)$ 
of magnitude smaller, so that $\sigma_{D}$ should be much smaller. The present experimental limit is $\sigma_{\mathrm{D}}<1.5 \mathrm{~kb}$ at $\sqrt{\mathrm{s}}=27 \mathrm{GeV}(164)$.

Hore sophisticated calculations have been carried out $(151,154,156,159,165)$. These are usually extensions of the "direct production" approach to $\psi$ production where the limits of integration over $s^{\prime}$ (such as in Eq. 6.4) are changed to $4 \mathrm{~m}_{\mathrm{D}}^{2}$ and $\mathrm{s}$. As for $\psi$ production, the diagrams $\mathrm{a}, \mathrm{b}$ and $\mathrm{c}$ can all contribute. Babcock, Sivers and Wolfram (165) (among others) discuss the results of such QCD calculations and conclude that diagrams $b$ and $c$ are more important than $a$. They also discuss higher order effects and argue that it is reasonable to neglect them for most purposes. With standard assumptions Babcock et a1. estimate for pp scattering $\sigma_{\mathrm{D}} \approx 1 \mu \mathrm{b}$ at $\sqrt{\mathrm{s}}=27 \mathrm{GeV}$ (also $\sigma_{\mathrm{D}} \approx 10 \mu \mathrm{b}$ at $\sqrt{\mathrm{s}}=54 \mathrm{GeV}$ and $100 \mu \mathrm{b}$ for $\sqrt{\mathrm{s}} \geq 200 \mathrm{GeV}$ ). They argue that the present experimental limit on D production in hadronic collisions favors either smaller values of $\alpha_{s}$ (than expected from lepto-production experiments) or gluon distributions which are more peaked toward small $\mathrm{x}$.

For heavier mesons such as $\bar{Q} \bar{u}$ and $Q \bar{d}$ where $Q \bar{Q} \equiv \cong(9.4)$, most estimates (1.65) are that for a very large range of energies, the cross-sections for production of $\overline{Q d}$ or $Q \bar{u}$ mesons will be two orders of magnitude lower than those for $D$ mesons. This clearly makes observation of such mesons very difficult in hadronic collisions.

In addition to the above calculations, which are based on behavior expected for $y \approx 0$, there have been calculations of periphera1 production (for $x>0.5$ ) of charmed particles. For example, the cross-section (166) for $\pi^{-} \mathrm{p} \rightarrow \mathrm{D}^{-} \mathrm{C}_{0}^{+}$ ( $C$ is a charmed baryon) has been estimated as $0.5 \mathrm{nb}$ while triple Regge calculations (166) of $\pi p \rightarrow$ DX (for $x>0.5$ ) give $\sigma \approx 60 \mathrm{nb}$. 


\section{PHOTOPRODUCTION OF $\psi$ AND CHARM}

One of the earliest papers discussing $\psi$ (prior to its discovery) was written by Carlson and Freund (167) discussing the photoproduction of a then hypothetical $\widehat{c c}$ vector meson. The photoproduction of $\psi$ is usually assumed to be a dominantly diffractive process which can be understood with a modified vector-dominance model (97). The modification allows for the $\gamma-\psi$ coupling to be different at $q^{2}=0$ and $q^{2}=n_{\psi}^{2}$. With this assumption the cross-section (see Ref. 168) is

$$
\frac{d \sigma}{d t}(\gamma N \rightarrow \psi N)=\frac{3 \lambda^{2}}{\alpha M} \Gamma\left(\psi \rightarrow e^{+} e^{-}\right) \frac{d \sigma}{d t}(\psi N \rightarrow \psi N)
$$

where $\lambda$ measures the variation of the $\gamma-\psi$ coupling $g_{\gamma \psi}$ with $q^{2}$ and the off-massshell extrapolation of the invariant amplitude ( $\lambda=1$ for the "naive" vector-dominance mode1). Making use of the optical theorem, one finds:

$$
\left.\frac{d \sigma}{d t}(\gamma N \rightarrow \psi N)\right|_{t_{\min }}=\frac{1}{e^{-b t_{\min }}} \frac{3 \Gamma\left(\psi \rightarrow \mathrm{e}^{+} \mathrm{e}^{-}\right)}{16 \pi \mathrm{M}_{\psi}} \lambda^{2}\left(1+\rho^{2}\right) \sigma_{\operatorname{tot}}^{2}(\psi N)
$$

where do/dt was assumed to have $t$ dependence $e^{-b t}$ (which is consistent with data (i69) for $\left.b=2.9 \mathrm{GeV}^{-2}\right)$ and $\rho \equiv\left(\operatorname{Re} A / I_{m} A\right) \rightarrow 0$ as $s \rightarrow \infty$, with $\mathscr{A}$ the amplitude for $\psi N \rightarrow \psi N$. An independent determination of $\sigma_{\text {tot }}(\psi N)$ can be extracted from the observed A dependence (170) (where $A$ is the effective number of nucleons per nucleus) of $\psi$ photoproduction; experiments on Be and Ta give $\sigma_{\text {tot }}(\psi \mathbb{N})=3.5 \pm 0.8$ ob at $\mathrm{E}_{\gamma}=20 \mathrm{GeV}$. To avoid consideration of threshold factors and of $\rho$, we will issume that this value stays approximately constant up to higher energies $\left(E_{\gamma} \approx 80\right.$ iev). Next, an assumption about the value of $\lambda$ is needed. If the value of the aive vector-dominance model $(\lambda=1)$ is taken, then for $\rho \approx 0$, $\operatorname{do} / \operatorname{dt}(t=0) \approx 400$ $\mathrm{b} / \mathrm{GeV}^{2}$ which is far above the experimental values $(169,171)$ of about $60 \mathrm{nb} / \mathrm{GeV}^{2}$ t $\mathrm{E}_{\gamma} \approx 80 \mathrm{GeV}$. Some theoretical models give $\lambda \approx 0.5$, which gives $\mathrm{d} \sigma / \mathrm{dt}(\mathrm{t}=0) \approx$ $00 \mathrm{nb} / \mathrm{GeV}^{2}$; choosing $\lambda=0.3$ or 0.4 gives 40 or $60 \mathrm{nb} / \mathrm{Gev}^{2}$. Clearly, the naive ector-dominance model must be modified to account for $\psi$ photoproduction. 
From knowledge of $\psi$ photoproduction, there are some immediate implications for the photoproduction of charmed particles. By use of unitarity, it can be shown (168) that for a given energy:

$$
\left.16 \pi \frac{d \sigma}{d t}(\gamma N \rightarrow \psi N)\right|_{t_{\min }} \leq(1+\varepsilon)^{2}\left(1+p^{2}\right)\left(\frac{q^{\psi N}}{q N}\right) \sigma(\gamma N \rightarrow \text { charm }) \sigma(\psi N \rightarrow \text { charm })
$$

where $\varepsilon$ measures violations of the ozI rule and

$$
\frac{q^{\psi N}}{q^{\gamma N}}=\frac{\left[s-\left(m_{p}+m_{\psi}\right)^{2}\right]^{\frac{1}{2}}\left[s-\left(m_{p}-m_{\psi}\right)^{2}\right]^{\frac{1}{2}}}{\left(s-m_{p}^{2}\right)}
$$

(which is $0.33,0.72,0.93$ for $E_{\gamma}=10,20,80 \mathrm{GeV}$ ). Application of the ozI rule again implies that $\sigma_{\text {tot }}(\psi N) \approx \sigma(\psi N \rightarrow$ charm $)$. Using $E_{\gamma}=20 \mathrm{GeV}$ data $(169,171)$ for $(\mathrm{d} \sigma / \mathrm{dt})$ and $\sigma_{\text {tot }}(\psi \mathrm{N})$ in Eq. 6.10, one finds

$$
\sigma(\gamma N+\operatorname{charm}) \geq 115 \mathrm{nb} /(1+\varepsilon)^{2}\left(1+\rho^{2}\right)
$$

Since $\varepsilon$ and $\rho$ are presumably small, it is safe to say $\sigma(\gamma \mathrm{N} \rightarrow \mathrm{charm}) \geq 100 \mathrm{nb}$.

It is possible to estimate the photoproduction of charm by QCD techniques.

A gluon from the nucleon and the incoming photon can each couple to a $c$ quark line producing a $c-\bar{c}$ pair. This has been discussed by various authors $(172,173)$, who find $\sigma(\gamma \mathrm{N} \rightarrow$ charm $)$ increasing from about $100 \mathrm{nb}$ at $\mathrm{E}_{\gamma}=20 \mathrm{GeV}$ to about $400 \mathrm{nb}$ at $\mathrm{E}_{\gamma}=80 \mathrm{GeV}$. Some assumptions are required to apply perturbative OCD to this problem, but the approach is not implausible. Roughly speaking, the large mass of the charmed quark is expected to set the scale for the effective coupling constant. The use of QCD here is similar in some ways to its use in explaining the total width of the $c \bar{c}$ states, and it surely needs further theoretical analysis.

These results are in approximate agreement with a sum rule of Shifman, Vainshtein and Zakharov (173), and may be consistent with the reported observation (118) of 
a charmed antibaryon of mass $2.26 \mathrm{GeV}$. (whose cross-section has not been reported yet). This experiment of knapp et a1. saw evidence for what may have been $\stackrel{\Lambda_{c}}{-} \bar{A} \pi \pi^{-}+$ 


\section{NEUTRAL CURRENT INTERACTIONS}

The weak interactions provide an important probe in the study of new quarks (and new leptons): The neutral-current interactions are a cructal measure not only of the existence of new quarks but also of the structure of the gauge theories of weak and electromagnetic interactions. Were the neutral-current predictions of the WS model to fati, one would be forced to consider other models. In fact, however, the wS model is in good agreement with most neutral-current data, as will be discussed below. The fmportance of neutral-current phenomenology can be seen in the successful prediction of the existence of the $c$ quark from the absence of strangeness-changing neutralcurrents (via the GIM mechanism (4)). For much of the study of neutralcurrent interactions, the neutrino is used as a probe; it is uniquely suited to this purpose, since it is the only particle which has only weak interactions It is possible, a priori, that $c$ quarks (or $b$ or $t$ quarks) could be produced directly by neutral-current processes in which u quarks are changed into $c$ quarks. In the wS model (with all left-handed quarks in doublets, none in singlets) such charm (or other "flavor") changing neutral-currents are forbidden by the GIM mechanism. Two types of experiments indicate that the neutral weak boson $Z_{0}$ (of $S U(2) \times U(1)$ models) does not change charm: 1) $e^{+} e^{-}$ annihilation experiments (174) find no $D^{\circ}-\bar{D}^{\circ}$ mixing (such mixing should be found if charm-changing currents exist); 2) neutrino scattering experiments (17) also find no evidence of $D^{\circ}-\bar{D}^{\circ}$ mixing (which would lead to $\mu^{-} \mu^{-}$events). In a model for a group other than $\mathrm{SU}(2) \times \mathrm{U}(1)$ it might be possible to have flavor-changing neutral-currents if they occur via another boson which is quite heavy or does not have flavor-conserving couplings to 1 ight fermions. For 
$\mathrm{SU}(2) \times \mathrm{U}(1)$ (in which many models are possible) there is little likelihood that there would be $t$ - or b-changing neutral-currents, given that there are no strangeness- or charm-changing currents. In $S U(2) \times U(1)$ models with quarks of charges $\frac{2}{3}$ and $-\frac{1}{3}$ oniy, there are no flavor-changing neutral-currents if all quarks of a given handedness are in doublets (or are all in singlets) of Su(2). If most quarks were in doublets but one were in a singlet, mixing among quarks would lead to flavor-changing neutral-currents for all quarks of that charge (unless there were some reason why mixing was prevented)(115).

There are four types of neutrino experiments which are commonly used to test the diagonal (flavor-conserving) neutral-current structure of gauge theories. These are inclusive scattering off heavy nuclei (175), elastic scattering off protons (176), semi-inclusive (single pion) scattering off heavy nuclei (177), and elastic scattering off electrons (178). The first three can be used to calculate the neutral-current couplings of u and d quarks. In $S U(2) \times U(1)$ models these quark couplings are given by:

$$
\begin{aligned}
& q_{L}=\tau_{3}{ }^{L}-Q \sin ^{2} \theta_{W} \\
& q_{R}=\tau_{3}{ }^{R}-Q \sin ^{2} \theta_{W}
\end{aligned}
$$

where L (R) refers to left-handed (right-handed), $\tau_{3}$ is the weak isospin ( $\pm \frac{1}{2}$ for quarks in doublets, 0 in singlets), $Q$ is the charge of the quark, and $\theta_{W}$ is the "Weinberg" angle, which is a free parameter of this theory. The inclusive and elastic scattering results are usually reported as ratios of neutra1current to charged-current cross-sections for both neutrinos and antineutrinos; the semi-inclusive scattering experiments give ratios of $\pi^{+}$to $\pi^{-}$(in the 
current fragmentation region). With these six numbers, the possible couplings $\left(u_{L}, u_{R}, d_{L}, d_{R}\right)$ are severely limited. Some of the data are shown in Figs. 21 and 22. Analyses have been dore by many authors (179). An analysis of Hung and Sakurai (179) (who make use of conclusions of Sehgal (179)) finds that there are only two sets of couplings for u and d quarks which are allowed by the data. These are (where the uncertainties are a1ways i 0.15):

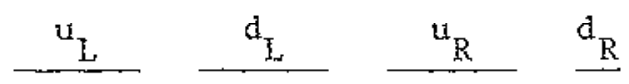

$$
\begin{aligned}
& \mathrm{A} \quad+0.29-0.40-0.24 \quad 0 \\
& \mathrm{~B} \quad+0.29-0.40+0.24 \quad 0
\end{aligned}
$$

Note that if all four signs are changed in set A or in set $B$, the resulting sets of couplings are, of course, equally allowed. If $\sin ^{2} \theta_{\mathrm{W}}=0.3$ is chosen, then the WS model predicts that $u_{L}, d_{L}, u_{R}, d_{R}$ are $0.3,-0.4,-0.2,0.1$. This is very close to set A of allowed couplings. There may be other models such as the $\mathrm{SU}(2) \times \mathrm{U}(1)$ model with ( $\mathrm{u}$ b) ${ }_{\mathrm{R}}$ (see Sec. V) which have values similar to those of set $B$. Note, however, that the parameter $\theta_{W}$ is attributable only to a specific model, and other models may fit the data for different values of that parameter. If there is any shortcoming to the above analysis, it is that these results depended crucially on use of specific parton model assumptions in the analysis of the semi-inclusive data. Since that data was taken at very low energies where parton model assumptions could be questioned, it would be best to confirm the conclusions by independent means, A new analysis, which is near completion, by Abbott and Barnett (179) (based on very new data) will try to use independent methods to further isolate the allowed values of the neutral-current couplings of $u$ and $d$ quarks. 
There are three types of neutrino-electron elastic scattering experiments (178) which have been reported: they are with $\nu_{\mu}, \bar{\nu}_{\mu}$ and $\bar{\nu}_{e}$ beams. With each cross-section one can determine a locus of points in the $g_{A}-g_{V}$ plane which are consistent with the $90 \%$ confidence level upper and lower bounds for that cross-section. Each of these is an annulus and in Fig. 23 the intersection of these three regions (which is shaded) is the allowed region. The WS model with $\sin ^{2} \theta_{W}=0.25-0.3$ lies within the lower part of the allowed region. Some other models lie in the upper part of the allowed region.

There are experiments testing weak neutral-currents which do not involve neutrinos. These concern effects which arise from parfty violation which is possible in weak neutral-currents in contrast to electromagnetic currents (which are purely vector and conserve parity). Among such experiments are those parity-violating transitions in heavy atoms (bismuth, thalium, cesium), in light atoms (hydrogen and deuterium) and in nuclei. There are also experiments which measure polarization asymetries in electron-nucleon deepinelastic scattering and in $\mathrm{e}^{+} \mathrm{e}^{-} \rightarrow \mu^{+} \mu^{-}$.

The experiments involving bismuth are already reporting results which are consistent with zero parity violation. The oxford group finds the measured optical rotation to be $(2.7 \pm 4.7) \times 10^{-8}$ radians, while the washington group finds $(-0.7 \pm 3.2) \times 10^{-8}$ radians $(180)$. The optical rotation measured by this type of experiment on heavy nuclei should be dominated by the interference term $A_{\text {electron }} \mathrm{V}_{\text {hadron }}$ rather than by $\mathrm{V}_{\text {electron }} \mathrm{A}_{\text {hadron. There is some }}$ controversy concerning the atomic and nuclear theory calculations (181, 182); however, the best estimates are that for the WS model the oxford experiment should find $\left(-15 \times 10^{-8}\right)$ radians and the washingt on experiment $\left(-12 \times 10^{-8}\right)$ 
- radians. Within $S U(2) \times y(1)$ one can obtain a zero result for the bismuth experiment if the electron is given vector couplings $\left(A_{e l e c}=0\right.$ ); the electron is vector if it has a coupling $\left(\mathrm{E}^{\circ} \mathrm{e}^{-}\right)_{\mathrm{R}}$ in addition to $\left(v_{\mathrm{e}} \mathrm{e}^{-}\right)_{\mathrm{L}}$ (where $\mathrm{E}^{\circ}$ is a heavy neutral lepton).

There are some models, notably SU(2) ${ }^{\mathrm{L}} \times \mathrm{SU}_{(2)^{\mathrm{R}}} \times \mathrm{U}(1)$ models (see Sec. V), which expect both A electron $\mathrm{V}_{\text {hadron }}$ and $\mathrm{V}_{\text {electron }} \mathrm{A}_{\text {hadron }}$ to be zero (to first order). These models have two weak bosons $\left(z_{\mathrm{V}}^{\mathrm{O}}\right.$ and $\left.2_{\mathrm{A}}^{\circ}\right)$, one which has purely axial-vector couplings to all fermions (except neutrinos) and one which has purely vector couplings. There are experiments which are sensitive to both of the VA terms and have the added feature that they lack the theoretical difficulties of experiments on heavy nuclei (182). These experiments are performed on hydrogen and deuterium, and involve either atomic transitions or electronnucleon deep-inelastic scattering. While the theory is clear, it will be difficult for these experiments to obtain sufficient sensitivity to distinguish among various models. Were the absence of parity violation to be confirmed by these experiments (which expect results in the next year or two), it would be a serious problem for the WS-GIM model.

The neutral-current interactions present a serious challenge to any gauge theory of quarks and leptons. A theory which could account for the wide range of data discussed here would be most impressive. 


\section{CONSERVATION LAWS}

In the construction of gauge theories which incorporate more than four quarks (and leptons), the question of mixing among fermions must be considered again. It was already clear from Cabibbo mixing that the weak interaction eigenstates are not identical to the mass eigenstates. While a deep understanding of the cause of this mixing is still lacking, the phenomenological consequences of it should not be overlooked. One important consequence can be the breakdown of certain conservation laws. Two relevant conservation laws are those for CP (the product of charge-conjugation and parity) and for muon-number. The violation of these quantities is quite small: CP-violating decays of $\mathrm{K}^{\circ}$ mesons are about $10^{-3}$ of $\mathrm{CP}$-conserving decays, and muonmumber violating decays of muons have never been observed and are less than $10^{-8}$ of muon-number conserving decays. The understanding of such conservation laws and their breakdown is a crucial step in building a theory of quarks and leptons.

\section{A. CP VIOLATION}

The theory of $\mathrm{CP}$ violation has been studied (183) for many years. A variety of approaches has been considered involving left-handed and sometimes righthanded currents. Here attention will be linited to the case of the WS model, although some results are applicable to other models. Consideration of CP violation in weak interactions involves not only the question of how it occurs, but also of why it is "milifweak" (CP-violating terms are about $10^{-3}$ of $\mathrm{CP}$-conserving terms). In the WS model, the possibility of CP violation depends in an important way on the number of quarks. If there were only four quarks $(u, c, d, s)$ and only left-handed currents, then $C P$ would be completely conserved in the quark sector. Weinberg (184) and Sikivie (185) have proposed 
that CP violation could occur only in Higgs exchange in such models, which can - automatically give a "milliweak" violation.

If there are six (or more) quarks, then one expects to find CP violation, which a priori need not be small. In contrast to the four-quark case where the weak coupling matrix has one parameter (the Cabibbo angle $\theta_{c}$ ), the wS model with six quarks (discussed first by Kobayashi and Maskawa (137)) has four parameters. They can be taken to be four angles, $\theta_{c}, \theta_{1}, \theta_{2}$ and $\delta$, in terms of which the weak coupling matrix is:

$$
\left(\begin{array}{ccc}
c_{c} & -s_{c} c_{2} & -s_{c} s_{2} \\
s_{c} c_{1} & c_{c} c_{1} c_{2}-s_{1} s_{2} e^{i \delta} & c_{c} c_{1} s_{2}+s_{1} c_{2} e^{i \delta} \\
s_{c} s_{1} & c_{c} s_{1} c_{2}+c_{1} s_{2} e^{i \delta} & c_{c} s_{1} s_{2}-c_{1} c_{2} e^{i \delta}
\end{array}\right)
$$

where the rows correspond to the quarks $u, c$, and $t$, the columns to $d, s$, and $b$, and $C_{c} \equiv \cos \theta_{c}, C_{1} \equiv \cos \theta_{1}$, etc. $C P$ violation cannot be calculated since three angles are not known; however, there are experimental results which limit the possible values of $\theta_{1}$ and $\theta_{2}$ and allow some comment on the expected magnitude of CP violation.

In this generalized case, $\theta_{c}$ must still have the usual value $\left(\theta_{c} \approx 1.3^{\circ}\right)$. From the universality of quark and lepton couplings, El1is et a1. (151) find that the $u \bar{b}$ coupling, $\sin ^{2} \theta_{c} \sin ^{2} \theta_{2}<0.003$ so that $\sin ^{2} \theta_{2}<0.06$. Given this limit, the fact that charmed particles decay dominantly to strange particles leads to no useful limit on $\theta_{1}$ (onIy $\sin ^{2} \theta_{1} \lesssim 0.8$ ). Following the method of Gaillard and Lee (13), the $\mathrm{K}_{\mathrm{I}}-\mathrm{K}_{\mathrm{S}}$ mass difference can set some bounds on $\sin ^{2} \theta_{1}$ (151), depending on several factors: the c quark mass, the $t$ quark mass and the quantitative accuracy of the Gaillard-Lee estimate. If $\cos ^{2} \theta_{2} \approx 1$, then 


$$
\sin ^{2} \theta_{1} \approx\left(a+\sqrt{a^{2}+(f-1) \eta b}\right) / b
$$

where $a \neq \eta+\eta \ln \eta, b \equiv 1+\eta+2 \eta \ell_{n} \eta, \eta \equiv \mathrm{m}_{\mathrm{c}}^{2} / \mathrm{m}_{\mathrm{t}}^{2}$ and $\mathrm{f}$ is a factor measuring the multiplicative deviation from the Gaillard-Lee estimate. Clearly if that estimate were exact $(f=1)$, then $\sin ^{2} \theta_{1}=0$ (note that a is negative). If $\mathrm{f}=2$, one finds $\sin ^{2} \theta_{1}=0.24$ for $n=0.1\left(\mathrm{~m} t=5 \mathrm{GeV}\right.$ ) and $\sin ^{2} \theta_{1}=0.07$ for $\eta=0.01 .\left(m_{t} \approx 15 \mathrm{GeV}\right)$. If $\mathrm{f}=5$, one finds $\sin ^{2} \theta_{1}=0.61 \quad(\eta=0.1)$ and $0.25(\eta=0.01)$

With this information plus a guess for $\delta$, one can (see Refs, 15l, 186) estimate the ratio of the CP-violating to the CP-conserving parts of the $\mathrm{K}^{\circ}$ mass matrix: $|\approx| \approx \frac{1}{\sqrt{2}}\left|\frac{\operatorname{Im} M_{12}^{K}}{\Delta \mathrm{K}}\right| \approx \sqrt{2} \sin \delta \sin \theta_{1} \sin \theta_{2}\left[\frac{b \sin ^{2} \theta_{1}-a}{b \sin ^{4} \theta_{1}-2 a \sin ^{2} \theta_{1}+\eta}\right]$ where $a, b$ and $n$ are defined above and $\delta$ is the phase in the weak coupling matrix, Eq. 8.1. If one chooses $\theta_{c}=\theta_{1}=\theta_{2}=\delta$ (which puts all angles below the experimental upper 1imits), then one Finds the calculated $|\varepsilon|$ to be 10 times the observed value (1.87) (which is about $2 \times 10^{-3}$ ) for $n=0.1$ and 40 times it for $n=0.01$. Alternatively, given the observed CP violation and choosing intermediate values for $\theta_{1}$ and $\theta_{2}$, one can determine $\hat{\theta}_{\text {. For }} \sin ^{2} \theta_{2}$ $=0.03$ and $E=1.5$ (which gives $\sin ^{2} \theta_{1}=0.15$ for $n=0.1$ and 0.05 for $\eta=0.01)$, the values obtained are $\sin ^{2} \delta=2 \times 10^{-t_{4}}$ for $\eta=0.1$ and $\sin ^{2} \delta=$ $5 \times 10^{-5}$ for $n=0.01$.

To summarize, the WS model with six quarks does give CP violation. By choosing the angles in the weak coupling matrix to be sufficiently small, one certainly can obtain the correct magnitude for CP violation. If, however, a random choice of angles is made (within the bounds described above), the predicted CP violation can be one or two orders of magnitude larger than the 
observed violation. While the magnitude of CP violation cannot be predicted accurately, the CP-violating terms are clearly much smaller than those for non-rare decays, so that the usual $\mathrm{K}^{\circ}$. decay phenomenology is obtained qualitatively, It is possible, of course, that there are symmetry arguments or other reasons why $\theta_{1}, \theta_{2}$ and/or $\delta$ must be smal1.

This analysis also gives information concerning the coupling strengths for various charged-current terms which are useful in other sections of this review. Using the coupling matrix 8.1 , the $u \bar{b}$ coupling squared is proportional to $\sin ^{2} \theta_{\mathrm{c}} \sin ^{2} \theta_{2}$, which is less than 0.003 compared to the ud coupling. The t $\bar{d}$ coupling squared is proportional to $\sin ^{2} \theta_{c} \sin ^{2} \theta_{1} \leqslant 0.03$. Furthermore, the ratios of couplings squared for $t \bar{s} / t \bar{d}$ and $c \vec{b} / u \bar{b}$ are both greater than 10 for most but not all angles $\theta_{1}$ and $\theta_{2}$. The small $C P$ violation indicates that at least one of the angles $\theta_{1}, \theta_{2}$ and $\delta$ must be even smaller, but does not indicate which ones.

\section{B. MUON-NUMBER NONCONSERVATION}

Among the interesting tools for understanding the structure of the weak and electromagnetic interactions are experiments searching for processes such as $\mu \rightarrow$ er, $\mu \rightarrow$ eee, and $\mu^{-} \mathrm{N} \rightarrow \mathrm{e}^{-} \mathrm{N}$. While the standard theories expect leptonnumber to be conserved, muon-number may be violated In higher order diagrams. It is assumed that $\mu$ and $v_{\mu}$ have muon-number one and all other particles have zero. Here, three means of finding muon-number violation in SU(2) $\times U(1)$ models will be discussed.

In the context of the WS modei (although it is applicable elsewhere) Bjorken and Weinberg (188) consider the interactions of leptons with Higgs scalars : 


$$
\begin{aligned}
& \mathrm{H}=-\mathrm{g}_{1}\left(\begin{array}{c}
\bar{\nu}_{\mu} \\
-
\end{array}\right)_{\mathrm{L}}\left(\begin{array}{c}
\phi_{1}^{+} \\
\phi_{1}^{\circ}
\end{array}\right) \mu_{\mathrm{R}}^{-}-\mathrm{g}_{2}\left(\begin{array}{c}
v_{\mathrm{e}} \\
\mathrm{e}^{-}
\end{array}\right)\left(\begin{array}{c}
\phi_{2}^{+} \\
\phi_{2}^{\circ}
\end{array}\right) \mu_{\mathrm{R}}
\end{aligned}
$$

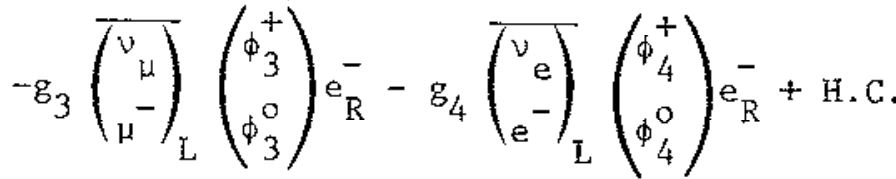

where the $\phi_{i}$ are linear combinations (not necessarily independent) of several scalar fields of definite mass. Since the $\mu$ and e are defined as the physical states found in the diagonalization of the mass matrix, if there is only one Higgs doublet (as is sometimes assumed), then $g_{2}$ and $g_{3}$ must be zero. However, if there is more than one Higgs doublet, then in general it is possible that $g_{2}$ and/or $g_{3}$ are nonzero, and virtual Higgs scalars will give physical transitions between $\mu$ and e such as shown in Fig. 24. Because the Higgs coupling to the light leptons is so weak, the two loop diagrams (Fig. 24b), in general, dominate one loop diagrams (Fig. 24a):

$$
\frac{1 \text { loop }}{2 \text { loops }} \approx \frac{2 \pi}{\alpha}\left(\frac{\mathrm{m}_{\mu}}{\mathrm{m}_{\mathrm{H}}}\right)^{2}
$$

Bjorken and Weinberg roughly estimate

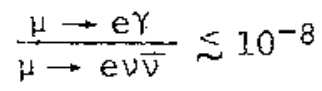

depending on the amount of mixing among the Higgs scalars.

No muon number violation has been observed yet. The present experimental iimit $\left(90 \%\right.$ confidence level) for $\mu \rightarrow$ er is $3.6 \times 10^{-9}$ (189). In the model, the decay $\mu \rightarrow$ 3e was expected to be very sma11. The decay $\mathrm{K}_{\mathrm{L}} \rightarrow$ he is forbidden in lowest order (or one would get strangeness-changing neutra1-currents). They predict 


$$
\frac{\sigma\left(u^{-} N \rightarrow e^{-} N\right)}{\sigma\left(\mu^{-} N \rightarrow v N^{1}\right)} \sim 4 \times 10^{-9}
$$

where $\mathrm{N}$ is a nucleus, and the experimental limit is $1.6 \times 10^{-8}$ (190).

In models which also have right-handed currents there is another source of mun-number violation. This source, discussed first by cheng and li and by Bilenkii et al. (191), involves the mixing of massive neutral leptons which have right-handed couplings to the electron and muon, $\left(\mathscr{A}_{\mathrm{e}}^{\prime} \mathrm{e}^{-}\right)_{\mathrm{R}}$ and $\left(\mathscr{A}_{\mu}^{\prime} \mu^{-}\right)_{\mathrm{R}} \operatorname{In}$ analogy with the Cabibbo mixing of the $d$ and $s$ quarks, they suggest:

$$
\begin{aligned}
\mathscr{N}_{\mathrm{e}}^{\prime} & =\mathscr{N}_{\mathrm{e}} \cos \phi+\mathscr{W}_{\mu} \sin \phi \\
\mathscr{N}_{\mu}^{\prime} & =-\mathscr{N}_{\mathrm{e}} \sin \phi+\mathscr{N}_{\mu} \cos \phi
\end{aligned}
$$

Then clearly if one considers the simple one-loop diagram of Fig. 25, there will be a GIM-1ike cancellation. The cancellation is not complete, to the extent that $\mathscr{N}_{e}$ and $\mathscr{N}_{\mu}$ have unequal masses; the amplitude for this $y \rightarrow$ er process is proportional to

$$
\cos \phi \sin \phi\left(\mathrm{m}^{2}-\pi_{\mu}^{2} \mathscr{N}_{\mathrm{e}}\right)
$$

Bjorken, Lane and Weinberg (192) argue that the Higgs couplings which give masses and lead to the above mixing also cause small but finite mixing of the left-handed parts of $\mathscr{N}_{\mathrm{e}}$ and $\mathscr{N}_{\mu}$ with $v_{\mathrm{e}}$ and $v_{\mu}$. This mixing is of order $\mathrm{m}_{\mu} / \mathrm{m}$. There are, as a result, left-right diagxams in addition to the rightright diagram, Fig. 25. These left-right terms have the same form as the rightright terms, but their amplitude is multiplied by -6 . If the value of expression (8.9) is $1 \mathrm{GeV}^{2}$, then (incorporating the Bjorken-Lane-Weinberg modification)

$$
\frac{\mu \rightarrow \mathrm{e} y}{\mu \rightarrow \mathrm{u} \bar{v}} \approx 4 \times 10^{-10}
$$


Cheng and i estimate the branching ratio for $\mu \rightarrow$ eee to be about $10^{-1}$ (where the experimental limit (193) is $6 \times 10^{-9}$ ), and for $\mu^{-} \mathrm{N} \rightarrow \mathrm{e}^{-} \mathrm{N}$ to be as large as $10^{-9}$. For ${ }_{\mathscr{N}_{\mu}} / \mathrm{m} \mathscr{V}_{\mathrm{e}} \approx 4$, they find the branching ratio for $\mathrm{K}_{\mathrm{L}} \rightarrow \mathrm{e} \bar{\mu}$ to be about $10^{-10}$ (the experimental limit is $2 \times 10^{-9}(194)$ ).

Glashow (195) and Fritzsch (196) have shown that muon number can be violated in models without right-handed currents and with only one Higgs doublet. If the charged heavy lepton $\tau$ has a left-handed coupling to a massive neutral lepton $\mathscr{N}_{\tau}$, then $\mathscr{N}_{\tau}$ can mix with $v_{\mathrm{e}}$ and $\nu_{\mu}$. Decays such as $\mu \rightarrow$ e $\gamma$ could occur in the same fashion as proposed by Cheng and Li and by Bilenkii et al. where $\Delta \mathrm{m}^{2}$ is replaced with ${ }^{2} \mathscr{N}_{\tau}$.

The mixed states can be written as:

$$
\begin{aligned}
v_{\mathrm{e}}^{\prime} & =v_{\mathrm{e}} \cos \theta+\mathscr{N}_{\mathrm{\tau}} \sin \theta \\
v_{\mu}^{\prime} & =v_{\mu} \cos \phi+\left(-v_{\mathrm{e}} \sin \theta+\mathscr{N}_{\mathrm{T}} \cos \theta\right) \sin \phi \\
\mathscr{N}_{\tau}^{\prime} & =\left(\mathscr{N}_{\mathrm{T}} \cos \theta-v_{\mathrm{e}} \sin \theta\right) \cos \phi-v_{\mu} \sin \phi
\end{aligned}
$$

Both angles can be shown to be small by the requirement of universality (seen through $\mu$ - and $\beta$-decay) and by the lack of $\nu_{e}$ in $\nu_{\mu}^{\prime}\left(\nu_{\mu}\right.$ do not produce electrons in scattering). If $\mu \rightarrow$ ey were observed at the $10^{-10}+10^{-9} 1$ evel, then the smallness of the angles $\theta$ and $\phi$ requires that $\mathrm{m} \mathscr{A}_{\tau}$ be quite large (much larger than $m_{\tau}$ in fact). Since $m_{\tau}<m_{\nu}$, the heavy lepton $\tau$ could only decay through the mixing of $\mathscr{N}_{\mathrm{T}}$ with $\nu_{\mathrm{e}}$ and $\nu_{\mu}$.

It would be possible to rule out this mode of muon number violation by measuring the lifetime of $\tau$ carefully, but it probably will be difficuit to obtain a better experimental limit than the present value of about $10^{-11}$ seconds. Since the angle $\phi$ is so small, it would be very rare for $\nu_{\mu}$ scattering to produce t leptons. 
A precise measurement of the conservation or non-conservation of muonzumber would be a valuable tool for studying the existence, mixing and currents of new leptons. 


\section{ATTEMPTS AT A GRAND SYNTHESIS}

Although charm and many predictions of gauge theories for neutral and charged currents seem well verified, the experimental evidence in favor of strong and weak gauge theories remains somewhat indirect. From a theoretical point of view, however, the importance of gauge theories cannot be over-emphasized. They present the first possibility for a theoretically consistent description of both the weak and electromagnetic interactions, and the strong interactions.

In fact, if the gauge theory framework applies generally, there is every reason to believe that within it, a grand unification of all three interactions can be attained. It is an important enterprise to begin working on this even though it is in some ways premature. New questions can be raised and a framework provided for studying unsolved problems such as mass generation and the existence of mixing angles like $\theta_{c}$.

We shall not enter into a detailed discussion of the many models proposed for grand unification. However, there are several features and problems which are necessarily common to any specific model and it is possible to discuss the subject in general terms. This will give the reader some appreciation of these general features and will also serve as something of a conclusion for the entire review.

First of all, it is worth repeating and underscoring the two assumptions which form the foundation for the approach to grand unification to be discussed here.

(1) The weak and electromagnetic interactions are described by a spontaneously broken gauge theory based on some Lie group $G_{w}$, perhaps one of the several models we have discussed. The success of the ws model in 
dealing with neutrino neutral-current interactions indicates that it will contain $S U(2) \times U(1)$ as a subgroup. If $G_{w}$ is Iarger than $S U(2) \times U(1)$, then presumably some of the gauge bosons of $G_{w}$ will be considerably heavier than $M_{W}$ and $M_{Z}$. The quarks and leptons are assumed to fill out Iow dimensional representations of $G_{w}$, with the total number being "reasonably" small. If $\mathrm{G}_{\mathrm{W}}$ is simple (or semisimple in the form $G_{w}^{r} \times G_{w}^{\prime}$, with a reflection symetry relating the two factors), then a single coupling constant is involved. If $S U(2) \times U(1)$ is a subgroup, $\theta_{W}$ will then be determined before proceeding on to grand unification.

With the fermion content arranged to eliminate triangle anomalies, this theory will be renormalizable, and therefore exhibit only logarithmic growth with energy. The coupling strength at laboratory energies wiIl be of order $\alpha=\frac{1}{137}$. At higher energies $E$, the effective coupling constant $\alpha(E)$ (see Sec. II) can change logarithmically but the effect is not significant until a $\log E / E_{l a b}$ becomes of order unity.

(2) The underlying theory of strong interactions is QCD (Eq. 2.2), It is renormalizable, and providing that the total number of quark flavors $f$ is less than 16.5 (Eq. 2.15), it is asymptotically free. The running coupling constant $\alpha_{s}\left(-q^{2}\right)$ for $q^{2}>1-2 \mathrm{GeV}^{2}$ takes the form

$$
\alpha_{s}\left(-q^{2}\right)=\frac{1+\frac{11 \alpha_{\mu}}{4 \pi} \ln \left(\frac{-q^{2}}{\mu^{2}}\right)-\frac{\alpha_{i}}{\pi} \sum_{i=1}^{f} \int_{0}^{1} d z z(1-z) l n\left(\frac{m_{i}^{2}-q^{2} z(1-z)}{m_{i}^{2}+\mu^{2} z(1-z)}\right)}{\alpha_{\mu}}
$$

which agrees with Eqs. 2.15 and $2.16 i \bar{i}-q^{2}, \mu^{2} \gg m_{i}^{2}$. If $-q^{2}, \mu^{2} \ll m_{i}^{2}$ for some flavors, then those quarks can be seen to "decouple" (197) in $\alpha_{s}\left(-q^{2}\right)$. Even if $f>16.5$, a temporary asymptotic freedom could sustain 
itself until a $q$ large enough to vacuum polarize the seventeenth flavor is attained. Nevertheless, in the spirit of assumption (I), we shall take $f<16.5$ so that the asymptotic freedom is truly asymptotic.

The essential notion in superunification is that both QCD and the weak and electromagnetic theory must be viewed as low energy theories. Since they are both renormalizable theories with only logarithmic variation in energy, the range of energies over which they can be viewed as independent theories is necessarily very large. Nevertheless, it is possible to imagine that at some extremely large energy, the strong, weak and electromagnetic interactions will be described by a single theory-a gauge theory of course-based on some Lie group G which contains $G_{W} \times S U_{C}(3)$.

If a spontaneous symmetry breakdown takes place at some extremely large mass scale $M$, then it is possible that some subset of the gauge bosons acquires a mass of order $M$ leaving the subgroup $G_{W} \times S_{C}$ (3) unbroken. The $\mathrm{G}_{\mathrm{w}}$ and $\mathrm{SU}_{\mathrm{C}}(3)$ gauge bosons will remain massless at this leve1. At energy scales somewhat below $M$, the exchange of the superheavy bosons of mass M will be suppressed, and it will appear as if QCD and the weak and electromagnetic theory are two separate field theories. It is possible that the spontaneous symmetry breakdown is a multi-step process. If $\mathrm{G}_{\mathrm{w}}$ is larger than $\mathrm{SU}(2) \times \mathrm{U}(1)$, it could break down at some scale $M^{\prime} \ll M$ (but $M^{\prime} \gg M_{W}, M_{Z}$ ). The final step at mass scale $M_{W}, M_{Z}$ would then leave only the $U(1)$ subgroup intact, with a massless photon. 
That is the scenario in rough outline. Its actual implementation depends on finding the right group $G$ and probably on understanding spontaneous symmetry breaking and the Higgs mechanism much more deeply than we do now. Nevertheless, several features and consequences of the program seem to be understood. We offer a list of those we consider to be most important and then conclude with a partial and subjective list of the many unsolved problems.

(1) If the grand unification group $G$ is simple (or semisimple in the form $G^{\prime} \times G^{\prime}$, with a reflection symmetry relating the two factors), the unified theory will involve a single coupling constant. It is then posm sible to estimate the order of magnitude of $M$, the unification mass scale $(198,199)$. The QCD effective coupling constant $\alpha_{s}\left(-q^{2}\right)$ is already small at $-\mathrm{q}^{2}=10 \mathrm{GeV}^{2}$. Estimates range from about 0.2 based on charmonium decay to about 0.5 based on analyses of electroproduction. This, however, is still much larger than the weak coupling constant (of order $\alpha=\frac{1}{137}$ ), and if these theories are to coalesce into a single coupling constant theory, then something must bring the coupling strengths together. It is primarily the logarithmic decrease of $a_{s}\left(-q^{2}\right)$ with $-q^{2}$ that does this. This decrease can bring $\alpha_{s}\left(-q^{2}\right)$ down to weak and electromagnetic strength at very high momentum scales, but where the effective weak and electromagnetic coupling strength still has not changed much from laboratory energies. The grand unification mass $M$ can be estimated roughly by equating the effective coupling strengths. The result depends on several unknown factors, such as the starting value $\alpha_{\mathrm{S}}\left(10 \mathrm{GeV}^{2}\right)$, the number of quark flavors, the contribution of 
Higgs bosons, and the mass scales characterizing the various possible levels in the symmetry breaking chain. Most estimates (198, 199), however, have ranged between $M \simeq 10^{16} \mathrm{GeV}$ and $M \simeq 10^{19} \mathrm{GeV}$, far beyond laboratory energies. One intriguing feature of these estimates is that they imply the existence of elementary particles with masses on the order of the Planck mass $G^{-1 / 2}=1.22 \times 10^{19} \mathrm{GeV}$. This suggests that grand unification may necessarily involve the gravitational interaction, a possibility to which we return shortly.

(2) The prototype for grand unification into a simple group is the $\mathrm{G}=\mathrm{SU}(5)$ model of Georgi and Glashow (200). It has a maximal subgroup structure of $\mathrm{SU}(2) \times \mathrm{U}(1) \times \mathrm{SU}_{\mathrm{C}}(3)$, so that the usual WS model and QCD are naturally incorporated. If experiments force us to go beyond $\mathrm{SU}(2) \times U(1)$ for $G_{W}$, then some larger group will have to be used for $G$. Some possibilities are $\mathrm{SO}(10)$ (201) and the exceptional groups $\mathrm{E}_{6}$ and $E_{7}$ proposed by Gursey and collaborators (125). $E_{6}$, for example, contains $\mathrm{SU}(3) \times \mathrm{SU}(3) \times \mathrm{SU}_{\mathrm{C}}(3)$ and can accommodate six quarks and four charged leptons in two 27 -plets. The possibility of $G_{w}=S U(3) \times S U(3)$ was already discussed in section $\mathrm{V}$ and it might be an attractive possibility. However, the study of symmetry breakdown in the $\mathrm{E}_{6}$ model indicates that some of the $\mathrm{SU}(3) \times \mathrm{SU}(3)$ bosons will become superheavy so that $\mathrm{G}_{\mathrm{W}}$ is some proper subset of SU(3) $\times$ SU(3) (202).

(3) Once $S U(2) \times U(1)$ is imbedded in a larger simple group, the value of the Weinberg angle is determined. The value of $\sin \theta_{W}$ depends on the structure of $G$ and on the energy scales characterizing the stages of symmetry breakdown to $S U(2) \times U(1)$. In the SU(5) model, for example, the 
value of $\sin ^{2} \theta_{W}$ at the unification mass scale $M$ is $3 / 8$. This will be reduced by renormalization effects at laboratory energies since the effective coupling constants of the $\mathrm{SU}(2)$ and $\mathrm{U}(\mathrm{I})$ subgroups scale differently with q. Numerical estimates lead to $\sin ^{2} \theta_{W} \simeq 0.2(198,199)$ and the range of values allowed by experiment is now $0.2 \leq \sin ^{2} \theta_{W} \leq 0.3$ (see Section VII). A similar prediction is obtained in the $\mathrm{E}_{6}$ model (202). It is premature to take these estimates of $\sin ^{2} \theta_{W}$ too serious $1 y$, but they might be useful in at least excluding some groups $G$. For example, the choice $\mathrm{G}=\mathrm{E}_{7}$ leads to $\sin ^{2} \theta_{\mathrm{W}}=3 / 4$ at the grand unification mass $M$, and it would appear that this is too large to be brought into agreement with experiment by renormalization effects (203).

(4) An important feature of grand unification is that quarks and leptons are placed together in single representations of $G$. Thus, there will be gauge bosons (sometimes called leptoquarks) which connect leptons to quarks and lead to the breakdown of separate lepton and quark conservation. This is a potential disaster since the experimental lower. bound on the lifetime for such transitions is incredibly large. Quark non-conservation can lead to baryon non-conservation, and the lower limit on the proton 1 ifetime is $2 \times 10^{30}$ years (204)! However, if the leptoquark mass is on the order of the grand unification mass M predicted from renormalization group considerations, then lifetimes at least this long long can be obtained. The decay of a proton into a lepton plus pions can proceed by single leptoquark exchange (205), so that the lifetitne will be proportional to $\mathrm{M}^{4} / \mathrm{M}_{\mathrm{H}}^{5}$ where $\mathrm{M}_{\mathrm{H}}$ is some typical hadronic mass scale. Furthermore, the constant of proportionality might be expected to be of order $\alpha^{-2}\left(M^{2}\right) \geq 10^{3}(199)$. With $M_{H} \leq 1 \mathrm{GeV}$ and $M \geq 10^{16} \mathrm{GeV}$, one obtains 
proton lifetimes well in excess of $10^{30}$ years. Although it appears to be sufficiently suppressed, baryon non-conservation is a natural feature of many grand unification models ${ }^{18}$ (205).

The grand unification scenaxio is attractive, but there are too many unanswered questions and loose ends to be sure that it is the wave of the future. A short list of some of these problems should point up the 1imitations of the present theoretical framework, both for dealing with grand unification and perhaps even for understanding the weak and electromagnetic interactions alone.

(1) The nature of spontaneous symmetry breakdown is very poorly understood. The only known way of implementing the breakdown and the Higgs mechanism is by explicitly introducing multiplets of elementary Higgs fields into the Lagrangian. For the $S U(2) \times U(1)$ model, this required only one complex doublet and led to only one physical Higgs boson. However, for the large groups $G$ required for grand unification or even for larger weak and electromagnetic groups $G_{w}$, several very big multiplets of Higgs fields are needed to get a reasonable pattern of symmetry breaking $(201,125)$. The notion of fundamental Higgs fields becomes uneconomical, if not downright unpalatable.

A possibility with some attraction is that the spontaneous symmetry breakdown is "dynamical", that is, not induced by fundamental Higgs fields in the Lagrangian. The Goldstone bosons incorporated into the gauge fields would presumably be bound states of quarks. Whether and how this kind of dynamical symmetry breakdown takes place is unknown, and, in fact, it is hard to see what kind of force could produce the 
necessary binding. Even if dynamical breakdown is a possibility, in moving up from $\mathrm{SU}(2) \times \mathrm{U}(1)$ to $\mathrm{G}$, it is perhaps reasonable to introduce Higgs fields at each stage. They may appeax fundamental at one level if not at a11 energy scales.

(2) There seems to be almost no understanding of quark and lepton masses and weak mixing angles. Hopefully, these fundamental questions will find their solution within the framework of spontaneous symmetry breakdown, but just how is far from clear (206). Many ingredients will presumably enter the solution: the unifying groups $G_{w}$ and $G$, spontaneous symmetry breakdown, and the effects of strong, weak and electromagnetic renormalization. Empirical relations among these parameters, such as $\theta_{\mathrm{c}}^{2} \simeq \mathrm{m}_{\mathrm{d}} / \mathrm{m}_{\mathrm{s}}$ and $\mathrm{m}_{\mathrm{e}} / \mathrm{m}_{\mu} \simeq \mathrm{m}_{\mathrm{u}} / \mathrm{m}_{\mathrm{c}}$, are tantalizing but they could be insidious1y misleading.

(3) Perhaps grand unification into a Lie group G, without also incorporating gravity, is impossible. The natural appearance of mass scales on the order of the Planck mass $\mathrm{G}^{-1 / 2}$ suggests this possibility. At such extremely small distance scales, the gravitational interaction is probably comparable to the other fundamental forces, and it could play an important role, for example, in driving spontaneous symmetry breakdown. Just how this might work is very poorly understood, but there is at least one development which offers promise. The concept of supersymmetry (207), which relates fermions and bosons, has recently been combined with the notion of local gauge invariance to produce a class of theories known as supergravity (208). These theories contain a graviton, one or more spin $3 / 2$ particles known as gravitinos and a host of lower spin particles. 
They have the possibility of being renormalizable (209), but whether or not realistic theories of grand (super-grand?) unification can be constructed along these lines is not yet clear. If supergravity is the road to grand unification, then the group $G$ will not be a simple Lie group. Instead, it will be a graded (or super) Lie group, with an algebra containing both commutation and anticomutation relations.

It is important to think about these deep theoretical questions, but if the recent past is something of a guide, progress will come in more modest steps with experiment playing an important, and possibly leading, role. It is hard to think of another period in particle physics when so many important experiments were either under way or in the planning stages. Within the next few years we may well be able to offer plausible, if not completely accepted, answers to some questions of limited scope but of great import:

(1) Is everything "in order" with charmonium and the charmed particles? The pseudoscalar $\bar{c}$ states are especially puzzling.

(2) To what extent is the upsilon a new, improved charmonium system? Will it give us important information about the quark-antiquark potential in the transition region from linearity to short distances $\left(\lesssim 1 \mathrm{GeV}^{-1}\right) ?$

(3) Will QCD remain a viable theory of strong interactions?

(4) Will spontaneously broken gauge theories remain a viable theoretical framework for weak and electromagnetic interactions? The most crying 
need here is for some evidence in favor of the existence of intermediate vector bosons-perhaps in the mass range $60-100 \mathrm{GeV}$. Do Higgs bosons, either elementary or composite, exist in an accessible mass range?

(5) What is the correct gauge group of weak and electromagnetic interactions $G_{w}$ ? How many quarks and leptons are there and how do they fill out representations of $G_{W}$ ? The weak interaction properties of the $\tau$ lepton and the $b$ quark and the question of parity violation in atomic physics are central here.

(6) What about masses and mixing angles? Are the neutrinos $\nu_{e}, v_{\mu}$ and $\nu_{T}$ massless? Are muon and tau lepton number separately conserved at more stringent levels? Will the pattern of quark and lepton masses offer some clue to the understanding of mass generation?

If the near future provides us with answers to some of these questions, it could be an even more exciting era in particle physics than the near past. 


\author{
ACKNOWLEDGEMENTS \\ We wish to thank many of our colleagues for conversations which \\ were useful in preparation of this review, especially J. Bjorken, C. \\ Carlson, E. Eichten, J. Ellis, G. Feldman, F. Gilman, S. Glashow, K. Gottfried, \\ F. Gursey, F. Martin, C. Quigg, J. Rosner, R. Suaya, and S. Weinberg. Two of \\ us (T.A. and K.L.) wish to acknowledge the hospitality of the SLAC theory \\ group, and two of us (M.B. and K.L.) wish to acknowledge the hospitality of \\ the Yale theory group. It has been a privilege for all three of us to have \\ known and to have learned from Ben Lee.
}




\section{FOOTNOTES}

1. The spinor field $q_{\mathbf{I}}$ is not, strictly speaking, left-handed. The operator $\frac{I}{2}\left(1-\gamma_{5}\right)$ projects out the left-handed (negative helicity) part of the Dirac spinor $u(p)$ only in the zero mass Iimit. Thus for the large momentum $\left(p \gg m_{q}\right)$ components of $q_{L}(x), \frac{1}{2}\left(1-\gamma_{5}\right)$ can be thought of as a covariant version of a left-handed projection operator.

2. This means that the infinities which appear in higher-order computations can all be absorbed into the physical masses and coupling constants of the theory. The S-matrix can then be computed to any order in terms of these few parameters.

3. Although the ${ }^{3} \mathrm{~S}_{1} \overline{c \mathrm{c}}$ ground state was simultaneousiy dubbed $\psi$ (at SLAC) and $J$ (at Brookhaven), the excited states have al1 been discovered at SLAC and DESY in $\mathrm{e}^{+} \mathrm{e}^{-}$annihilation. We shall refer throughout this paper to the $\bar{c}$ states which can be directly produced in $e^{+} e^{-}$annihilation as $\psi, \psi^{\prime}, \psi^{\prime \prime}$, etc.

4. In a renormalizable field theory, the study of scaling properties is not just a simple matter of dimensional analysis. A new dimensional parameter is introduced through renormalization, and that complicates the scaling behavior. The formalism for studying this behavior is called the renormalization group.

5. We use the spectroscopic notation $\mathrm{n}^{2 S+1} \mathrm{~L}_{\mathrm{J}}$, where $\mathrm{n}$ is the number of radial nodes plus one, $S$ is the total $q+\bar{q} \operatorname{spin}(0$ or 1$)$, $L$ is the orbital angular momentum of the $q \bar{q}$ and $J$ is the total angular momentum of the state. 
6. The zero of energy of a system bound in an infinitely rising potential cannot be defined unambiguously.

7. This value of $\alpha_{s}\left(M_{\psi}\right)$ is about a factor of two smaller than that deduced from analyses of electroproduction data.

8. For this, we have used the parameters of Carlson and Gross (Ref. 89).

9. Kogut and Susskind (Ref. 95) implement the coupling to decay channels in a quite different way from Eichten et al. In particular, charmed mesons never appear explicitly.

10. Note that the Hamiltonian in Eq. (3.52) corresponds to the nonrelativistic limit of a pure vector kernel in Eq. (3.33).

11. The experimental justification for the quasi-two-body hypothesis and for the neglect of charmed baryons is presented in Sec. IV.

12. To extract $\Delta R$ from experiment, one should subtract from $R$ a constant background of about 2.2 due to production of non-charmed hadrons plus the contribution of $\tau$ production, which is approximately $\left(1+2 m_{\tau}^{2} / W^{2}\right)\left(1-4 m_{\tau}^{2} / W^{2}\right)^{I / 2}$ at center-of-mass energy $W$.

13. We do not discuss here the possibility that $T$ and $T$ ' are the ground states of two distinct, but nearly degenerate, $\overrightarrow{Q Q}$. See, for example, Cahn and Ellis (103).

14. Quigg and Rosner (105) have recently argued that the number of bound ${ }^{3} \mathrm{~S}_{1}$ levels grows approximately as $2\left(\mathrm{~m}_{\mathrm{Q}} / \mathrm{m}_{\mathrm{c}}\right)^{1 / 2}$.

15. Of course, the fact that only $\mathrm{D}^{\circ}$ and $\mathrm{D}^{+}$(with no $\mathrm{D}^{-}$or $\mathrm{D}^{++}$, say) are observed in the decays $\mathrm{D}^{*+} \rightarrow \mathrm{D}^{+} \pi^{\circ}, \mathrm{D}^{\circ} \pi^{+}$and the fact that the observed $D^{-}$decays are to states of positive strangeness are rather convincing evidence in favor of $I=\frac{1}{2}$ as well as $Q=+2 / 3$ for the charmed quark. 
16. Our notation $\left(q_{I} q_{2}\right)_{L}$ (or $\left.\left(q_{1} q_{2}\right)_{R}\right)$ refers to the charge-changing weak current, Eq. (1.2), with $\left(1-\gamma_{5}\right)$ (or $\left(1+\gamma_{5}\right)$ ) implied. The $w^{ \pm}$boson couples the two fermions, $q_{1}$ and $q_{2}$.

17. The SU(3) used in such models refers to the weak interactions: a triplet might contain $\mathrm{u}, \mathrm{d}$, and $\mathrm{b}$ quarks. It is quite distinct from the old SU(3) associated with the light quarks (u, d and s).

18. It is possible to push the decay to higher orders in the coupling constant. For an extensive discussion of grand unification and proton stability, see Ref. 205. 


\section{LITERATURE CITED}

1. Ge11-Mann, M. 1964. Phys. Lett. 8:214; Zweig, G. 1964. CERN reports TH. 401 and $\mathrm{TH} .412$.

2. Greenberg, 0.W. 1978. To appear in Annual Rev. Nucl. Part. Sci., Vol. 28.

3. Bjorken, J.D., Glashow, S.L. 1964. Phys. Lett. 11:255; Amati, D., et al. 1964. Phys. Lett. 11:190; Tarjanne, P., TepIitz, V.L. 1963. Phys. Rev. Lett. 11:447; Hara, Y. 1964. Phys, Rev. 134:B701; Maki, 2., Ohnuki, Y. 1964. Prog. Theor. Phys. 32:144.

4. Glashow, S.L., I1iopoulos, J., Maiani, L. 1970. Phys. Rev. D2:1285.

5. Carithers, W.C. 1973. Phys, Rev. Lett. 30:1336\& 31:1025;

Fukushima, Y. 1976. Phys. Rev. Lett. 36:348.

6. Cabibbo, N. 1963. Phys. Rev, Lett. 10:531.

7. Weinberg, S. 1967. Phys. Rev. Lett. 19:1264; Salam, A. 1968. Elementary Particle Physics: ReIativistic Groups and Analyticity (Nobel Symposium No. 8). Edited by N. Svartholm. Stockholm: Almquist and Wiksell.

8. Yang, C.N., Mills, R. 1954. Phys, Rev. 96:191.

9. 't Hooft, G. 1971. Nuc1. Phys. B33:173; Lee, B.W. 1972. Phys. Rev. D6:1188; Lee, B.W., Zinn-Justin, J. 1972, Phys. Rev. D5:3132, $3137 \& 3155$.

10. Barish, B.C., et al. 1977. Phys. Rev. Lett. 39:1595.

11. Goldstone, J. 1961. Nuovo Cim. 19:154; Goldstone, J., Salam, A., Weinberg, S. 1962. Phys. Rev. 127:965.

12. Higgs, P.W. 1964. Phys. Rev. Lett. 12:132\&13:508\&1966. Phys. Rev. 145:1156; Guralnik, G.S., Hagen, C.R., Kibble, T.W.B. 1964. 
Phys. Rev. Lett. 13:585; Englert, F., Brout, R, 1964. Phys. Rev. Lett. $13: 321$.

13. Gaillard, M.K., Lee, B.W. 1974. Phys. Rev. D10:897.

14. Litke, A., et al. 1973. Phys. Rev. Lett. 30:1189; Tarnopolsky, G., et a1. 1974. Phys. Rev. Lett. 32:432.

15. Richter, B. 1974. Proc. XVII Int. Conf, on High Energy Physics, London, 1-10 July 1974. Rutherford Laboratory: Science Research Council. p. IV-37.

16. Burmester, J., et a1. 1977. Phys. Lett. 66B:395.

17. Benvenuti, A., et a1. 1975. Phys. Rev. Lett. $35: 1199,1203$, \& 1249; and 1977. Phys. Rev. Lett. 38:1183; Barish, B.c., et al. 1976. Phys. Rev. Lett. 36:939\& 1977. Phys. Rev. Lett. 39:981; von Krogh, J., et al. 1976. Phys. Rev. Lett. 36:710; HoIder, M., et al. 1977. Phys. Lett. 69B:377\& 70B:396; Deden, H., et al. 1977. Phys. Lett. 67B:474; Ba1tay, C., et a1. 1977. Phys. Rev. Lett. 39:62; Bosetti, P., et al. 1977. Phys. Rev. Lett. 38: 1248; Ballagh, H.C., et al. 1977. Phys. Rev. Lett. 39:1650.

18. Augustin, J.-E., et al. 1974. Phys. Rev. Lett. $33: 1406$.

19. Aubert, J.J., et al. 1974. Phys. Rev. Lett. 33:1404.

20. Abrams, G.S., et al. 1974. Phys. Rev. Lett. 33:1453.

21. Braunschweig, W., et al. 1975. Phys. Lett. 57B:407; Feldman, G.J., et a1. 1975. Phys. Rev. Lett. 35:821; Tanenbaum, W.M., et a1. 1975. Phys. Rev. Lett. 35:1323.

22. Goldhaber, G., et a1. 1976. Phys. Rev. Lett. 37:255; Peruzzi, I., et a1. 1976. Phys. Rev. Lett. 37:569. 
23. Herb, S.W., et a1. 1977. Phys. Rev. Lett. $39: 252$; Innes, W.R., et a1. 1977. Phys. Rev. Lett. 39:1240; Kephart, R.D., et al. 1977. Phys. Rev. Lett. $39: 1440$.

24. Perl, M.l., et al. 1975. Phys. Rev. Lett. 35:1489.

25. Per1, M.L., et al. 1977. Phys. Lett. 70B:487.

26. Politzer, H.D. 1973. Phys. Rev. Lett. 26:1346; Gross, D., Wilczek, F. 1973. Phys. Rev. Lett. 26:1343.

27. Greenberg, 0.W. 1964. Phys. Rev. Lett. 13:598.

28. Fritzsch, H. and Ge11-Mann, M. 1973. Proceedings of the XVI Int. Conf. on High Energy Physics, Chicago-Batavia, I11. 1972. Edited by J.D. Jackson and A, Roberts. NAL: Batavia, I11. Vo1. 2, p. 135 and references therein.

29. Bellettini, G., et al. 1970. Nuovo Cim. 66A:243;·Kryshkin, V.I., et a1. 1970. J. Exp. Theor. Phys. 30:1037; Brownan, A., et a1. 1974. Phys, Rev. Lett. 33:1400.

30. Cabibbo, N., Parisi, G., Testa, M. 1970. Nuovo Cim. Lett. 4:35.

31. Rapidis, P., et al. 1977. Phys. Rev. Lett. 39:526.

32. Kirkby, J. 1977. Report SLAC-PUB-2040 (to appear in the proceedings of the 1977 Int. Symp. on Lepton and Photon Interactions at High Energies, Hamburg, Germany, Aug. 25-31, 1977).

33. Yamada, S. 1977. DESY report (to appear in the proceedings of the 1977 Int. Symp, on Lepton and Photon Interactions at High Energies, Hamburg, Germany, Aug. 25-31, 1977).

34. Burmestex, J., et a1. 1977. Phys. Lett. 66B:395; Knies, G. 1977. DESY report $77 / 74$ (to appear in the proceedings of the 1977 Int. Symp. on Lepton and Photon Interactions at High Energies, Hamburg, Germany, Aug. 25-31, 1977). 
35. Abers, E.S., Lee, B.W. 1973. Phys. Rep. 9C:1; for a review of many features of QCD see Marciano, W.J., Pagels, H.R. 1978. Phys. Rep. 36C:137.

36. Fadde'ev, L.D., Popov, V.N. 1967. Phys. Lett. 25B:29.

37. Appelquist, T., Dine, M., Muzinich, I.J. 1977. Phys. Lett. 69B: 231 and Phys. Rev. D to be published.

38. Feinberg, F. 1977. Phys. Rev. Lett. 39:316 and MIT report no. CTP-687; Fischler, w. 1977. Nuc1. Phys. B129:157.

39. Gel1-Mann, M., Low, F. 1954. Phys. Rev. 95:1300; Callan, C. 1970. Phys. Rev. D2:154I; Symanzik, K. 1970. Commun. Math. Phys. 18:227.

40. Bjorken, J.D. 1969. Phys. Rev. 179:1547; in the context of QCD, see 0. Nachtmann. 1977. Heidelberg report HD-THEP-77-13 (to appear in the proceedings of the 1977 Int. Symp. on Lepton and Photon Interactions at High Energies, Hamburg, Germany, Aug. 25-31, 1977).

41. Wilson, K. 1964. Corneli report LNS-64-15 (unpubiished) and 1969. Phys. Rev. 179:1499.

42. Adler, S. 1974. Phys. Rev. D10:3714.

43. Appelquist, T., Georgi, H. 1973. Phys, Rev. D8:4000; Zee, A. 1973. Phys. Rev. D8:4038.

44. Poggio, E., Quinn, H., Weinberg, S. 1976. Phys. Rev. D13:1958; Shankar, R. 1977. Phys. Rev. D15:755.

45. Field, R.D. 1977. Caltech report CALT-68-633.

46. Gaillard, M.K., Lee, B.W. 1974. Phys. Rev. Lett. 33:108; Altare11i, G., Maiani, L. 1974. Phys. Lett. 52B:351; Shifman, M.A., Vainshtein, A.I., Zakharov, V.I. 1977. Nucl. Phys, B120:316.

47. Appelquist, T., Politzer, H.D. 1975. Phys. Rev. Lett. 34:43.

48. Yao, Y.-P. 1976. Phys. Rev. Lett. 36:653; Appelquist, T., et a1. 1976. Phys. Rev. Lett. 36:768; Kinoshita, T., Ukawa, A. 1976. 
Phys. Rev. D13:1573 and 1977. Phys. Rev. D15:1596; Poggio, E., Quinn, H. 1976. Phys. Rev. D14:578:

49. 't Hooft, G. 1977. Deeper Pathways in High Energy Physics--Orbis Scientiae 1977. Edited by A. Perlmuttex and L.F. Scott. Plenum Press: New York, p. 699.

50. Polykov, A. 1975. Phys. Lett. 59B:82; Belavin, A., et al. 1975. Phys, Lett. 59B:85; 't Hooft, G, 1976. Phys. Rev. Lett. $37: 8$.

51. Wilson, K. 1975. Phys. Rev, D10:2445.

52. Kogut, J., Susskind, L. 1975. Phys, Rev. D11:395.

53. De Rújula, A., Glashow, S.L. 1975. Phys. Rev. Lett. 34:46.

54. See, for example, Chang, N.-P., Nelson, C.A. 1975. Phys. Rev. Lett. $35: 1492$.

55. Chew, G.F., Rosenzweig, C. 1976. Nucl. Phys, B104:290.

56. Novikov, V.A., et a1. 1977. Phys. Rev. Lett. 38:626 and 1977 . Phys, Lett. 678:409.

57. Gottfried, K. 1977. Cornel1 report CLNS-376 (to appear in the proceedings of the 1977 Int. Symp. on Lepton and Photon Interactions at High Energies, Hamburg, Germany, Aug. 25-31, 1977). We thank K. Gottfried for use of his figures.

58. Feldman, G.J., Per1, M.L. 1975. Phys. Rep. 19C:234; Schwitters, R.F., Strauch, K. 1976. Ann. Rev. Nucl. Sci. 26:89; Wiik, B.H., WoIf, G. 1977. Report DESY 77/01 (Lectures given at the Les Houches Sunmer School, 1976); Chinowsky, W. 1977. Am. Rev. NucI. Sci. 27:393.

59. Feldman, G.J., Perl, M.L. 1977. Phys. Rep. 33C:285.

60. Okubo, S. 1963. Phys. Lett. 5:165; Zweig, G. 1964. CERN reports TH.401 and TH.412; Iizuka, J. 1966. Suppl. Prog. Theor. Phys. 37-38:21. 
61. Braunschweig, W., et al. 1977. Phys. Lett. 67B:243.

62. Chanowitz, M.S., Gilman, F.J. 1976. Phys. Lett. 63B:178; Lane, K. 1975. Invited talk at the Meeting of the Division of Particles and Fields, American Physical Society, Seattle, Washington, Aug., 1975; Eichten, E., et a1. 1976. Phys. Rev. Lett. 36:500.

63. Eichten, E., et a1, 1975. Phys. Rev. Lett. 34:369.

64. Kang, J.S., Schnitzer, H.J. 1975. Phys. Rev. DI2:841 and 2791; Harrington, B.J., Park, S.Y., Yildiz, A. 1975. Phys, Rev. Lett. $34: 168$ and 706 .

65. Appelquist, T., Politzer, H.D. 1975. Phys. Rev. D12:1404.

66. Tryon, E.P. 1972. Phys. Rev. Lett. 28:1605; Kogut, J., Susskind, L. 1974. Phys. Rev. D9:3501; Wilson, K.G. 1974. Phys. Rev. D10: 2445 .

67. For a review, see Willemsen, J.F. 1974. Proceedings of Summer Institute on Particle Physics, July 29-Aug. 10, 1974. Edited by M.C. Zipt. SLAC, Stanford University. Vol. 1, p. 445.

68. Eichten, E., et al. 1978. Corne11 report CLNS-375 (to appear in Phys. Rev. D). We thank the authors of this paper for use of their results prior to publication.

69. Appelquist, T, et al. 1975. Phys. Rev, Lett. 34:365; Callan, C.G., et al. 1975. Phys, Rev. Lett. 34:52,

70. Martin, A. 1977. Phys. Lett. 67B:330; Grosse, H. 1977. Phys. Lett. 68B:343.

71. Gottfried, K. 1978. Phys. Rev, Lett. 40:598

72. Van Royen, R.P., Weisskopf, V.F. 1967. Nuovo Cim. 50A:617. 
73. Barbieri, R., Gatto, R., Kögerler, R. 1976. Phys. Lett. 60B:183;

Barbieri, R., Gatto, R., Remiddi, E. 1976. Phys, Lett, 61B:465.

74. Chanowitz, M. 1975. Phys. Rev. D12:918; Okun, L., Voloshin, M. 1976. Moscow Report ITEP-95-1976; Brodsky, S.J., et al. 1978. Phys. Lett. 73B: 203.

75. Eichten, E., et a1. 1976. Phys. Rev. Lett. 36:500.

76. Isgur, N, 1976. Phys. Rev. Lett. 36:1262.

77. De Rújula, A., Jaffe, R. 1977. MIT Report CTP-658; Lane, K. 1976. Invited talk at the Meeting of the American Physical Society, Stanford University, Stanford, Calif., Dec. 22-24, 1976.

78. Giles, R.C., Tye, S.-H.H. 1977. Phys. Rev. D16:1079; Horn, D., Mandula, J. 1978. Phys. Rev. D 17:898.

79. Wilczek, F. 1977. Phys, Rev, Lett. 39:1304.

80. Ellis, J., Gaillard, M.K., Nanopoulos, D.V. 1976. Nuc1. Phys. $B 106: 292$.

81. Harari, H. 1976. Phys. Lett. 64B:469.

82. Jackson, J.D. 1977. Report TH.2305-CERN (to appear in the Proceedings of the.XIIth Rencontre de Moriond, Mar. 1977).

83. Duncan, A. 1976. Phys. Rev. D13:2866; Appelquist, T. 1975. Caltech Report CALT-68-499.

84. De Rújula, A., Georgi, H., Glashow, S.L. 1976. Phys. Rev. Dl2: 147.

85. Johnson, K. 1978. Private Communication (MIT Report in preparation).

86. Celmaster, W., Georgi, H., Machacek, M. 1978. Phys. Rev. D17: 879 and 886. 
87. Schnitzer, H.J, 1975. Phys. Rev. Lett. $35: 1540$ and 1975. Phys. Rev. D13:74 and 1976. Phys. Lett. 65B:239.

88. Pumplin, J., Repko, W., Sato, A. 1975. Phys. Rev. Lett. 35:1538.

89. Henriques, A.B., Kellet, B.H., Moorhouse, R.G. 1976. Phys. Lett. 64B:85; Chan, I.-H. 1977. Phys. Lett. 71B:422; Carlson, C.E., Gross, F. 1977. William and Mary Report WM-PP-21 (revised) (to appear in Phys. Rev. D).

90. Rapidis, P.A., et al. 1977. Phys. Rev. Lett. 39:526.

91. Bacino, W., et a1. 1977. Phys. Rev. Lett. 40:671.

92. Chodos, A., et al. 1974. Phys. Rev. D9:3471.

93. Bars, I, 1976. Phys, Rev. Lett. 36:1521; Tye, S.-H.H. 1976. Phys. Rev. D13:3416; Giles, R.C., Tye, S.-H.H. 1977. 'Phys. Rev. Di6:1079; Ng, Y.J., Tye, S.-H.H. 1977. Phys. Rev. D16:2468.

94. Lane, K., Eichten, E. 1976. Phys. Rev. Lett. 37:477.

95. Kogut, J., Susskind, L. 1975. Phys. Rev. Lett. $34: 767$ and 1975. Phys. Rev, D12:2821.

96. Weisskopf, V.F., Wigner, E.P. 1930. Zeit. f. Phys. 63:54 and 65:18; more recent discussions are given in: Gottfried, K. 1970. Brandeis University Sumer Institute in Theoretical Physics 1967. Edited by M. Chrêtien and S. Schweber. Gordon and Breach: New York. Vol. 2.; Dashen, R.F., Healy, J.B., Muzinich, I.J. 1976. Phys. Rev. D14:2773.

97. Sakurai, J.J. 1969. Phys. Rev. Lett. 22:981.

98. Le Yaouanc, A., et al. 1977. Phys. Lett, 71B:397.

99. Goldhaber, G., et a1. 1977. Phys. Lett. 69B:503. 
100. Okun, L.B., Voloshin, M.B. 1976. Zh. Eksp. Theor. Fiz. 23:369 (1976. JETP Lett. 23:333); De Rújula, A., Georgi, H., Glashow, S.L. 1977. Phys. Rev, Lett. 38:317; Bander, M., et al. 1976. Phys. Rev. Lett. 36:695; Rosenzweig, C. 1976. Phys. Rev. Lett. 36:697.

101. Machacek, M., Tomozawa, Y. 1978. Annals Phys. 110:407.

102. Quigg, C., Rosner, J. 1977. Phys. Lett. 71B:153.

103. Cahn, R., Ellis, S.D. 1977. Washington Report RLO-1388-734 (to appear in Phys. Rev. D); Barnett, M. 1977. Report SLAC-PUB-1961 (to appear in the Proceedings of the European Conference on Particle Physics, Budapest, Hungary, 4-9 July 1977); Carlson, C.E., Suaya, R. 1977. Phys. Rev. Lett. 39:908; E11is, J., et al. 1977. Nuc1. Phys. B131:285. Hagiwara, T., Kazama, Y., Takasugi, E. 1978. Phys. Rev, Lett. 40:76.

104. Eichten, E., Gottfried, K. 1977. Phys. Lett. 66B:286.

105. Quigg, C., Rosner, J.L. 1978. Phys. Lett. 72B: 462.

106. Gaillard, M.K., Lee, B.W., Rosner, J.L. I975. Rev. Mod. Phys. $47: 277$.

107. Jackson, J.D. 1976. Proceedings of Summer Institute on Particle Physics, Aug. 2-13, 1976. Edited by M.C. Zipf. SLAC, Stanford University, p. 147.

108. Feldman, G.J. 1977. Report SLAC-PUB-2068 (Lectures at Banff Summer Institute on Particles and Fields, Banff, Alberta, Canada. Aug. 26 $\rightarrow$ Sept. 3, 1977). 
109. Lane,k., Weinberg, S. 1976. Phys. Rev. Lett. $37: 717$; see also Fritzsch, H. 1976. Phys, Lett. 63B:419.

110. Deshpande, N.G.; et a1. 1976. Phys. Rev. Lett. $37: 1305$.

111. Coremans-Bertrand et al. 1976. Phys. Lett. 65B:480; Hand, L., et aI. 3975. Report FNAI-Froposai-382; Burhop, E.H.S., et al. 1976. Phys. Lett. 65B:299; Voyvodic, I. 1976. Report Fermilab-FN-289; Conversi, M. 1975. Report CERN-NP-75-17; Dine, M., et al. 1976. Report Fermilab-Proposa1-P490.

112. Einhorn, M.B., Quigg, C. 1975. Phys. Rev. D12:2015.

113. ElIis, J., Gaillard, M.K., Nanopoulos, D.V. 1975. Nucl. Phys. $B 100: 313$.

114. Altarel1i, G., Cabibbo, N., Maiani, L. 1975. Nuc1. Phys. B88:285 and 1975. Phys. Lett. 57B:277; Kingsley, R.L., et al. 1975. Phys. Rev. D11:1919 and 1975. Phys. Rev. D12:106; Pais, A., Rittenberg, V. 1975. Phys. Rev. Lett. 34:707.

115. Glashow, S.I., Weinberg, S. 1977. Phys. Rev. D15:1958; Paschos, E.A. 1977. Phys. Rev. D15:1966; Gaillard, M.K., Lee, B.W., Rosner, J.L. 1975. Rev. Mod. Phys. 47:277. Kingsley, R.L., et al. 1975. Phys. Rev. D11:1919 and D12:106; Okun, L.B., Zakharov, V.I., Pontecorvo, B.M. 1975. Lett. Nuovo Cim, 13:218; De Rüjula, A., Georgi, H., Glashow, S.L. 1975. Phys. Rev. Lett. 35:69.

116. Brandelik, R., et al. 1977. Phys. Lett. 70B:132.

117. Cazzoli, E.G., et al. 1975. Phys. Rev. Lett. 34:1125.

118. Knapp, B., et aI. 1976. Phys. Rev. Lett. 37:882.

119. Wiss, J., et al. 1976. Phys. Rev, Lett. 37:1531.

120. Brandelik, R., et al. 1977. Phys. Lett. 70B:387, 
121. Feller, J.M., et al. 1978. Phys. Rev. Lett. 40:274.

122. Kirkby, J. 1977. Report SLAC-PUB-2040 (to appear in the Proceedings of the 1977 Int. Symp. on Lepton and Photon Interactions at High Energies, Hamburg, Germany, Aug. 25-31, 1977).

123. Barnett, M. 1975. Phys. Rev. Lett. 34:41, 1975. Phys. Rev. D11:3246 and 1976. Phys. Rev. D13:671; Fayet, P. 1974. Nuc1. Phys. B78:14; Gürsey, F., Sikivie, P. 1976. Phys. Rev. Lett. 36: 775; Ramond, P. 1976. Nucl. Phys. B110:214.

124. See, for example, De Rújula, A., Georgi, H., Glashow, S.L. 1975. Phys. Rev. D12:3589; Wilczek, F.A., et al. 1975. Phys. Rev. D12: 2768; Fritzsch, H., Gell-Mann, M., Minkowski, P. 1975. Phys. Lett. 59B:256; Pakvasa, S., Simmons, W.A., Tuan, S.F. 1975. Phys. Rev. Lett. $35: 702$.

125. Guirsey, F., Sikivie, P. 1976. Phys. Rev. Lett. $36: 775$ and 1977. Phys. Rev. D16:816; Ramond, P. 1976. Nuc1. Phys. B110:214 and 1977. Nucl. Phys. BI26:509; Giirsey, F., Ramond, P., Sikivie, P. 1976. Phys. Lett. 60B: 177 and 1975. Phys. Rev. D12:2166; Guirsey, F., Serdaroglu, M. 1978. Nuovo Cim. Lett. 21: 28.

126. Horn, D., Ross, G.G. 1977. Phys. Lett. 67B:460.

127. Adier, S.L., 1970. Lectures on Elementary Particles and Quantum Field Theory. Edited by S. Deser, M. Grisaru, and H. Pendleton. MIT Press: Cambridge, Mass.; Gross, D.J., Jackiw, R. 1972. Phys. Rev. D6:477.

128. Knies, G. 1977. DESY report $77 / 74$ (to appear in the Proceedings of the 1977 Int. Symp. on Lepton and Photon Interactions at High Energies, Hamburg, Germany, Aug. 25-31, 1977). 
129. Benvenuti, A., et a1. 1976. Phys. Rev. Lett. 36:1478 and 37:189. 130. Barish, B.C., et al. 1977. Phys. Rev. Lett. $39: 741$ and 1595, and 1977. Caltech report CALT-68-626 (to appear in the Proceedings of the 1977 Int. Symp. on Lepton and Photon Interactions at High Energies, Hamburg, Germany, Aug. 25-31, 1977); Holder, M., et al. 1977. Phys. Rev. Lett. 39:433; Steinberger, J. 1977. CERN Report (lectures presented at the Summer Institute, Cargèse, France, 4-23 JuIy 1977); Schultze, K. 1977. Aachen Report PITHA-92 (to appear in the Proceedings of the 1977 Int. Symp. on Lepton and Photon Interactions at High Energies, Hamburg, Germany, Aug. 25-31, 1977); Bosetti, P.C., et a1. 1977. Phys. Lett. 70B:273; Berge, J.P., et a1. 1977. Phys. Rev. Lett. 39:382.

131. Barish, B.C., et a1. 1977. Phys. Rev. Lett. 38:577; Benvenuti, A., et aI. 1977. Phys. Rev. Lett. $38: 1110$ and 1183, and 1978. Phys. Rev. Lett. 40:488; Holder, M., et al. 1977. Phys. Lett. 70B:393.

132. Barger, V., et al. 1977. Phys. Rev. Lett. $38: 1190$ and 1977. Phys. Rev. D16:2141; Albright, C.H., Smith, J., Vermaseren, J.A.M. 1977. Phys. Rev. Lett. 38:1187; and 1977. Phys. Rev. D16:3182 and 3204; and 1977. Stony Brook report ITP-SB-77-63; Albright, C.H., Shrock, R.E., Smith, J. 1977. Report Fermilab-PUB-77/81-THY; Zee, A., Wilczek, F., Treiman, S.B. 1977. Phys. Lett. 68B:369; Barnett, M., Chang, L.N. 1977. Phys. Lett. 72B:233; Barnett, M., Chang, L.N., Weiss, N. 1977. Report SLAC-PUB-2063 (to appear in Phys. Rev. D); Langacker, P., Segre, G. 1977. Phys. Rev. Lett, 39:259; Barger, V., et al. 1977. Phys. Lett. 70B:329 and 1977. Phys. Rev. D16:3170; Cox, P.H., Yildiz, A. 1977. Phys. Rev. D16:2897; Pakvasa, S., Sugawara, H., Suzuki, M. 1977. Phys. Lett. 69B:461. 
133. Barnett, M., Chang, L.N. 1977. Phys. Lett. 72B:233; Barnett, M., Chang, L.N., Weiss, N. 1977. Report SLAC-PUB-2063 (to appear in Phys. Rev. D); Langacker, P., Segre, G. 1977. Phys. Rev. Lett. 39:259; Albright, C.H., Smith, J., Vermaseren, J.A.M. 1977. Stony Brook report ITP-SB-77-63; Albright, C.H., Shrock, R.E., Smith, J. 1977. Report Fermilab-PUB-77/81-THY.

134. Bletzacker, F., Nieh, H.T., Soni, A. 1977. Phys. Rev, Lett. 38:1241; Soni, A. 1977. Phys. Lett. 71B:435; Goldberg, H. 1977. Phys. Rev. Lett. 39:1598; Young, B.-L., Walsh, T.F., Yang, T.C. 1977. Report DESY-77/80; and the papers of Barnett-Chang, BarnettChang-Weiss, and Albright-Smith-Vermaseren in Ref. 133.

135. Smith, J., Vermaseren, J.A.M. 1977. Stony Brook Report ITP-SB77-66; Barnett, M., Chang, L.N., Weiss, N. 1977. 'Report SLAC-PUB2063 (to appear in Phys. Rev. D); Barger, V., GottschaIk, T., Phillips, R.J.N. 1977. Wisconsin Report Co0-881-9.

136. See, for example, Barnett, M. 1977. Deeper Pathways in High Energy Physics--0rbis Scientiae 1977. Edited by A. Perlmutter and L.F. Scott. Plenum Press: New York, p. 389.

137. Kobayashi, M., Maskawa, K. 1973. Prog. Theor. Phys. 49:652; see also: Harari, H. 1975. Phys. Lett. 57B:265, and 1975. Annals Phys. $94: 391$.

138. Golowich, E., Holstein, B.R. 1975. Phys. Rev. Lett. 35:831, and 1977. Phys. Rev. D15:3472; Branco, G., Mohapatra, R.N. 1976. Phys. Rev. Lett. 36:926; Wilczek, F.A., et al. 1975. Phys. Rev. D12:2768; Branco, G., Mohapatra, R.N., Hagiwara, T. 1976. Phys. Rev. D13:680. 
139. Pati, J., Salam, A. 1974. Phys, Rev. D10:275; Fritzsch, H., Minkowski, P. 1976. Nucl. Phys. B103:6I; Mohapatra, R.N., Sidhu, D.P. 1977. Phys. Rev. Lett. 38:667. De Rújula, A., Georgi, H., Glashow, S.L. 1977. Anna1s Phys. 109:258; Bég, M.A.B., Zee, A. 1973. Phys. Rev. Lett. 30:675 and 1973. Phys. Rev. D8: 1460; Bég, M.A.B., et al. 1977. Phys. Rev. Lett. 38: 1254; Bég, M.A.B., et a1. 1977. Phys. Rev. Lett. $39: 1054$.

140. Georgi, H., Weinberg, S. 1978. Phys. Rev. D17:275.

141. Segre, G., Weyers, J. 1976. Phys. Lett. 65B:243; Lee, B.W., Weinberg, S. 1977. Phys. Rev. Lett. 38:1237; Lee, B.W., Shrock, R. 1977. Report Fermilab-PUB-77/48-THY; Barnett, M., Chang, I.N. 1977. Phys. Lett. 72B:233, and SLAC report in preparation; Barnett M., Chang, L.N., Weiss, N. 1977. Report SLAC-PUB-2063 (to appear in Phys. Rev. D); Langacker, P., Segxe, G., 1977, Phys. Rev. Lett. 39:259; Langacker, P., Segre, G., Golshani, M. 1978. Phys. Rev. D17:1402.

142. Bjorken, J.D., Lane, K. 1978. SLAC Report.

143. Pati, J.C., Salam, A. 1975. Phys. Lett. 58B:333, and 1977. Proceedings--Neutrino '76, Aachen, June 1976. Edited by H. Faissner, H. Reithler, P. Zerwas. Friedr. Viewig und Sohn: Braunschweig.

144. Han, M.-Y., Nambu, Y. 1965. Phys, Rev. 139:B1006.

145. Barnett, M. 1977. Proceedings--Particles and Fields '76. (Brookhaven APS meeting 1976.) Edited by H. Gordon and R.F. Peierls. Brookhaven National Laboratory, p. D77.

146. Holder, M., et al. 1977. Phys. Lett. 69B:377.

147. Altarelii, G., Parisi, G., Petronzio, R. 1976. Phys. Lett. 63B:183; Altarelli, G., Parisi, G. 1977. Nucl. Phys. B126:298; Barnett, M., Georgi, H., Politzer, H.D. 1976. Phys. Rev, Lett. 
37:1313; Kaplan, J., Martin, F. 1976. Nucl. Phys. Bl15:333;

Barnett, M., Martin, F. 1977. Phys. Rev. D16:2765; Zakharov, V.I.

1977. Proceedings of the XVIII International Conference on High

Energy Physics, Tbilisi, July 1976. Edited by N.N. Bogoliubov et al. Joint Institute for Nuclear Research: Dubna. Vol. II, p. B69.

Zee, A., Wilczek, F., Treiman, S.B. 1974. Phys. Rev, D10:2881;

Buras, A.J., Gaemers, K.J.F. 1977. Phys. Lett. 71B:106; Buras,

A.J. 1977. Nucl. Phys. B125:125; Buras, A.J., et al. 1977.

Nucl. Phys. B131:308; Fox, G.C. 1977. Caltech Report CALT-68-619;

Baluni, V., Eichten, E. 1976. Phys. Rev, Lett. $37: 1181$.

148. Chang, L.N., Derman, E., Ng, J.N. 1975. Phys. Rev. Lett, $35: 6$ and 1252; and 1975. Phys. Rev. D12:3539; Chang, L.N., Ng, J.N. 1977. Phys. Rev. D16:3157; Pais, A., Treiman, S.B. 1975. Phys. Rev. Lett. 35:1206; Derman, E. 1976. Nucl. Phys. B110:40.

149. Baitay, C., et al. 1977. Phys. Rev. Lett. 39:62.

150. von Krogh, J, et al. 1976. Phys. Rev. Lett. 36:710; Schu1tze, K. 1977. Aachen Report PITHA-92 (see Ref. 130).

151. Ellis, J., et al. 1977. Nucl. Phys. B131:285; El1is, J., Gaillard, M.K., Nanopoulos, D.V. 1976. Nuc1. Phys. B109:213.

152. Cahn, R.N., Ellis, S.D. 1977. Phys. Rev. D16:1484; A1i, A. 1977. Report CERN-TH-2411.

153. Aubert, J.J., et a1. 1975. Nucl. Phys. B89:1; Blanar, G.J., et a1. 1975. Phys. Rev. Lett. 35:346; Knapp, B., et a1. 1975. Phys. Rev. Lett. 34:1044; Büsser, F.W., et al. 1975. Phys. Lett. 56B: 482; Anderson, K.J., et a1, 1976. Phys. Rev. Lett. 36:237; Antipov, Y.M., et al. 1976. Phys. Lett. 60B:309; Snyder, H.D., 
et a1. 1976. Phys. Rev. Lett. 36:1415; Branson, J.G., et a1. 1977. Phys. Rev. Lett. 38:1334; Cordon, M.J., et al, 1977. Phys. Lett. 68B:96; Bushnin, Y.B., et a1. 1977. Phys. Lett. 72B:269; Cobb, J.H., et a1. 1977. Phys. Lett. 68B:101; Ama1di, E., et al. 1977. Lett. Nuovo Cim. 19:152; Bamberger, A., et a1. 1977. CERN report.

154. Sivers, D. 1976. Nucl. Phys. B106:95; Barnett, M., Silverman, D. 1975. Phys. Rev. D12:2037; Gunion, J. 1975. Phys. Rev. D12:1345; Green, M.B., Jacob, M., Landshoff, P. 1975. Nuovo Cim. 29A:123; Donnachie, A., Landshoff, P. 1976. Nuc1, Phys. B112:233.

155. Binkley, M., et al. 1976. Phys. Rev. Lett. 37:578.

156. Ellis, S., Einhorn, M., Quigg, C. 1976. Phys. Rev. Lett. 36:1263; Carlson, C.E., Suaya, R. 1976. Phys, Rev. D14:3115, and 1977. Phys. Rev. D15:1416, and 1978. Report SLAC-PUB-2076; EIlis, S., Einhorn, M. 1975. Phys. Rev. D12:2007; for $T$ production, see: Cahn, R., Ellis, S. 1977. Phys. Rev. D16:1484 and the papers in Ref. 103.

157. Cordon, M.J., et a1. 1977. Phys. Lett. 68B:96.

158. Cobb, J.H., et al. 1978. Phys. Lett. 72B:497.

159. Fritzsch, H. 1977. Phys. Lett. 67B:217; Halzen, F. 1977. Phys. Lett. 69B:105; Gaisser, T.K., Halzen, F., Paschos, E. 1977. Phys. Rev. D15:2577; Gluck, M., Owens, J.F., Reya, E. 1977. FIorida State Report FSU-HER-770810; for T production, see: E11is, J., et a1. 1977. Nucl. Phys. B131:285; Jones, L., Wyld, H. 1977. Illinois Report I11-TH-77-35; Owens, J., Reya, E. 1977. Florida State Report FSU-HEP-770920. 
160. Dre11, S., Yan, T. - M. 1970. Phys. Rev. Lett. 25:316.

161. Carlson, C.E. Private communication.

162. Bjorken, J.D. 1977. Report SLAC-PUB-2041 (to appear in the Proceedings of the 1977 Int. Symp. on Lepton and Photon Interactions at High Energies, Hamburg, Germany, Aug. 25-31, 1977); Nieh, H.T. 1977. Stony Brook Report ITP-SB-77-64.

163. Sivers, D, 1976. Nucl. Phys. B106:96.

164. Coremans-Bertrand, G., et a1. 1976. Phys. Lett. 65B:480.

165. Babcock, J., Sivers, D., Wolfram, S. 1977. Argonne Report ANL-HEPPR-77-68; Gaisser, T.K., Halzen, F., Kajantie, K. 1975. Phys. Rev. D12:1968; Pilachowski, L., Tuan, S.F. 1975. Phys. Rev. D11:3148; Barnett, M. 1975. Phys, Rev. D12:3441; Gaisser, T.K., Halzen, F. 1975. Phys. Rev. D11:3157, and 1976. Phys. Rev. D13:171; Hinchliffe, I., Llewellyn Smith, C.H. 1976. Phys. Lett. 61B:472, and 1976. NucI. Phys. B114:45; Bourquin, M., Gaillard, J.M. 1976. Nuc1. Phys. B114:334; McKay, D.W., Young, B.-L. 1977. Phys. Rev. D15:1282; Cox, P.H., Park, S.Y., Yildiz, A. 1977. Phys. Lett. 70B:317; Jones, L.M., Wyld, H.W. 1977. IlIinois Report I11-TH-7732; Gustafson, G., Peterson, C. 1977. Phys. Lett. 67B:81; Halzen, F., Matsuda, S. 1978. Phys. Rev. D17:1344.

166. Barger, V., Phillips, R. 1975. Phys. Rev. D12:2623; Field, R.D., Quigg, C. 1975. Report Fermilab-75/15-THY.

167. Carlson, C.E., Freund, P.G.O. 1972. Phys. Lett. 398:349.

168. Sivers, D., Townsend, J., West, G. 1976. Phys. Rev. D13:1234; Walsh, T. 1975. Nuovo Cim. Lett. 14:290; Boreskov, K.G., Ioffe, B.L. 1976. Moscow Report ITEP-102-1976; Ioffe, B.L., Okun, L.B., 
Zakharov, V.I. 1975. Moscow-ITEP Report; Aviv, R., et al. 1975. Phys. Rev. D12:2862; Pumplin, J., Repko, W. 1975. Phys. Rev. D12:1376; Horn, D. 1975. Phys, Lett. 58B:323; Humpert, B., Wright, A.C.D. 1977. Phys. Rev. D15:2503; Humpert, B. 1977. Phys. Lett. $68 \mathrm{~B}: 66$.

169. Camerini, U., et al. 1975. Phys. Rev. Lett. 35:483.

170. Anderson, R.L., et al. 1977. Phys. Rev. Lett. 38:263.

171. Gittleman, B., et a1. 1975. Phys. Rev. Lett. 35:1616; Knapp, B., et al. 1975. Phys. Rev. Lett. 34:1040; Nash, T., et al. 1976. Phys. Rev. Lett. 36:1233.

172. Babcock, J., Sivers, D., WoIfran, S. 1977. Argonne Report ANLHEP-PR-77-68; Chen, M.-S., Kane, G.L., Yao, Y.-P. 1976. Michigan Report UM HE 76-17; Novikov, V.A., et a1. 1977. Moscow Report ITEP-123-1977; Jones, L.M., WyId, H.W. 1978. Phys. Rev. D17:759.

173. Shifman, M.A., Vainshtein, A.I., Zakharov, V.I., 1976. Phys. Lett. $65 B: 255$.

174. Feldman, G.J., et al. 1977. Phys. Rev. Lett. $38: 1313$.

175. Blietschau, J., et al. 1977. Nucl. Phys. B118:218; Benvenuti, A., et al. 1976. Phys. Rev. Lett. $37: 1039$; Barish, B.C., et a1. 1977. Caltech Report CALT-68-601; HoIder, M., et al. 1977. Phys. Lett. 71B:222 and 72B;254; Schultze, K. 1977. Aachen Report PITHA-92 (to appear in the Proceedings of the 1977 Int. Symp. on Lepton and Photon Interactions at High Energies, Hamburg, Germany, Aug. $25-31,1977)$. 
176. Cline, D., et aI. 1976. Phys. Rev. Lett. $37: 252$ and 648; Lee, W., et al. 1976. Phys. Rev. Lett. 37:186; Sulak, 1.R., et a1. 1977. Proceedings--Neutrino '76, Aachen, June, 1976. Edited by H. Faissner, H. Reithler, P. Zerwas. Friedr. Viewig und Sohn: Braunschweig; Poh1, M., et al. 1978. Phys. Lett. 72B:489.

177. KIuttig, H., Morfin, J.G., Van Doninck, W. 1977. Phys. Lett. $71 B: 446$.

178. Hasert, F.J., et al. 1973. Phys. Lett. 46B:121; Blietschau, J., et a1. 1976. Nuc1. Phys. B114:189; Reínes, F., et al. 1976. Phys. Rev, Lett. 37:315; Reithler, H. 1977. Aachen Report PITHA1977-95 (to appear in the Proceedings of the 1977 Int. Symp. on Lepton and Photon Interactions at High Energies, Hamburg, Germany, Aug. 25-31, 1977).

179. Sehga1, L.M. 1977. Phys. Lett. 71B:99; Hung, P.Q., Sakurai, J.J. 1977. Phys. Lett. 72B:208; Abbott, L., Barnett, M. 1978. Phys. Rev. Lett. 40:1303; Barnett, M. 1976. Phys. Rev. D14:2990; Albright, C.H., et al. 1976. Phys. Rev. D14:1780; Barger, V., Nanopoulos, D.V. 1977. Nuc1. Phys, B124:426; Sidhu, D.P. 1976. Phys. Rev. D14:2235; Bernabeu, J., Jar1skog, C. 1977. Phys. Lett. 69B:71; Langacker, P., Sidhu, D.P. 1978. Brookhaven Report.

180. Baird, P.E.G., et al. (Oxford and Washington experiments). 1976. Nature 264:528; Sandars, P.G.H. (to appear in the Proceedings of the 1977 Int. Symp. on Lepton and Photon Interactions at High Energies, Hamburg, Germany, Aug. 25-31, 1977).

181. Feinberg, G. 1977. Columbia Report CU-TP-111 (to appear in the Proceedings of the Ben Lee Memorial Int. Conf., Batavia, I1linois, oct. 20-22, 1977). 
182. Cahn, R.N., Kane, G.L. 1977. Phys. Lett. 71B:348; Marciano, W.J., Sanda, A.I. 1978. Rockefeller Report COO-2232B-142.

183. Sachs, R.G. 1963. Ann. Phys. 22:239; Wolfenstein, L. 1964. Phys. Rev. Lett. 13:562; Mohapatra, R.N. 1972. Phys. Rev. D6:2023; Pais, A. 1973. Phys. Rev. D8:625; 1972. Phys. Rev. Lett. 29:1719, and 1973. Phys. Rev. Lett. 30:114; Lee, T.D. 1973. Phys, Rev. D8:1226, and 1974. Phys. Rep. 9C:143; Mohapatra, R.N., Pati, J.C., Wo1fenstein, L. 1975. Phys. Rev. D11:3319.

184. Weinberg, S. 1976. Phys. Rev. Lett. 37:657.

185. Sikivie, P. 1976. Phys. Lett. 65B:141.

186. Maiani, L. 1976. Phys. Lett. 62B:183; Pakvasa, S., Sugawara, H. 1976. Phys. Rev. D14:305.

187. Geweniger, C., et al. 1974. Phys. Lett. 48B:487; Messner, R., et aI. 1973. Phys. Rev. Lett. 30:876.

188. Bjorken, J.D., Weinberg, S. 1977. Phys. Rev. Lett. 38:622.

189. Depommier, P., et a1. 1977. Phys. Rev. Lett. 39:1113.

190. Bryman, D.A., et aI. 1972. Phys. Rev, Lett. 28:1469.

191. Cheng, T.-P., Li, L.-F. 1977. Phys. Rev. Lett. $38: 381$ and 1977. Phys, Rev. D16:1425; Bilenkii, S.M., Petkov, S.T., Pontecorvo, B. 1977. Phys. Lett. 67B:309.

192. Bjorken, J.D., Lane, K., Weinberg, S. 1977. Phys. Rev. D16:1474. This paper refers to much of the Iiterature on muon-number violation.

193. Korenchenko, S.M., et al, 1976. Sov. Phys.-JETP 43:1.

194. Fitch, V.L., et a1. 1967. Phys. Rev. 164:1711.

195. GIashow, S.L. 1977. Harvard report HUTP-77/A008. 
196. Fritzsch, H, 1977. Phys, Lett, 67B:451.

197. Appelquist, T., Carazzone, J. 1975. Phys. Rev. D11:2856.

198. Georgi, H., Quinn, H., Weinberg, S, 1974. Phys. Rev, Lett. $33: 451$.

199. Buras, A.J., et al. 1977. Report CERN-TH-2403.

200. Georgi, H., Glashow, S.I. 1974. Phys. Rev. Lett. 32:438.

201. Fritzsch, H., Minkowski, P. 1975. Ann. Phys. 93:193, and 1976. Nuc1. Phys. B103:61.

202. Gursey, F., Serdaroglu, M. 1978. Yale Report.

203. Ramond, P. 1976. Nucl. Phys, B110:214.

204. Reines, F., Crouch, M.F, 1974. Phys. Rev. Lett. 32:493.

205. Gell-Mann, M., Ramond, P., Slansky, R. 1977. Los Alamos Report LA-UR-77-2059.

206. For a recent discussion along with some speculation and references to other work, see: Weinberg, S. 1977. Harvard Report HUTP-77/A057.

207. GeI'fand, Y., Likhtman, E.P. 1971. 2h. E. T. F. Pif. Red. 13:452 (in English translation 13:323); Wess, J., Zumino, B. 1974. Phys. Lett. $49 \mathrm{~B}: 54$.

208. Freedman, D., van Nieuwenhuizen, P., Ferrara, S. 1976. Phys. Rev. D13:3214; Deser, S., Zumino, B. 1976. Phys. Lett. 62B:335.

209. Grisaru, M., van Nieuwenhuizen, P., Vermaseren, J. 1976. Phys. Rev. Lett. $37: 1662$. 

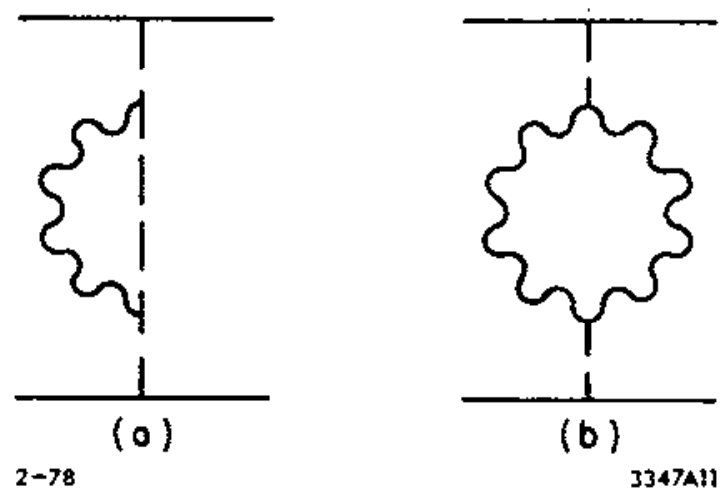

Fig. 1

Contributions to coupling constant renormalization for the Yang-Milis theory in Coulomb gauge. 


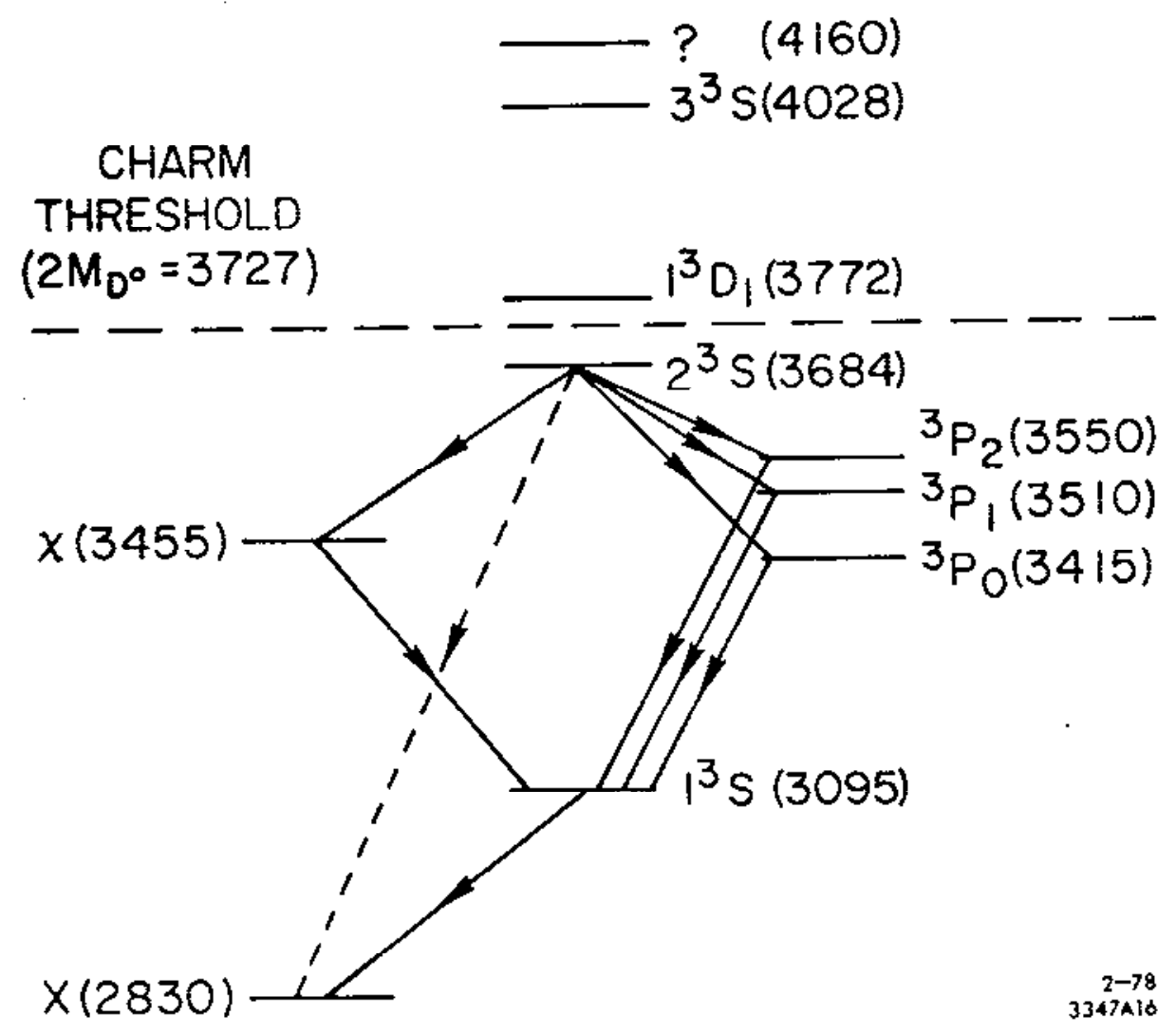

Fig. 2

The observed charmonium levels with the notation $n^{2 S+1} L_{J}$ and masses in MeV. The correct identification for $X(2830)$ and $x(3455)$ is not known. Observed radiative transitions are shown as solid directed 1ines. The dotted line represents an $M$ transition which has not yet been observed. Splittings are approximately to scale. 


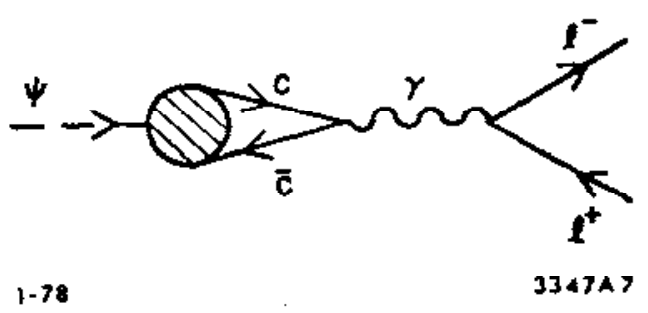

Fig. 3

The electromagnetic decay of the $\psi\left(1^{3} \mathrm{~S}_{1}\right)$. 


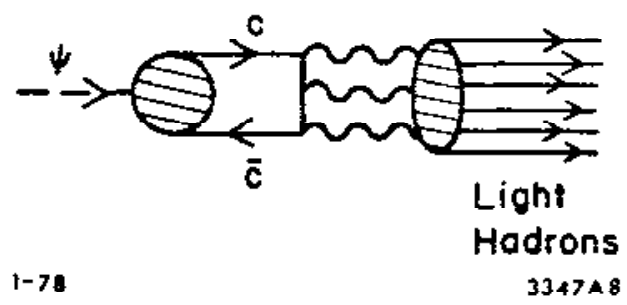

Fig. 4

The hadronic decay of the $\psi\left(1^{3} \mathrm{~s}_{1}\right)$, assuming the minimal gluon mechanism. 


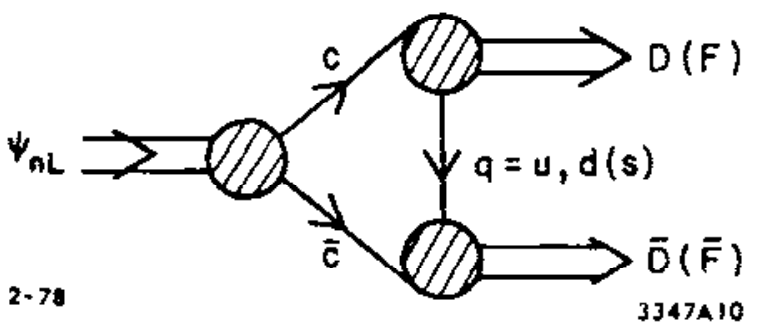

Fig. 5

The transition amplitude (Eq. 3.53)

for $\psi_{\mathrm{nL}} \rightarrow \mathrm{D} \overline{\mathrm{D}}$ or $\mathrm{F} \overline{\mathrm{F}}$. 


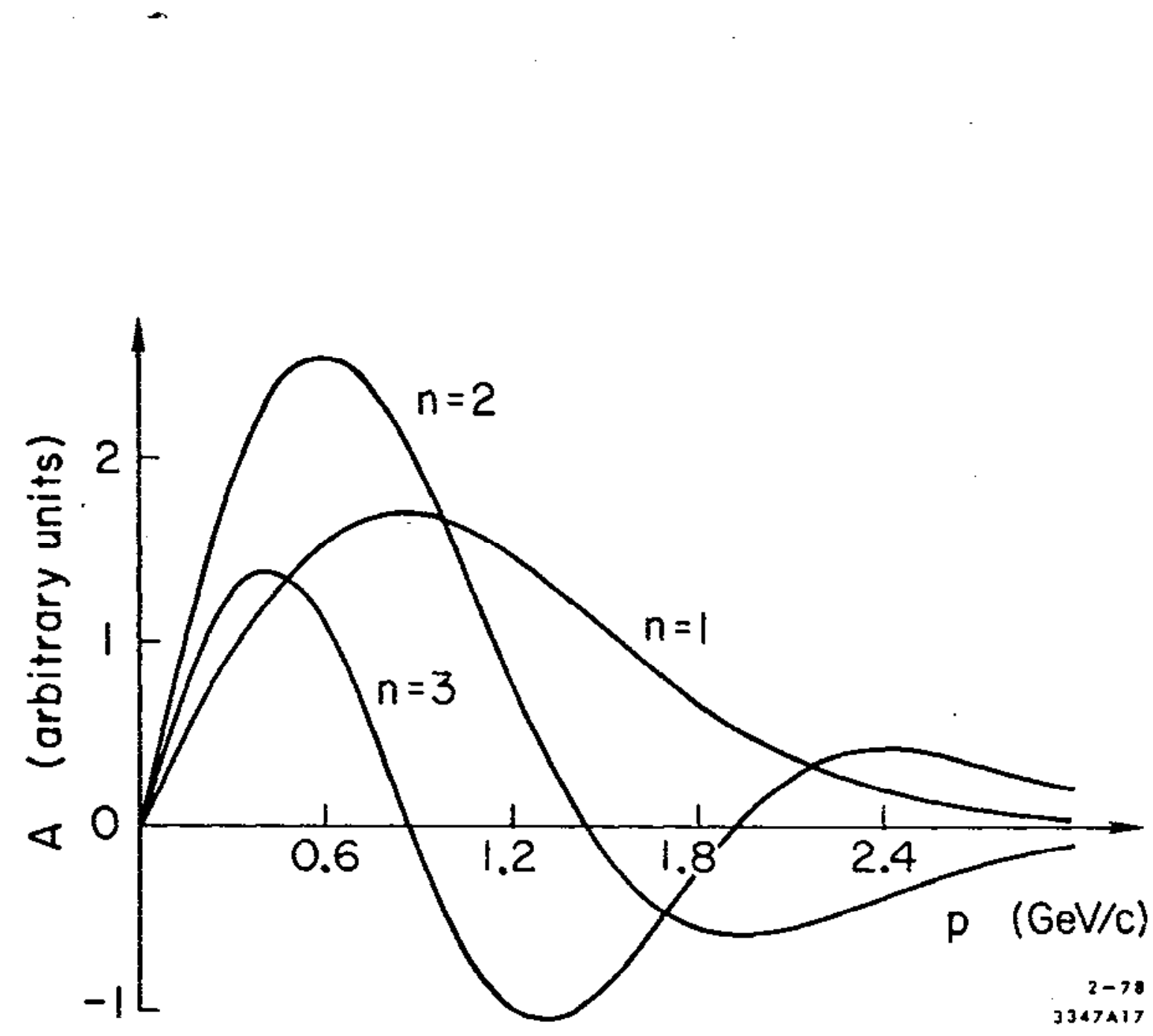

$\mathrm{Fi} 6$

The P-wave form factor in Eq. (3.53) for $\psi_{n 0}+\overline{D D}(n=1,2,3)$ as a function of the momentum of the outgoing charmed meson, $p=$ $\left(W^{2}-4 M_{D}^{2}\right)^{1 / 2} / 2$ (from Ref. 68). See Table 7 for parameters used in this calculation. 


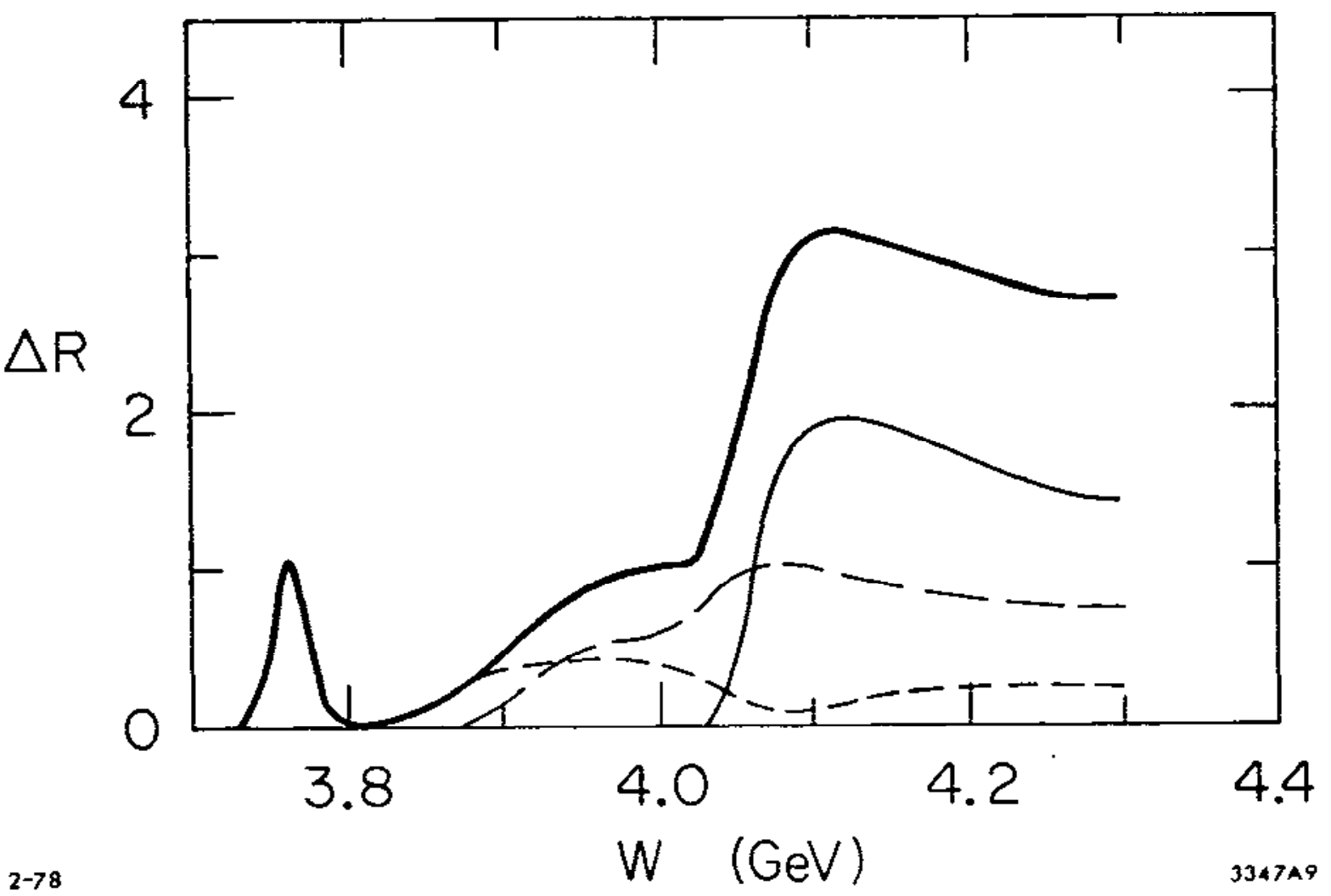

Fig. 7

The charm contribution to $R$ as computed in the coupled-channel model (Ref. 94). The heavy solid curve is the sum of the contributions from $\overrightarrow{D D}$ (short-dashed curve), $\overrightarrow{D^{*}}+D^{*} \bar{D}$ (long-dashed), and $D^{*} \vec{D}^{*}$ (1ight solid); F-meson production makes a negligible contribution. The thresholds used are given in the text; other parameters are very similar to those in Table 7. 


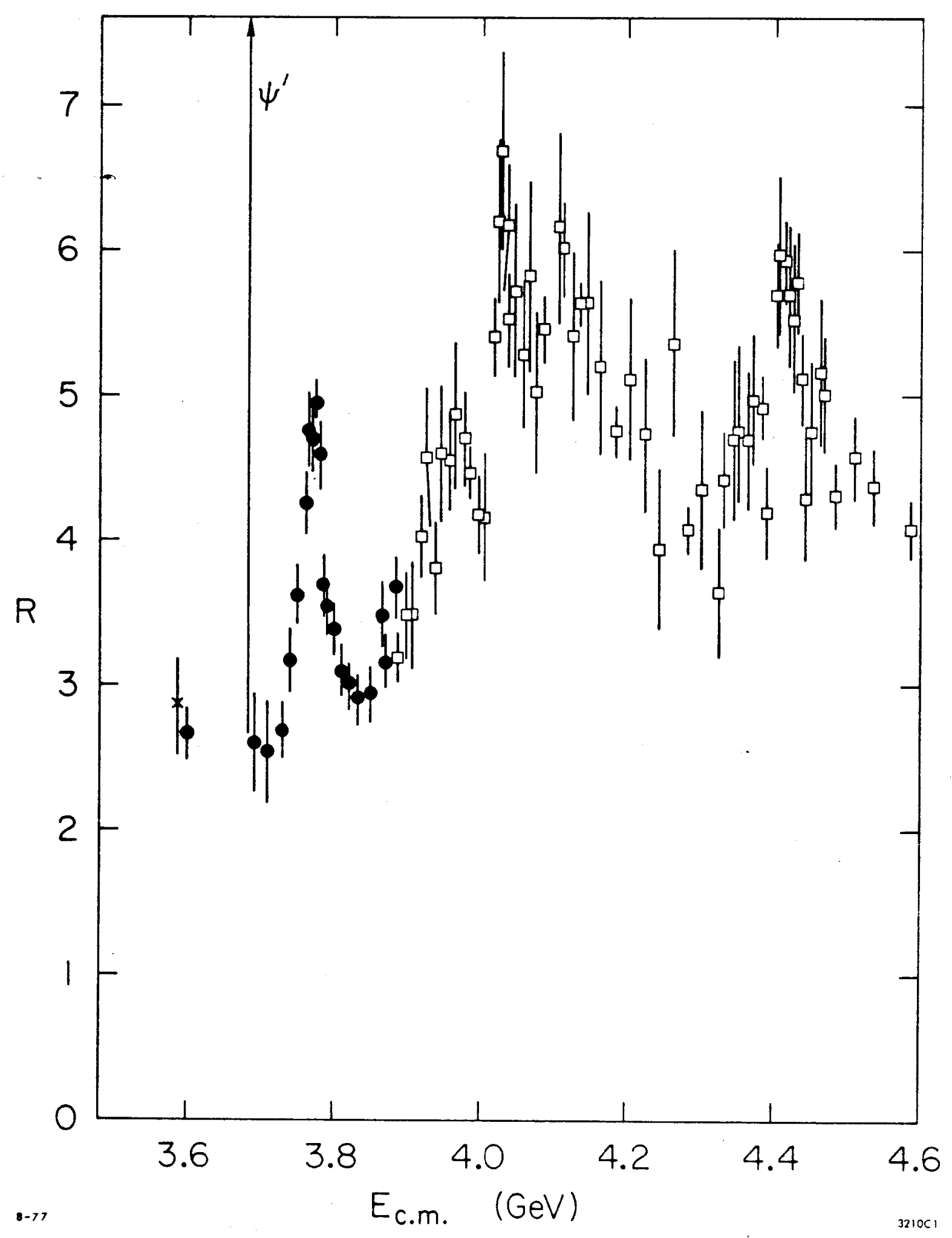

Fig. 8

$R$ in the charm threshold region as measured by the SLAC-LBL collaboration (31) at SLAC. 


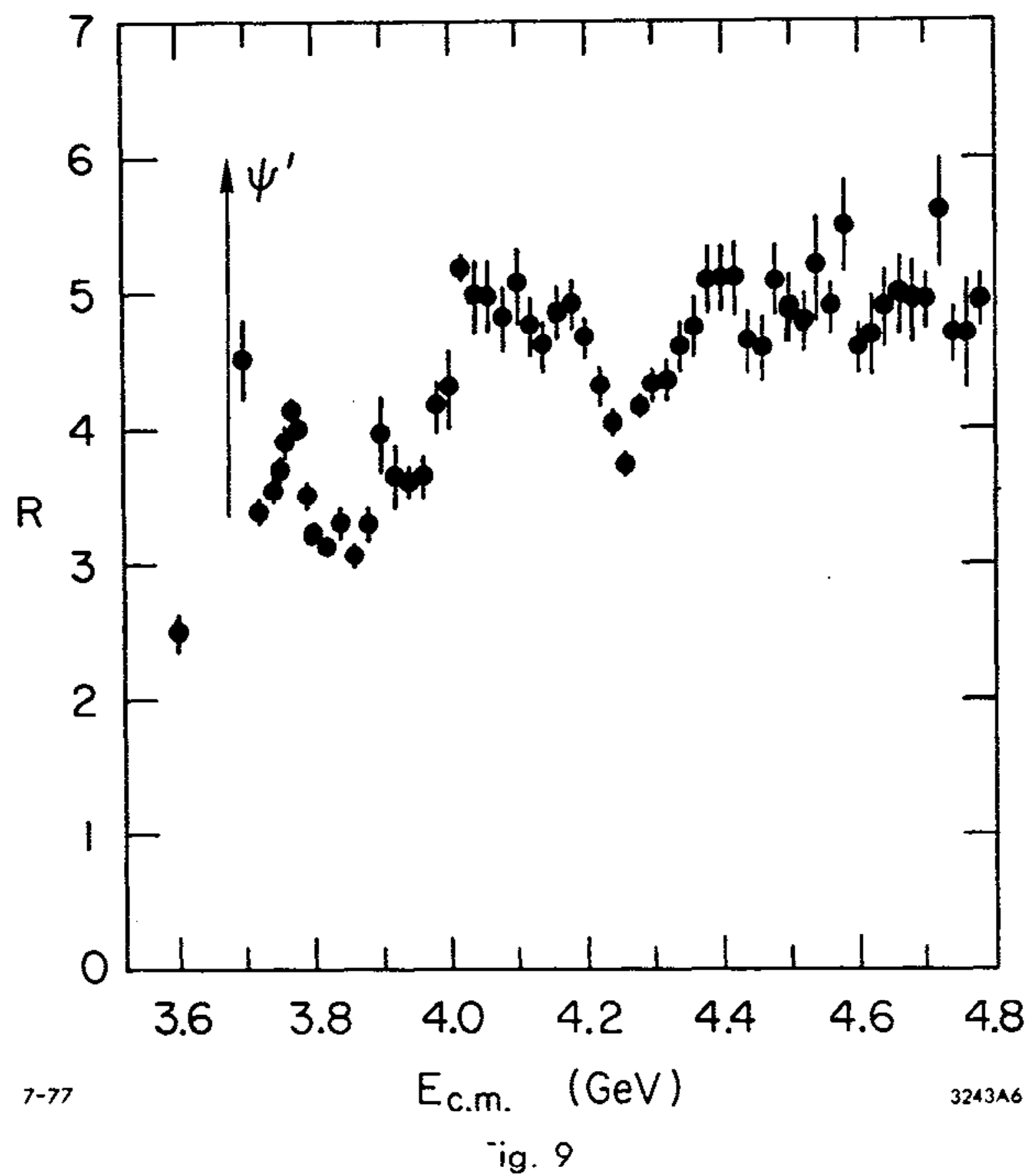

$R$ as measured by the DELCO collaboration (32) at SLAC. 


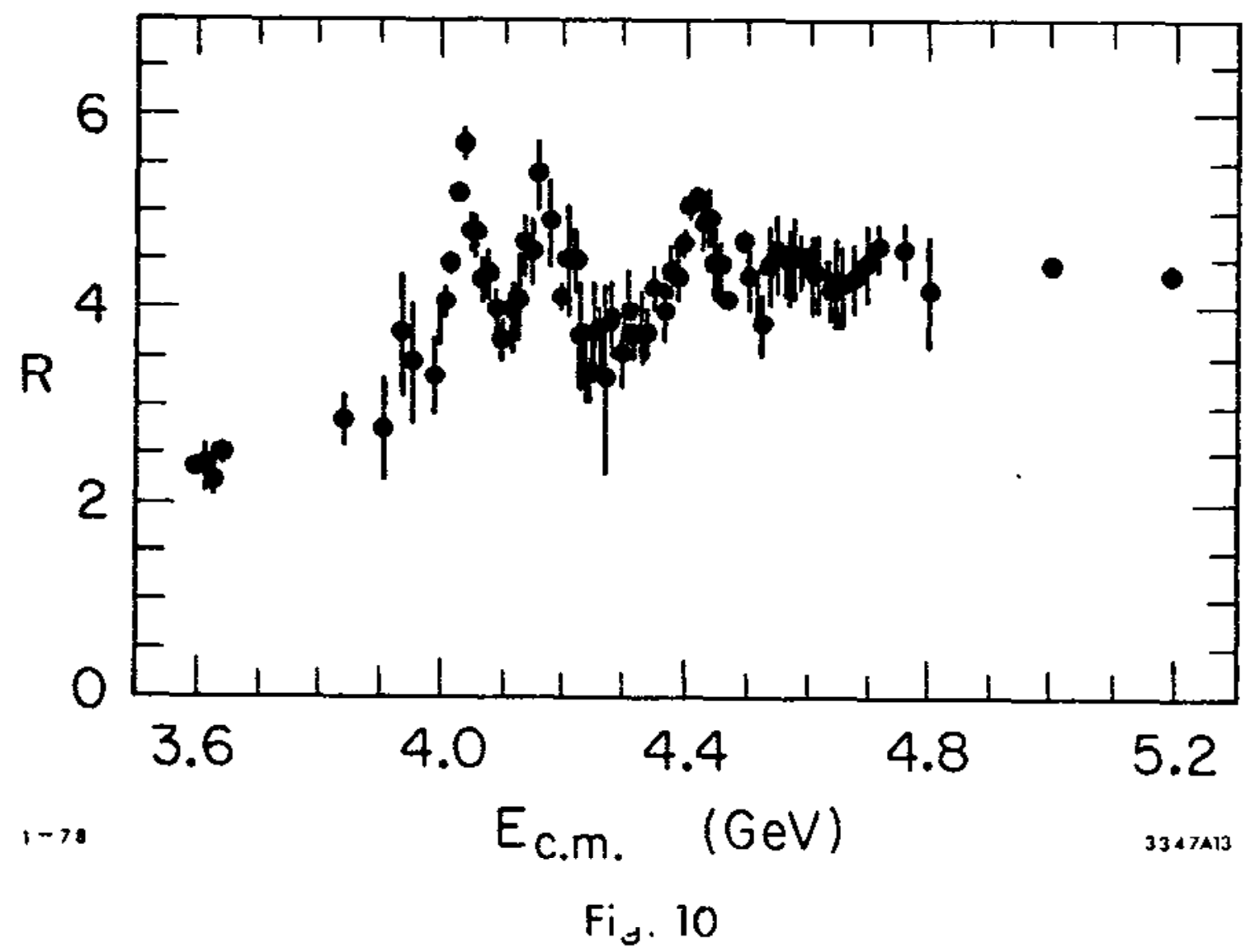

$R$ as measured by the DASP collaboration (33) at DESY. 


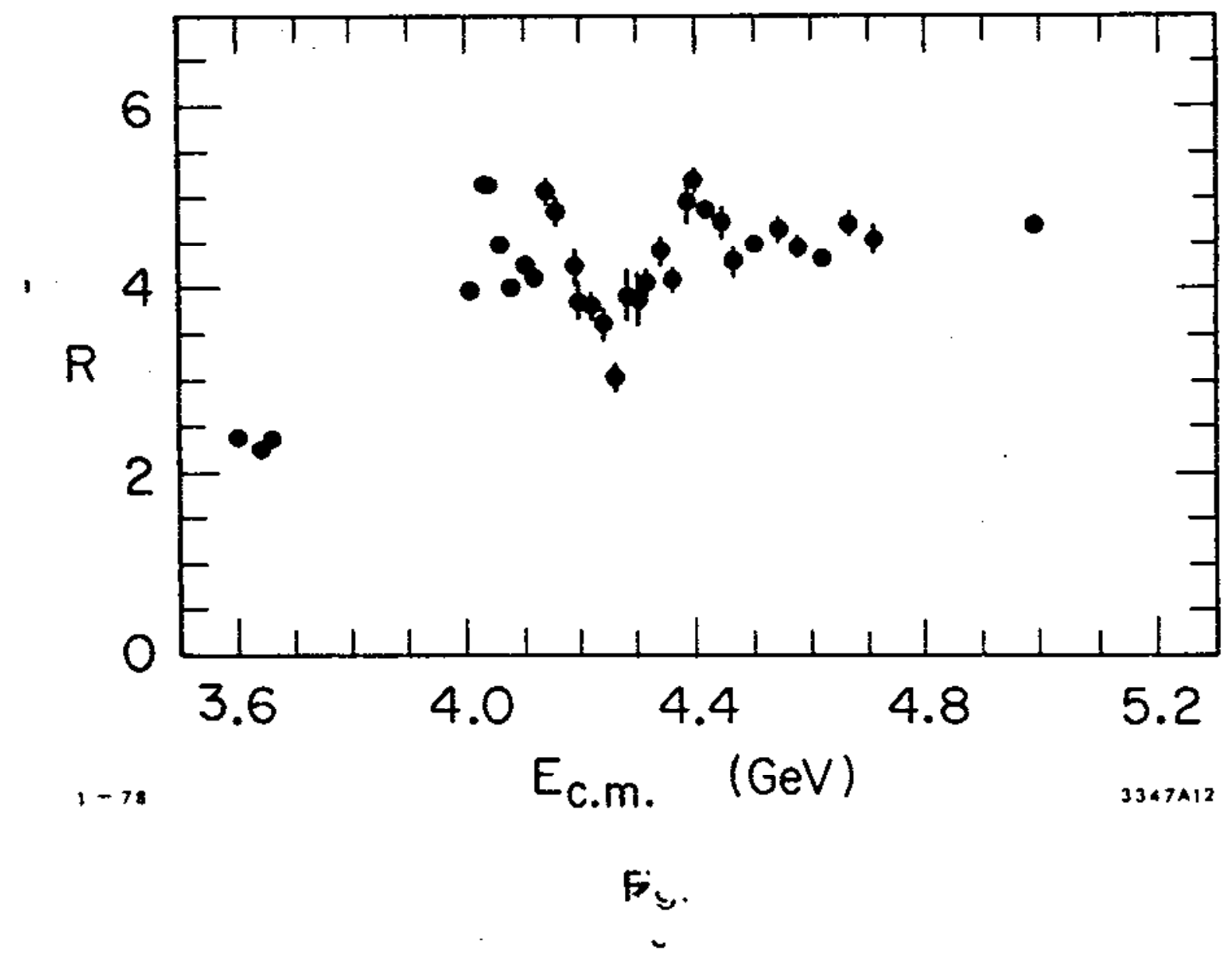

$R$ as measured by the PLUTO collaboration (34) at DESY. 


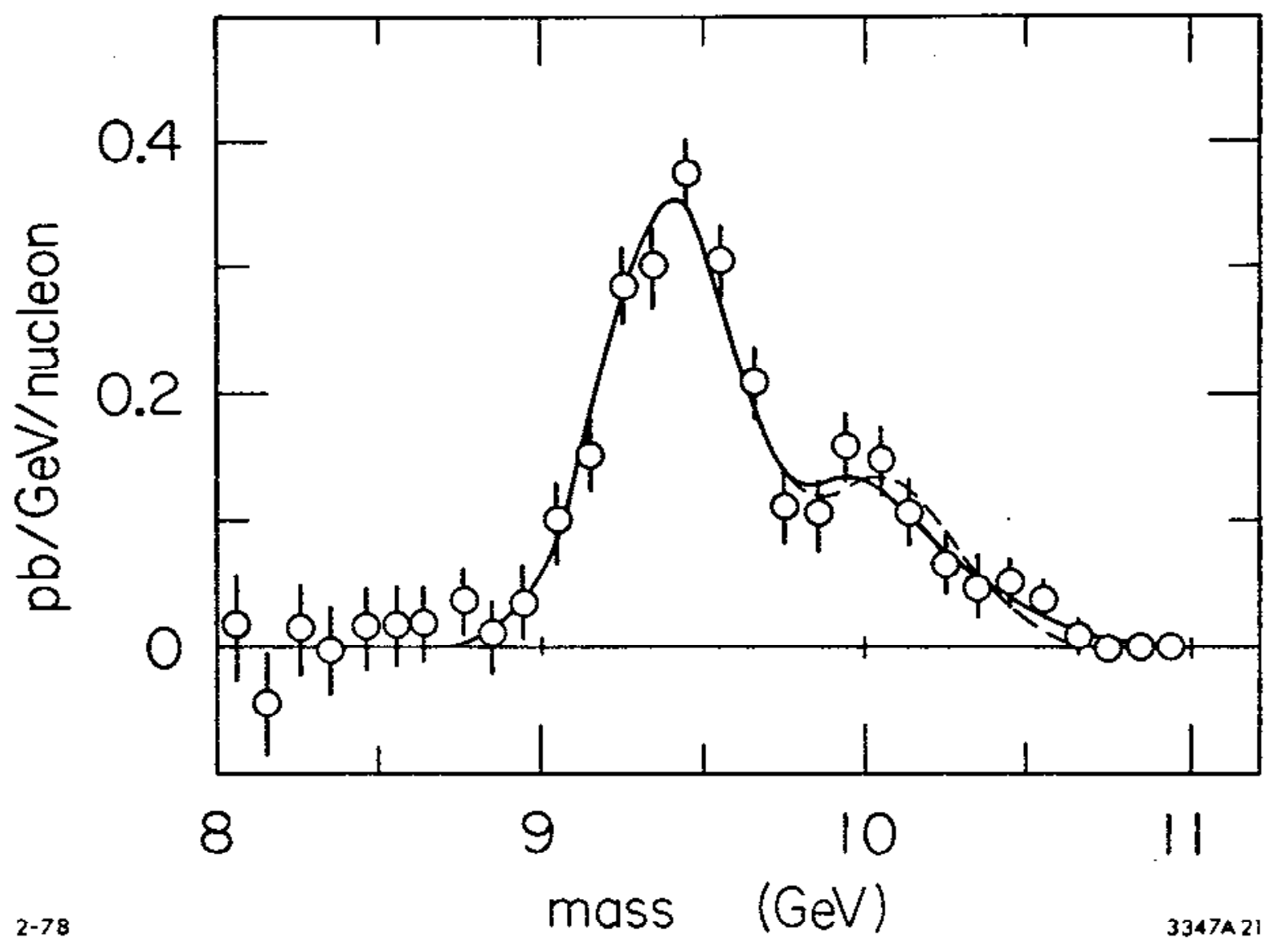

Fig. 12

The $\mu^{+} \mu^{-}$invariant mass spectrum (background subtracted) in the $T^{-}$ region (23). The solid curve is a fit to two zero-width resonances (smeared by resolution); the dashed curve is a fit to three resonances. 


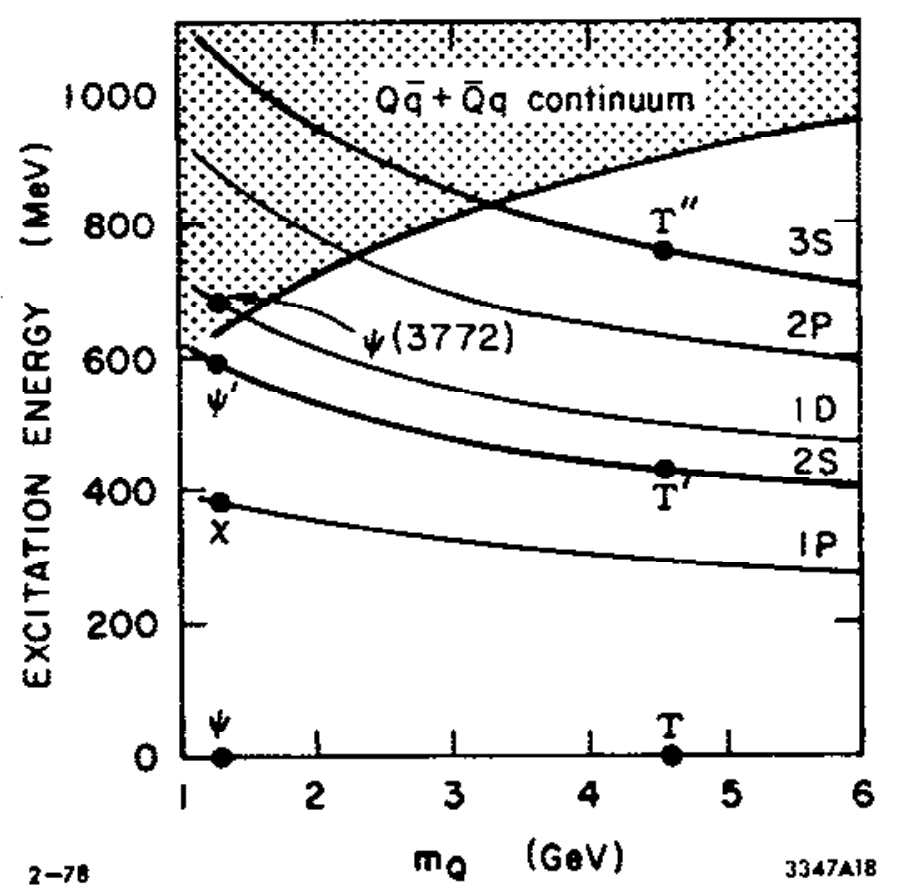

Fig. ${ }^{13}$.

Predicted excitation spectrum of $\bar{Q} \bar{Q}$ Ievels and the threshold for OzI-allowed decays, as a function of the heavy quark mass, $\mathrm{m}_{Q}$, in the Iinear + Coulomb potential model (104) with $\mathbb{m}_{c}=1.37 \mathrm{GeV}$. 


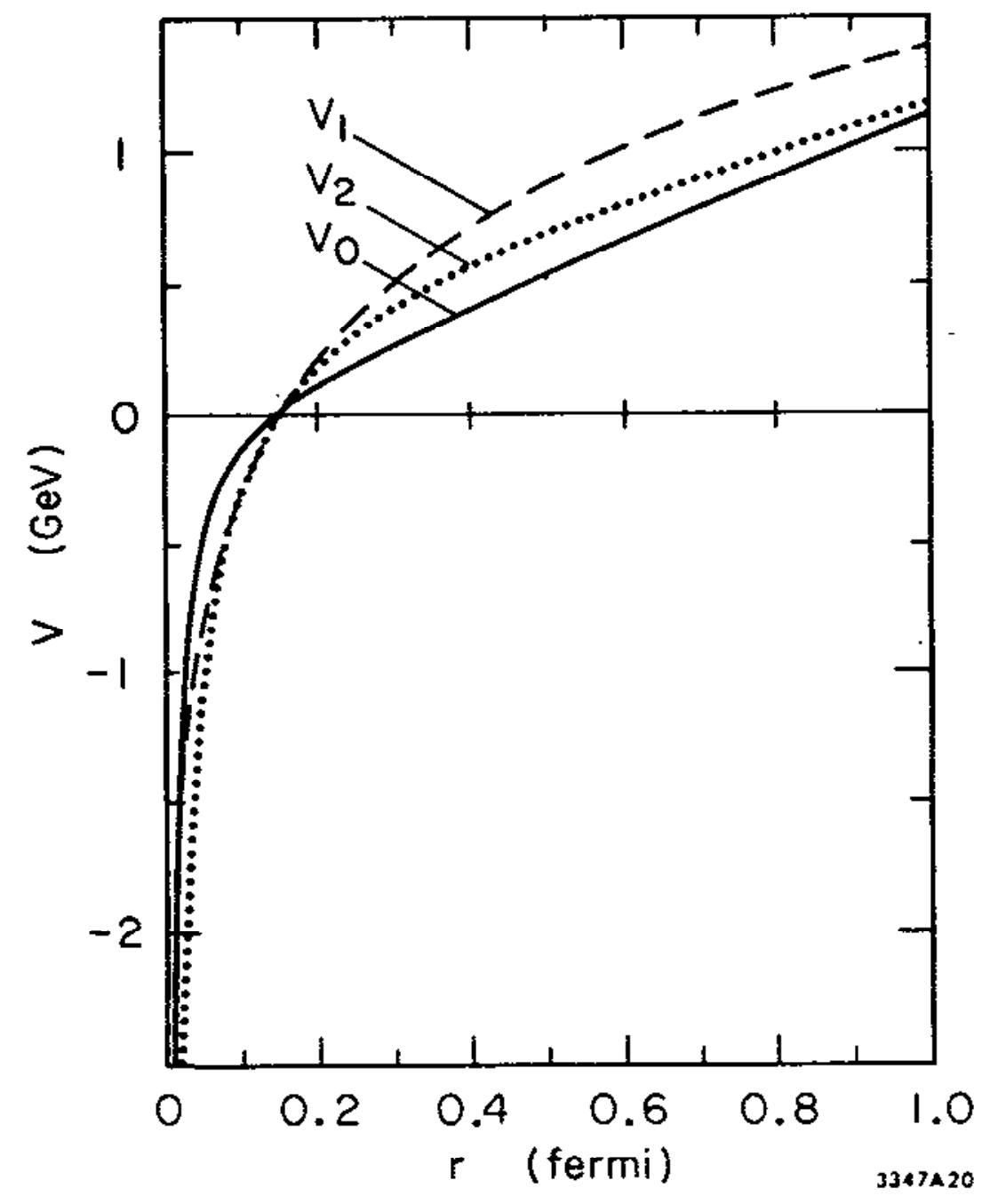

$2-78$

Fig. 4

The $\bar{Q}$ potentials $V_{0}$ (Ref. 68), $v_{1}$ (Ref. 102), and $V_{2}$ (Ref. 86).

The zeroes of energy have been chosen to make them cross at the same value of $r$. 


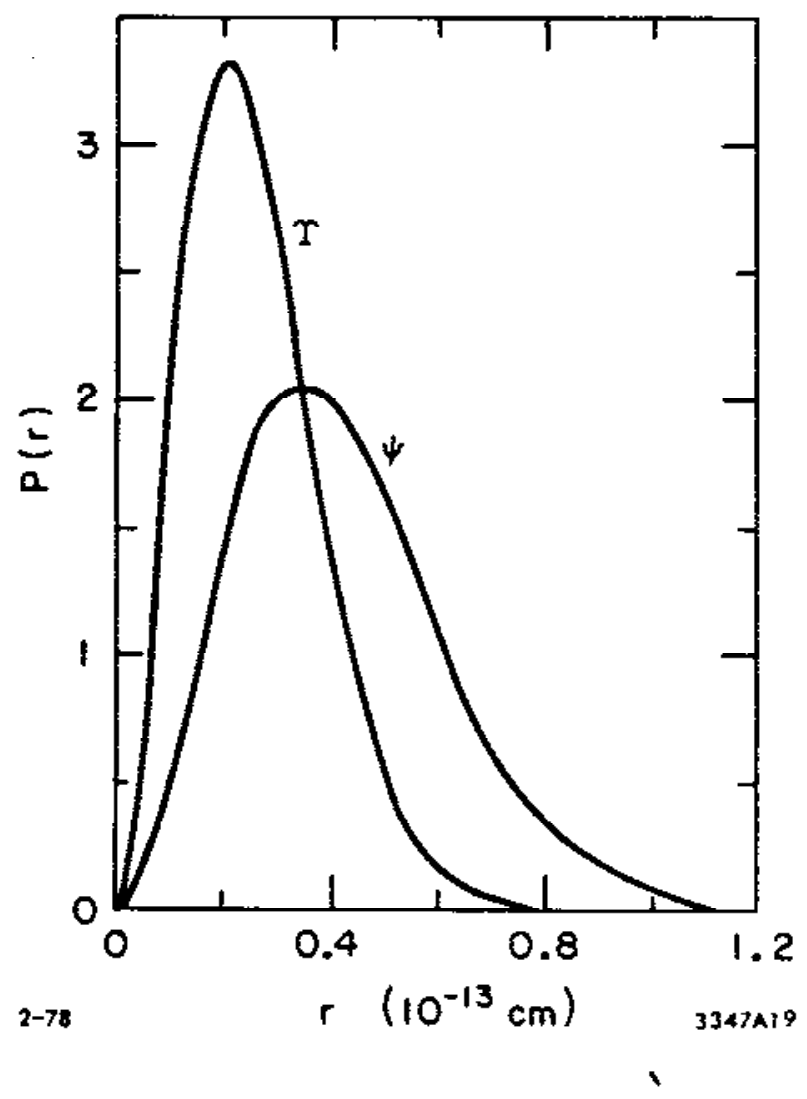

$\mathrm{Fg} \cdot 5$

Radial probability for the $\psi$ and $T$ ground states, computed from $v_{0}$ (Ref. 68), as a function of the $Q \bar{Q}$ separation $r$. 


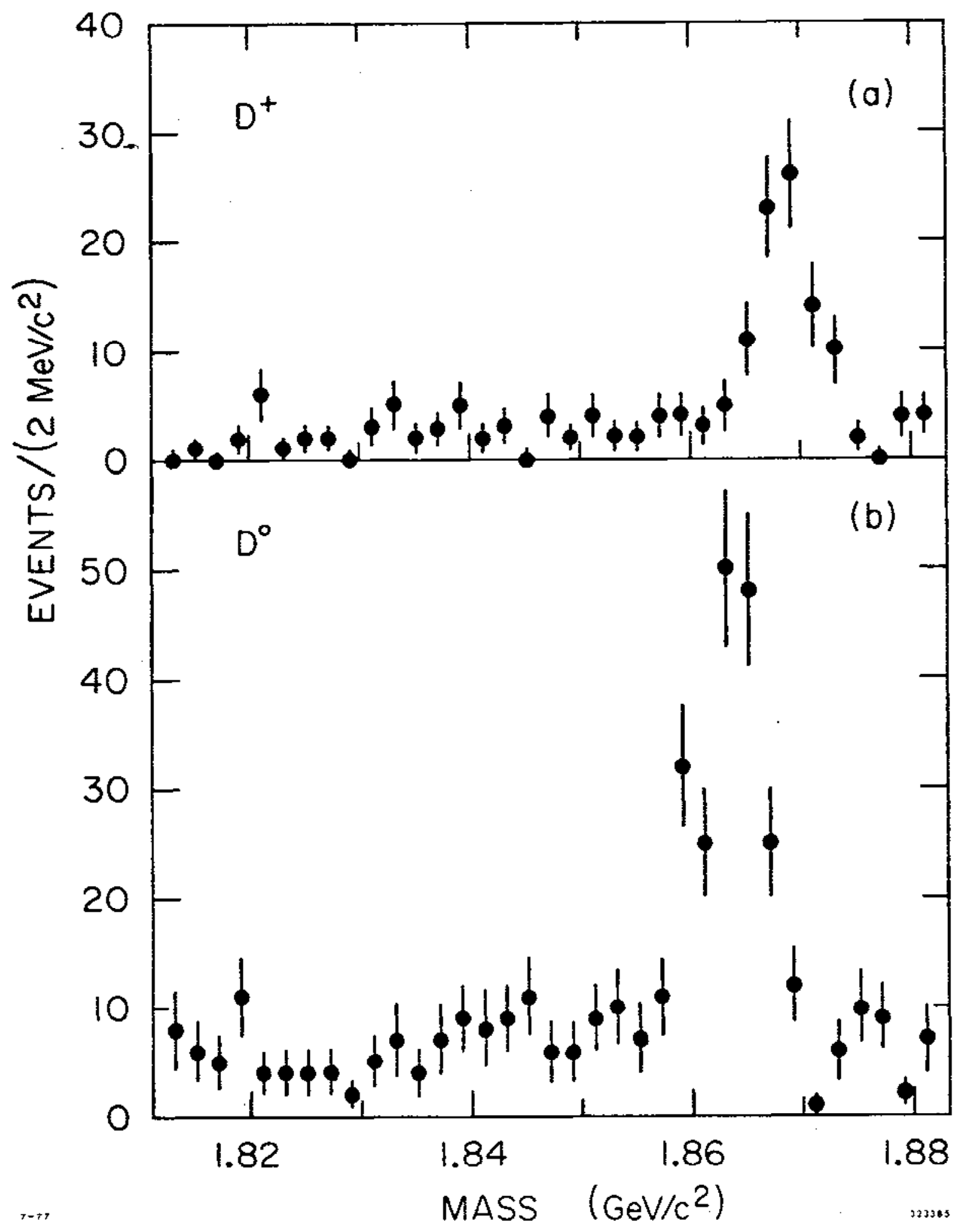

Fig 16

Invariant mass spectra for the sum of all observed (a) $\mathrm{D}^{+}$and (b) $D^{\circ}$ decay modes yielding all-charged-particle final states (108). 


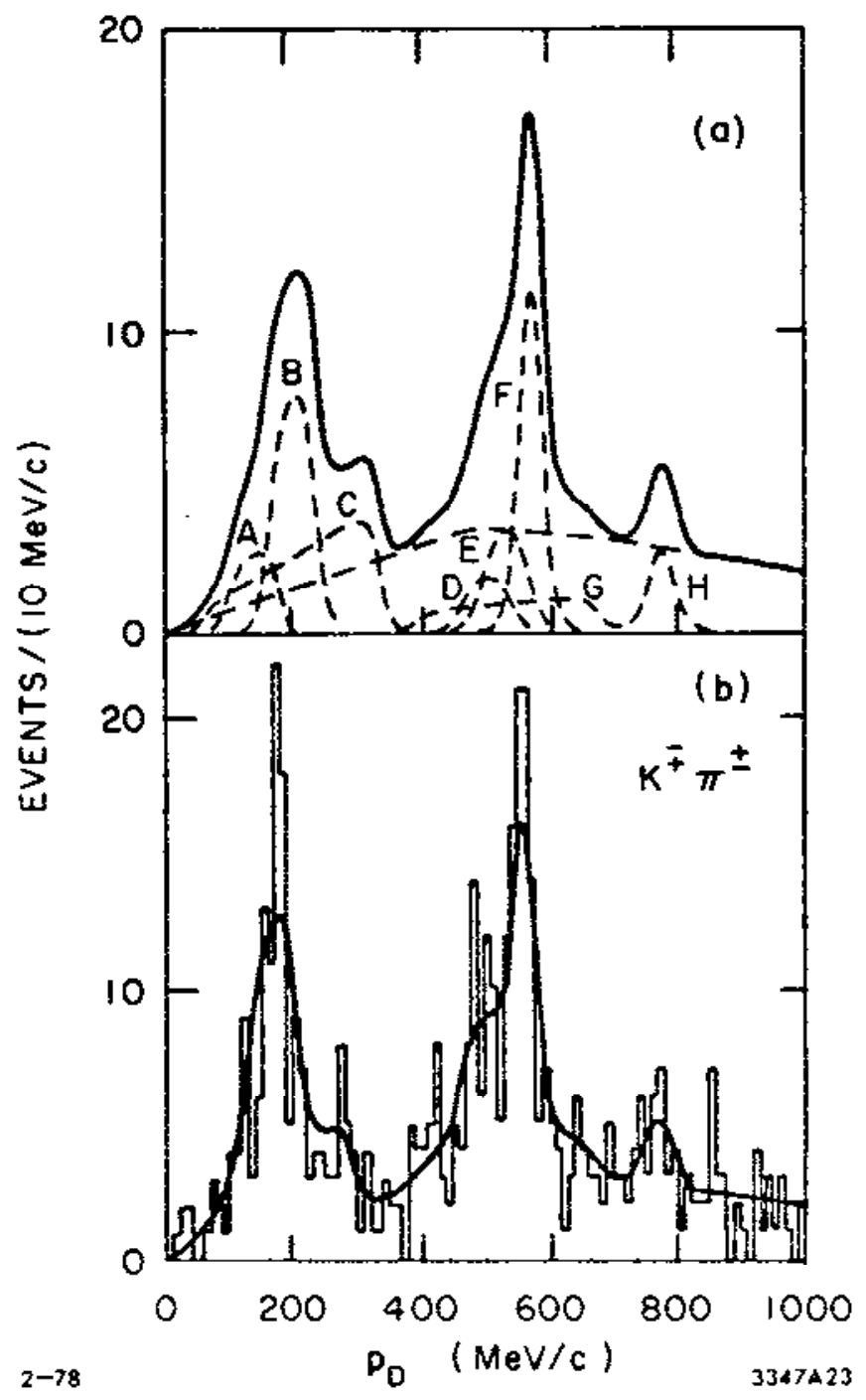

rig. $1^{7}$

The $\mathrm{D}^{\circ}$ momentum spectrum at $4.028 \mathrm{GeV}$, for $\mathrm{D}^{\circ} \rightarrow \mathrm{K}^{{ }^{+}} \pi^{ \pm}$(from Ref. 99) The solid curves represent an isospin-constrained fit to the data. (a) shows the various contributions to the fit in (b). Curves $A$, $B, C$ are from $e^{+} e^{-} \rightarrow D^{* D^{*}}$ with (A) $D^{*+} \rightarrow D^{\circ} \pi^{+}$, (B) $D^{*_{O}} \rightarrow D^{\circ} \pi^{\circ}$, and (C) $D^{* 0}+D^{\circ} \gamma, D, E, F, G$ are from $D^{*} D+\vec{D}^{*} D$ production with (D) $\mathrm{D}^{{ }^{+}} \rightarrow \mathrm{D}^{\circ} \pi^{+},(E) \mathrm{D}^{*_{0}} \rightarrow \mathrm{D}^{\circ} \pi^{\circ}$, (F) direct $\mathrm{D}^{\circ}$, and (G) $\mathrm{D}^{*_{0}} \rightarrow \mathrm{D}^{\circ} \gamma$. Curve $H$ is the contribution from $D^{\circ} \bar{D}^{\circ}$ production. 


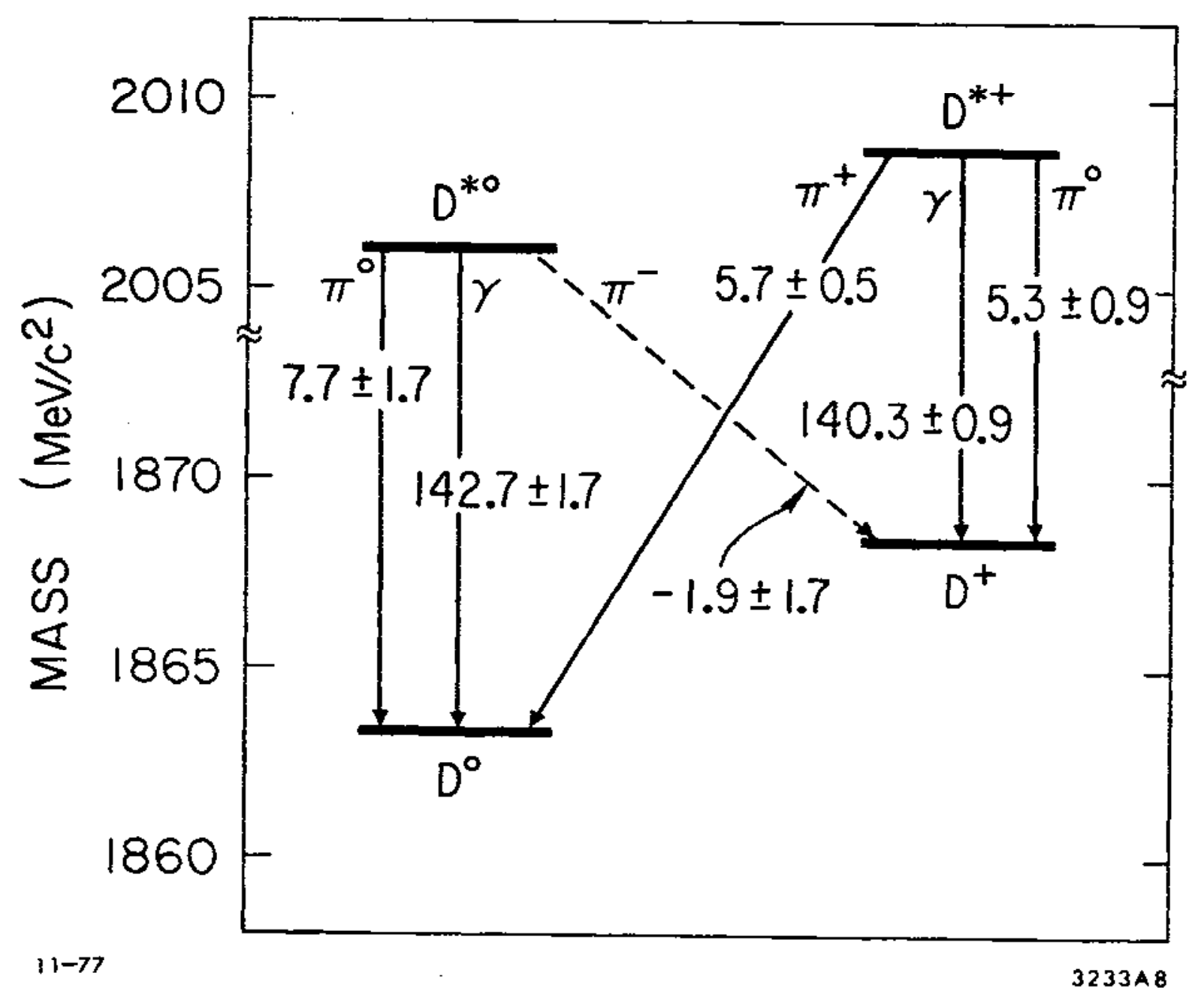

Fig. 18

Q-values for $D^{*} \rightarrow$ D transitions. 


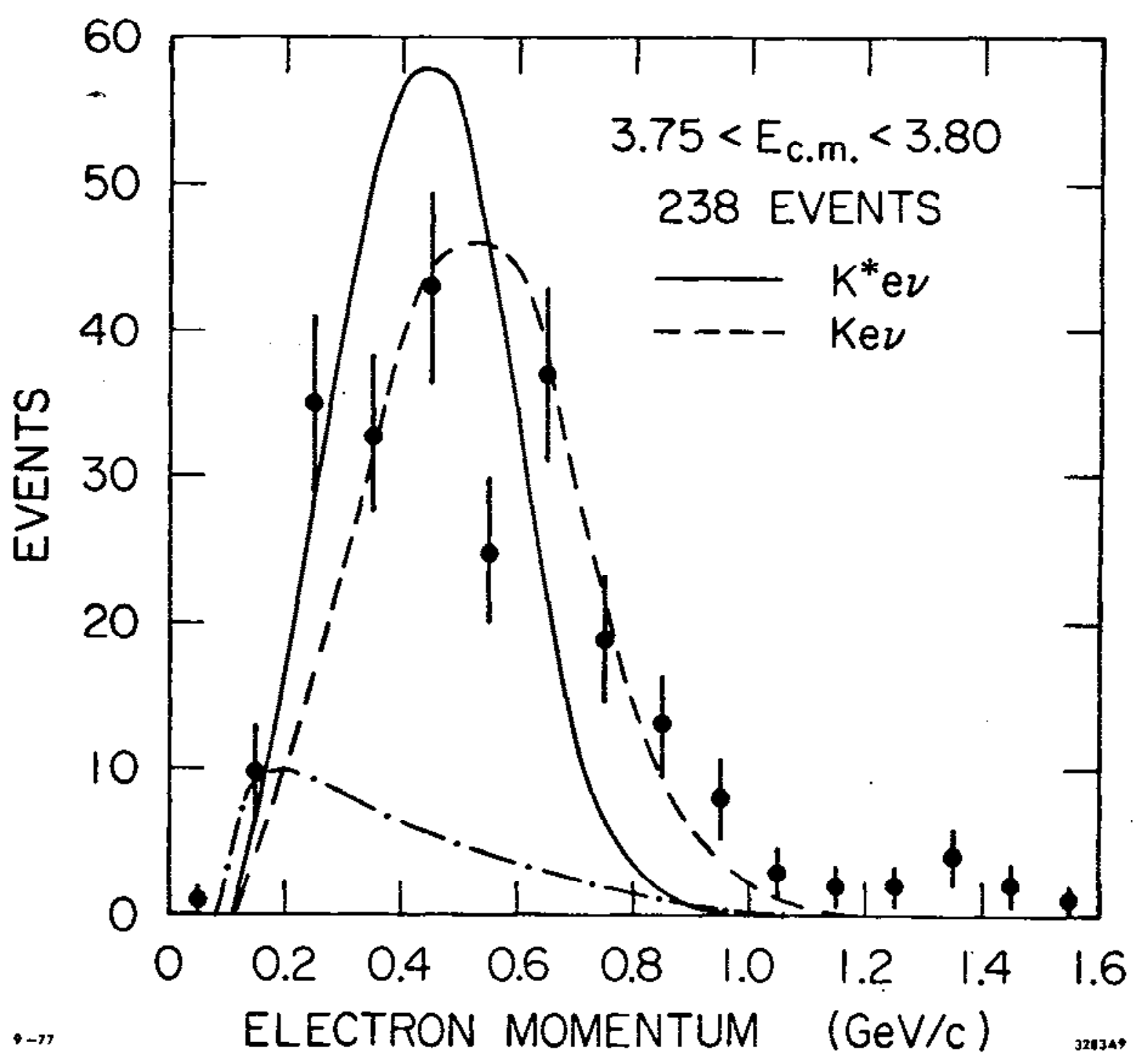

Fig. 19

The momentum spectrum of inclusive electrons in multi-particle decays of the $\psi "$ as measured by the DELCO collaboration (32) at SLAC. Solid and dashed curves are theoretical spectra expected for $D \rightarrow K^{\star}$ ev and $D \rightarrow$ Kev. The dot-dashed curve indicates the estimated background remaining in the data. 


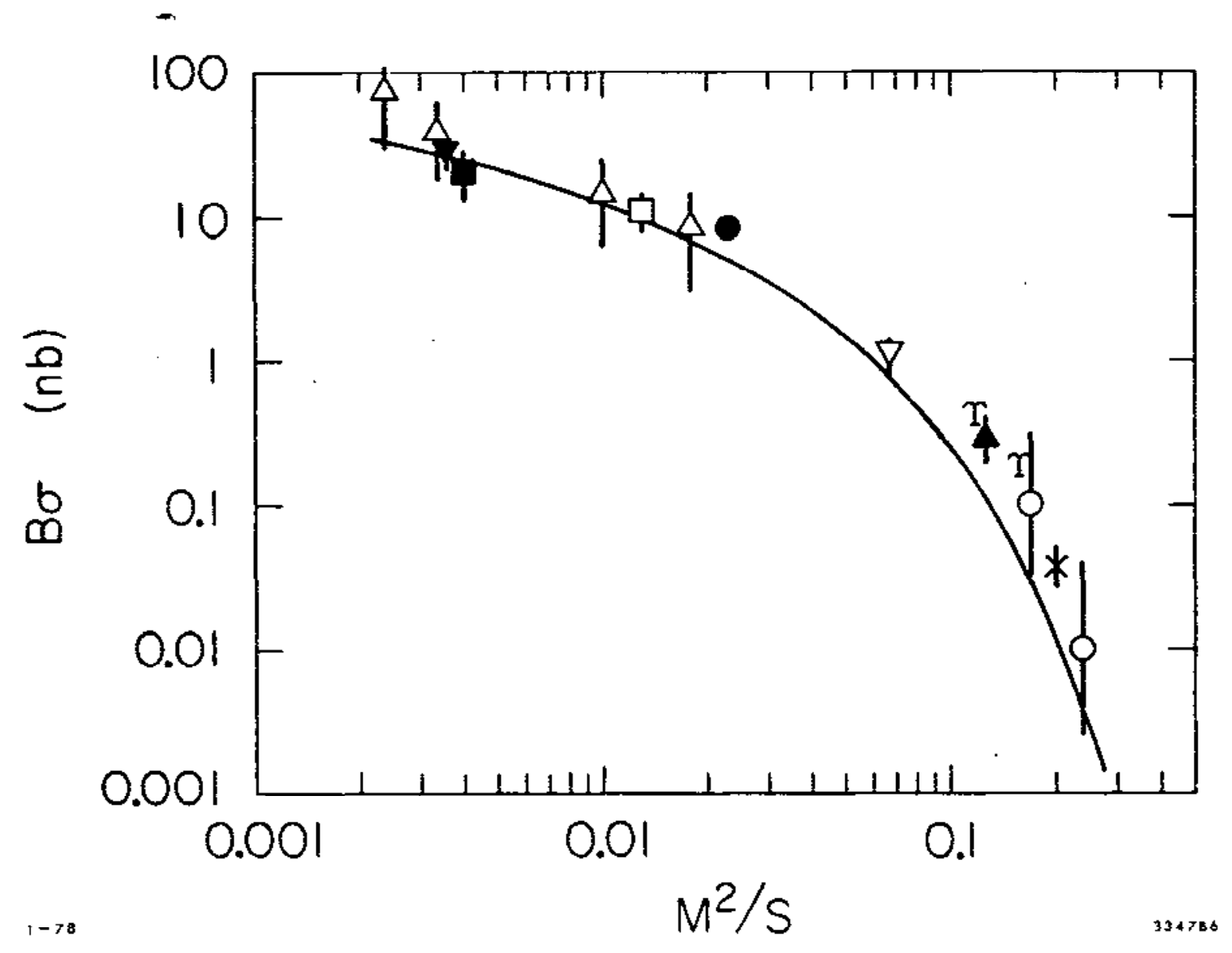

Fig. 20

The cross-section for $\psi$ production in po scattering times the branchIng ratio to muons as a function of $\tau$ (where $\tau \equiv \mathrm{m}_{\psi}^{2} / \mathrm{s}$ ). The data 1s from Ref. 153. The curve is a theoretical prediction (156) of Carlson and Suaya. The two symbols $T$ are the data for $T$ production adjusted according to Eq. 6.3 . 


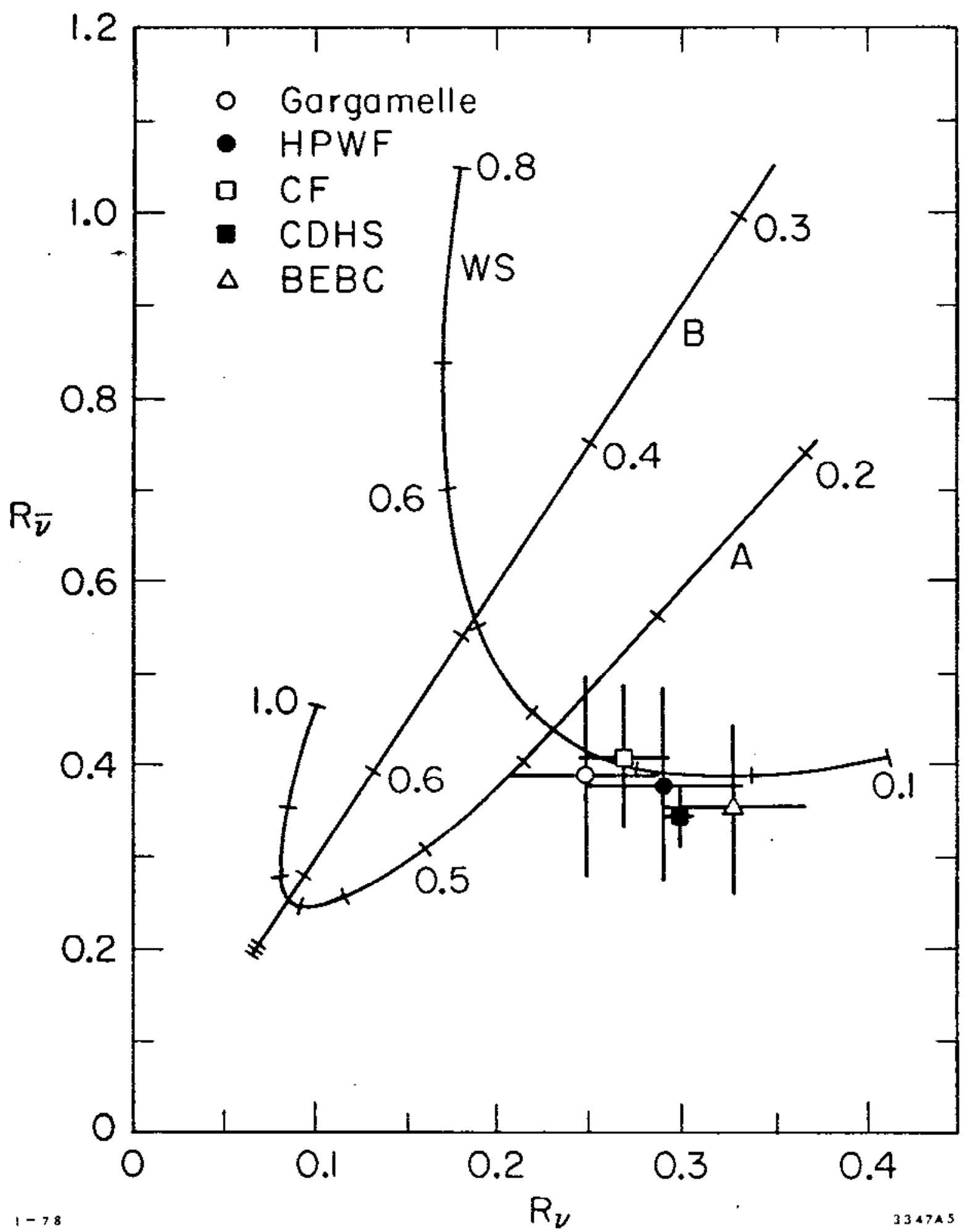

Fig. 21

The ratio $\sigma(\nu N \rightarrow \nu+X) / \sigma(\nu N+\mu+X)$ for antineutrinos versus that ratio for neutrinos. The tenth values of $\sin ^{2} \theta_{W}$ are shown with tick marks on the theoretical curves. The curve labelled A refers to the model with $(u b)_{R}$ : the curve labelled $B$ refers to the model with both $(u b)_{R}$ and $(t d)_{R}$. The data is from Ref. 175 . 


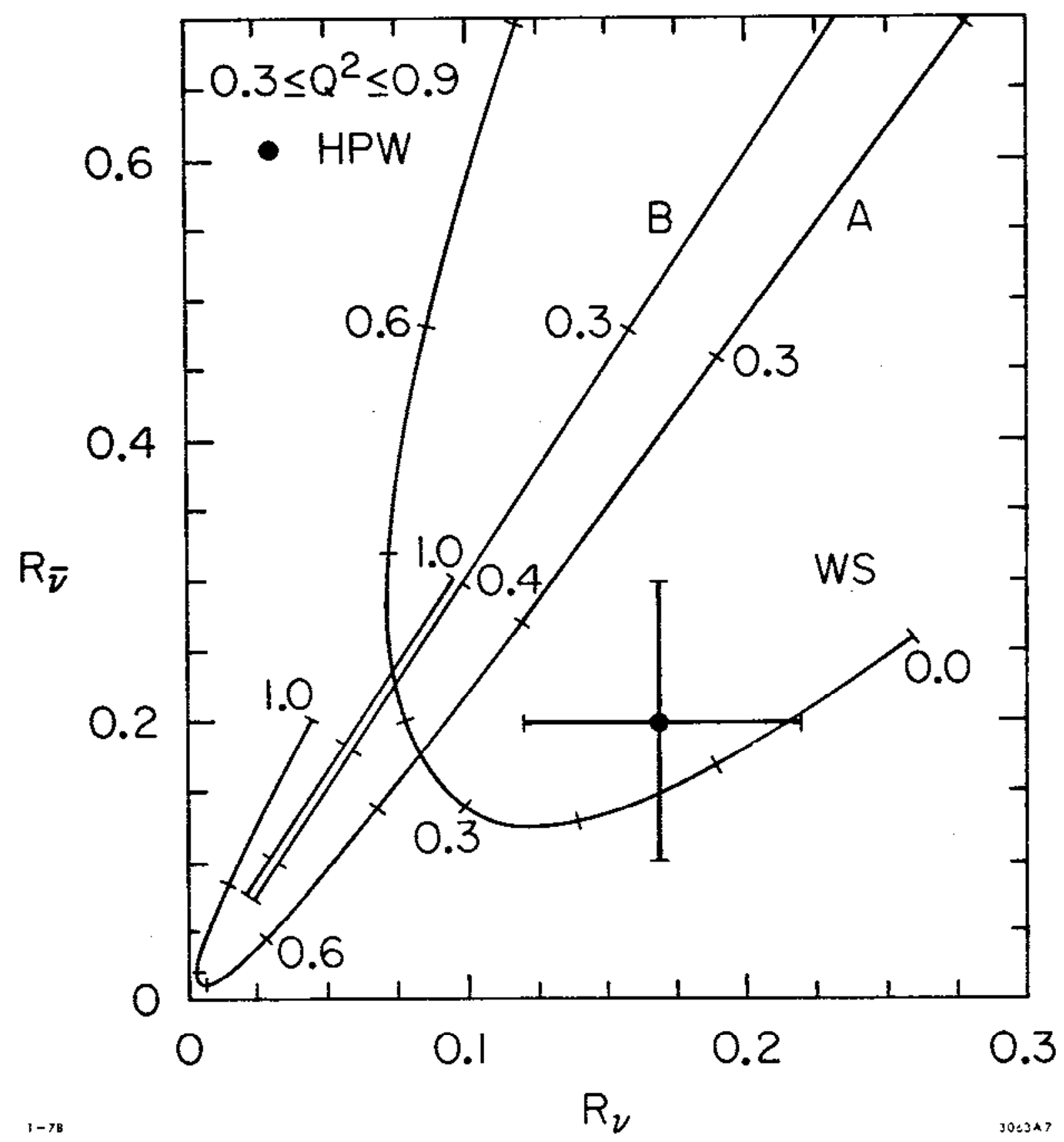

Fig. 22

The ratio $\sigma\left(\bar{v}_{p} \rightarrow \bar{v}_{p}\right) / \sigma\left(\bar{v}_{p} \rightarrow \mu{ }^{+} n\right)$ versus the ratio $\sigma\left(v_{p} \rightarrow v_{p}\right) / \sigma\left(v_{n} \rightarrow \mu^{-} p\right)$. The notation is the same as for Fig. 21. The data shown is from D. Cline et al. (Ref. 176). 

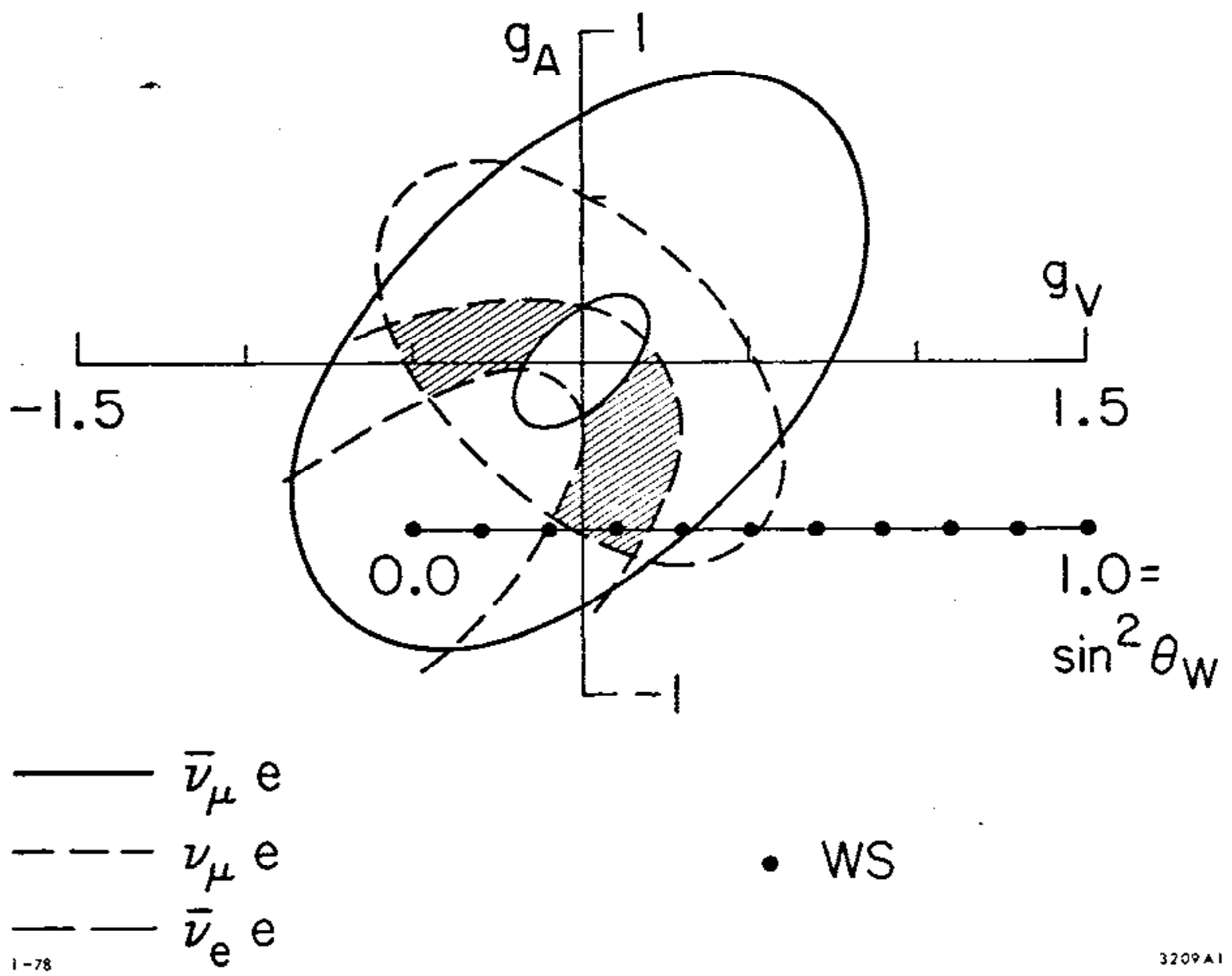

- WS

Fig. 23

The 11mits placed on $g_{A}$ and $g_{V}$ by data for ve scattering. The outer (inner) 1ines indicate $90 \%$ confidence upper (lower) limits. The shaded regions are the overlap or allowed regions for $g_{A}$ and $g_{V}$. The line with dots for tenth values of $\sin ^{2} \theta_{w}$ is the prediction of the wS trodel. The data is from Ref. 178 (the data of Reithler, which was not used, would give somewhat larger values of $g_{A}$ and $g_{V}$ ). 


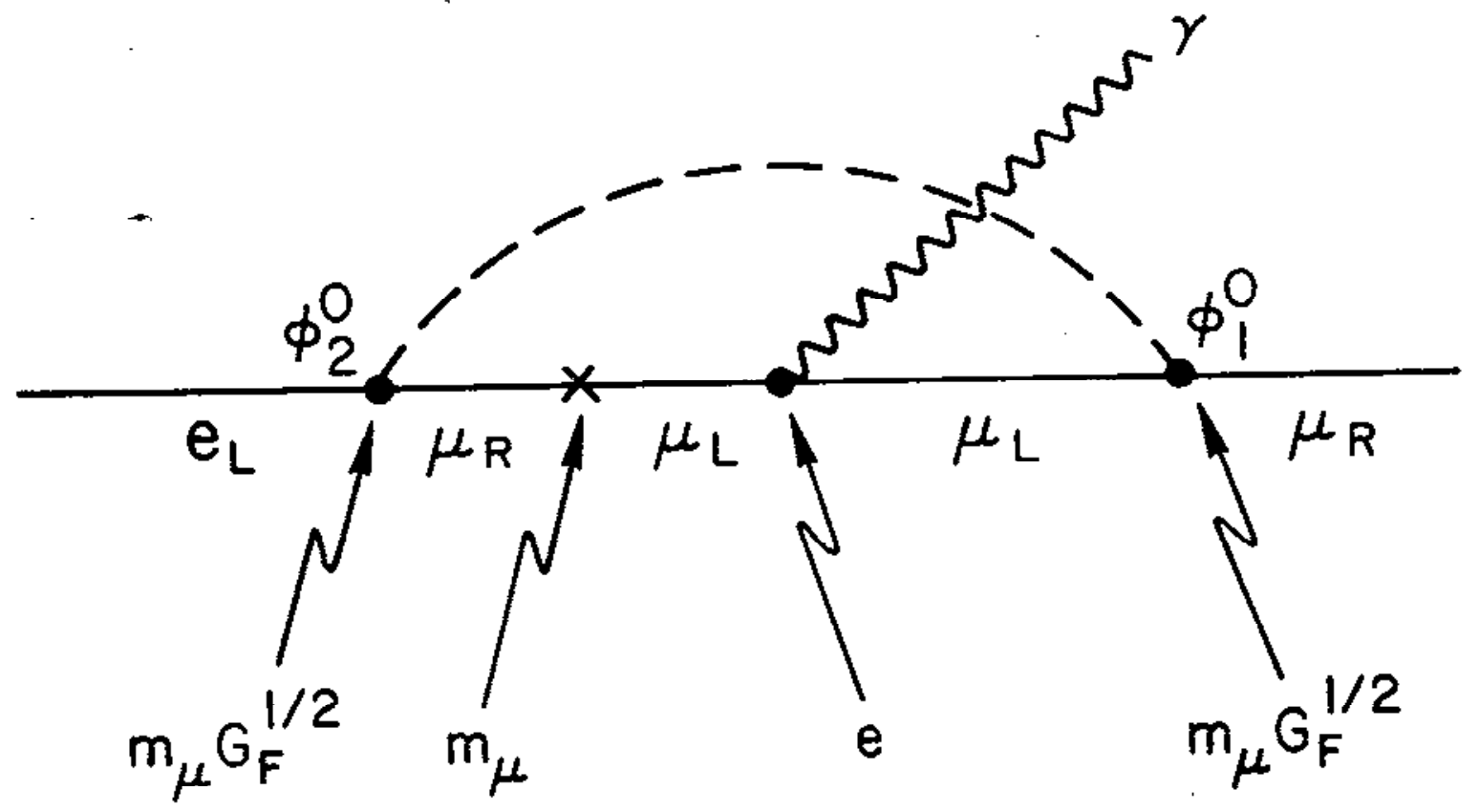

(a)

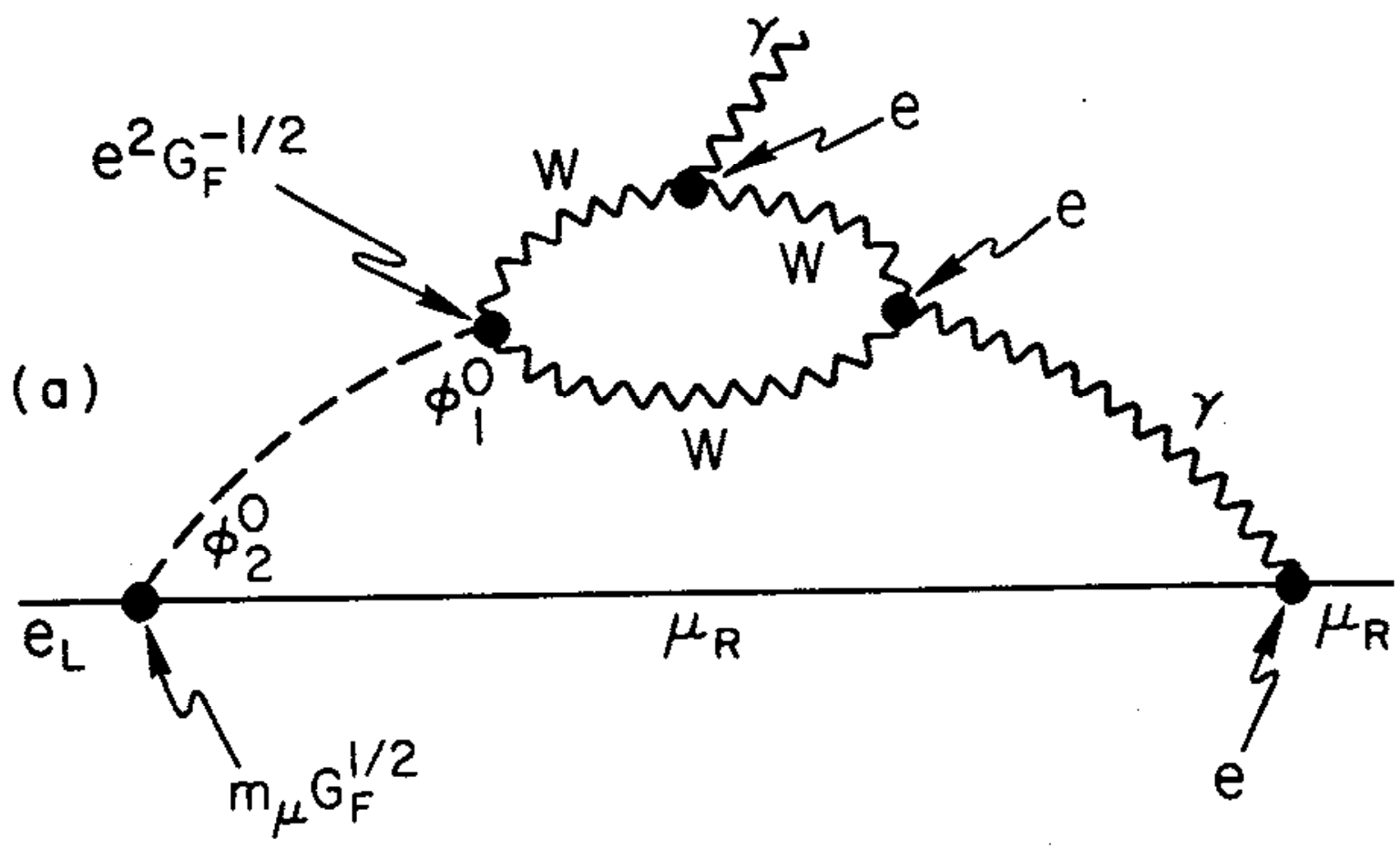

(b)

3092 A 23

Fig. 24

One (a) and two (b) loop diagrams in which virtual Higgs exchange leads to the decay $\mu \rightarrow$ ey. This figure was taken from Ref. 188 . 


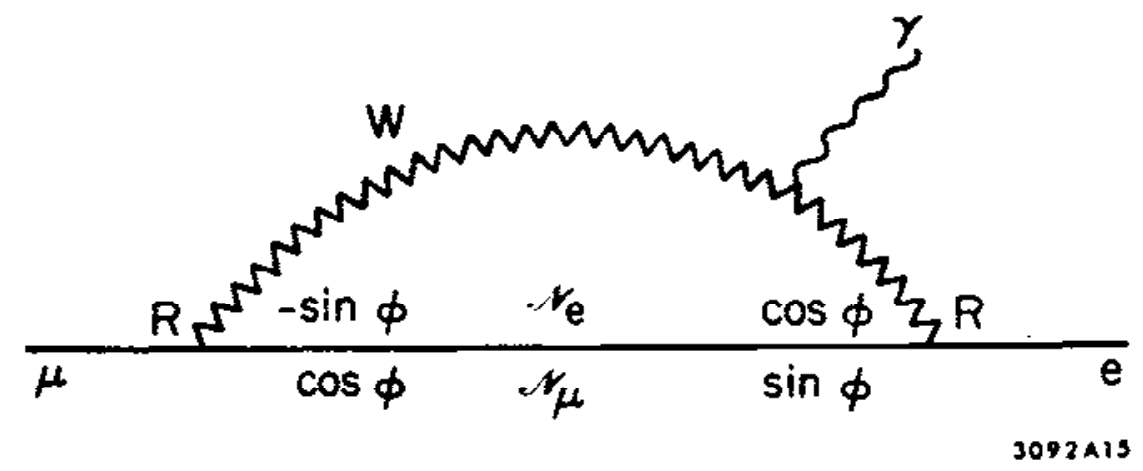

Fig. 25

One of the diagrams in which $\mathscr{N}_{\mathrm{e}}$ and $\mathscr{N}_{\mu}$ exchange leads to the decay $\mu \rightarrow$ er. 